\title{
Roles of the Cpc1 regulator of the cross-pathway control in the Verticillium plant pathogens
}

\author{
Dissertation \\ zur Erlangung des mathematisch-naturwissenschaftlichen Doktorgrades \\ "Doctor rerum naturalium" \\ der Georg-August-Universität Göttingen \\ im Promotionsprogramm GAUSS \\ der Georg-August University School of Science
}

vorgelegt von

Christian Timpner

aus Northeim

Göttingen 2013 
Betreuungsausschuss

Prof. Dr. Gerhard Braus, Abteilung f. molekulare Mikrobiologie und Genetik, Institut für Mikrobiologie und Genetik

Mitglieder der Prüfungskommission

Referent: Prof. Dr. Gerhard Braus, Abteilung f. molekulare Mikrobiologie und Genetik, Institut für Mikrobiologie und Genetik

Korreferentin: Prof. Dr. Andrea Polle, Abteilung Forstbotanik u. Baumphysiologie, Büsgeninstitut

Weitere Mitglieder der Prüfungskommission:

Prof. Dr. Stefanie Pöggeler, Abteilung f. Genetik eukaryotischer Mikroorganismen, Institut für Mikrobiologie und Genetik

Jun.-Prof. Dr. Kai Heimel, Abteilung f. Mikrobielle Zellbiologie, Institut f. Mikrobiologie u. Genetik

PD Dr. Stefan Irniger, Abteilung f. molekulare Mikrobiologie und Genetik, Institut f. Mikrobiologie u. Genetik

PD Dr. Thomas Teichmann, Abteilung f. Zellbiologie der Pflanze, Schwann-Schleiden Zentrum

Tag der mündlichen Prüfung: 23.10.2013 
Die vorliegende Arbeit wurde in der Arbeitsgruppe von Prof. Dr. Gerhard H. Braus in der Abteilung Molekulare Mikrobiologie des Institutes für Mikrobiologie und Genetik der Georg-August-Universität Göttingen angefertigt.

Teile dieser Arbeit wurden veröffentlicht in:

Timpner, C., Braus-Stromeyer, S. A., Tran, V. T. and Braus, G.H. (2013) The Cpc1 regulator of the cross-pathway control of amino acid biosynthesis is required for pathogenicity of the vascular pathogen Verticillium longisporum. Accepted for publication 18.7.2013. 
Für meine Eltern und meine Schwester 


\section{Danksagung:}

Zuerst möchte ich mich bei Herrn Prof. Dr. Gerhard H. Braus für die Möglichkeit, an diesem Thema zu arbeiten und in seiner Abteilung eine Dissertation anzufertigen, bedanken. Bei ihm und Frau Prof. Andrea Polle möchte ich mich für die gute Betreuung sowie viele anregende Gespräche bedanken.

Ebenso bei Frau Dr. Susanna Braus-Stromeyer für ihre gute und umfassende Betreuung. Herrn Dr. Van Tuan Tran sowie Frau Clara Hoppenau für ihre Unterstützung, Hilfestellung, und die gute und nette Atmosphäre im Labor gilt mein ganz besonders herzlicher Dank. Weiterhin möche ich mich bei Frau Maria Meyer, Herrn Kai Nesemann, sowie Frau Christiane Preiss bedanken, die als Arbeitskollegen für eine sehr freundliche und angenehme Arbeitsatmosphäre gesorgt haben.

Ebenfalls möchte ich bei Herrn Joshua Schinke, Frau Dr. Rebekka Harting Frau Dr. Jennifer Gerke, Herrn Dr. Christoph Sasse, Herrn Ronny Lehneck, Frau Kerstin Schmitt und Herrn Bastian Jöhnk für viele anregende und unterhaltsame Gespräche bedanken. Mein weiterer Dank gilt Frau Dr. Anne Dettmann, Frau Dr. Corinna Richthammer, Frau Yvonne Heilig, Frau Sarah Ludwig und Herrn Matthias Enseleit. Ein besonderer Dank gilt Herrn Dr. Özgür Bayram für viele Anregungen und Ideen im Rahmen meiner Arbeit. Frau Irene Hampe, die im Rahmen ihrer Bachelorarbeit von mir betreut wurde, möchte ich besonders danken. Ein weiterer Dank gilt Herrn Dr. Harald Kusch und Frau Anika Kühn. Ebenfalls bedanken möchte ich mich bei Frau Heidi Northemann und Frau Nicole Scheiter, die mir bei vielen organisatorischen oder bürokratischen Fragen geholfen haben.

Bei allen weiteren Mitarbeiten der Abteilung Braus möchte ich mich ebenfalls herzlich für die tolle Atmosphäre und das gute und angenehme Arbeitsklima, sowie die große Hilfsbereitschaft bedanken.

Des Weiteren möchte ich mich bei Frau Dr. Mareike Possienke und Frau Dr. Stefanie König bedanken, mit denen ich das Vergnügen hatte im Rahmen verschiedener gemeinsamer Projekte zusammenzuarbeiten. Ebenfalls möchte ich mich bei Frau Monika Franke-Klein,Frau Susanne Mester und Frau Dr. Jessica Knüfer bedanken die mir bei der Anzucht sowie der Aussaat aller Pflanzen, die ich für meine Experimente benötigt habe, geholfen haben. Weiterhin möchte ich allen Personen danken, die mich bei meiner Doktorarbeit beraten oder unterstützt haben.

Ganz besonders möchte ich mich bei meiner Familie für die Unterstützung während des gesamten Studiums und während der Anfertigung dieser Doktorarbeit bedanken. 



\section{Index of contents}

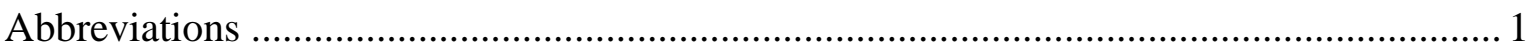

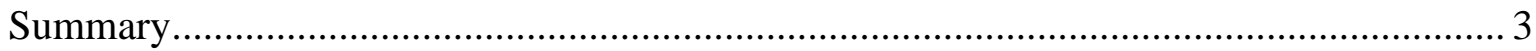

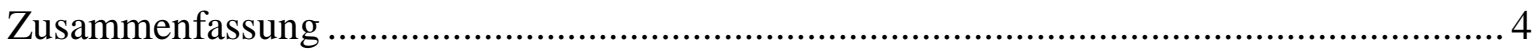

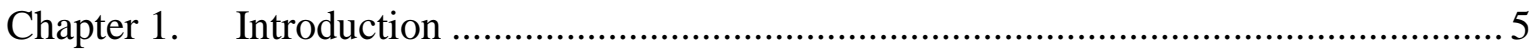

1.1 Verticillium: phytopathogenic fungi spreaded worldwide ...................................... 5

1.2. Disease symptoms by Verticillium species........................................................... 6

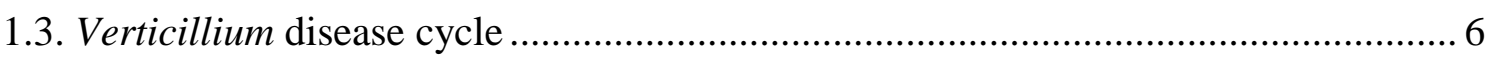

1.4. V. longisporum hybrids prefer Brassicaceae as host............................................ 9

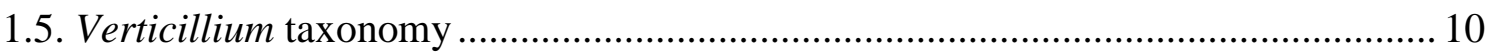

1.6. Secondary metabolism in filamentous fungi ........................................................ 14

1.7. The Fungal Cross-Pathway Control System......................................................... 16

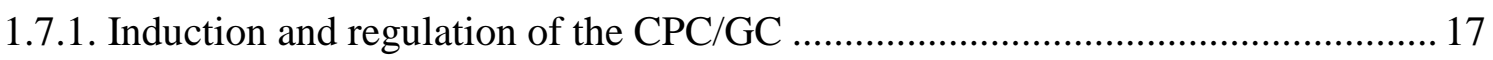

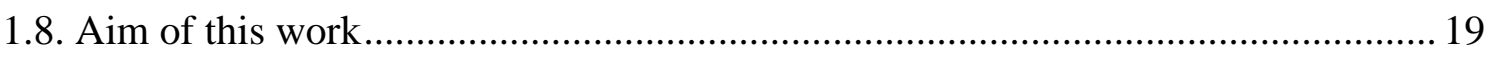

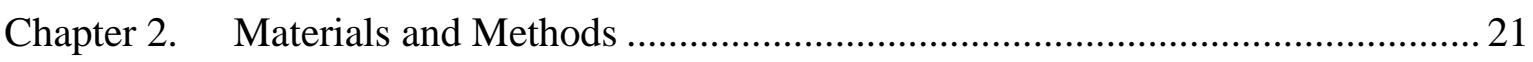

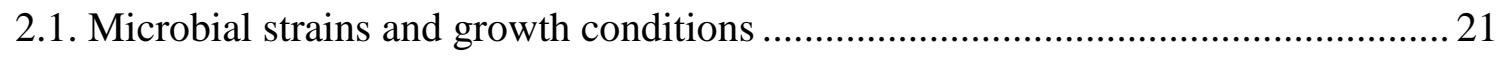

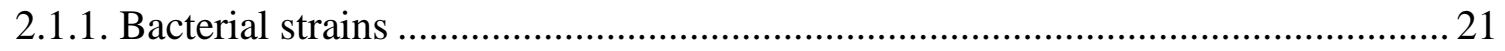

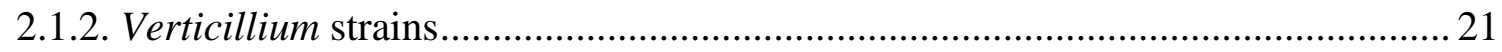

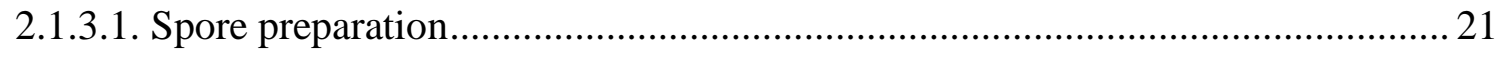

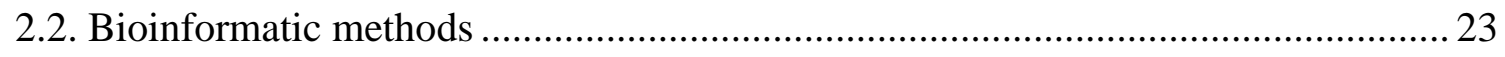

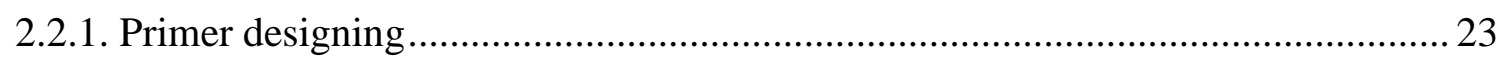

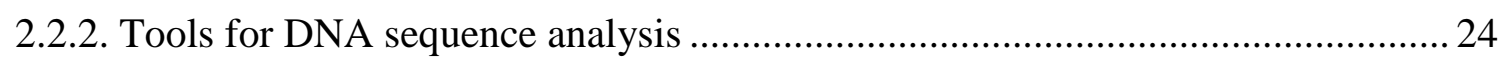

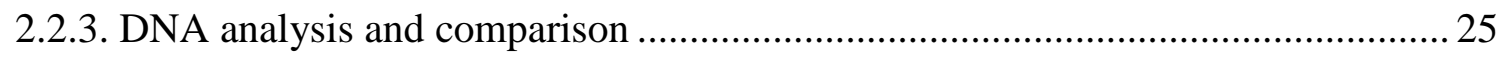

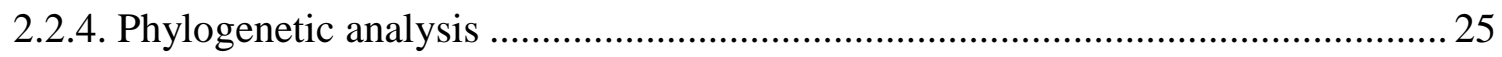

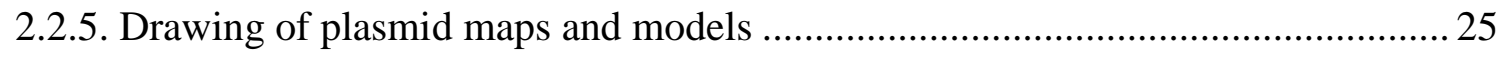

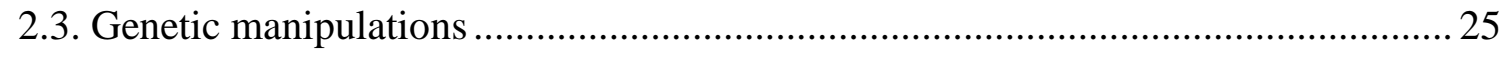

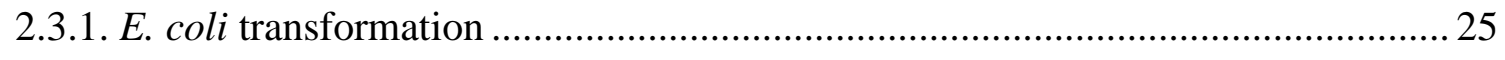

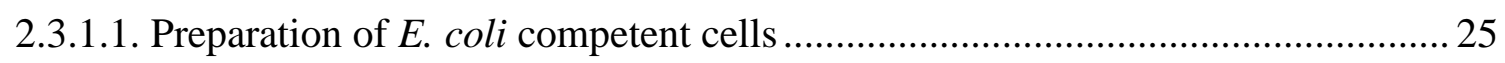

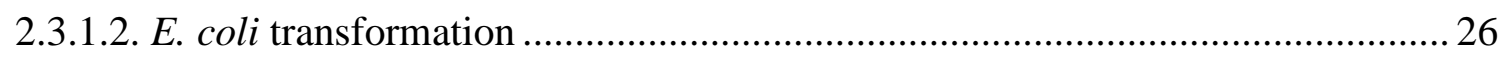

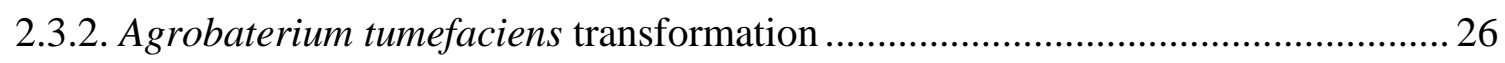

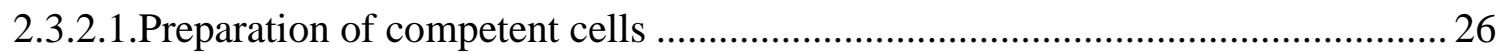

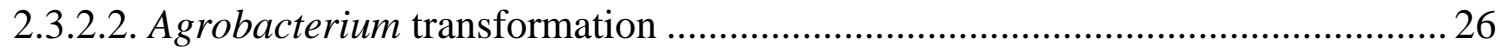

2.3.3. Agrobacterium-mediated Verticillium transformation ........................................ 27

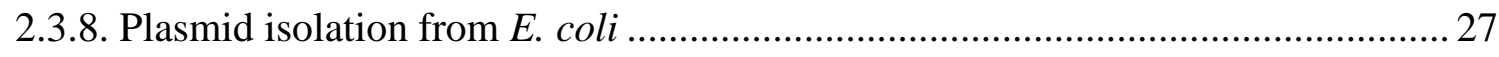




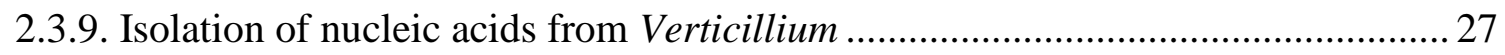

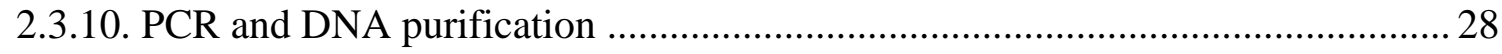

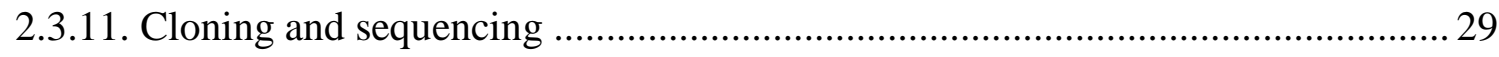

2.4. Generation of vector for gene silencing in $V$. longisporum ................................... 29

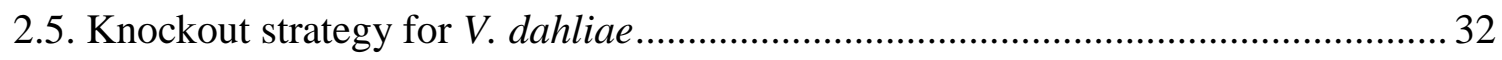

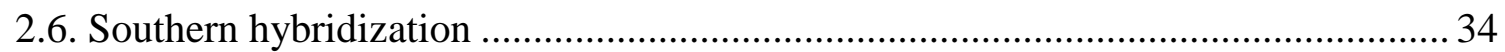

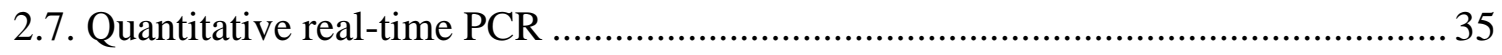

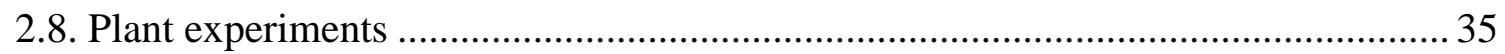

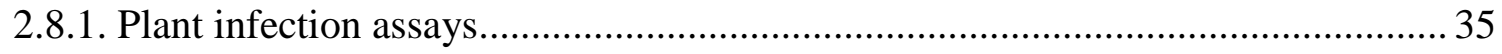

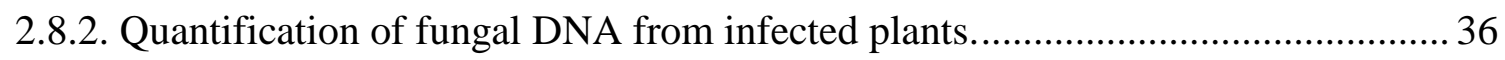

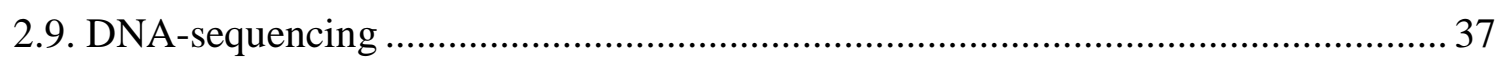

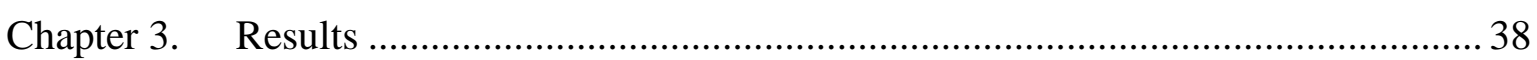

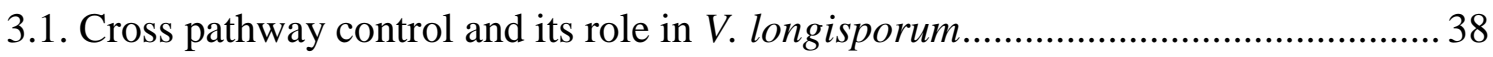

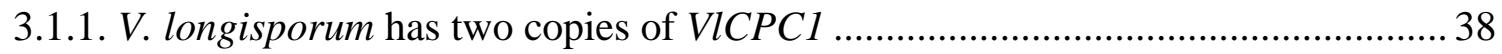

3.1.2. $\mathrm{VlCPCl}$ isogenes were silenced up to $85 \%$ by RNA-mediated gene silencing .... 46

3.1.3. Screening for knockout of $C P C 1$ in $V$. dahliae ................................................. 47

3.1.4. $\mathrm{VlCPCl}$ silenced mutants are strongly reduced in growth under amino acid

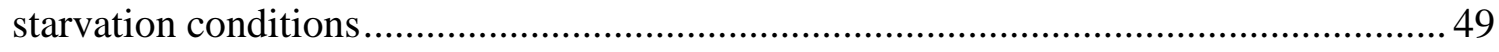

3.1.5. The saprophytic growth of $V d C P C 1$ knockout mutant is not affected.................. 54

3.1.6. Late-stage plant symptoms decrease significantly during infection in the silenced

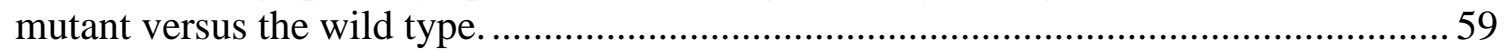

3.1.7. VdCPC1 knockout leads to reduced pathogenicity of the fungus. ........................ 65

3.2. The key regulator of secondary metabolism Lae1: a first glance in Verticillium..... 69

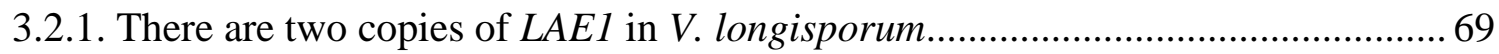

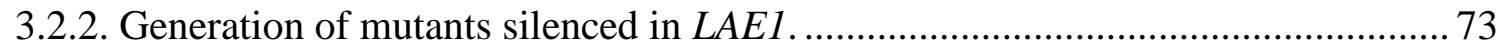

3.2.3. Silencing of $L A E 1$ reduces pathogenicity of $V$. longisporum ............................. 75

3.2.4. A deletion of $L A E 1$ doesn't affect the pathogenicity of $V$. dahliae ....................... 78

3.3. Triacylglycerol lipase (Tagl) a secreted lipase in $V$. longisporum. .......................... 80

3.3.1. V. longisporum has two copies of triacylglycerol lipase .................................... 81

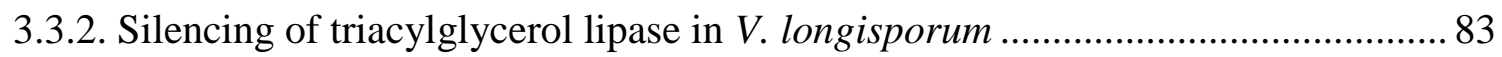

3.3.3. Silencing of triacylglycerol lipase in $V$. longisporum shows a reduced

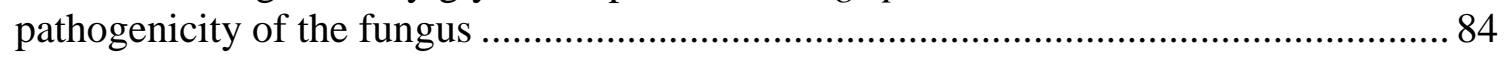

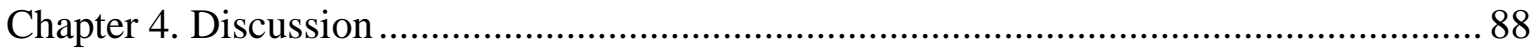

4.1. Amino acids metabolism and its role for pathogenicity......................................... 88

4.2. Cross pathway control is required for pathogenicity of $V$. longisporum .................. 89

4.2.1. Knockouts of $C P C 1$ in $V$. dahliae show similar effects than silencing in

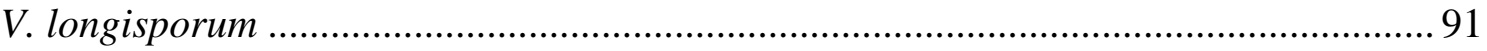

4.3. Efficiency and relieability of the silencing system.............................................. 91 


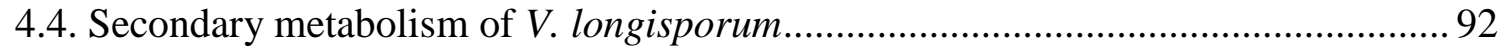

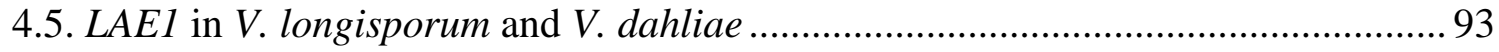

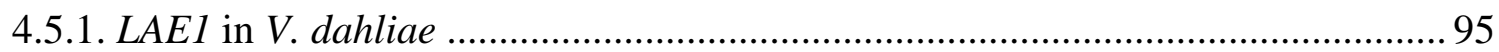

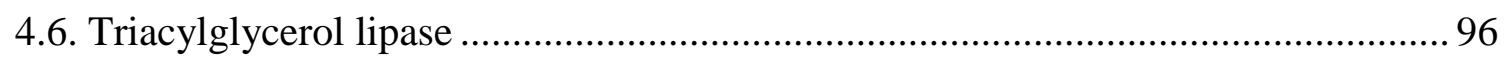

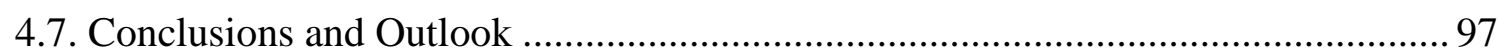

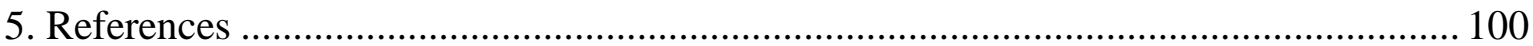




\begin{tabular}{|c|c|}
\hline AFLP & amplified fragment length polymorphism \\
\hline AmpR & ampicillin resistance \\
\hline ATMT & Agrobacterium tumefaciens-mediated transformation \\
\hline Bp & base pair \\
\hline CDM & Czapek-Dox medium \\
\hline cDNA & complementary DNA \\
\hline $\mathrm{CPC}$ & Cross-Pathway Control \\
\hline DMSO & dimethyl sulfoxide \\
\hline DNA & deoxyribonucleic acid \\
\hline E. coli & Escherichia coli \\
\hline EDTA & ethylenediaminetetraacetate \\
\hline h & hour \\
\hline $\mathrm{H}_{2} \mathrm{O}_{2}$ & hydrogen peroxide \\
\hline $\mathrm{HPH}$ & hygromycin phosphotransferase \\
\hline IGS & intergenic spacer \\
\hline ITS & internal transcribed spacer \\
\hline KanR & kanamycin resistance \\
\hline $\mathrm{kb}$ & kilobase \\
\hline LB & left border / Luria Bertani medium \\
\hline LiAc & Lithium acetate \\
\hline $\min$ & minute \\
\hline $\mathrm{ml}$ & milliliter \\
\hline $\mathrm{mM}$ & milimolar \\
\hline MM & minimal medium \\
\hline NAT & nourseothricin acetyltransferease \\
\hline ORF & open reading frame \\
\hline PCR & polymerase chain reaction \\
\hline PDA & potato dextrose agar \\
\hline PDB & potato dextrose both \\
\hline $\mathrm{RB}$ & right border \\
\hline rDNA & ribosomal DNA \\
\hline RFLP & restriction fragment length polymorphism \\
\hline RNA & ribonucleic acid \\
\hline RNAi & RNA interference \\
\hline rRNA & ribosomal RNA \\
\hline $\mathrm{s}$ & second \\
\hline S & Svedberg unit \\
\hline SDS & sodium dodecyl sulphate \\
\hline SXM & simulated xylem medium \\
\hline TE & Tris- $\mathrm{Cl}$ and EDTA \\
\hline UV & ultraviolet \\
\hline$V a$ & Verticillium albo-atrum \\
\hline VCG & vegetative compatibility group \\
\hline$V d$ & Verticillium dahliae \\
\hline$V l$ & Verticillium longisporum \\
\hline WT & wild type \\
\hline$\mu 1$ & microliter \\
\hline$\mu \mathrm{m}$ & micrometer \\
\hline
\end{tabular}




\section{Summary}

Verticillium longisporum is a soil-borne fungal pathogen of oilseed rape (Brassica napus). Infection is initiated by hyphae from germinating microsclerotia which invade the plant vascular system through penetration of the fine roots. Most of its life cycle, V. longisporum is confined to the vascular system of the plant. The xylem fluid provides an environment with limited carbon sources and imbalanced amino acid supply, which requires that $V$. longisporum induces the cross-pathway control of amino acid biosynthesis. VlCPCI encodes the conserved transcription factor of the cross-pathway control. RNA-mediated gene silencing reduced the expression of the two CPCl isogenes (VlCPC1-1, VlCPC1-2) of the allodiploid $V$. longisporum up to $85 \%$. The silenced mutants were highly sensitive to amino acid starvation and the infected plants showed significantly less symptoms such as stunting or early senescence in oilseed rape plant infection assays. Consistently, deletion of single $C P C l$ of the haploid $V$. dahliae resulted in strains, which are sensitive to amino acid starvation and cause strongly reduced symptoms in the plant-host tomato (Solanum lycopersicum). The allodiploid $V$. longisporum and the haploid $V$. dahliae are the first phytopathogenic fungi, which were shown to require $C P C l$ for infection and colonization of their respective host plants oilseed rape and tomato. We investigated secondary metabolism as it might be required for pathogenicity. The key regulator of secondary metabolism $L A E 1$ was further investigated. Silenced mutants reduced the expression of the two isogenes (VlLAE1-1, VILAEI-2) up to 80\% and showed milder symptoms on plants. The corresponding deletion in $V$. dahliae did not show any contribution to pathogenicity. Furthermore a secreted lipase was silenced as well. The efficiency of silencing reached around $80 \%$ and the mutants showed a reduced pathogenicity on oilseed rape in plant infection assays. 


\section{Zusammenfassung}

Verticillium longisporum ist ein bodenbürtiger pathogener Pilz, welcher Raps (Brassica napus) befällt. Eine Infektion geht von Hyphen aus, die aus keimenden Mikrosklerotien entstanden sind und die Pflanze über Penetration der Wurzelhaare infizieren. Der Lebenszyklus von V. longisporum ist zu einem Großteil auf das vaskuläre System der Pflanze beschränkt. Xylemsaft stellt einen Lebensraum mit eingeschränkten Kohlenstoffquellen und einer unausgewogenen Aminosäureversorgung dar. Unter diesen Bedingungen aktiviert $V$. longisporum das Gen für den Regulator der „,cross pathway control“ für Aminosäurebiosynthesen. Über RNA-vermitteltes Gensilencing konnte die Expression der beiden CPCl Isogene (VlCPC1-1, VlCPC1-2) für diesen Regulator im allodiploiden Pilzes V. longisporum um $85 \%$ verringert werden. VlCPCl codiert den konservierten Transkriptionsfaktor der cross pathway control. Die gesilencenten Mutanten reagierten sehr empfindlich auf Aminosäuremangelbedingungen und die infizierten Pflanzen zeigten schwächer ausgeprägte Symptome der Krankheit wie Stauchung und frühere Reifung in den durchgeführten Pflanzeninfektionsreihen mit Raps. Eine Deletion von CPC1 im haploiden $V$. dahliae generierte Stämme, welche sensitiv auf Aminosäuremangelbedingungen reagieren mit stark verringerten Symptome auf seine Wirtspflanze Tomate (Solanum lycopersicum). Der allodiploide V. longisporum und der haploide $V$. dahliae sind die ersten phytopathogenen Pilze in denen gezeigt werden konnte, dass CPC1 für Infektion und Kolonisierung ihrer jeweiligen Wirte benötigt wird. Der Sekundärmetabolismus des Pilzes könnte ebenso eine Rolle für die Pathogenität spielen. Der Regulator des Sekundärmetabolismus LAE1 wurde weitergehend untersucht. Die Expression der beiden Isogene in V. longisporum (VILAE1-1, VILAE1-2) wurde um 80\% reduziert und zeigten verringerte Symptome auf Raps. Die entsprechende Deletion in $V$. dahliae zeigte keinen Beitrag zur Pathogenität des Pilzes. Weiter wurde eine Lipase identifiziert, die vom Pilz im Xylemsaft sezerniert wird. Diese sekretierte Lipase konnte ebenso gesilenced werden. Die Effizienz lag bei $80 \%$ und die Mutanten zeigten eine verringerte Pathogenität in Pflanzeninfektionsreihen auf Raps. 


\section{Chapter 1. Introduction}

\subsection{Verticillium: phytopathogenic fungi spreaded worldwide}

The Verticillium species are soil-borne plant pathogenic fungi. They are widely spread around the world and cause so-called vascular wilting diseases and early senescence in a broad number of economically important crops including alfalfa, cotton, lettuce, hops, olive trees, oilseed rape, cabbages, potato, tomato and strawberries. Verticillium dahliae, $V$. albo-atrum and $V$. longisporum are the species which cause the highest losses of crops (Pegg and Brady, 2002; Zeise and von Tiedemann, 2002a; Zeise and von Tiedemann, 2002b; Agrios, 2005). The name Verticillium is based on the phialides arrangement in whorls (verticillate shape) around the conidiophores and branching of the conidiophores also occurs in whorls at some levels (Fig. 1). The fungal mycelium is hyaline, simple or branched, septated and multinucleated. Each phialide carries a number of ovoid to elongated conidia (Berlanger and Powelson, 2000; Fradin and Thomma, 2006). The first Verticillium strain was detected in 1879 by Reinke and Berthold, who investigated wilt on potato, and isolated the causal agent, which was named Verticillium albo-atrum (Reinke and Berthold, 1879; Hastie, 1973; Klosterman et al., 2009).

In 1913 Klebahn described a second species causing wilt on dahlia (Asteraceae family) named V. dahliae with morphological distinction (Isaac, 1947; Hastie, 1973). In 1961, Stark isolated a $V$. dahliae-like fungus from horseradish in Hamburg, Germany (Stark, 1961). This fungus was named $V$. dahliae var. longisporum. It produces microsclerotia like $V$. dahliae but conidia are significantly longer than those of typical $V$. dahliae strains. Therefore it was named $V$. dahliae var. longisporum. Besides the fact that isolates from crucifers produced longer conidia, as had already been noted by Stark (1961), they found additional differences with regard to morphological, enzymatic, molecular and virulence characteristics (Fig. 1). On the basis of the morphological differences and other characteristics of many similar strains, Karapapa et al. suggested long-spored isolates as a new species, V. longisporum (Karapapa et al., 1997). Since scientists have worked with this fungus, there has been a controversy concerning the recognition of $V$. longisporum as a separate host-specific species. Currently, the genomes of $V$. dahliae and $V$. albo-atrum have been sequenced by the Broad Institute, Harvard-Massachusetts (Klosterman et al. 2009). Sequencing of $V$. longisporum genome is in progress by the Biofung project at the University of Göttingen. 
A
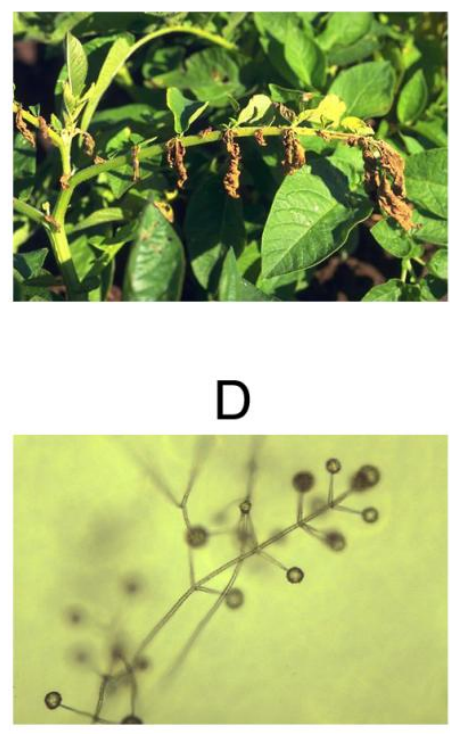

B

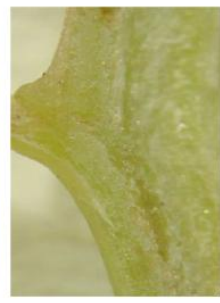

C
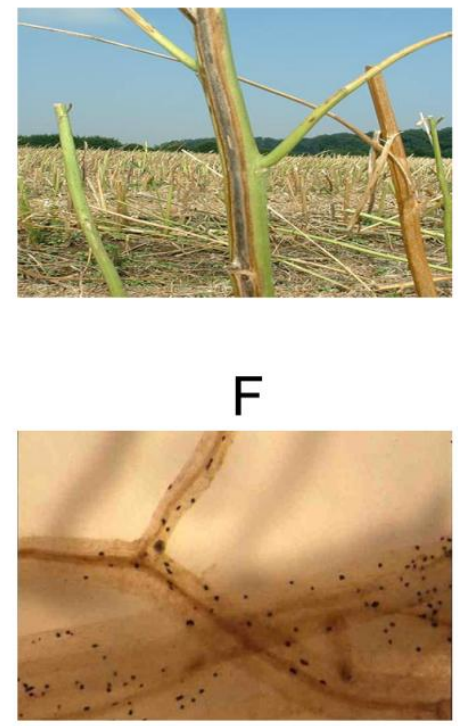

Fig. 1. Wilt disease symptoms and morphological structures of Verticillium pathogens. (adapted from Heale and Karapapa, 1999; Berlanger and Powelson, 2000; Andrie et al., 2005; Eynck et al., 2009). (A) Leaf necrosis and wilt in potato. (B) Longitudinal section through the stem of a healthy spinach plant (left) and a spinach plant infected with Verticillium dahliae (right). (C) Necrotic symptom of infected rapeseed stems with black microsclerotia. (D) Verticillium conidia masses on phialides arranged in whorls (verticillate) around conidiophores. (E) The survival structure as microsclerotia of $V$. dahliae on infected potato stems. (F) Microsclerotia of $V$. longisporum on infected rapeseed.

\subsection{Disease symptoms by Verticillium species}

In comparison with Fusarium wilt symptoms induced by Verticillium are quite similar and difficult to observe in the field (Babadoost et al., 2004). Symptoms caused by the fungus develop steadier and mainly on the lower and outer parts of plants. Since indicators for disease can vary between different plant hosts, no absolute diagnostic method for Verticillium has been established (Rowe and Powelson, 2002). Anyhow infection with Verticillium shows some characteristics, like vascular discoloration in stems and roots and premature foliar chlorosis and necrosis. In some hosts, older plants infected with Verticillium are usually stunted in various degrees (Kim et al., 2001; Pegg and Brady, 2002; Rowe and Powelson, 2002; Fradin and Thomma, 2006; Gladders et al., 2011).

\subsection{Verticillium disease cycle}

Verticillium wilt is a monocyclic disease, as there is only one cycle of disease per season (Fig. 2). V. longisporum life cycle is similar to that of other Verticillium species and can be 
divided into a dormant, a parasitic and a saprophytic phase. To survive in the soil $V$. dahliae and $V$. longisporum have developed microsclerotia as resting structure, whereas V. albo-atrum forms resting mycelium (Karapapa et al., 1997; Rowe and Powelson, 2002; Eynck et al., 2007). Microsclerotia are melanized aggregates of enlarged hyphal cells (Schnathorst, 1981; Heale and Karapapa, 1999). These structures are generated under growth limiting conditions, and remain viable in the soil for several years (Schnathorst, 1981; Heale and Karapapa, 1999); up to 14 years for microsclerotia (Wilhelm, 1955) and around 2-5 years for resting mycelium (Sewell and Wilson, 1964). To compete in the soil ecosystem microsclerotia must withstand microbial degradation, lysis, parasitism and predation, and therefore present an important nutrient state available in the soil (Okubara and Paulitz, 2005). Under favorable conditions microsclerotia can start to germinate again, usually after stimulation with root exudates or contact with the host plant (Zhou et al., 2006; Eynck et al., 2007). These hyphae originating from resting structures grow towards the roots of the plants, and infect through the root tips or follow the root hairs. After breaching the cell wall, fungal hyphae grow through cortical tissues towards developing vascular tissue (Zhou et al., 2006; Eynck et al., 2007). 
Spreading of the pathogen throughout the internal transport system of the plant
Appearance of the first wilting symptoms in leaves, roots, main and lateral shoot

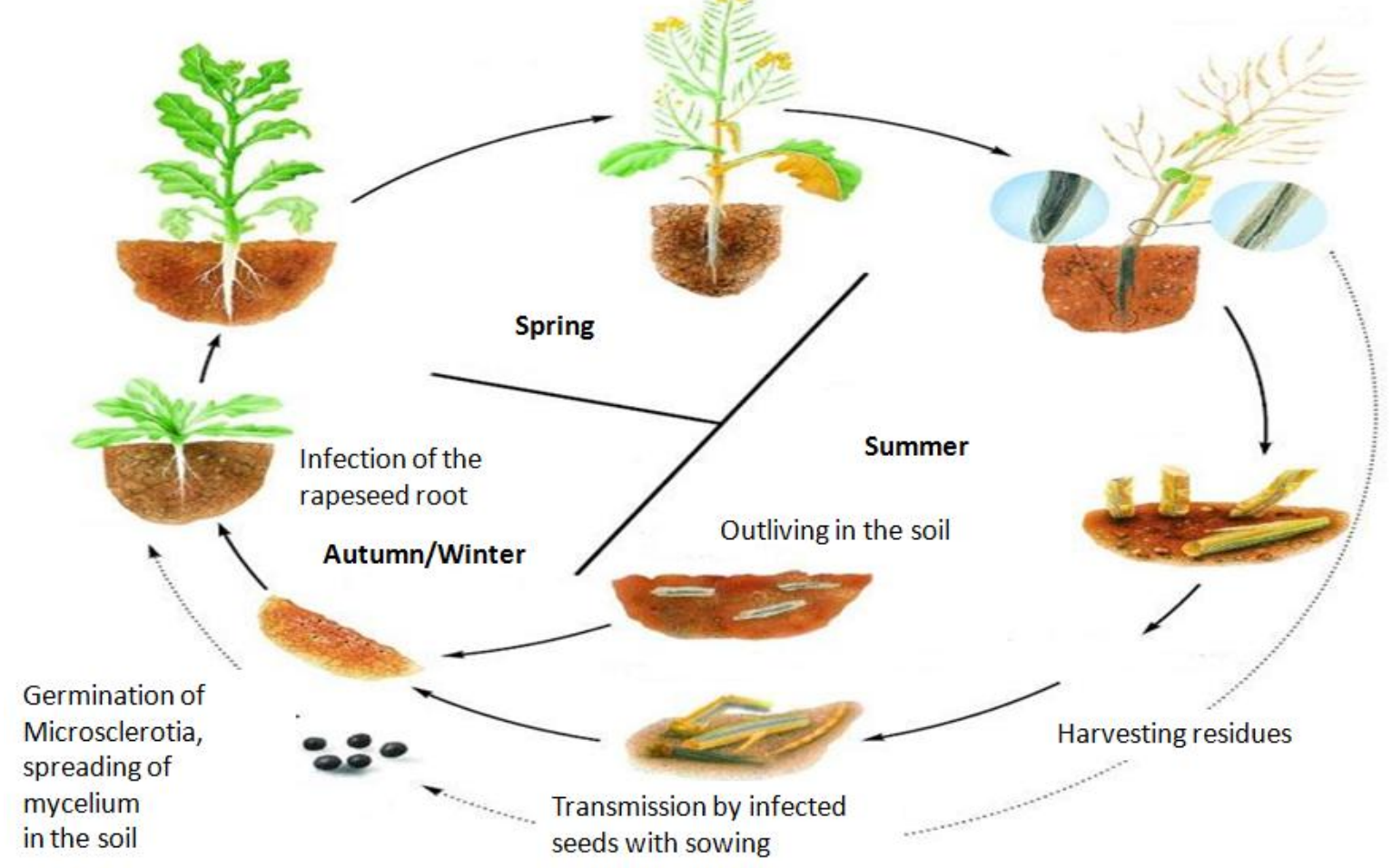

Fig. 2 . The life cycle of $\boldsymbol{V}$. longisporum on oilseed rape (modified from Paul, 2003). Like $V$. dahliae, the infection cycle of $V$. longisporum starts with germination of microsclerotia under the stimulation of root exudates. The fungus enters the plant through the root hairs and grows in the plant vascular system until the first symptoms of stunting and chlorosis can be observed. When the plant becomes old, the fungus produces microsclerotia in plant roots and shoots. These resting structures can be released from dead plant materials into the soil for the next cycle. Microsclerotia can survive in the soil for several years without rapeseed plants.

The fungus colonizes the vascular system and does not leave this environment until senescence and maturity stages of the host plant. The fungal proliferation in this environment is primarily obtained by spore release because colonization by vegetative growth would be too slow due to the poor mycelial growth rate (Presley et al., 1966). Conidia are produced within the xylem vessels and move along with the transpiration stream. Thereafter colonization is pursued via germination of conidia and penetration of germ tubes through full-bordered pits into adjacent vessel elements (Garas et al., 1986), and that way starting another infection cycle. This kind of host colonization may represent on the one hand the observed discontinuous occurrence of mycelium in the plant vascular system (Beckman, 1987; Heinz et al., 1998) and on the other hand the rapidity with which systemic colonization occurs (Zinkernagel, 1982; Gold and Robb, 1995; Heinz et al., 1998; Chen et al., 2004). Consequently, the fungus emerges from the xylem vessels to colonize 
neighboring vascular and cortical tissues, resulting in the development of disease symptoms such as wilting, chlorosis and necrosis.

With the progression of disease development, senescence of the host begins, and the pathogen enters a limited saprophytic growth phase in which microsclerotia are formed in the dying stem parenchyma (Schnathorst, 1981; Neumann and Dobinson, 2003). Unlike other Verticillium species, V. longisporum does not induce wilt symptoms in oilseed rape grown on the field. The colonization of the plant is characterized by an extended latent phase, early symptoms like streaky yellow to brownish discolorations of the stem and halfsided yellowing of the leaves as well as chlorotic patches between veins which themselves turn blackish, are not monitored before the beginning of plant maturity. Past the initiation of maturity the fungus starts to advance out of the xylem vessels and starts to generate large numbers of microsclerotia underneath the stem epidermis, in the stem pith and in the roots. Further symptoms of the disease are premature bloom and maturity as well as stunted growth, the latter occurring particularly under standardized conditions in the greenhouse or climate chamber. Regarding these considerations, a more appropriate term to describe the disease caused by $V$. longisporum on crucifers would rather be Verticillium premature senescence (VPS) or Verticillium premature ripening (VPR) (Eynck et al., 2009) than Verticillium wilt. Resistance to Verticillium according to Pegg \& Brady (2002) can be defined as the total or partial absence of symptoms in comparison with other host species or cultivars similarly exposed to virulent pathotypes and showing severe damage or death`. The systemic nature of Verticillium infections makes it difficult to quantify the cellular bases of resistance and susceptibility to wilt disease (Gold and Robb, 1995).

\subsection{V. longisporum hybrids prefer Brassicaceae as host}

Due to the limitation of natural resources the demand for bio fuels has been rising within the last ten years, and has increased the cultivation of oil producing plants. In our days oilseed rape is one of the most important oil sources, next to soybean and cottonseed. The opposite site of this development is the progression of pathogens like $V$. longisporum (Heale and Karapapa, 1999) (Heale and Karapapa, 1999; Pua and Douglas, 2004). Infection of Brassica crops with this fungus has been reported for many European countries like Germany, France, Poland and Sweden, but it has also been announced for Canada (Svenson and Lerenius, 1987; Zielinski and Sadowski, 1995; Karapapa et al., 1997; Zeise and von Tiedemann, 2002a; Dixelius et al., 2005). V. longisporum is a soilborne fungal pathogen and host-specific on the Brassicaceae family such as oilseed rape 
(Brassica napus), cabbage (Brassica oleracea var. capitata), horseradish (Armoracia rusticana), cauliflower (Brassica oleracea var. botrytis). Though infection with $V$. longisporum does not cause true wilting symptoms on oilseed rape, however premature senescence and ripening are accompanied by systemic spread and extensive formation of microsclerotia on shoot tissue. This can cause yield reductions up to 50-70\%. In greenhouse experiments chlorosis and stunting are mainly applied to determine the disease development, but in field studies stunting has not been observed so far (Dunker et al., 2006). Some greenhouse pathogenicity assays with Brassica crops showed that $V$. longisporum isolates are most virulent, whereas $V$. dahliae strains are non-pathogenic or weakly virulent (Zeise and von Tiedemann, 2002a). V. longisporum infects mainly oilseed rape causing losses in plant fresh weight of $49 \%$ and killing about half of the plants at 42 days post inoculation (Zeise and von Tiedemann, 2002a). This pathogen can also infect some other non-host plants (Bhat and Subbarao, 1999; Fahleson et al., 2004; Johannson et al., 2005) and conversely, other Verticillium species also weakly infect B. napus (Zeise and von Tiedemann, 2002a; Collins et al., 2003).

\subsection{Verticillium taxonomy}

The morphology of Verticillium implies characteristic verticilliate arrangement of the three to five asexual spore carrying structures (phialides) forming branches at each node of the conidiophores (Kim et al., 2001). V. dahliae and V. albo-atrum are two closely related but distinct mature species. $V$. dahliae forms microsclerotia as a resting form which are melanized clumps formed by budding of mycelial cells (Goud et al., 2003), whereas V. albo-atrum forms melanized resting mycelium. To distinguish $V$. dahliae and $V$. albo-atrum the most common feature is the formation of these resting structures (Pegg and Brady, 2002). Futhermore V. albo-atrum is not able to grow in cultures or to infect its hosts at temperatures of $30^{\circ} \mathrm{C}$, while $V$. dahliae still grows and infects plant (Rowe and Powelson, 2002). Via morphological description and rDNA isolates, V. albo-atrum can be divided into two groups, Grp1 and Grp2. Most V. albo-atrum strains are referred to Grp1 (group1) (Robb et al., 1993; Morton et al., 1995; Barbara and Clewes, 2003; Robinson et al., 2007; Klosterman et al., 2009). Morphological analysis showed that Grp2 (group2) isolates produce resting structures in bundles of melanized hyphae, whereas Grp1 isolates form melanized single hyphae (Mahuku and Platt, 2002). When analysing the sequence of the ITS region it could be demonstrated that the ITS of Grp2 isolates contain 17 bases that 
are not found in the Grp1 isolates (Robb et al., 1993; Mahuku and Platt, 2002). The genus Verticillium includes four other species: $V$. tricorpus, $V$. nubilum, $V$. nigrescens and V. theobromae (Barbara and Clewes, 2003). In contrast to V. dahliae and V. albo-atrum, $V$. tricorpus and V. nubilum are soil saprophytes that can grow in the absence of a potential host (Isaac and Milton, 1967). V. tricorpus is regarded as a weak pathogen on many hosts and produces survival structures including chlamydospores, microsclerotia and melanized hyphae (Robinson et al., 2007; Qin et al., 2008), whereas V. nubilum produces only chlamydospores as the resting structure (Griffiths, 1982; Barbara and Clewes, 2003). The species $V$. nigrescens and $V$. theobromae that are similar to other Verticillium species in morphology have been recently classified into the genera Gibellulopsis and Musicillium, according to the molecular evidences from rDNA (Zare et al., 2007). In addition, $V$. lecanii, an entomopathogen and $V$. fungicola, a pathogen of mushrooms, were assigned to the genus Lecanicillium (Zare and Gams, 2008).

V. longisporum was first isolated from horseradish (Stark, 1961), it can be distinguished to the other Verticillium species by the number of phialides and the form of microsclerotia. The fungus generates preferentially only three phialides per node and survives by means of black but compared to $V$. dahliae elongated microsclerotia that are arranged in a bead like structure (Fig. 3). Conidia of $V$. longisporum are twice as long (7-9 $\mu \mathrm{m})$ as those of V. dahliae (3.5-5.5 $\mu \mathrm{m}$ ) (Subbarao et al., 1995; Karapapa et al., 1997; Zeise and von Tiedemann, 2001; Collins et al., 2003). Isolates of $V$. longisporum have been collected from crucifers throughout the whole world like horseradish from Illinois (Eastburn and Chang, 1994), oilseed rapes from Europe and Canada (Heale and Karapapa, 1999; Zeise and von Tiedemann, 2001; Steventon et al., 2002; Johannson et al., 2005) or cauliflower from California (Koike et al., 1994). Although many isolates have been closely investigated, the taxonomy of $V$. longisporum is still controversially discussed (Klosterman et al., 2009; Inderbitzin et al., 2011). Currently, the sexual stage has not been found in the genus Verticillium (Pegg and Brady, 2002; Klosterman et al., 2009). 

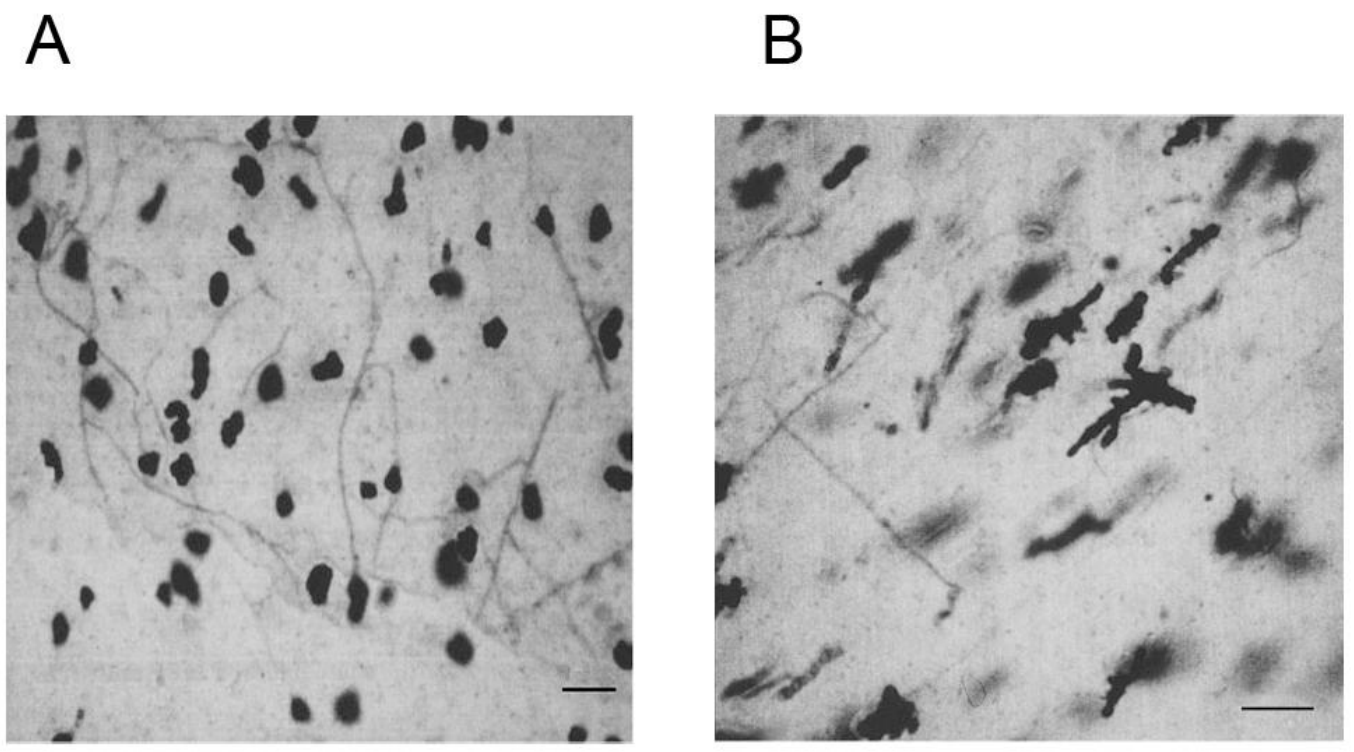

Fig. 3 Microsclerotia of $\boldsymbol{V}$. dahliae and $\boldsymbol{V}$. longisporum. (adapted from Karapapa et al. 1997) A. Mature, compact, spherical microsclerotia on rape medium of $V$. dahliae. B. Mature, irregular, elongate (much less compact) microsclerotia on oilseed rape medium of $V$. longisporum.

Based on sequence data Fahleson et al. (2004) suggested that $V$. longisporum should be regarded as a distinct species closely related to $V$. albo-atrum. Regarding the results of other authors, they conceded that the name $V$. longisporum might be somehow misleading because within the new species isolates with relatively short conidia could be grouped with long-spored isolates, at least on the molecular level (Steventon et al., 2002), and a variety of different spore lengths could be observed for the same isolate (Subbarao et al., 1995; Collins et al., 2003). As a matter of fact, in the opinion of the authors, the host specifity of $V$. longisporum is not limited to only Brassica host plants as several other Verticillium species can use especially very weak plants, as a host, and $V$. longisporum can also infect plant species outside the Brassicaceae family (Johannson et al., 2003; Johannson et al., 2005). This latter statement contradicts the results of several investigations according to Zeise \& von Tiedemann (2001, 2002a, 2002b) who showed that $V$. longisporum is a strictly host adapted pathogen specific for Brassica species. Additional confusion has been caused by some misidentifications of $V$. dahliae and $V$. longisporum. In several publications, $V$. dahliae has been considered to be the causal agent of Verticillium wilt in Brassica crops (Xiao and Subbarao, 2000; Söchting and Verreet, 2004) or on horseradish (Babadoost et al., 2004), without considering that long-spored isolates may have been involved. On the basis of microsatellite and other marker analyses, Barbara and colleagues (Barbara and Clewes, 2003; Barbara et al., 2005; Clewes and Barbara, 2005) addressed the 
parental origin of the interspecific hybrid Verticillium isolates. They suggested that longspored crucifer isolates have emerged through parasexual hybridisation between a species that is probably generally similar to other haploid $V$. dahliae isolates and one that is 'V. albo-atrum-like' but clearly distinct and of unknown morphology.

$V$. longisporum was defined as 'near-diploid' or amphihaploid fungus with higher nuclear DNA amounts (about 1.8 times) than those of $V$. dahliae or $V$. albo-atrum isolates (Karapapa et al., 1997; Steventon et al., 2002; Collins et al., 2003). This almost diploid status might be the reason why numerous mutagenesis approaches have failed (Ingram, 1968; Hastie, 1973; Nagao et al., 1994; Subbarao et al., 1995; Karapapa et al., 1997; Zeise and von Tiedemann, 2001; Steventon et al., 2002; Collins et al., 2003). Most filamentous ascomycetes are primarily haploid. Experimental studies with the model fungus A. nidulans suggest that during adaptation to a novel environment, haploids deriving from diploids by parasexual recombination reach a higher fitness than the original diploids (Schoustra et al., 2007). V. longisporum might therefore represent a nascent species due to changes in ploidy where the subsequent reduction of the genome size has just started. Speciation might not even be accomplished in $V$. longisporum, because shortspored crucifer isolates might be haploid recombinants of long-spored isolates and distinct from non-crucifer isolates of $V$. dahliae (Barbara and Clewes, 2003; Collins et al., 2003; Qin et al., 2006; Clewes and Barbara, 2008; Klosterman et al., 2009). Increase in ploidy is achieved by a hybridization event between two haploid nuclei resulting in a diploid nucleus. Haploidization requires mitotic recombination and a gradual reduction of the genome by chromosome loss due to nondisjunction during mitosis. The fusion of two haploid nuclei can either happen in a homokaryon or in a heterokaryon. Heterokaryon formation is the result of parasexuality between two different compatible Verticillium species with different nuclei. Although fusion of nuclei of the same species is not totally excluded (Clewes and Barbara, 2008), it seems likely that the fusion of different haploid nuclei to a heterozygous diploid interspecies hybrid had been the initiation event for $V$. longisporum formation that ultimately resulted in broadening the host range. Amplified fragment length polymorphism (AFLP) and restriction fragment length polymorphism (RFLP) support $V$. longisporum as interspecies hybrid between $V$. dahliae and V. albo-atrum (Karapapa et al., 1997; Steventon et al., 2002; Collins et al., 2003). The internal transcribed spacer 2 (ITS2) region of nuclear ribosomal DNA as well as mitochondrial genes suggest a closer relationship of $V$. longisporum to $V$. albo-atrum than to $V$. dahliae (Fahleson et al., 2004). In contrast sequence comparison of the beta-tubulin, 
histone 3 and 5S rRNA genes favours an interspecific hybrid between $V$. dahliae and one or even two yet unidentified species excluding $V$. albo-atrum as parents of $V$. longisporum(Clewes and Barbara, 2008; Collado-Romero et al., 2010; Inderbitzin et al., 2011; Tran et al., 2013). Recent studies analysed eight genetic loci including the ribosomal internal transcribed spacer (ITS) regions and genes for two structural proteins (actin, tubulin), two enzymes (glyceraldehyde-3-P-dehydrogenase, tryptophan synthase), the genes for the mating types (MAT1-1), one transporter (mitochondrial oxalacetate transport protein) and one translation factor (EF1- $\alpha)$ (Inderbitzin et al., 2011). These studies proposed that $V$. longiporum hybridized at least three times resulting in three different lineages, A1xD1, A1xD2, A1xD3 (Fig. 4). Species A1xD2 can only be found in the USA, while $\mathrm{A} 1 \mathrm{xD} 1$, and $\mathrm{A} 1 \mathrm{xD} 3$ have as well been isolated in Europe and Asia. All three lineages share the same A1 parental genome (Inderbitzin et al., 2011; Tran et al., 2013). The A1 and D1 had been described as yet unknown Verticillium species, whereas D2 and D3 represent $V$. dahliae lineages (Inderbitzin et al., 2011). V. longisporum A1xD1 and A1xD3 hybrids were isolated from oilseed rape in Europe which are virulent or avirulent, respectively, but differ in the second parental genome by several single nucleotide polymorphisms (SNPs) and ribosomal DNA type (Zeise and von Tiedemann, 2001; Tran et al., 2013).

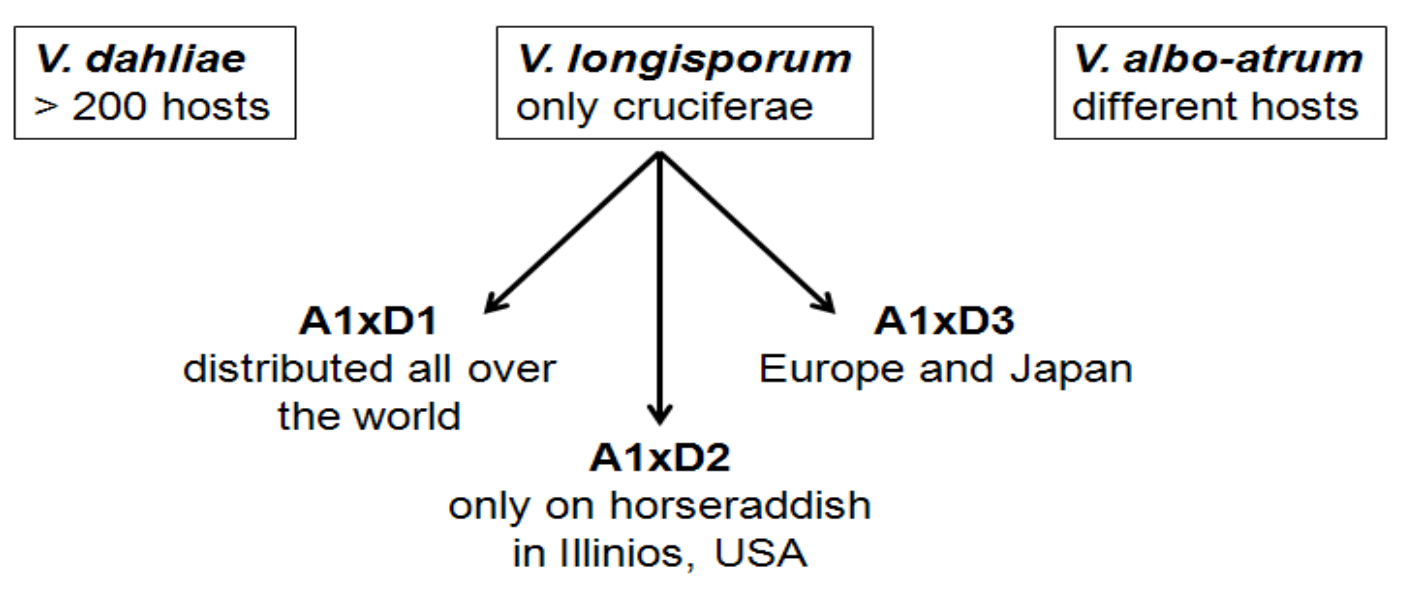

Fig. 4 Schematic distribution of Verticillium plant pathogenic species The haploid species V. dahliae and $V$. albo-atrum infect a broad variety of plant host while $V$. longisporum has a narrow host range only infecting cruciferous plants. V. longisporum has three different lineages including A1xD1, A1xD2 and A1xD3 with the same A1 parent genome. (adapted from Pegg and Brady, 2002; Inderbitzin et al., 2011; Tran et al., 2013).

\subsection{Secondary metabolism in filamentous fungi}

Secondary metabolites are defined as compounds or substances, which are produced by an organism but are not required for primary metabolic processes (Mann, 1986). Fungi have a 
huge capability of producing different secondary metabolites, including pharmaceutically important compounds such as antibiotics, as well as mycotoxins that cause poising reactions in animal and plant tissues ( $\mathrm{Yu}$ and Keller, 2005). Expression of secondary metabolites influences competitive advantage of fungi (Cox, 2007). The metabolites are expressed along with enzymes necessary for extracellular digestion. However, the precise function of many of these compounds in the natural environment is currently unknown. Some of these substances have an influence on the organisms interacting with the fungus (Mattinen et al., 2007; Kulye et al., 2012), and some others like toxins are substances with the potential to kill organisms (Sweeney and Dobson, 1999; Mayer et al., 2001; Taborda et al., 2008; Kulye et al., 2012).

Secondary metabolites are generally produced following active growth, and many have an unusual chemical structure (Vining, 1990). Some metabolites are widely spread throughout different fungal groups, while others are specialized for only one or a few species (Muller and Hausmann, 2011).

Secondary metabolites of fungi are classified into four groups depending on the chemical properties and the manner of synthesis (Keller et al., 2005). The main groups are polyketides, peptides, alkaloids and terpenes. Polyketides represent the biggest group of fungal secondary metabolites including the yellow A. nidulans spore pigment intermediate naphthopyrone (WA), the carcinogen aflatoxin (aftatoxin $\mathrm{B}_{1}, \mathrm{~B}_{2}, \mathrm{G}_{1}$ and $\mathrm{G}_{2}$ ) and the cholesterol-reducing compound lovastatin. Penicillin G, cyclosporin and gliotoxin are representatives of the group of non-ribosomal peptides (NRP). Terpenes like aristolochenes, caretenoids, gibberellins, trichothecenes are compounds that consist of isoprene units. Indole alkaloids are the derivatives of tryptophan and dimethylallyl pyrophosphate, gibberellin GA3, trichothecene T2 toxin and aristolochene (Keller et al., 2005).

The fungus Aspergillus nidulans serves as a model system to investigate secondary metabolism in the genus Aspergillus. It can produce the polyketide sterigmatocystin (ST) and aflatoxins (AF) which are related fungal secondary metabolites (Keller and Adams, 1995; Keller and Hohn, 1997). They are among the most toxic, mutagenic and carcinogenic natural products known (Trail et al., 1995; Sweeney and Dobson, 1999). The genes involved in ST biosynthesis are placed in a cluster (Brown et al., 1996). Structural genes involved in fungal secondary metabolism act as one locus (Bok et al., 2006).

Clustered genes are mainly regulated by pathway specific transcription factors (Fernandes et al., 1998; Pedley and Walton, 2001), global regulators (Bok and Keller, 2004) and signal 
transduction pathways that for example combine the secondary metabolism with sporulation (Calvo et al., 2002). Transcription of the ST gene cluster is driven by aflR, which is the transcription factor embedded within the ST structural gene cluster (Fernandes et al., 1998). Further upstream regulation is conferred by LaeA, which is the master regulator of secondary metabolism in Aspergillus spp. It is a methyltransferase possibly acting on histone methylation (Keller et al., 2005). In A. nidulans, deletion of laeA causes the loss of expression of ST gene cluster including the transcription factor aflR (Bok and Keller, 2004), A. fumigatus laeAs strains have decreased virulence in a murine model (Bok et al., 2005). The regulation of fungal secondary metabolism is controlled by many factors and is depending on a specific stage of fungal development. LaeA is a part of the velvet complex including VelB-VelA-LaeA that controls development and secondary metabolism (Bayram et al., 2008). Velvet proteins regulate development and secondary metabolism in A. nidulans and other fungi (Bayram and Braus, 2012).

Secondary metabolism in Verticillium is still largely unexplored. Although some common gene clusters for secondary metabolism have been identified, knowledge about their function is limited (Wang et al., 2004; Shi and Li, 2008; Zhou et al., 2012). For V. dahliae an elicitor that induces cotton wilting was isolated (Wang et al., 2004). Several of these elicitors have been identified so far and they are related to induction of wilting symptoms in cotton plants (Zhou et al., 2012). Futhermore toxins generated by $V$. dahliae charge a counter reaction of the plant via nitric oxygen (Shi \& Li, 2008). Some investigations on interaction between plant and fungus on proteomic or on genetic level revealed putative secondary metabolites (Singh et al., 2010; Floerl et al., 2012; Iven et al., 2012; Singh et $a l ., 2012)$, but so far no deeper insight into secondary metabolism have been described.

\subsection{The Fungal Cross-Pathway Control System}

Changing environmental conditions are a challenge for microorganisms, as they have to adapt to certain conditions. To increase their chances to survive under rapidly evolving environmental situations, they must have a system that is on the one hand sensitive and that on the other hand enables them to quickly respond on these impacts. Such kinds of regulatory systems were detected in various fungi like the yeast Saccharomyces cerevisisae (Hinnebusch, 1984) or in filamentous moulds like Neurospora crassa and Aspergillus nidulans (Carsiotis et al., 1974; Piotrowska et al., 1980; Sachs, 1996; Davis, 2000) where they were named "General Control of amino acid biosynthesis" (GC) or "Cross-Pathway Control" (CPC), respectively. They are activated under amino acid 
starvation conditions and result in a regulation of different sets of genes involved in amino acid biosynthesis, purine biosynthesis, nitrogen and sulfur metabolism and vitamin biosynthesis (Mirande and Waller, 1988; Mösch et al., 1991; Hinnebusch, 1997; Natarajan et al., 2001; Tian et al., 2007). In its core, this signal transduction system consists of a sensor kinase, which is able to phosphorylate a subunit of the eukaryotic translation initiation factor eIF2, and a transcriptional activator that serves as downstream effector to generate a cellular read-out.

\subsubsection{Induction and regulation of the $\mathrm{CPC} / \mathrm{GC}$}

The regulatory gene of the yeast General Control was named GCN4 (general control nonderepressible), and its mRNA contains four small coding sequences, which are part of an unusually long leader region preceding the actual coding sequence. The Aspergillus gene homologous to GCN4 is $c p c A$. The $c p c A$ gene of $A$. fumigatus has a size of about 2.2 $\mathrm{kb}$, from which the coding sequence spans $810 \mathrm{bp}$. Two small ORFs exist preceding the cpcA coding region, which are similar to the four regulatory uORFS of GCN4. Structure and function of the GCN4 gene product as well as the interplay between the two has been studied intensively. The C-terminal part contains a leucine-zipper motif that is important for the dimerisation of the protein. In addition the $\mathrm{C}$-terminal domain comprises a conserved DNA binding region, which is important for transcriptional regulation of Gcn4p target genes (Ellenberger et al., 1992). The N-terminal region is divided in two parts that represent different transcriptional activation domains (Drysdale et al., 1998). Regulation of cpcA expression in A. nidulans is mediated on the transcriptional as well as the translational level (Hoffmann et al., 2001).

In S. cerevisiae expression of GCN4 is primarily regulated on transcriptional level, whereas in C. albicans transcription of GCN4 is more important than translation (Tournu et $a l ., 2005)$. In detail, translational regulation of gene expression via the upstream open reading frames of $G C N 4 / c p c A$ transcripts acts like follows: under sated conditions, expression levels are low at a basal level due to the fact that scanning ribosomes initiate translation on the $G C N 4 / c p c A$ transcript at the first uORF. Important for this are the amounts of so-called tertiary complexes, which consist of the translation initiator eIF2, GTP and a tRNAMet, and these three components are crucial factors for initiation of translation. The tertiary complex enables the ribosomes to bind at the first uORF within the leader sequence of $G C N 4 / c p c A$. After reaching the stop codon, ribosomes disassemble into the two ribosomal sub-domains. To initiate another round of translation, a new tertiary complex has to be assembled together with the small ribosomal subunit. In case 
concentrations of tertiary complexes are high, one of the downstream uORFs will be translated. As the distance between the stop codon of the distal uORF and the start codon of the actual coding sequence is too close translational re-initiation is hampered and almost no gene product is expressed.

Under starvation conditions expression is drastically de-repressed. In case of amino acid starvation, uncharged tRNA molecules accumulate and bind to a sensor kinase called Gcn2p or $\mathrm{CpcC}$, respectively. The activated kinase phosphorylates a subunit of the initiation factor eIF2, resulting in diminished levels of tertiary complexes and therefore lower rates of translation initiation. In consequence, overall cellular translation is downregulated but translation initiation at the $c p c A / G C N 4$ coding region increases, as competent ribosomes that have failed to translated the distal UORF are now able to bind at the start codon of the coding sequence to initiate the translation (Hoffmann et al., 2001; Braus et al., 2003; Krappmann et al., 2004).

Regulation of Aspergillus CpcA expression on the transcriptional level has been investigated in detail for A. nidulans (Hoffmann et al., 2001). There, synthesized CpcA binds to so called CPREs (Cross-Pathway Control Recognition Elements) that locate within the promoter region of target genes. It could be shown that two of these CPREs are part of the promoter region of $c p c A$ suggesting a functional auto-regulatory loop (Hoffmann et al., 2001). A regulation like this has not been proven for CpcA expression in A. fumigates, however, it was shown that two highly conserved CPREs are also present within the $c p c A$ promoter region (Krappmann et al., 2004). The proximal one (5'ATGACTCAC-3', pos. -1062 till -1053) is identical to its A. nidulans counterpart, the distal one shows a difference in one site of the sequence in comparison to the A. nidulans CPRE (5'-ATGACTCgAC-3', -1273 till -1264). This leads to the suggestion that binding of CpcA to this recognition site might be weaker (Hinnebusch, 1984; Thireos et al., 1984; Arndt and Fink, 1986).

In addition, a variety of other factors that influence activity of the Cross-Pathway Control/General Control activators CpcA/Gcn4p were identified, among them: glucose, nitrogen and purine starvation (Mösch et al., 1991; Grundmann et al., 2001; Hinnebusch and Natarajan, 2002; Braus et al., 2003).

In Verticillium species so far the influence of Cross-Pathway Control on development and pathogenicity of the fungus has only been slightly investigated. Singh et al. (2010) could show in plant experiments that $C P C l$, the activator of cross pathway control, is upregulated, when the fungus is growing inside the plant (Singh et al., 2010). During 
infection and colonization of its host $V$. longisporum activates the cross pathway control to cope with an imbalanced amino acid supply (Singh et al., 2010).

\subsection{Aim of this work}

The aim of this work was to investigate how the fungus adapts within the plant xylem sap and what regulatory genes might be involved. Xylem sap is the major source of nutrient supply for Verticillium when colonizing its host.

Earlier studies of Singh et al. (2010) revealed the amount of amino acids avaible for the fungus. Because these amounts are limited the fungus needs to deal with an imbalanced amino acid supply. In a first approach we wanted to see if the regulator of cross pathway control $C P C 1$ is required for successful infection and colonization of its host. For the survival of the fungus the acquisition of amino acids is necessary to cope with the conditions given by the host plant. Imbalanced amino acid supply requires $C P C 1$. To further analyze the influence of $C P C l$ in pathogenicity mutants silenced in $C P C l$ for $V$. longisporum and mutants with a knockout of $C P C l$ for $V$. dahliae were generated. One aim of this work was to generate knockdowns or knockouts of this gene in $V$. longisporum and $V$. dahliae, than to investigate the influence of analogues on these mutants ex planta, and later observe the mutant in planta. For these mutants assays in planta were performed to observe the propagation and infection of mutants in the plant.

For infection and colonization secreted proteins and secondary metabolites play a crucial role for the survival of the fungus in the host plant. They are also known to be important for pathogenicity of the fungus. These proteins or compounds might be useful to adapt to the plants pathogen reaction or either might be necessary to counteract the pathogen response of the host. The key regulator of secondary metabolism is LAE1. To analyze the influence of $L A E 1$ in pathogenicity mutants silenced as well as knockouts of LAE1 were generated. These mutants were further characterized by assays in planta to observe the propagation and infection of mutants in the plant.

Successful infection and colonization of the host requires proteins or enzymes that are capable of acquiring nutrition for the fungal pathogen, or provide opportunities of breaking the plant cell wall. Secreted proteins and secondary metabolites are the key players for these reactions. These proteins or enzymes might be useful to adapt to the plants pathogen reaction or either might be important to counteract the pathogen response of the host plant. Among these enzymes, lipases and cutinases, with their potential roles in the hydrolyzation of the plant cuticle and surface waxes, may contribute to pathogen infection at an early 
stage, when the fungus first comes into contact with its host.A secreted lipase has been isolated from a fungal culture treated with xylem sap. As an example for secreted proteins a lipase was characterized as well. This triacylglycerol lipase (TAGL) might be required for breaking the plant cell well and enter the host. Silenced mutants of TAGL were generated and analysed in plant infection assays. 


\section{Chapter 2. Materials and Methods}

\subsection{Microbial strains and growth conditions}

\subsubsection{Bacterial strains}

Escherichia coli strains DH5 $\alpha$, ccdB-resistant strain (Invitrogen, Karlsruhe, Germany) and Agrobacterium tumefaciens AGL1 were used for transformation procedures in this work. The E. coli strain was cultivated at $37^{\circ} \mathrm{C}$, while the A. tumefaciens strain was grown at $25-28^{\circ} \mathrm{C}$. Both organisms were grown in Luria Bertani (LB) medium $(0.5 \%$ yeast extract, $1 \%$ tryptone, $0.5 \% \mathrm{NaCl}$ ).

\subsubsection{Verticillium strains}

Eight isolates of Verticillium species from different hosts were used in this study (Table 1). Most of the Verticillium isolates were kindly provided by Prof. Andreas von Tiedemann, Georg-August University Göttingen (according to Zeise and Tiedemann, 2001; 2002a) except the Vl-Bob70 isolate was derived from VdBob70 isolate (Qin et al., 2006), Vertcillium dahliae VdJR2 (Fradin et al., 2009), was provided by Bart Thomma from the Laboratory of Phytopathology in Wageningen (The Netherlands). All strains were inoculated in potatoe dextrose broth (PDB) (Sigma-Aldrich Chemie GmbH, Munich, Germany), Minimal medium (MM) (Bennett \& Lasure, 1991), Czapek-Dox medium (CDM) (Smith, 1960) or simulated xylem medium (SXM) (Neumann and Dobinson, 2003) and incubated for 7-10 days at $25^{\circ} \mathrm{C}$.

\subsubsection{Spore preparation}

The fungal isolates were grown separately in Czapek-Dox liquid medium for 10 days, at $25^{\circ} \mathrm{C}$ on a shaker, $120 \mathrm{rpm}$. Fungal conidia were harvested by filtering the culture through miracloth membrane (Calbiochem, Darmstadt, Germany), the filtrate was centrifuged at $5,000 \mathrm{rpm}$ for 20 minutes at $4^{\circ} \mathrm{C}$. After a washing step with sterile tap water, the sediment was resuspended in sterile tap water. The number of spores was counted under microscope using a counting chamber and spore density was adjusted to $10^{7} \mathrm{spores} / \mathrm{ml}$. Glycerin was added to the spore suspension at the final concentration of $20 \%$ and aliquots of the spore suspension were frozen in liquid nitrogen and stored at $-80^{\circ} \mathrm{C}$. 
Table 1. Verticillium isolates used in this study.

\begin{tabular}{|c|c|c|c|c|}
\hline Isolate & Species & Host & Geographic origin & Reference \\
\hline $\begin{array}{ll}\mathrm{Vd}-73 \\
\end{array}$ & V. dahliae & Linum usitatissimum (linseed) & Mecklenburg/Germany & $\begin{array}{l}\text { Zeise and von } \\
\text { Tiedermann, } 2001\end{array}$ \\
\hline Vd-JR2 & V. dahliae & Solanum lycopersicum & Canada & Fradin et al., 2009 \\
\hline Vl-40 & V. longisporum & Brassica napus (rapeseed) & Mecklenburg/Germany & $\begin{array}{l}\text { Zeise and von } \\
\text { Tiedermann, 2002a }\end{array}$ \\
\hline Vl-43 & V. longisporum & Brassica napus (rapeseed) & Mecklenburg/Germany & $\begin{array}{l}\text { Zeise and von } \\
\text { Tiedermann, 2002a }\end{array}$ \\
\hline $\begin{array}{l}\text { Vl- } \\
\text { Bob70 }\end{array}$ & V. longisporum & $\begin{array}{l}\text { Brassica oleracea var. botrytis } \\
\text { (cauliflower) }\end{array}$ & California/USA & Qin et al. 2009 \\
\hline$\overline{\mathrm{Va}-1}$ & V. albo-atrum & Solanum tuberosum (potato) & Wiconsin/USA & $\begin{array}{l}\text { Zeise and von } \\
\text { Tiedermann, 2002b }\end{array}$ \\
\hline
\end{tabular}

Table 2. The plasmids used in this study.

\begin{tabular}{|c|c|c|c|}
\hline Name & Type & Description & Source \\
\hline pDONR/Zeo & Gateway & Containing a gateway cassette, Zeocin ${ }^{\mathrm{R}}$ & Invitrogen \\
\hline pJET1.2 & Cloning & A dephosphorylated cloning vector with blunt ends & Fermentas \\
\hline pKO2 & Cloning & Vector for gene disruption & Tran 2011 \\
\hline pGS1 & Cloning & Vector for gene silencing & Tran 2011 \\
\hline $\mathrm{pKOCPC1}$ & Cloning & Vector for deletion of $C P C 1$ & Timpner et al. 2013 \\
\hline pKOLAE1 & Cloning & Vector for deletion of $L A E 1$ & This study \\
\hline pGSCPCl & Cloning & Vector for silencing of $C P C 1$ & This study \\
\hline pGSLAE1 & Cloning & Vector for silencing of $L A E 1$ & This study \\
\hline pGSTAGL1 & Cloning & Vector for silencing of triacyl glycerol lipase & This study \\
\hline
\end{tabular}




\subsection{Bioinformatic methods}

\subsubsection{Primer designing}

The primers used in this study (Table 3) were designed using the Primer3 program (http://frodo.wi.mit.edu/primer3/ (Rozen and Skaletsky, 2000)) and purchased from Invitrogen (Karlsruhe, Germany) or from Eurofins-MWG (Ebersberg, Germany), the amplification efficiency of each primer pair was checked with Mastercycler Gradient (Eppendorf, Hamburg, Germany).

Table 3 Primers used in this study (Underlined part of the sequence represent cutting sites)

\begin{tabular}{|c|c|c|c|}
\hline Primer & Size & Sequence (5'-3') & Reference \\
\hline $\begin{array}{l}\text { OLG } 70 \\
\text { OLG } 71\end{array}$ & $\begin{array}{l}20 \mathrm{mer} \\
19 \mathrm{mer}\end{array}$ & $\begin{array}{l}\text { CAG CGA AAC GCG ATA TGT AG } \\
\text { GGC TTG TAG GGG GTT TAG A }\end{array}$ & $\begin{array}{l}\text { Eynck et al. } \\
2007\end{array}$ \\
\hline $\begin{array}{l}\text { H2aRTleft } \\
\text { H2aRTright }\end{array}$ & $\begin{array}{l}22 \mathrm{mer} \\
22 \mathrm{mer}\end{array}$ & $\begin{array}{l}\text { CCC GTG ACA ACA AGA AGA CTC G } \\
\text { GCA GGA AAG AAA AGC CAA AAC C }\end{array}$ & $\begin{array}{l}\text { Singh et al. } \\
2011\end{array}$ \\
\hline $\begin{array}{l}\text { Cpc1RTleft } \\
\text { Cpc1RTright }\end{array}$ & $\begin{array}{l}22 \mathrm{mer} \\
22 \mathrm{mer}\end{array}$ & $\begin{array}{l}\text { CAG CCT ACA CAT CCC AAC AAC C } \\
\text { TCC GTT TCC AGC AGC AGA TAG T }\end{array}$ & This study \\
\hline $\begin{array}{l}\text { Cpclgateleft } \\
\text { Cpclgateright }\end{array}$ & $\begin{array}{l}50 \mathrm{mer} \\
49 \mathrm{mer}\end{array}$ & $\begin{array}{l}\text { GGG GAC AAG TTT GTA CAA AAA AGC AGG } \\
\text { CTT CAT GAA CAT CGC GGA TTT CG } \\
\text { GGG GAC CAC TTT GTA CAA GAA AGC TGG } \\
\text { GTC CTG CCA GAG TGT TGC GAG C }\end{array}$ & This study \\
\hline $\begin{array}{l}\text { F1CpcAlc } \\
(\text { PacI })\end{array}$ & 33 mer & $\begin{array}{l}\text { GGG TTA ATT AAG GCT ATC GAG CAT ACG } \\
\text { AAG AAC }\end{array}$ & This study \\
\hline $\begin{array}{l}\text { F1CpcArc } \\
(\text { SacI })\end{array}$ & $31 \mathrm{mer}$ & $\begin{array}{l}\text { GGG GAG CTC CCA TCC ATA TAT TCT GGC } \\
\text { GAT T }\end{array}$ & This study \\
\hline $\begin{array}{l}\text { ProbeCpc1left } \\
\text { ProbeCpc1right }\end{array}$ & $\begin{array}{l}20 \mathrm{mer} \\
20 \mathrm{mer}\end{array}$ & $\begin{array}{l}\text { CGG CCC TCA AAA ATT GTT CC } \\
\text { GAC TTA AAT TGA GAT ATC CG }\end{array}$ & This study \\
\hline $\begin{array}{l}\text { Cpc1Seqleft } \\
\text { Cpc1Seqright }\end{array}$ & $\begin{array}{l}19 \mathrm{mer} \\
21 \mathrm{mer}\end{array}$ & $\begin{array}{l}\text { ATG GCT TCC CTC CAG TTC C } \\
\text { CTA GTC GCT GGT TGA CTG ACC }\end{array}$ & This study \\
\hline $\begin{array}{l}\text { RTCpc1left } \\
\text { RTCpc1right }\end{array}$ & $\begin{array}{l}22 \mathrm{mer} \\
22 \mathrm{mer}\end{array}$ & $\begin{array}{l}\text { CAG CCT ACA CAT CCC AAC AAC C } \\
\text { TCC GTT TCC AGC AGC AGA TAG T }\end{array}$ & This study \\
\hline $\begin{array}{l}\text { VDLaeF2r } \\
\text { VDLaeF21 }\end{array}$ & $\begin{array}{l}22 \mathrm{mer} \\
22 \mathrm{mer}\end{array}$ & $\begin{array}{l}\text { GAA TAC ATC CTG GTA GCC TTC G } \\
\text { CTG GAC AGG AAG TAC AAC GAC A }\end{array}$ & This study \\
\hline
\end{tabular}




\begin{tabular}{|c|c|c|c|}
\hline $\begin{array}{l}\text { VDLaeF2Lc } \\
\text { (PstI) } \\
\text { VDLaeF2Rc } \\
\text { (BamHI) }\end{array}$ & $\begin{array}{l}29 \text { mer } \\
30 \mathrm{mer}\end{array}$ & $\begin{array}{l}\text { GGG CTG CAG AGA CCA GAC GCC CCT AAA } \\
\text { CT } \\
\text { GGG GGA TCC CCT GGA CAG GAA GTA CAA } \\
\text { CGA }\end{array}$ & This study \\
\hline $\begin{array}{l}\text { VDLaeF11 } \\
\text { VDLaeF1r }\end{array}$ & $\begin{array}{l}20 \mathrm{mer} \\
20 \mathrm{mer}\end{array}$ & $\begin{array}{l}\text { GTG CAG CAG GTA CTG GCT TT } \\
\text { TGA TAG CTG ACA CGC GAA AC }\end{array}$ & This study \\
\hline $\begin{array}{l}\text { VDLaeF1Lc } \\
\text { (EcoRI) } \\
\text { VDLaeF1Rc } \\
\text { (EcoRV) }\end{array}$ & $\begin{array}{l}29 \text { mer } \\
29 \text { mer }\end{array}$ & $\begin{array}{l}\text { GGG GAA TTC GTG CAG CAG GTA CTG GCT } \\
\text { TT } \\
\text { GGG GAT ATC TGA TAG CTG ACA CGC GAA } \\
\text { AC }\end{array}$ & This study \\
\hline $\begin{array}{l}\text { KOCpcF1Lc } \\
\text { (PacI) } \\
\text { KOCpcF1Rc } \\
\text { (SacI) }\end{array}$ & $\begin{array}{l}31 \text { mer } \\
29 \text { mer }\end{array}$ & 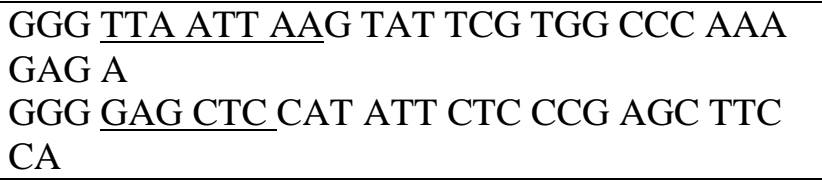 & This study \\
\hline $\begin{array}{l}\text { RTCpcAleft } \\
\text { RTCpcAright }\end{array}$ & $\begin{array}{l}22 \mathrm{mer} \\
22 \mathrm{mer}\end{array}$ & $\begin{array}{l}\text { CAG CCT ACA CAT CCC AAC AAC C } \\
\text { TCC GTT TCC AGC AGC AGA TAG T }\end{array}$ & This study \\
\hline $\begin{array}{l}\text { LaeART1left } \\
\text { LaeART1right }\end{array}$ & $\begin{array}{l}22 \mathrm{mer} \\
22 \mathrm{mer}\end{array}$ & $\begin{array}{l}\text { AAA CCG CAC TAC AGA CCA GAC G } \\
\text { GAA GCG AAG GCT ACC AGG ATG T }\end{array}$ & This study \\
\hline $\begin{array}{l}\text { LipRT1left } \\
\text { LipRT1right }\end{array}$ & $\begin{array}{l}22 \mathrm{mer} \\
22 \mathrm{mer}\end{array}$ & $\begin{array}{l}\text { ACA TTT TGT CAC GAG GGG GAT T } \\
\text { TAC CAC GCT CTT CTT GCT GCT C }\end{array}$ & This study \\
\hline $\begin{array}{l}\text { ProbeLipleft } \\
\text { ProbeLipright }\end{array}$ & $\begin{array}{l}22 \mathrm{mer} \\
21 \mathrm{mer}\end{array}$ & $\begin{array}{l}\text { TTT CCA GCA AGC TGT ATT CTC A } \\
\text { CGA TGG TTA ATT GGC TGT CAT }\end{array}$ & This study \\
\hline $\begin{array}{l}\text { ProbeLae1left } \\
\text { ProbeLae1right }\end{array}$ & $\begin{array}{l}22 \mathrm{mer} \\
22 \mathrm{mer}\end{array}$ & $\begin{array}{l}\text { CGA GAA CAG CAT TGA TTA CGT C } \\
\text { TTT CAA TCT CTT CAG TCG TCC A }\end{array}$ & This study \\
\hline $\begin{array}{l}\text { VdCPC1KOPro } \\
\text { be-L } \\
\text { VdCPC1KOPro } \\
\text { be-R }\end{array}$ & $\begin{array}{l}20 \mathrm{mer} \\
20 \mathrm{mer}\end{array}$ & $\begin{array}{l}\text { TCC CAA CAA CCT GCC TAA AC } \\
\text { AGA GTC CAG AAC AGC ACG GT }\end{array}$ & This study \\
\hline $\begin{array}{l}\text { VdLAE1KOPro } \\
\text { be-L } \\
\text { VdLAE1KOPro } \\
\text { be-R }\end{array}$ & $\begin{array}{l}20 \mathrm{mer} \\
20 \mathrm{mer}\end{array}$ & $\begin{array}{l}\text { TCC CAA CAA CCT GCC TAA AC } \\
\text { AGA GTC CAG AAC AGC ACG GT }\end{array}$ & This study \\
\hline
\end{tabular}

\subsubsection{Tools for DNA sequence analysis}

DNA sequences were verified by using the 4Peaks software (www.mekentosj.com) For molecular analyses, DNA sequences were collected from the Verticillium group database (http://www.broadinstitute.org/annotation/genome/verticillium_dahliae/MultiHome.html). The open reading frame (ORF) of each gene was identified and translated into a protein sequence by using the ORF Finder program (http://www.ncbi.nlm.nih.gov/projects/gorf/). The protein sequence was for conserved domains or motifs using InterProScan (http://www.ebi.ac.uk/Tools/InterProScan/) or Pfam (http://pfam.sanger.ac.uk/) or Prosite 
(http://expasy.org/prosite/). The information of DNA sequences was determined by blasting the sequences in Genbank (http://blast.ncbi.nlm.nih.gov/). Restriction sites of the DNA sequences were determined by using NEBcutter V2.0 program from New England Biolabs (http://tools.neb.com/NEBcutter2/).

\subsubsection{DNA analysis and comparison}

Characterization of CPC1 and LAE1 was based on sequences derived from the Verticillium group database (http://www.broadinstitute.org/annotation/genome/verticillium_dahliae/ MultiHome.html). The introns and exons were determined by comparing the cDNA sequences with their genomic DNA sequences using the ClustalW program (Thompson et al., 1994). For DNA analysis and comparison in more details, the commercial software Geneious Pro 5.0.4 (Biomatter Ltd, Auckland, New Zealand) was used.

\subsubsection{Phylogenetic analysis}

The phylogenetic trees were constructed with the MEGA 5.0 software (Tamura et al., 2007) based on the neighbor-joining method (Saitou and Nei, 1987). The statistical reliabilities of the internal branches were assessed for all trees by using the bootstraps of 1,000 replicates.

\subsubsection{Drawing of plasmid maps and models}

The plasmid maps and models in this study were drawn using the Savvy program (http://www.bioinformatics.org/savvy/) and Adobe Photoshop software.

\subsection{Genetic manipulations}

\subsubsection{E. coli transformation}

\subsubsection{Preparation of $E$. coli competent cells}

10 fresh colonies of E. coli were inoculated into $250 \mathrm{ml}$ of SOB medium (2\% tryptone, $0.5 \%$ yeast extract, $10 \mathrm{mM} \mathrm{NaCl}, 2.5 \mathrm{mM} \mathrm{KCl}, 10 \mathrm{mM} \mathrm{MgCl}_{2}, 10 \mathrm{mM} \mathrm{MgSO}_{4}$ ) in a 1 liter flask. The flask was incubated on a shaker $(100 \mathrm{rpm})$ at $20^{\circ} \mathrm{C}$ until the $\mathrm{OD}_{600}$ of the culture was about $0.6-0.8$. The culture was kept in ice for $10 \mathrm{~min}$ and centrifuged at 5,000 rpm for $10 \mathrm{~min}$ at $4^{\circ} \mathrm{C}$. The sediment was then resuspended in $80 \mathrm{ml}$ TB buffer $(10 \mathrm{mM}$ PIPES/HEPES, $\left.15 \mathrm{mM} \mathrm{CaCl}_{2} \cdot 2 \mathrm{H}_{2} \mathrm{O}, 250 \mathrm{mM} \mathrm{KCl} ; 55 \mathrm{mM} \mathrm{MnCl}_{2} ; \mathrm{pH} 6.7\right)$. This cell suspension was incubated on ice for $10 \mathrm{~min}$ followed by centrifugation at $4,000 \mathrm{rpm}$ for $10 \mathrm{~min}$ at $4^{\circ} \mathrm{C}$. The sediment was resuspended in $20 \mathrm{ml} \mathrm{TB}$ followed by the addition of DMSO by gently swirling to a final concentration of $7 \%$. This cell suspension was further 
incubated for $10 \mathrm{~min}$ on ice and dispensed $(400 \mu \mathrm{l})$ into aliquots. The aliquots were frozen in liquid nitrogen and stored at $-80^{\circ} \mathrm{C}$.

\subsubsection{2. $E$. coli transformation}

An aliquot of competent cells was first thawed on ice. About 5-10 $\mu$ of the ligation reaction or $50 \mathrm{ng}$ of a plasmid was added to $100 \mu \mathrm{l}$ of the competent cells and incubated for $20 \mathrm{~min}$ on ice. The cells were then subjected to heat shock at $42^{\circ} \mathrm{C}$ for $40 \mathrm{sec}$ and then kept on ice for $5 \mathrm{~min}$. About $800 \mu \mathrm{l}$ of SOC medium (SOB $+20 \mathrm{mM}$ glucose) was added to the mixture followed by a recovery at $37^{\circ} \mathrm{C}$ with shaking at $180 \mathrm{rpm}$ for $1 \mathrm{~h}$. The cells were collected by centrifugation at $10,000 \mathrm{rpm}$ for $1 \mathrm{~min}$ and plated on a LB agar plate supplemented with an appropreate antibiotic for selection (the final concentration of ampicillin or kanamycin is $100 \mu \mathrm{g} / \mathrm{ml}$ and $30 \mu \mathrm{g} / \mathrm{ml}$ for zeocin).

\subsubsection{Agrobaterium tumefaciens transformation}

\subsubsection{Preparation of competent cells}

A. tumefaciens strain AGL1 was used for the mediated transformation of Verticillium species. To prepare competent cells, a single colony of this bacterium was grown in $50 \mathrm{ml}$ of $\mathrm{LB}$ medium with $50 \mu \mathrm{g} / \mathrm{ml}$ carbenicillin at $28^{\circ} \mathrm{C}, 160 \mathrm{rpm}$ for overnight. The culture $\left(\mathrm{OD}_{600}=0.8\right)$ was cooled on ice for $15 \mathrm{~min}$ and the cells were collected by centrifugation at 5,000 rpm for $10 \mathrm{~min}$ at $4^{\circ} \mathrm{C}$. The sediment was resuspended in $20 \mathrm{ml}$ of sterile ice-cold $100 \mathrm{mM} \mathrm{MgCl} 2$ solution, and kept on ice for $1 \mathrm{~h}$. The cells were harvested by centrifugation as above, then resuspended in $20 \mathrm{ml}$ of sterile ice-cold $20 \mathrm{mM} \mathrm{CaCl}_{2}$ solution and incubated on ice for 4-5 h to obtain competent cell suspension. Glycerol was added to a final concentration of $20 \%$ and aliquots of $200 \mu \mathrm{l}$ were frozen in liquid nitrogen and stored at $-80^{\circ} \mathrm{C}$.

\subsubsection{Agrobacterium transformation}

The freeze-thaw method (Jyothishwaran et al., 2007) was used for A. tumefaciens transformation. A tube of frozen competent cells $(200 \mu \mathrm{l})$ was kept on ice for $10 \mathrm{~min}$ to thaw and $10 \mu \mathrm{l}$ of a binary vector $(100 \mathrm{ng} / \mu \mathrm{l})$ was mixed to the competent cells. The tube was inverted gently for three times and incubated on ice for $5 \mathrm{~min}$, then frozen in liquid nitrogen for $10 \mathrm{~min}$ and thawed at $37^{\circ} \mathrm{C}$ in a heat block for $5 \mathrm{~min}$. The mixture was added $800 \mu \mathrm{l}$ of SOC medium without antibiotics and incubated at $28^{\circ} \mathrm{C}$ for $1 \mathrm{~h}$ in a shaker at $160 \mathrm{rpm}$. The cells collected by centrifugation at 5,000 rpm for 5 min were spread on a LB plate containing $100 \mu \mathrm{g} / \mathrm{ml}$ kanamycin and incubated at $25-28^{\circ} \mathrm{C}$ or at room temperature 
for 48-72 hours to gain colonies. Colony PCR was employed to screen positive colonies using a specific primer pair.

\subsubsection{Agrobacterium-mediated Verticillium transformation}

For A. tumefaciens-mediated transformation (ATMT) of Verticillium species, a plasmid carrying bacterial colony was grown $5 \mathrm{ml}$ of LB medium supplemented with $100 \mu \mathrm{g} / \mathrm{ml}$ kanamycin (also with $50 \mu \mathrm{g} / \mathrm{ml}$ carbenicillin to avoid contamination of other bacteria if necessary) for overnight. About $0.5-1.0 \mathrm{ml}$ of the culture was diluted with induction medium (IM) (5 mM glucose, 0,5\% glycerol, $40 \mathrm{mM}$ MES, 1x Salt solution) containing $200 \mu \mathrm{M}$ acetosyringone (AS) to get an optical density of 0.2 at the wavelength at $600 \mathrm{~nm}$ $\left(\mathrm{OD}_{600}=0.2\right)$, then the diluted culture was grown at $28^{\circ} \mathrm{C}$ for $4-5 \mathrm{~h}$ at $160 \mathrm{rpm}$. A mixture of equal volumes $(150 \mu \mathrm{l})$ of the bacterial culture and the fresh spore suspension $\left(10^{6} \mathrm{spores} / \mathrm{ml}\right)$ was spread onto the filter paper of $85-\mathrm{mm}$ diameter (Satorius, Göttingen, Germany) on a agar plate of induction medium containing $200 \mu \mathrm{M}$ acetosyringone (IMAS) that is identical to liquid IM, except it contains $5 \mathrm{mM}$ of glucose instead of $10 \mathrm{mM}$ glucose. Following co-cultivation at $25^{\circ} \mathrm{C}$ for $48-72 \mathrm{~h}$, the filter paper was transferred to a PDA (potato dextrose agar) plate containing hygromycin B $(50 \mu \mathrm{g} / \mathrm{ml})$ or nourseothricin $(50 \mu \mathrm{g} / \mathrm{ml})$ as the selection agent for fungal transformants, and cefotaxime $(50 \mu \mathrm{g} / \mathrm{ml})$ to kill the A. tumefaciens cells. The plates were sealed with parafilm, inverted and incubated at $25^{\circ} \mathrm{C}$ for $8-10 \mathrm{~d}$.

\subsubsection{Plasmid isolation from $E$. coli}

The plasmids carrying the gateway cassette(s) such as, pDONR/Zeo and pGS1, were maintained and propagated in the $c c d$ B-resistant E. coli strain (Invitrogen, Karlsruhe, Germany). For other plasmids, the E. coli strain DH5 $\alpha$ was used instead. A single colony containing a plasmid of interest was inoculated in $5 \mathrm{ml}$ of LB liquid medium with an appropriate antibiotic (100 $\mu \mathrm{g} / \mathrm{ml}$ for ampicillin and kanamycin, $30 \mu \mathrm{g} / \mathrm{ml}$ for zeocin) and incubated at $37^{\circ} \mathrm{C}, 180 \mathrm{rpm}$ for overnight. The plasmid was isolated using QIAprep Spin Miniprep Kit (Qiagen, Hilden, Germany) according to the manufacturer's instructions.

\subsubsection{Isolation of nucleic acids from Verticillium}

Fungal isolates were grown in potato dextrose broth (PDB) (Sigma-Aldrich Chemie $\mathrm{GmbH}$, Munich, Germany) or in liquid simulated xylem medium (SXM) (Neumann and Dobinson, 2003) for one week, at $25^{\circ} \mathrm{C}$ with shaking at $100 \mathrm{rpm}$. The fungal mycelium was harvested with miracloth (Calbiochem, Darmstadt, Germany) and ground to fine powder in 
liquid nitrogen using a pestle and mortar. The fungal powder was used directly for nucleic acid extraction or frozen in liquid nitrogen and preserved at $-80^{\circ} \mathrm{C}$.

Genomic DNA was extracted from the fungal powder according to Kolar et al., 1988 with some modifications. About $800 \mu \mathrm{g}$ of the fungal powder was transferred to a $2.0 \mathrm{ml}$ tube $800 \mu \mathrm{l}$ of fresh lysis buffer (50 mM Tris-HCl, pH7.2; $50 \mathrm{mM}$ EDTA; 3\% SDS; $1 \%$ 2-Mercaptoethanol) was added to the tube. The tube was vortexed at maximum speed for 10 seconds and incubated at $65^{\circ} \mathrm{C}$ for $1 \mathrm{~h}$. About $800 \mu \mathrm{l}$ of phenol, chloroform and isoamyl alcohol mixture (25:24:1) was added to the tube and the tube was inverted for several times. The tube was centrifuged at $13,000 \mathrm{rpm}$ for $15 \mathrm{~min}$ and $400-500 \mu \mathrm{l}$ of the supernatant was transferred carefully to a new $2 \mathrm{ml}$ tube. $800 \mu \mathrm{l}$ of phenol, chloroform, isoamyl alcohol mixture was added to the supernatant and the tube was centrifuged again as discribed above. The supernatant was transferred to a new tube containing $600 \mu 1$ isopropanol and $25 \mu \mathrm{l}$ of $3 \mathrm{M} \mathrm{NaAcOH}$. The tube was centrifuged at $13000 \mathrm{rpm}$ for $2 \mathrm{~min}$. The sediment was washed twice with $1 \mathrm{ml}$ of $70 \%$ ethanol by centrifugation. The sediment was dried at $37^{\circ} \mathrm{C}$ for $20 \mathrm{~min}$ and dissolved in $\mathrm{H}_{2} \mathrm{O}$. The DNA sample was treated with $4 \mu \mathrm{l}$ of RNase A at $37^{\circ} \mathrm{C}$ for 30 min to eliminate RNA. DNA concentration was measured using NanoDrop ND-1000 spectrophotometer (PEQLAB, Erlangen, Germany). The DNA quality was checked on $1 \%$ agarose gel using $5 \mu$ l genomic DNA.

Total fungal RNA was extracted by using the RNeasy Plant Mini Kit (Qiagen, Hilden, Germany) or Trizol reagent (Invitrogen, Karlsruhe, Germany) according to the manufacturer's instructions.

\subsubsection{PCR and DNA purification}

Polymerase chain reaction (PCR) amplifications was performed in $25-\mu 1$ volumes with the PCR cycler (MWG-Biotech Primus, Ebersberg, Germany). For analysis or confirmation purposes, Taq polymerase (Fermentas, St. Leon-Rot, Germany) was used. PCR conditions including an initial denaturation at $94^{\circ} \mathrm{C}$ for $3 \mathrm{~min}$ followed by $30-35$ cycles of denaturation at $94^{\circ} \mathrm{C}$ for $1 \mathrm{~min}$, annealing at $55-60^{\circ} \mathrm{C}$ for $40 \mathrm{~s}$ and extension at $72^{\circ} \mathrm{C}$ for $1 \mathrm{~min} / \mathrm{kb}$; a final extension at $72^{\circ} \mathrm{C}$ for $10 \mathrm{~min}$ and storage at $4-8^{\circ} \mathrm{C}$ until used.

For cloning purposes, the high-fidelity Phusion DNA polymerase (Finnzymes, Espoo, Finland) was used with the constitutions and conditions for PCR based on the manufacturer's instructions. The PCR products were analyzed on a 1\% agarose gel. The DNA fragments were excised and purified with QIAquick Gel Extraction Kit (Qiagen, 
Hilden, Germany). The purified DNA fragments were used for direct sequencing or for cloning.

\subsubsection{Cloning and sequencing}

For digestion of DNA fragments or plasmids $250 \mathrm{ng}$ of a PCR product and $500 \mathrm{ng}$ of a plasmid were digested with appropriate restriction enzymes (Fermentas, St. Leon-Rot, Germany) for $2 \mathrm{~h}$. The restricted DNA samples were analyzed on a $1 \%$ agarose gel and the expected bands were purified using the QIAquick Gel Extraction Kit (Qiagen, Hilden, Germany) according to the provided manual. For ligation, about $150 \mathrm{ng}$ of the restricted insert DNA was mixed with $50 \mathrm{ng}$ of the restricted plasmid (3:1) along with $1 \mu \mathrm{l}$ of T4 DNA ligase (Fermentas, St. Leon-Rot, Germany) and 1X T4 DNA ligase buffer in a total volume of 10-20 $\mu \mathrm{l}$. The ligation reaction was incubated at room temperature for $1 \mathrm{~h}$ or at $16^{\circ} \mathrm{C}$ in a heating block for overnight. The ligation mixture was used to transform E. coli competent cells.

For sequencing of the target genes, the PCR product of each gene amplified by Phusion DNA polymerase was purified and cloned directly into the pJET1.2/blunt cloning vector using the CloneJET ${ }^{\mathrm{TM}}$ PCR Cloning Kit (Fermentas, St. Leon-Rot, Germany) according to the manufacturer's instructions. The whole ligation mixture was transformed into E. coli DH5 $\alpha$ competent cells. Colony PCR was employed to screen positive colonies from each cloning procedure. At least, 10-15 positive clones were selected and grown in LB (LuriaBertani) liquid medium added $100 \mu \mathrm{g} / \mathrm{ml}$ of ampicillin, recombinant plasmids were isolated using the QIAprep Spin Miniprep Kit (Qiagen, Hilden, Germany). The sequencing of the recombinant plasmids was performed with specific primers by Göttingen Genomics Laboratory (G2L), Georg-August University of Göttingen, Germany.

\subsection{Generation of vector for gene silencing in $V$. longisporum}

To investigate the role in the survival of $V$. longisporum when it inhabits the xylem vessel of the host plant, we decided to knock-down the isogenes, VlCPCl-1 and VlCPCl-2 by RNA silencing because the conventional knock-out was not practical due to the presence of two isogenes. RNA mediated gene silencing acts in a sequence-specific manner and should silence both the isogenes. It has been successfully implemented in several fungi for targeted gene silencing (Nakayashiki, 2005). Silencing, particularly, by hairpin RNA was found to be most efficient in the filamentous fungus, M. oryzae (Kadotani et al., 2003). In previous works (Singh et al. 2010, Singh et al. 2012) silencing of certain V. longisporum 
genes was performed. Therefore, a hairpin double-stranded RNA mediated gene silencing approach was applied for RNA silencing of VlCPCl-1 and VlCPCl-2 in V. longisporum.

To generate silencing plasmid a 544 bp fragment of $V l C P C l$ from the coding region was amplified and was cloned into pDONR/Zeo plasmid (Invitrogen $\mathrm{GmbH}$, Karlsruhe, Germany) with BP clonase (Invitrogen $\mathrm{GmbH}$, Karlsruhe, Germany) according to manufacturer's instructions.

This vector generates sense and antisense fragment for silencing (Fig. 5). Using the LR clonase reaction (Invitrogen $\mathrm{GmbH}$, Karlsruhe, Germany) the silencing cassette is transferred to the destination vector pGS1 (Tran, 2011), which contains the hygromycin B phosphotransferase gene (hph) as selection marker.

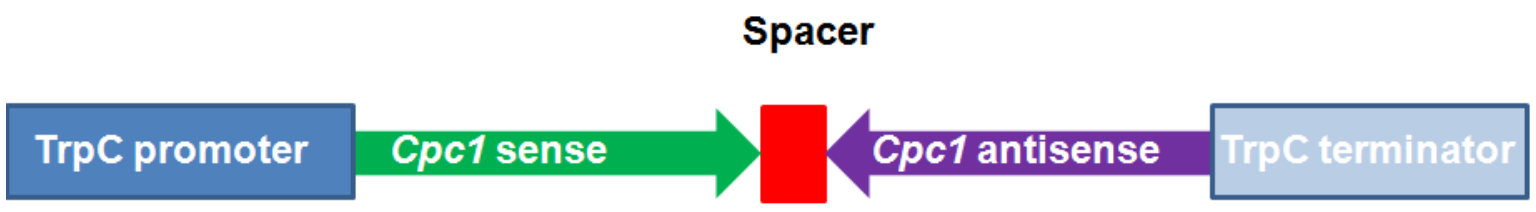

Fig. 5 silencing construct for RNAi of $\boldsymbol{V}$. longisporum. The hairpin construct consists of promotor, sense and antisense part, as well as terminator

This way the vector pGSCPCl was constructed and used as gateway silencing vector (Fig. $6)$. 

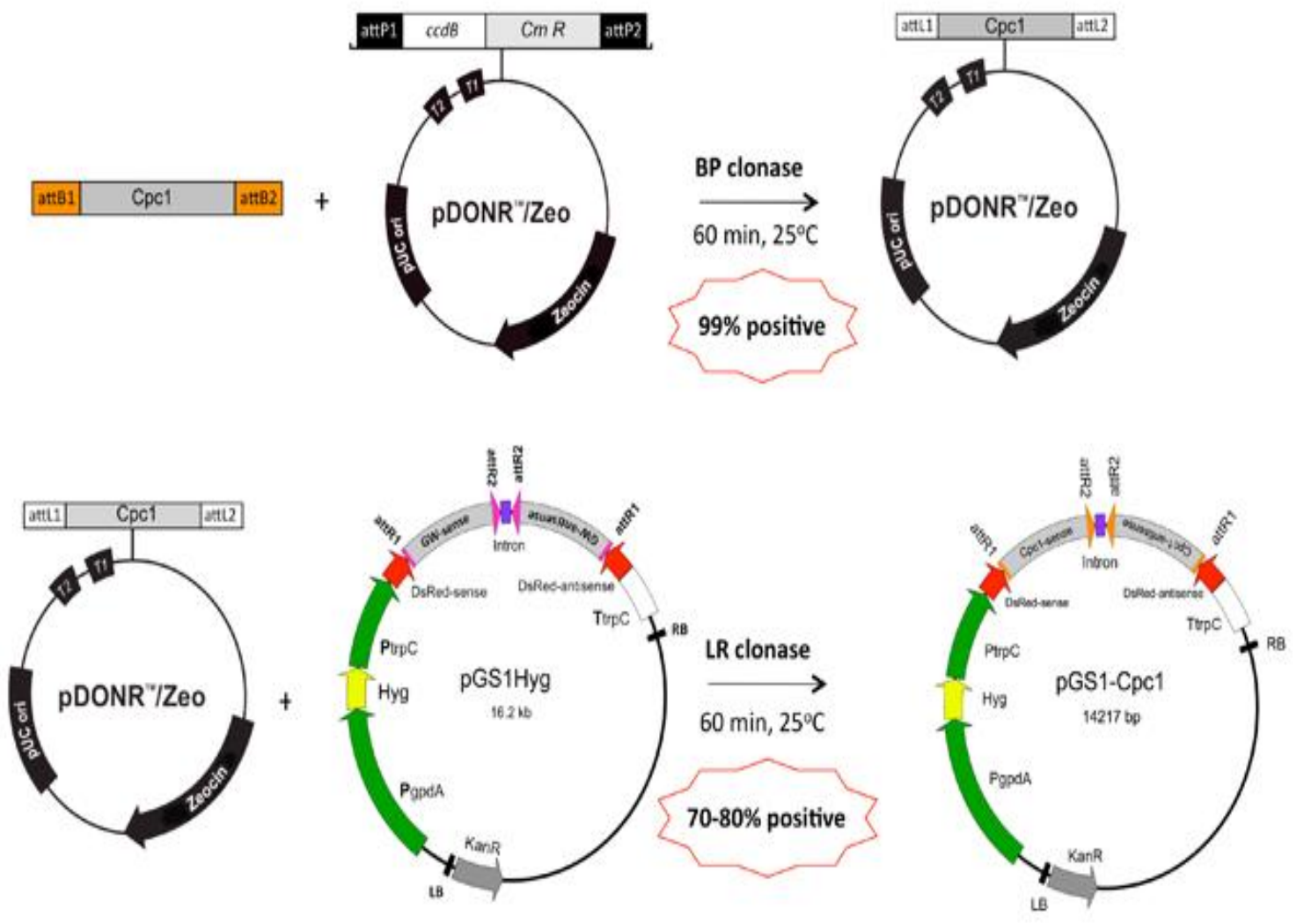

Fig. 6 Two-step procedure to create the hairpin RNA-mediated silencing construct forCpc1 gene. A fragment of $550 \mathrm{bp}$ was amplified from the $C P C 1$ transcripts. The PCR product with attB recombination sequences was transferred to the donor vector ( $\mathrm{pDONR} / \mathrm{Zeo}$ ) by $\mathrm{BP}$ clonase via a reaction between att $\mathrm{B}$ sequences and $a t t \mathrm{P}$ sequences of this vector. The reaction results in pDONR-Cpc1 vector (entry vector) with $a t t \mathrm{~L}$ recombination sequences. In presence of LR clonase, the entry vector exchanges the $C P C 1$ fragment with the gateway cassettes in pGS1Hyg via a reaction between att $\mathrm{L}$ sequences and att $\mathrm{R}$ sequences. This reaction creates the silencing construct for $C P C 1$ gene (pGS1-Cpc1i).

The generated plasmid (Fig. 7) was transformed into V. longisporum strain 43 by A. tumefaciens-mediated transformation (ATMT (Mullins et al., 2001)). A. tumefaciens AGL-1 strain (Lazo et al., 1990) was transformed with plasmids using LB medium containing kanamycin (100 $\mu \mathrm{g} \mathrm{ml-1).} \mathrm{Filter} \mathrm{paper,} 90 \mathrm{~mm}$ (Sartorius), was used for cocultivation of the A. tumefaciens culture and the $V$. longisporum spore suspension. The transformants were grown on medium lacking hygromycin B for five generations and then plated on selective medium supplemented with $100 \mu \mathrm{g} \mathrm{ml}-1$ hygromycin $\mathrm{B}$, where only mitotically stable transformants could grow (Singh et al., 2010). 


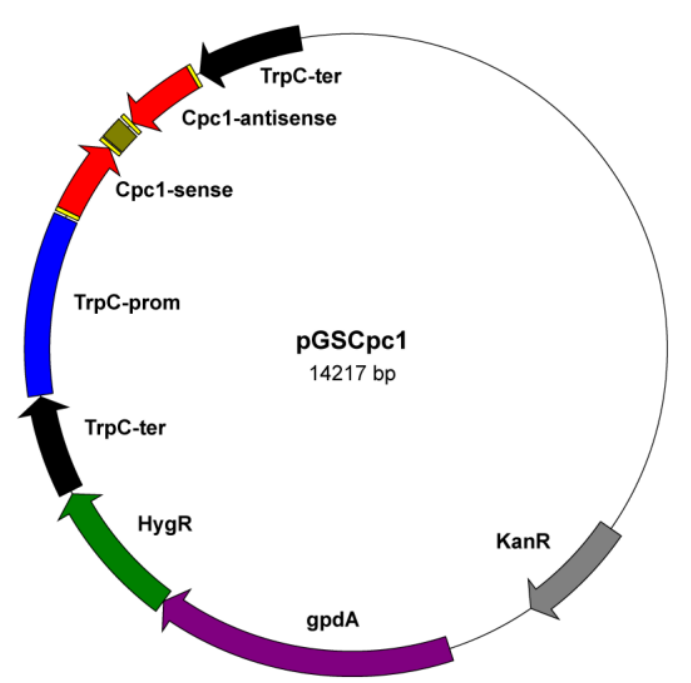

Fig. 7 Silencing construct for VICPC1. Vector is integrated with the gateway cassette in sense and antisense direction for generating hairpin RNA silencing constructs. The same gateway cassettes in both directions contains two special recombination sequences ( $a t t \mathrm{R} 1$ and $a t t \mathrm{R} 2)$. They will be replaced automatically with a target gene fragment via two reactions using BP clonase and LR clonase.

A vector for silencing of $L A E 1$ and $T A G L$ was generated in the same way as discribed for CPC1.

\subsection{Knockout strategy for $V$. dahliae}

For fungal species which genomes are not yet sequenced, silencing strategy remains probably the best option to study genes of interest in these organisms. However, silencing suppresses maximally about 80-90\% of gene expression in fungi (Nakayashiki, 2005; Janus et al., 2007; Singh et al., 2010). The rest activity of the gene (10-20\%) might be still enough for its function in the fungus. Though to analyze the complete functions of genes in Verticillium species, the gene must be either disrupted or removed from the genome. Because up to now, the Agrobacterium tumefaciens-mediated transformation represents the method with the highest efficiency for gene transfer into Verticillium species, strategies of gene disruption in Verticillium require a binary vector as a deletion cassette carrier. Gene disruption in $V$. dahliae was performed successfully by inserting a resistant cassette into open reading frames of genes using transposons (Dobinson et al., 2004; Rauyaree et al., 2005; Klimes and Dobinson, 2006) or by multi-step cloning using different plasmids (Tzima et al., 2010). The disadvantage of both strategies is that they are complicated and time-consuming. Therefore, we used a binary vector for gene disruption pKO2 (Tran; 2011) that can be used directly for making deletion constructs as well as for Verticillium 
transformation via Agrobacterium. This binary vector possess nourseothricin resistance gene as selection markers (Fig. 8), so it can be used to perform a single knockout in haploid species $V$. dahliae and $V$. albo-atrum. In V. longisporum, silencing of CPC1 resulted in a phenotype, which was not affected in growth rate, but was sensitive to amino acid starvation conditions when compared with the wild type phenotype. Moreover, pathogenicity inside the host plant was suppressed compared to wild type infection. So in parallel to silencing of $C P C l$ in $V$. longisporum we also decided to generate deletion mutants of this gene in $V$. dahliae. Therefore we used this new knockout vector to construct the cassettes for disruption of $C P C 1$ gene in $V$. dahliae.

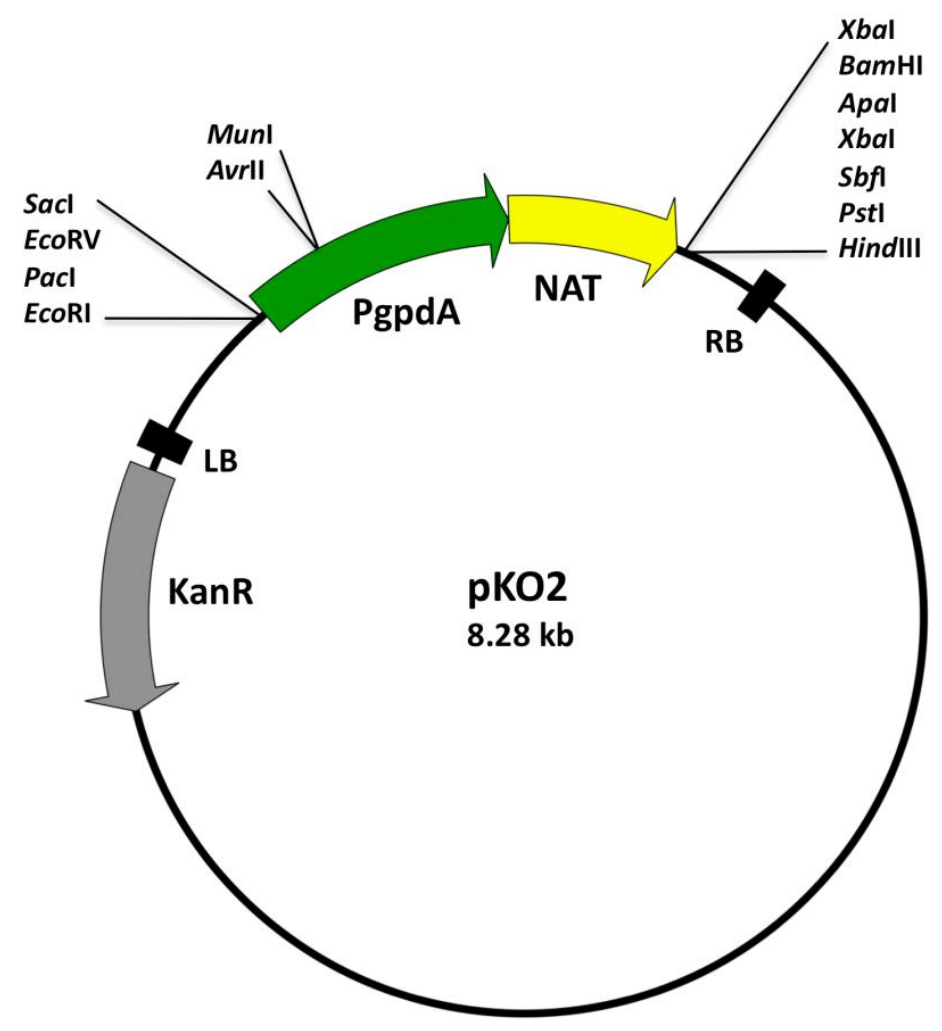

Fig. 8 The structure of plasmid pKO2 for gene disruption (Tran; 2011). pKO2 carries the nourseothricin resistance cassette. The vector possesses multiple cloning sites before and after the resistance cassettes that allow easy generation of constructs to disrupt genes of interest in Verticillium.

To generate knockout construct for $V$. dahliae the vector $\mathrm{pKO} 2$ was used to generate a CPC1 mutant allele for gene disruption. The vector was used to disrupt $C P C 1$ gene in $V$. dahliae. Two fragments of $1.6 \mathrm{~kb}$ and $866 \mathrm{bp}$ covering the $C P C 1$ gene from $V$. dahliae strain VdJR2 with a gap of 252 bp was amplified (Fig. 9) using the pairs VdcpcKOF11 and $V d c p c \mathrm{KOF} 1 \mathrm{r}$ and $V d c p c \mathrm{KOF} 21$ and $V d c p c \mathrm{KOF} 2 \mathrm{r}$. (Table 3) These primers contained 


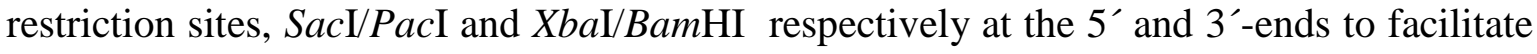
cloning of the PCR products. These products were aligned together with the resistance cassette to generate the cpc1 deletion construct. The resulting 3.7-kb CPC1 fragment was cloned into pKO2 at $E c o \mathrm{RV}$ and $\mathrm{Xba \textrm {I }}$ to create pKO2CPC1 vector (Fig. 9). This $V d C P C 1$ deletion construct was introduced into A. tumefaciens AGL1 for fungal transformation. The resulting transformants were screened by PCR and verified by Southern hybridization.

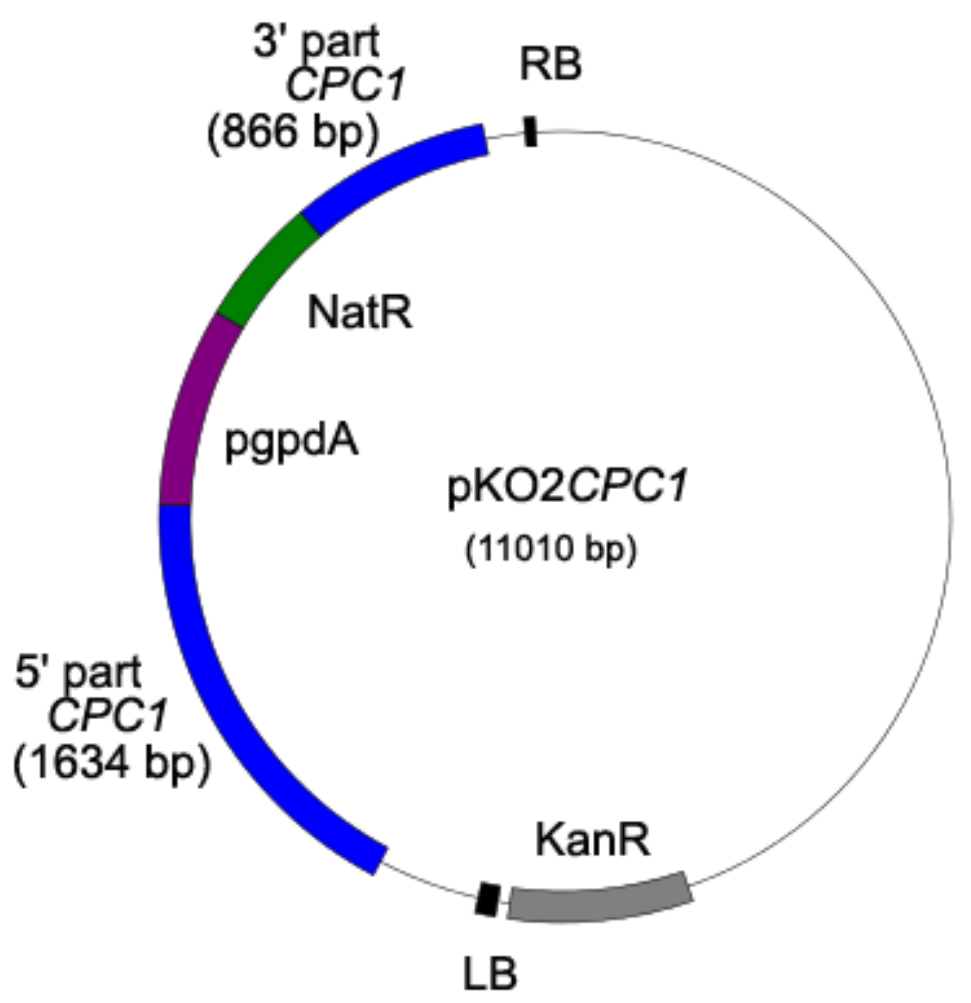

Fig. 9. Knockout construct for $\mathbf{V d C P C 1}$. The gene for $C P C 1$ is disrupted by Nat resistance cassette. The binary vector transfers the deletion cassette via A. tumefaciens mediated transformation into $V$. dahliae.

\subsection{Southern hybridization}

The Southern hybridization was performed following the recommendations in the provided manuals from GE Healthcare manufacturer. In brief, about $25 \mu \mathrm{g}$ of genomic DNA was digested for overnight with $3 \mu 1$ of an appropriate restriction enzyme that uncuts or cuts the target genes at a unique site outside the probe sequence. The digested mixture was analyzed on a $1 \%$ agarose gel, DNA was denaturated and transferred to the Amersham Hybond-N membrane (GE Healthcare, Munich, Germany) by blotting. DNA molecules 
and the membrane were cross-linked under UV light. A fragment (about $600 \mathrm{bp}$ ) of the target gene or the whole gene was amplified and labelled as probe using Amersham AlkPhos Direct labelling Reagent (GE Healthcare, Munich, Germany). The DNA on the membrane was hybridized to the specific probe in hybridization buffer at $60^{\circ} \mathrm{C}$ for overnight. The membrane was treated carefully with washing buffers to eliminate background. Then, chemiluminescent signals were detected using the Amersham CDP-Star Detection reagent (GE Healthcare, Munich, Germany).

\subsection{Quantitative real-time PCR}

The total RNA was extracted from $0.1 \mathrm{~g}$ of fungal mycelium grown in SXM medium using the RNeasy Plant Mini Kit (Qiagen, Hilden, Germany). Concentration of RNA samples was measured using NanoDrop-1000 spectrophotometer (PEQLAB, Erlangen, Germany). Then, $1 \mu \mathrm{g}$ of total RNA was transcribed into cDNA in $20 \mu \mathrm{l}$ using the QuantiTect ${ }^{\circledR}$ Reverse Transcription kit (Qiagen, Hilden, Germany). The expression level of a gene was measured by real-time PCR using a specific primer pair. Reactions contained $2 \mu \mathrm{l}$ of each $0.5 \mathrm{pM}$ primer (forward and reverse), $1 \mu \mathrm{l}$ of the cDNA, and reverse transcriptase-grade PCR water to a final volume of $12.5 \mu \mathrm{l}$. The 5 PRIME MasterMix (5 PRIME GmbH, Hamburg, Germany) was added to obtain a final running volume of $20 \mu \mathrm{l}$ per reaction. Each reaction was run in triplicate for both the standard and unknown samples. Reactions were run under the following conditions using the Light Cycler 2.0 System (Roche, Manheim, Germany): $95^{\circ} \mathrm{C}$ denaturation for $3 \mathrm{~min}, 42$ cycles at $95^{\circ} \mathrm{C}$ for $10 \mathrm{~s}, 63^{\circ} \mathrm{C}$ for $15 \mathrm{~s}$ and $72^{\circ} \mathrm{C}$ for $25 \mathrm{~s}$ to calculate cycle threshold values, followed by $95^{\circ} \mathrm{C}$ for $1 \mathrm{~min}, 55^{\circ} \mathrm{C}$ for $1 \mathrm{~min}$ and 80 times of $55^{\circ} \mathrm{C}$ for $10 \mathrm{~s}$, increasing temperature by $0.5^{\circ} \mathrm{C}$ each cycle to obtain melting curves and to enable data analyses. Standard curves were produced with purified DNA products of 10 and $1 \mathrm{pg} / \mu \mathrm{l}$ and starting concentrations of 100,10 , and $1 \mathrm{fg} / \mu \mathrm{l}$. A baseline subtracted curve fit was used to generate standard curve data. Absolute amounts of transcripts were calculated using a correlation coefficient formula generated from the standard curve in each run.

\subsection{Plant experiments}

\subsubsection{Plant infection assays}

Rapeseed Brassica napus, Arabidopsis thaliana or tomato seedlings of ten days old were inoculated with $10^{7}$ fungal spores/ml (mutants or wild type), and mock with tap water for 40 min by root-dipping. The seedlings were then transferred in pots with a sterile sand:soil (1:2) mixture. The plants were allowed to grow in a climate chamber with 16 hours light at 
$23^{\circ} \mathrm{C}$ and 8 hours dark at $20^{\circ} \mathrm{C}$. The height of plants or leaf size was measured to calculate disease scores at 7, 14, 21, 28 and 35 days post inoculation (dpi).

Spore injection: $50 \mu \mathrm{l}$ of $10^{7}$ fungal spore suspension (wild type or mutants) was transferred into each tomato plant of 3 weeks old by injection. For mock, the sterile tap water was used instead. The disease symptoms were observed at 14 days after injection.

\subsubsection{Quantification of fungal DNA from infected plants.}

Hypocotyls, stem (5-6 cm from top of plant), roots and leaves were separated from the infected rapeseed plants harvested at 28, and 35 dpi. 20 plants were harvested from each treatment and fungal DNA

For extraction of fungal DNA from infected plant the DNeasy Plant Mini Kit (Qiagen Hilden, Germany) was used with modification to manufacturers protocol. Plant material was grinded using a Retsch mill (Retsch, , Germany) at $30000 \mathrm{rpm}$ for $1 \mathrm{~min} 30 \mathrm{sec}$. 50-100 mg of grinded material was filled in an E-cup and solved in $400 \mu \mathrm{l} \mathrm{Buffer} \mathrm{AP1} \mathrm{and}$ $4 \mu \mathrm{RNase}$ A stock solution $(100 \mathrm{mg} / \mathrm{ml})$. The mixture was incubated for $10 \mathrm{~min}$ at $65^{\circ} \mathrm{C}$. and mixed 2 or 3 times during incubation by inverting tube. To lyse the cells $130 \mu \mathrm{l}$ Buffer AP2 was pipetted to the lysate, and incubated for 5 min on ice. The samples were centrifuged for $5 \mathrm{~min}$ at $10000 \mathrm{rpm}$.

After centrifugation the supernatant was transfered to a QIAshredder Mini spin column and centrifuged for $2 \mathrm{~min}$ at $10000 \mathrm{rpm}$. The flow-through fraction was pipetted into a new tube without disturbing the pellet. 1.5 volumes of Buffer AP3/E was added to the cleared lysate, and mixed by pipetting. The mixture was transferred into the DNeasy Mini spin column and centrifuged for $1 \mathrm{~min}$ at $8000 \mathrm{rpm}$. The column was placed into a new $2 \mathrm{ml}$ collection tube, and $500 \mu \mathrm{l}$ Buffer AW was added. The samples were centrifuged for $1 \mathrm{~min}$ at $8000 \mathrm{rpm}$. The flow-through was discarded, and an additional $500 \mu \mathrm{l}$ Buffer AW was added to the DNeasy Mini spin column. The columns were centrifuged for $2 \mathrm{~min}$ at 10000 rpm to dry the membrane. The DNeasy Mini spin column was transferred to a $1.5 \mathrm{ml}$ E-cup and $50 \mu \mathrm{l}$ of $\mathrm{H}_{2} \mathrm{O}$ was pipetted on the column and incubated for $5 \mathrm{~min}$ at room temperature. For eluation the samples were centrifuged at $8000 \mathrm{rpm}$. To increase the amount of eluated DNA an additional $50 \mu \mathrm{l}$ of $\mathrm{H} 2 \mathrm{O}$ was added to the column and centrifugation was repeated. The concentration of the samples was measured using NanoDrop ND-1000 spectrophotometer (PEQLAB, Erlangen, Germany). The samples were stored at $-20^{\circ} \mathrm{C}$. 
The expression level of a gene was measured by real-time PCR using a specific primer pair. Reactions contained $2 \mu \mathrm{l}$ of each $0.5 \mathrm{pM}$ primer (forward and reverse), $1 \mu \mathrm{l}$ of the DNA sample, and reverse transcriptase-grade PCR water to a final volume of $11 \mu \mathrm{l}$. The 5 PRIME MasterMix (5 PRIME GmbH, Hamburg, Germany) was added to obtain a final running volume of $20 \mu \mathrm{l}$ per reaction. Each reaction was run in triplicate for both the standard and unknown samples. Reactions were run under the following conditions using the Light Cycler 2.0 System (Roche, Manheim, Germany): $95^{\circ} \mathrm{C}$ denaturation for $3 \mathrm{~min}, 42$ cycles at $95^{\circ} \mathrm{C}$ for $10 \mathrm{~s}, 63^{\circ} \mathrm{C}$ for $15 \mathrm{~s}$ and $72^{\circ} \mathrm{C}$ for $25 \mathrm{~s}$ to calculate cycle threshold values, followed by $95^{\circ} \mathrm{C}$ for $1 \mathrm{~min}, 55^{\circ} \mathrm{C}$ for $1 \mathrm{~min}$ and 80 times of $55^{\circ} \mathrm{C}$ for $10 \mathrm{~s}$, increasing temperature by $0.5^{\circ} \mathrm{C}$ each cycle to obtain melting curves and to enable data analyses. The amount of DNA of $V$. longisporum was estimated by integration of a calibration curve using increasing amounts of genomic V. longisporum DNA from $100 \mathrm{pg}$ to $100 \mathrm{ng}$, in the analysis.

\subsection{DNA-sequencing}

DNA-sequences were analysed using the Sanger-method (Sanger and Coulson, 1975) in Goettingen Genomics Laboratory. The concentration of the DNA was measured using Nano drop (NanoDrop Spectrophotometer ND-1000, PEQLAB BIOTECHNOLOGIE GMBH, Erlangen,D). Then, the necessary amount of DNA was calculated to get a final concentration of $50 \mathrm{ng} / \mu \mathrm{l}$ to $100 \mathrm{ng} / \mu \mathrm{l} .200 \mathrm{ng}$ of DNA together with $5 \mathrm{pmol}$ of primer in a total volume of $5 \mu \mathrm{l}$ was given for sequencing. 


\section{Chapter 3. Results}

\subsection{Cross pathway control and its role in $V$. longisporum}

Microorganisms have to adjust to altering environmental circumstances. To survive under rapidly evolving environmental situations, they need to respond on these impacts. The Cross pathway control $(C p c)$ of amino acid biosynthesis acts in response to the environmental stress conditions amino acid deprivation. $C p c$ has been shown to play a crucial role for fungal life cycle and metabolism. In other organism $\mathrm{Cpc}$ is involved in many cellular processes regarding primary and secondary metabolism. The Verticillium cross pathway control was upregulated when the fungus is growing inside the plant (Singh et al. 2010). However the investigation whether CPCl is involved in pathogenicity of the pathogen is still not known. Therefore we had a deeper look into the role of $C P C l$ in $V$. longisporum and $V$. dahliae, and to see if mutants lacking CPCl by silencing of the gene or by deletion are affected in their pathogenicity. We hypothesized that an active amino acid biosynthetic capability would be required for survival in the host and, therefore, that the $V$. longisporum cross pathway control would play an important role in the pathogenicity of this fungal pathogen.

\subsubsection{V. longisporum has two copies of VICPC1}

$V$. longisporum has been described as hybrid between two Verticillium species (Inderbitzin et al. 2011; Tran et al. 2013). Therefore it was examined whether VlCPC1 has two isogenes. To determine the copy number of VlCPCl Southern analysis was performed (Fig. 10). So genomic DNA of V. longisporum, V. dahliae, and V. albo-atrum were digested with suitable enzymes, and subjected to Southern hybridization. A fully sequenced $550 \mathrm{bp}$ long fragment of VlCPCl, amplified with primers ProbeCpc1left and ProbeCpc1right was used as the probe. In our several independent Southern hybridization analyses, two signals were generated for $V$. longisporum as compared to $V$. dahliae, and $V$. albo-atrum for the genomic DNA digested with BamHI. According to these data two isogenes for cross pathway control (VlCPC1-1 and VlCPC1-2) exist. 


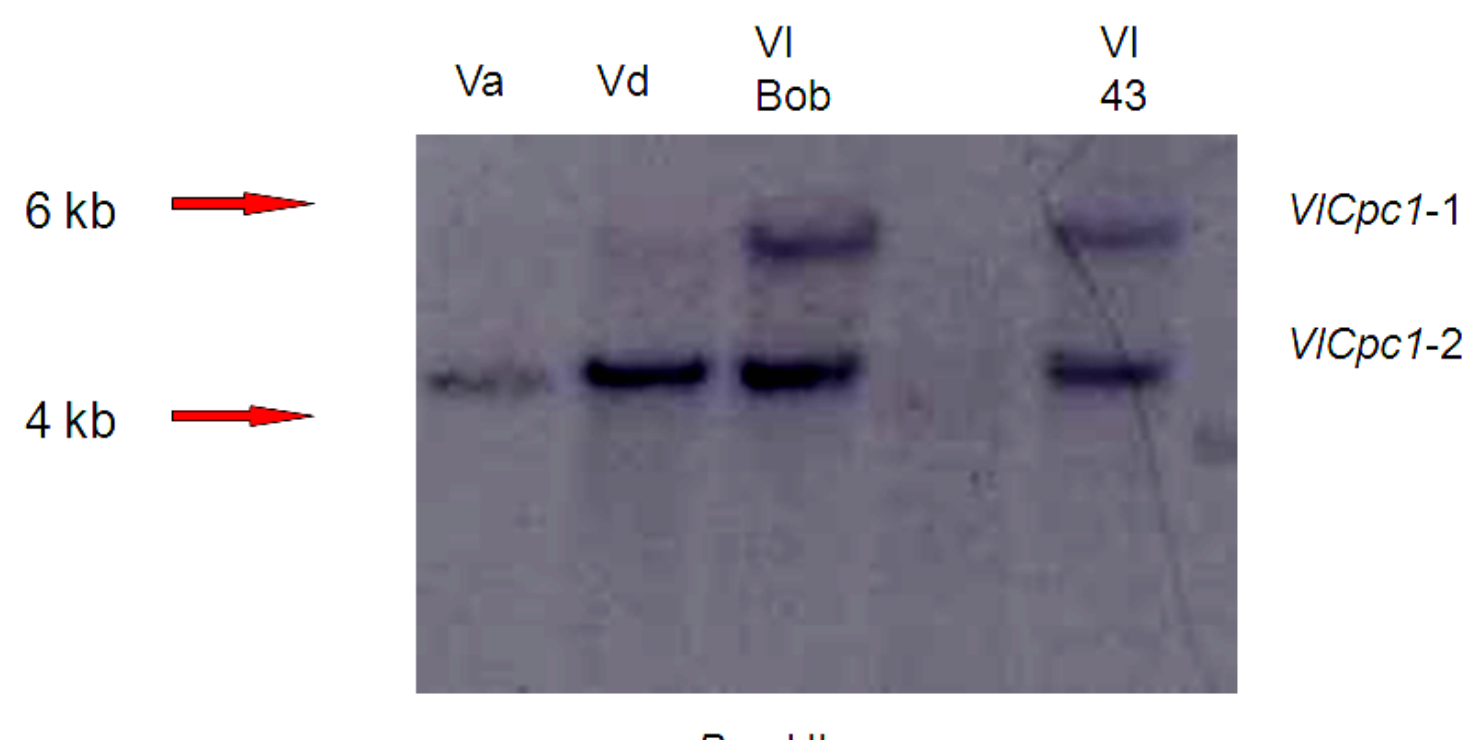

$\mathrm{BamHI}$

Fig. 10 Determination of the isogene VICPC1-2 of VICPC1-1. Southern hybridization analysis of V. alboatrum, V. dahliae and V. longisporum. The genomic DNA was digested with BamHI. A 500 bp sequence of $V l C P C 1-1$ was used as a probe. For $V$. albo-atrum and $V$. dahliae one copy of CPCl is visible, for $V$. longisporum two copies exist.

These results demonstrated that $V l C P C l-1$ has an isogene, VlCPCl-2. To determine the extent of similarity of these two isogenes in the $V$. longisporum genome, the genomic DNA of V. longisporum was digested with SalI and XhoI and run on a low percentage agarose gel. DNA was extracted from the four gel fragments corresponding to spots where the signals were generated in the Southern hybridization analysis (Fig. 10). VlCPC1-1 specific primers were used to amplify the gel extracted DNA and subjected to sequencing. Sequencing of a $V$. longisporum cDNA library (Singh et al., 2010) revealed the presence of both transcripts (VlCPC1-1 and VlCPC1-2) for the cross pathway transcriptional regulator VlCPC1. We examined the chromosomal loci of the corresponding CPC1 genes of the haploids V. dahliae and V. albo-atrum in comparison to V. longisporum (Fig. 10), which has been demonstrated to be a hybrid between two different Verticillium species (Inderbitzin et al., 2011). In a first approach, we designed a primer pair CPC1Seqleft and CPC1Seqright (Table 3) on the basis of the CPC1 locus (VDAG_10113) from the BROAD $V$. dahliae genome (Klosterman et al., 2011). Sequencing of the PCR products from $V$. dahliae and $V$. albo-atrum resulted in the unique single sequences of $725 \mathrm{bp}$ for $V d C P C 1$ and 723 bp for $V a C P C 1$. In contrast, cloning and sequencing of the PCR product from $V$. longisporum revealed two distinct sequences for $V l C P C l$ corresponding to isogenes VlCPC1-1 and VlCPC1-2 with the lengths of 725 and 723 bp similar to VdCPC1 and $V a C P C 1$, respectively (Fig. 11 and 12). The DNA sequences were determined and resulted in two, VlCPC1 and a single CPC1 sequences for $V$. dahliae or V. albo-atrum, 
respectively (Fig. 11). In total there are more than 60 SNPS between all four sequences. The deduced open reading frames of all genes consist of two exons with a length of 13 or 653 base pairs (bp) separated by one intron. The introns of VdCPCl of V. dahliae and VICPC1-1 of V. longisporum have a length of $59 \mathrm{bp}$, whereas V. albo-atrum VaCPC1 and the second isogene $V l C P C l-2$ share a length of $57 \mathrm{bp}$. On sequencing of both loci it was found out that the coding and intronic sequences of both isogenes are identical in $V$. longisporum.

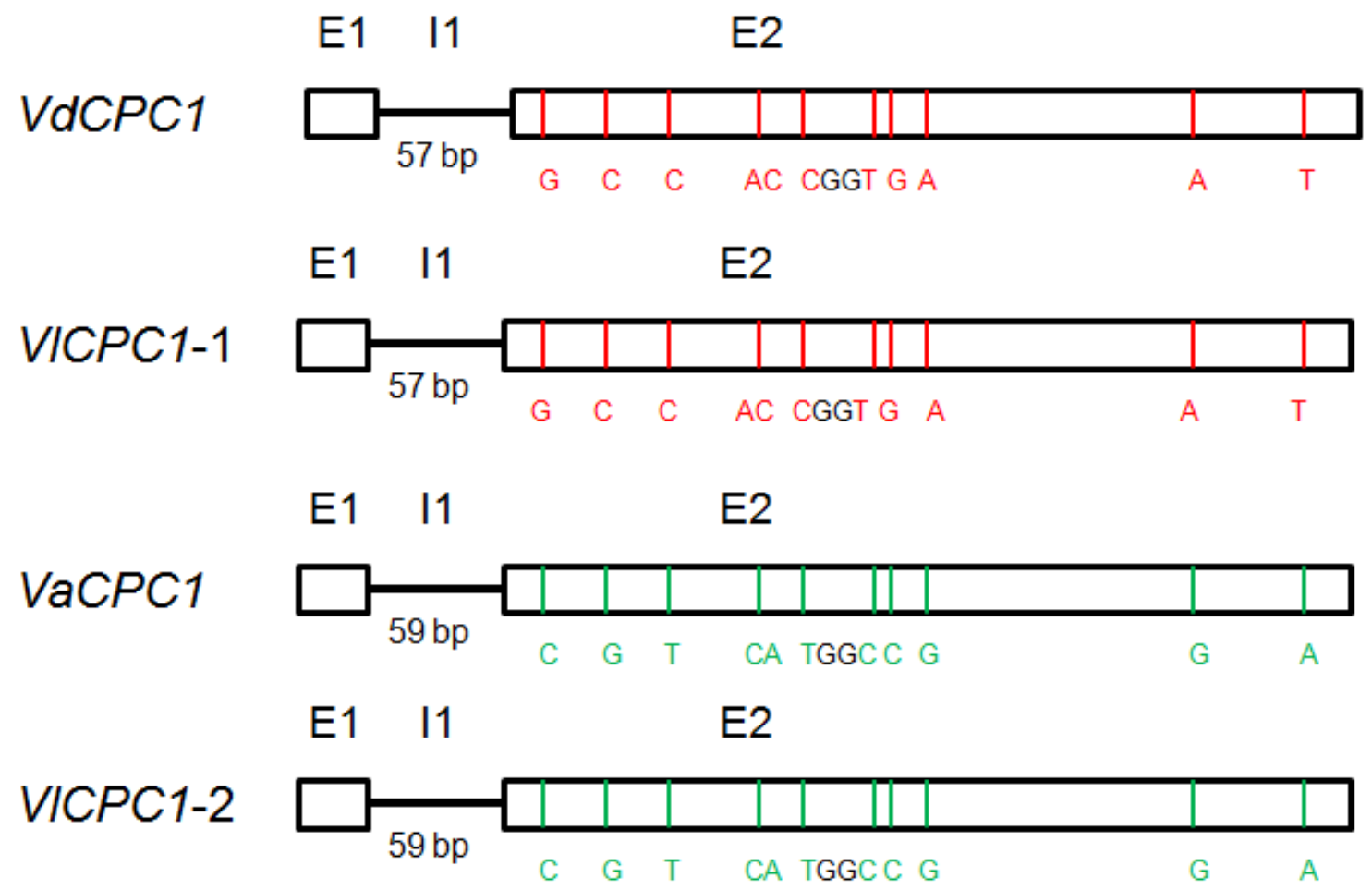

Fig. 11 Schematic of $C P C 1$ genes of Verticillium species. Gene comparison of $V$. longisporum sequences VlCPC1-1 and VlCPC1-2, V. dahliae VdCPC1 and V. albo-atrum VaCPC1. Colors indicate common nucleotides between VlCPCl-1 and VdCPCl (red) or VlCPCl-2 and VaCPCl (green). 


\section{A}

$\operatorname{VACPC1}$

VICPC1-1

$\operatorname{VaCPC1}$

ATGAACATCGCGGGTGAGTGCCCAAGAAAGGGATTGATCTAGTTATACATCAGCTCACAT 60

ATGGACATCGCGGGTGAGTACCCAAGAAATGGAGTGACCCAGTCACACATCAGCTCACA- 59 ATGAATATCGCGGGTGAGTGCCTGAAATGTGGATTGATCCAGTCATACATCAGCTCACA-

GATTGCCTGTAGATTTCGAAGAATTCACCGGGTTCGAGGGTGGGGCATCGACTGCCTACT 120 VICPC1-1 AATTGCCTGTAGATTTCGAAGAATTCACCGGGTTCGAGGGTGGGGCATCGACTGCCTACT 120 $\begin{array}{lll}\text { VICPC1-2 } & \text {-AATGCCTGTAGATTTCGAAGAATTCACCGGGTTCGAGGGTGGGGCATCCACTGCCTACT } 118 \\ \text { VaCPC1 } & \text {-AAGGCCTATAGACTTCGGGAATTACAGGGTCGAGGGTGGGCATCCACTGCCTACT } 118\end{array}$

CGTCGCCCAGCGCGCCTTCCGTGTTCGATATGCCGAGTGGATCCCACAACATCGGTACTG 180 VICPC1-1 CGTCGCCCAGCGCGCCTTCCGTGTTCGATATGCCGAGTGGATCCCACAACATCGGTACTG 18 VICPC1-1 CGTCGCCCAGCGCCCCTTCGGTATTCGATATGCCAAGTGGATCCCACAACATTGGTACTG 178

VdCPC1 TTTCCCCACAGGACCTCTTAATCCAGGATCCATACATGTCTGCGCCGAACTCAACGGCAT 240 VICPC1-1 1 TICCCACAGGACCTCTTAATCCAGGATCCATACATGTCTGCGCCGAACTCAACGGCAT 240 VICPC1-2 TTTCCCCACAGGACCTCTTAATCCAGGATCCATACATGTCTGCGCCGAATTCAACGGCAT 23 $\operatorname{VaCPC1}$

$\begin{array}{ll}\text { VdCPC1 } & \text { TCACAGCCTTGACGTCGCCTTCGCCGTTCGAATCGCCAGAATATATGGATGGCTATGACG } 300 \\ \text { V1CPC1-1 } & \text { TCACAGCCTTGACGTCGCCTTCGCCGTTCAATCGCCAGATTATATGGATGGCTATGACG } 300\end{array}$ VICPC1-1

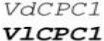

VICPC1-2

$\operatorname{VaCPC1}$ TCACCGCCTTGACATCGCCTTCGCCATTCGAATCGCCAGAATATATTGATGGCTACGAAG 298

TGTCGCCAAACTTTGGAAGCTCGGGAGAATATGATGTGGCCGGAGCCAGTGGTTCTCAC 360 TGTCGCCAAACTTTGGAAGCTCGGGAGAATATGATGTGGCCGGCAGCCAGTGGTTCTCAC 360 TGTCCCCAAACTTTGGAGGCTCGGGAGAATTTGATGTGGCCAGCAGCCAGTGGTTTTCAC 358 TGTCGCCAAACTTTGGGGGCTCTGGAGAATTCGATGTGGCTAGCAACCAGTGGTTCTCAC 358

VACPC1 TTTTCCCTGACCAGAACGCGAGTGTCGAGACAACGGGGGCGGAACAGCCGCTCGGGGACA 420 ICITCCIGACCAGAACGCGAGTGTCGAGACACAGGGGACGGAACAGCCCCTTGGGCACC 418 TTTTCCCTGACCAGAACGCGAGTGTCGAGACACAGGGGGCGGAGCAGCCGCTTGGGCACA 41

VdCPC1 GAGTCCAGAACAGCACGGTAAGCGAGTGCCCTCGCCGTAAATCGAGTGGCTCGCCTGCTT 480 作

TCGTTCAAACCA ATGTTCAGAACAGCTTGGCAAGCGGGTCCCCCCGCGTAAGTCGAGTGCCTCGCCTGCTA 478

CTGGGCGGCACTCGTCAGTGGCTGGCGTCAACGCGAGGAAGCGAGACAAGCCCCTGCCAC 540 $V A C P C 1, C T G C$

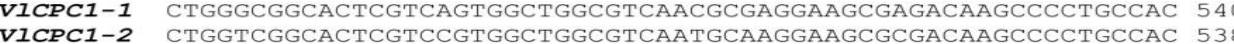

CTGGGCGGCACTCGTCGGTGGCTGGCGTCAACGCGAGGAAACGAGACAAGCCTCTGCCAC 53
VdCPC1 CCATCGTTGTTGAGAATCCAGATGACGTTGTAGCGATGAAACGCGCTCGCAACACTCTGG 600 VICPC1-1 CCATCGTTGTTGAGAATCCAGATGACGTTGTAGCGATGAAACGCGCTCGCAACACTCTGG 600 $\begin{array}{lll}V I C P C 1-2 & \text { CCATCGTTGTTGACAATCCGGATGACGTTATAGCGATGAAACGTGCTCGCAACACTCTGG } 598 \\ \text { VaCPCI } & \text { CCATCGTTGTGATAATCCGATGACGTTGTGGCATGAAGCGCGCTCGCAACACTCTGG } 598\end{array}$

VdCPC1 CAGCCCGCAAGTCTAGGGAGCGTAAAATGCAGCGGTTCGAGGATCTGGAGGAGAAGATTC 660 VICPC1-1 CAGCCCGCAAGTCTAGGGAGCGTAAAATGCAGCGGTTCGAGGATCTGGAAGAGAAGATTC 660 VICPC1-2 CAGCCCGCAAATCTAGGGAGCGTAAAATGCAGCGGTICGAGGATCTGGAGGAGAAAATIC 658 VaCPC1 CAGCCCGTAAGTCTCGAGAGCGTAAAATGCAGCGGTTCGAGGATCTGGAGGAAAAGATTC 65

VdCPC1 GCAAGTTGGAGGCGGAACGTGATCACTGGAAGACCTTGGCTTTGGGTCAGTCAACCAGCG 720 VICPC1-1 GCAAGTTGGAGGCGGAACGTGATCACTGGAAGACCTTGGCTTTGGGTCAGTCAACCAGCG 720 VICPC1-2 GCAAGTIGGAGGCGGAACGIGATCACIGGAAGACCATGGCTTTAGGTCAGTCAACTAGCG 718 VACPC1 GCAAGTTGGAGGCGAACGTGATCACTGGAAGACGATGGCTTTGGGTCAGTCGACCACCG 718

VACPCI ACTAG 725

VICPC1-1 ACTAG 725

$\begin{array}{lll}\text { VICPC1-2 } & \text { ACTAG } 723 \\ \text { VaCPC1 } & \text { ACTAG } 723\end{array}$

\section{B}

$\checkmark$ dCPC1 MNIADFEEFTGFEGGASTAYSSPSAPSVFDMPSGSHNIGTVSPQDLIIQDPYMSAPNSTA 60 VICPC1-1 MNIADFEEFTGFEGGASTAYSSPSAPSVPMPSGSHNIGTVSPQDLLIQDPYMSAPNSTA 60 VTCPC1 1 MNIADFEEFTGFEGGASTAYSSPSAPSVEDMPSGSHNIGTVSPODLLIODPYMSAPNSTA 60

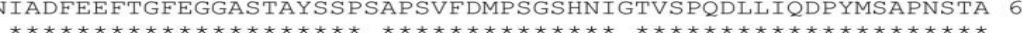

VdCPC1 FTALTSPSPFESPEYMDGYDVSPNFGSSGEYDVAGSQWFSLFPDQNASVETTGAEQPLGD 120 VICPC1-1 FTALTSPSPFESPEYMDGYDVSPNFGSSGEYDVAGSQWFSLFPDQNASVETTGAEQPLGD 120 VaCPC1 FTALTSPSPFESPEYIDGYEVSPNFGGSGEFDVASSQWFSLFPDQNASVETQGAEQQLEH 120

VACPC1 RVQNSTVSECPRRKSSGSPASGRHSSVAGVNARKRDKPLPPIVVENPDDVVAMKRARNTI 180 VICPC1-1 RVQNSTVSESPRRKSSGSPASGRHSSVAGVNARKRDKPLPPIVVENPDDVVAMKRARNTL 180

Vacpc1

$$
\begin{gathered}
* * * \\
\text { bzIP domain }
\end{gathered}
$$

ARKSRERKMQRFEDLEEKIRKLEAERDHWKTLALGQSTSD 22

ARKSRERKMQRFEDLEEKIRKLEAERDHWKTLALGQSTSD 221 998 $791 \%$

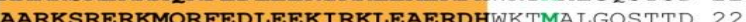

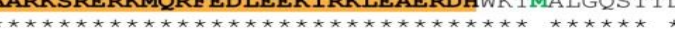

Fig. 12 Sequence alignment for $C P C 1$ genes of Verticillium species. Sequence alignments of $V$. longisporum sequences VlCPCl-1 and VlCPCl-2, V. dahliae VdCPCl and V. albo-atrum $\mathrm{VaCPCl}$. The grey box indicates intron regions and the bZIP region is orange. Additional colors indicate common nucleotides between $\mathrm{VlCPCl}-1$ and $\mathrm{VdCPCl}$ (red) or $\mathrm{VlCPCl}-2$ and $\mathrm{VaCPCl}$ (green). 
The comparison of the Verticillium derived genes revealed that V. albo-atrum and V. dahliae CPC1 are $88 \%$ identical and differ in 65 SNPs (Fig 11). The SNPs result in a change of 25 amino acids in the primary protein sequence and therefore the deduced amino acid sequence is $93 \%$ identical (Fig. 12). V. longisporum VlCPC1-1 differs in 12 and VlCPCl-2 in 36 nucleotides from the single $V$. dahliae $V d C P C 1$ with a remaining identical DNA sequence of $98 \%$ and $95 \%$, respectively (Fig. 12). This results in a change of 2 amino acids in the primary protein sequence of $\mathrm{VlCPCl}-1$ and therefore the deduced amino acid sequence is $99 \%$ identical for $V l C P C l-2$ there is a change of 12 amino acids in the primary protein sequence and therefore the deduced amino acid sequence is $95 \%$ identical. VlCPCl-1 has $88 \%$ identity with $V d C P C 1$, whereas VlCPC1-2 has at least $92 \%$ identity. There are two characteristic sequence patterns which correspond either to V. dahliae or to V. albo-atrum. VlCPC1-1 and $V$. dahliae $V d C P C l$ share the $\underline{\mathrm{CGGCAG}}$ signature which is different from the TAGCA $\underline{\mathrm{A}}$ signature which is present in VlCPCl-2 and V. albo-atrum VaCPCl (Figure 12). However this signature does not change the amino acid sequence. These data further corroborate that $V$. longisporum is a hybrid with two different copies for $C P C 1$, whereas haploids as $V$. dahliae or $V$. albo-atrum carry a single copy. The closest relatives are the $V$. dahliae copy and $V$. longisporum VlCPC1-1

Transcript analysis suggested that $C P C 1$ mRNA is induced during infection of B. napus and A. thaliana (Singh et al. 2010). We were interested in analyzing the importance of this plantspecific induction of $C P C 1$ for fungal pathogenicity on plants. Since we found two $C P C 1$ genes we analysed whether both of them carry signals which suggest a regulatory control of gene expression. Verticillium CPCl genes carry a cross-pathway recognition element in their own promoter (Fig. 13). The cross-pathway recognition element (CPRE) as binding site for target promoters of $C P C 1$ is conserved and had been originally described for Gcn $4 p$ as the CPCl orthologue of S. cerevisiae. The CPRE consists of a palindromic sequence element constituted by a central C.G pair that is flanked by TGA halfsites (Oliphant et al., 1989). In contrast to the yeast GCN4 gene which is not significantly autoregulated (Albrecht et al., 1998), the characterized Aspergillus and N. crassa CPCl homologs carry CPREs within their own promoter region for enhanced expression by autoregulation (Hoffmann et al., 2001; Krappmann et al., 2004; Tian et al., 2007). Inspection of the four chromosomal CPC1 genes of Verticillium revealed a typical CPRE motif TGACTCA which is present 985 bp upstream of the ORFs for the four CPCl genes of V. longisporum, V. dahliae and V. albo-atrum suggesting that they are also autoregulated by the transcription factor CPCl (Fig. 13A). 
The fungal $G C N 4 / C p c A / C P C 1$ genes are paradigms for translational control by small open reading frames (uORFs). In the region upstream of the translational start codon, a small ORF was identified, uORFa (-424 to $-304 \mathrm{bp}$ upstream), encoding putative polypeptide of 39 amino acids in length. The deduced primary sequence of an UORF shows high similarity to those mapped in the leader regions of CPCl from N. crassa (Paluh et al., 1988) with a calculated homology of 57\% (Fig. 13B).

The c-terminal leucine zipper region is highly conserved in filamentous fungi (Fig. 13C). The sequences of $V l C P C l-1$ and $V d C P C l$ are $100 \%$ identical, whereas compared to VlCPCl-2 and $\mathrm{VaCPCl}$ are $97 \%$ similar to this sequence. They differ in 2 amino acids. Compared to N.crassa the sequence is $74 \%$ identical and to A. nidulans, A.fumigatus and A. niger it is 47 $55 \%$ similar. 
A

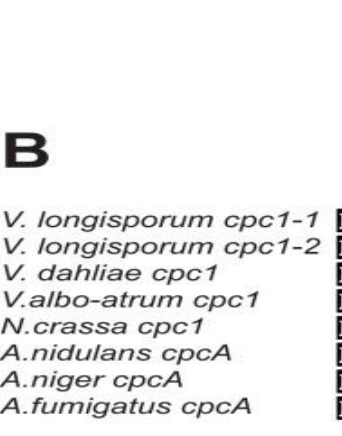

V. Iongisporum $c p c 1-1$
V. Iongisporum $c p c 1-2$
V. dahliae $c p c 1$
V.albo-atrum $c p c 1$
N.crassa $c p c 1$
A.nidulans $c p c A$
A.niger $c p c A$
A.fumigatus $c p c A$

T(CPRE)

C

V. longisporum cpc1-1 V. dahliae cpc1

V.albo-atrum cpc1

N.crassa $c p c 1$
A.nidulans $c p c A$

A. niger cpcA
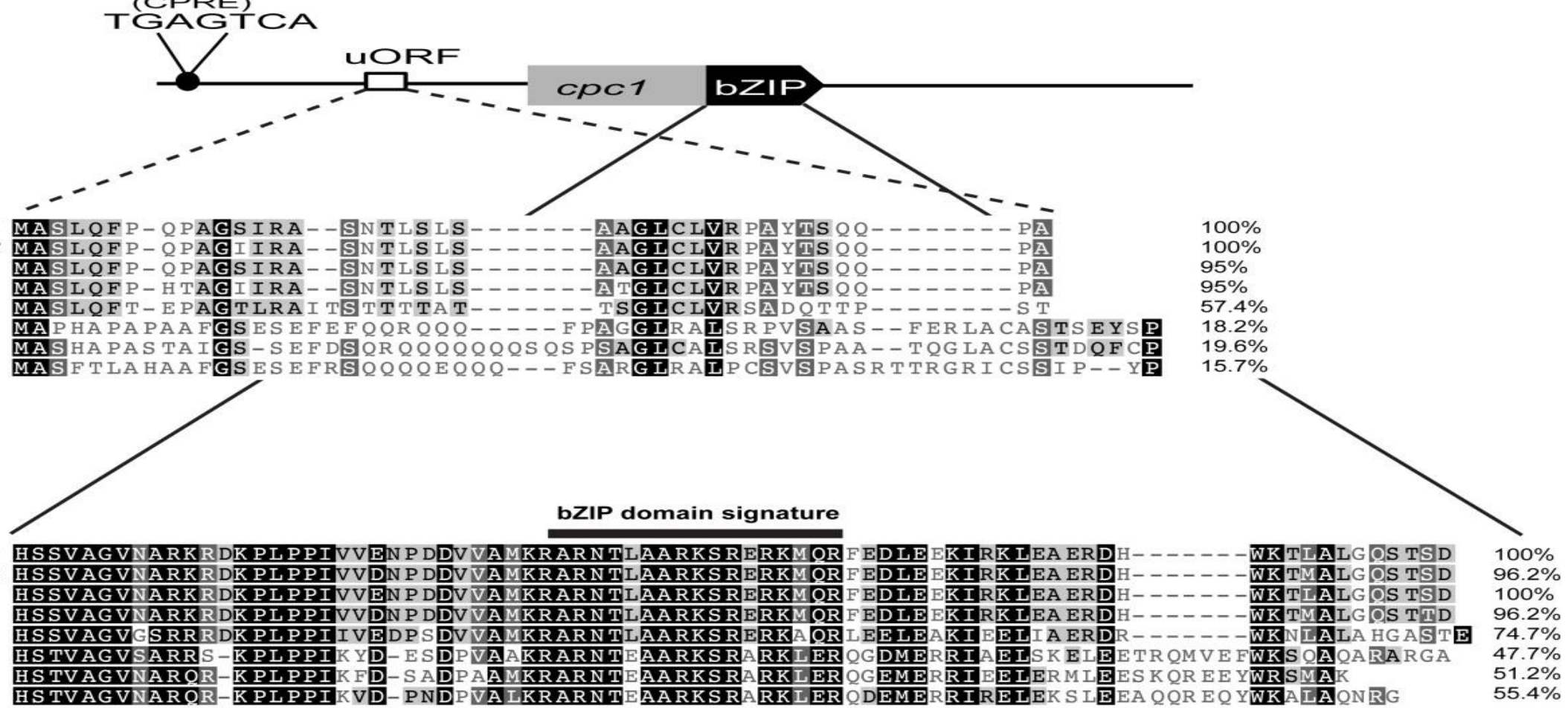

Fig. 13 The CPC1 loci of Verticillium species. A. The black dot indicates the putative cross-pathway control recognition element (CPRE) with the sequence 5'TGAGTCA3'. The conserved leucine zipper region at the C-terminus of $C P C l$ is in black. The upstream open reading frame uORF in the 5' leader region is represented as white box. B. uORF alignments of the deduced amino acid sequences in comparison to corresponding uORFs of CPCA/CPCl genes of A. fumigatus, A. nidulans, A. niger or $N$. crassa. White letters in black boxes represent conserved, grey boxes partially conserved amino acid residues. C. Alignments of the C-terminal leucine zipper regions of the same genes as in B. The bZIP transcription factors basic domain signature (InterProScan PS00036) is indicated as black line. (reprinted from Timpner et al. 2013 The Cpc1 regulator of the cross-pathway control of amino acid biosynthesis is required for pathogenicity of the vascular pathogen Verticillium longisporum. Mol Plant Microbe Interact doi.org/10.1094/MPMI-06-13-0181-R) 
An overall comparison of the four CPC1 genes from $V$. longisporum, $V$. dahliae,

$V$. albo-atrum with other species group them together with other pathogenic fungi like

Colletotrichium or Fusarium and separate them from saprophytes as Aspergillus or single cell yeasts S. cerevisiae (Fig. 14).

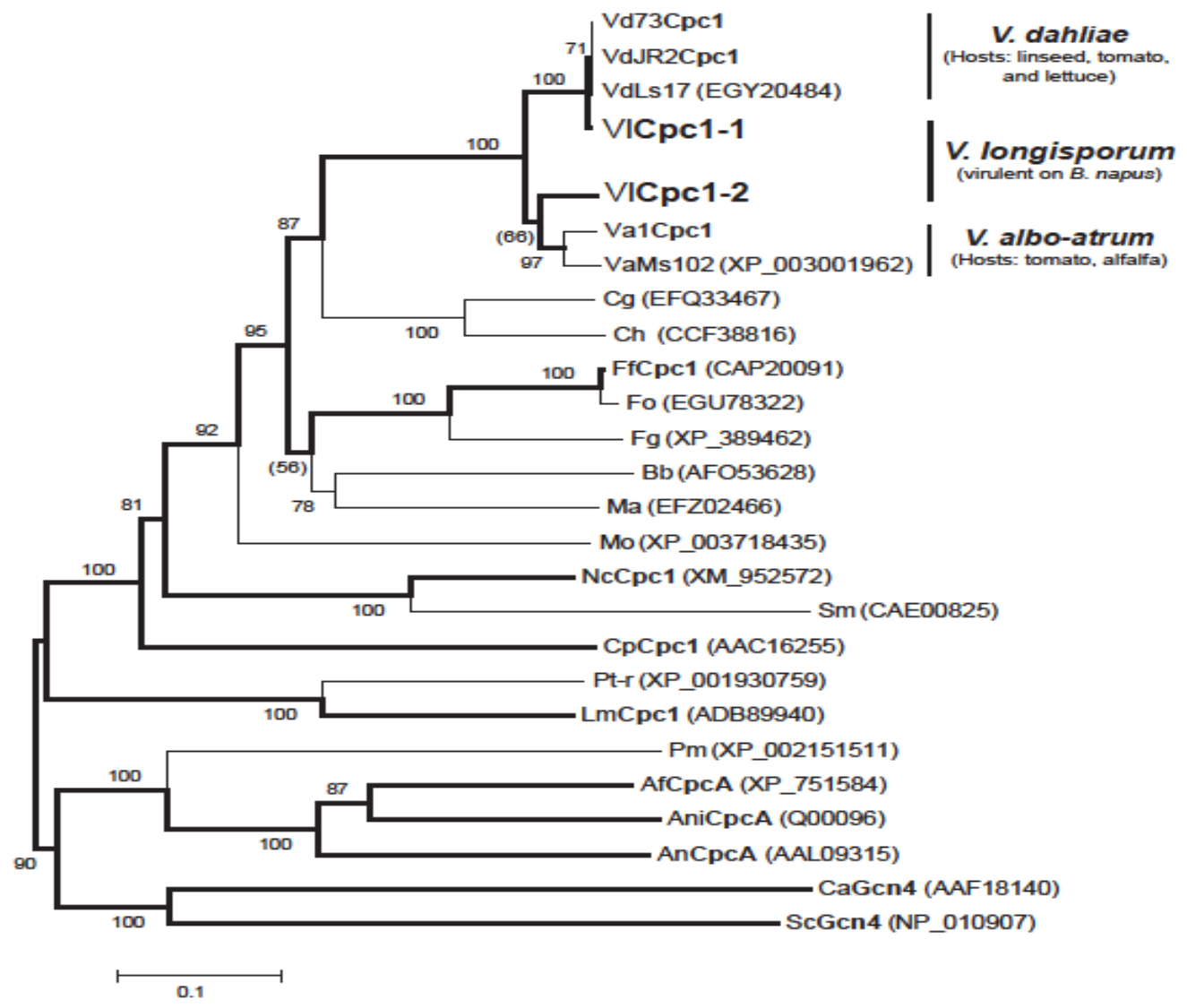

Fig. 14 Phylogenetic analysis for deduced Cpc1 proteins of three Verticillium species in comparison to the orthologues of other filamentous fungi. The virulent hybrid pathogen $V$. longisporum possesses two isogenes encoding deduced Cpc1-1 or Cpc1-2 which are similar to V. dahliae and V. albo-atrum, respectively. Other Cpc1-like proteins were deduced from the encoding genes with the provided accession numbers (in brackets), which were extracted from GenBank. Thick black lines indicate that the corresponding genes have been verified as cross-pathway regulatory genes. Two bootstrap values smaller than 70 are indicated in bracket. $V l: V$. longisporum, Vd: V. dahliae, Va: V. albo-atrum, Cg: Colletotrichum graminicola , Ch: C. higginsianum, Ff: Fusarium fujikuroi, Fo: F. oxysporum, Fg: F. graminearum, Pt-r: Pyrenophora tritici-repentis, Lm: Leptosphaeria maculans, Bb: Beauveria bassiana, Ma: Metarhizium anisopliae, Mo: Magnaporthe oryzae, Nc: Neurospora crassa, Sm: Sordaria macrospora, Cp: Cryphonectria parasitica, Pm: Penicillium marneffei, Af: Aspergillus fumigatus, Ani: A. niger, An: A. nidulans, Ca: Candida albicans, Sc: Saccharomyces cerevisiae. (reprinted from Timpner et al. 2013 The Cpc1 regulator of the cross-pathway control of amino acid biosynthesis is required for pathogenicity of the vascular pathogen Verticillium longisporum. Mol Plant Microbe Interact doi.org/10.1094/MPMI-06-13-0181-R) 
Cpc1 from Verticillium species is close to other phytopathogenic fungi like Colletotrichium and Fusarium and further away from Aspergillus or S. cerevisiae. The plant pathogenic fungi can be divided into one group which is close to each other and farer away from the other fungi.

\subsubsection{VICPC1 isogenes were silenced up to $85 \%$ by RNA-mediated gene silencing}

Because two copies of VlCPCl exist gene silencing was used as a method. RNA-mediated gene silencing has been established in several fungi for targeted gene silencing instead of a conventional knockout (Nakayashiki, 2005). A. tumefaciens mediated transformation of $V$. longisporum was applied and thirty independent hygromycin-B-resistant transformants were selected at random for further analysis. To analyze the efficiency of $V l C P C l$ silencing we investigated the mutants by RT-PCR as the silencing of gene expression is the result of posttranscriptional degradation of targeted mRNA. Total RNA was extracted from the VlCPC1sm (VlCPCl silenced mutant) and wild type. RT-PCR results showed the significant knockdown of VlCPC1 transcripts (Fig. 15). The extent of silencing varied in the different transformants between high and moderate levels. The degree of silencing of cross pathway control genes was estimated by RT-PCR by the ratio of the amplification of VlCPCl between the respective transformants and the unsilenced wild type. For each transformant three biological replicates were analyzed. High-frequency silencing was observed for $54 \%$ of the transformants exhibiting reduced gene expression. The extent of silencing varied in the different transformants between high and moderate levels. After 5 generations on SXM the silencing of the VlCPCl isogenes was still stable (Fig. 15). 

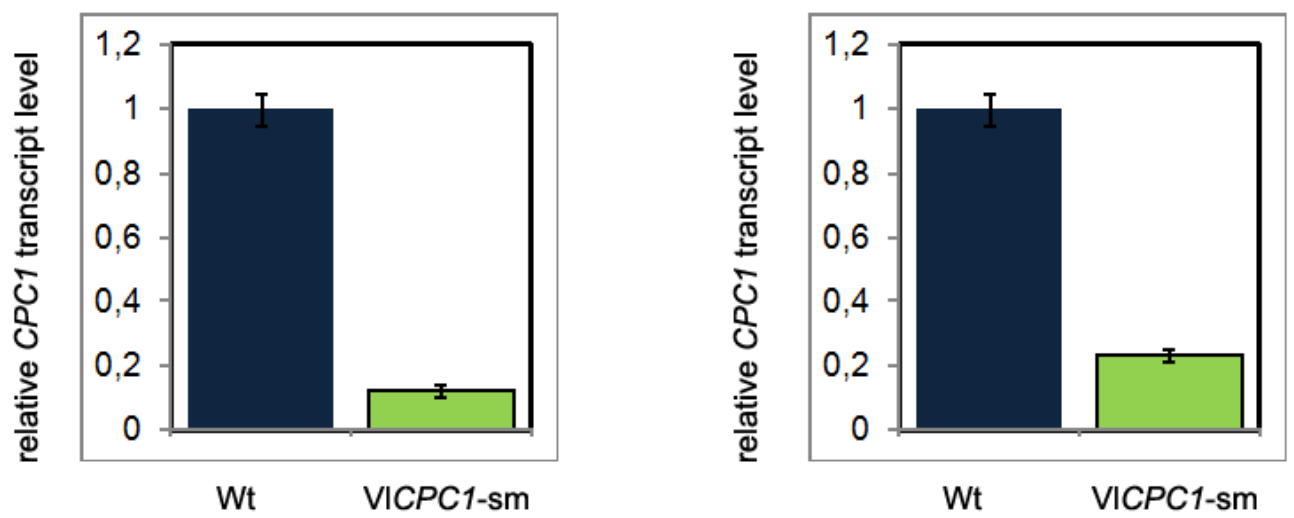

Fig. 15 Expression of cross pathway control encoding genes of $\boldsymbol{V}$. longisporum Relative expression of $V l C P C l$ in $V l 43$ and $V l C P C l \mathrm{sm}$ measured by quantitative real-time PCR. $V l C P C l \mathrm{cDNA}$ was normalized to the histone 2a cDNA. $V l$ 43: wild type, VlCPCl-sm, $C P C l$ silenced mutants. The error bars represent the standard deviation of four different measurements of cDNA. A. Transcript levels of VlCPCl B. Transcript levels of $C P C 1$ after 5 generations on SXM.

\subsubsection{Screening for knockout of $C P C 1$ in $V$. dahliae.}

Silencing strategy is an option for studying genes of interest in fungal species whose genomes are not yet sequenced or for investigating lethal genes. However silencing suppresses maximally about $80-90 \%$ of gene expression in fungi (Nakayashiki et al., 2005; Singh et al., 2011; Singh et al., 2012). The rest activity of the gene (10-20\%) might be still enough for its function in the fungus. To gain deeper insights into the function of CPC1 a deletion of the gene is necessary. Therefore we decided to generate a knockout of CPCl in $V$. dahliae.To analyse mutants and wildtype strain a part of CPC1 gene was amplified via PCR with the primer pair $\mathrm{P} 1 / \mathrm{P} 2$. As a product a 1.3-kb fragment of $C P C 1$ gene from the genome of the wild type strain and a $2.5-\mathrm{kb}$ fragment including the resistance cassette from the genome of the mutants was generated by PCR (Fig. 16). For the identification of correct integration of the deletion construct, candidates from the transformants pool were tested by Southern hybridization. To check for the CPC1 deletion, a probe binding to the 5' region of the gene was used. Genomic DNA isolated from clonal transformants was digested with the enzymes $B c u \mathrm{I}$ and Eco81I, for comparison wild type DNA was used. From Southern hybridisation, the 550-bp fragment of $C P C 1$ gene as probe detected only one band for $C P C 1$ gene in both wild type and mutants (Fig. 16). Two transformants displaying the expected signals at $2.2 \mathrm{~kb}(\mathrm{BcuI}$ and Eco81I) were used for further experiments (Fig. 16). The Southern band from the deletion mutants was $1 \mathrm{~kb}$ bigger than the band from the wild type strain explaining the successful exchange between the deletion cassette and locus of CPCl gene. For final 
validation Southern hybridisation was made with the same probes and enzymes as for the two single knock-outs, and isolates resulting in the correct bands were used for further investigation (Fig. 16).

(a)

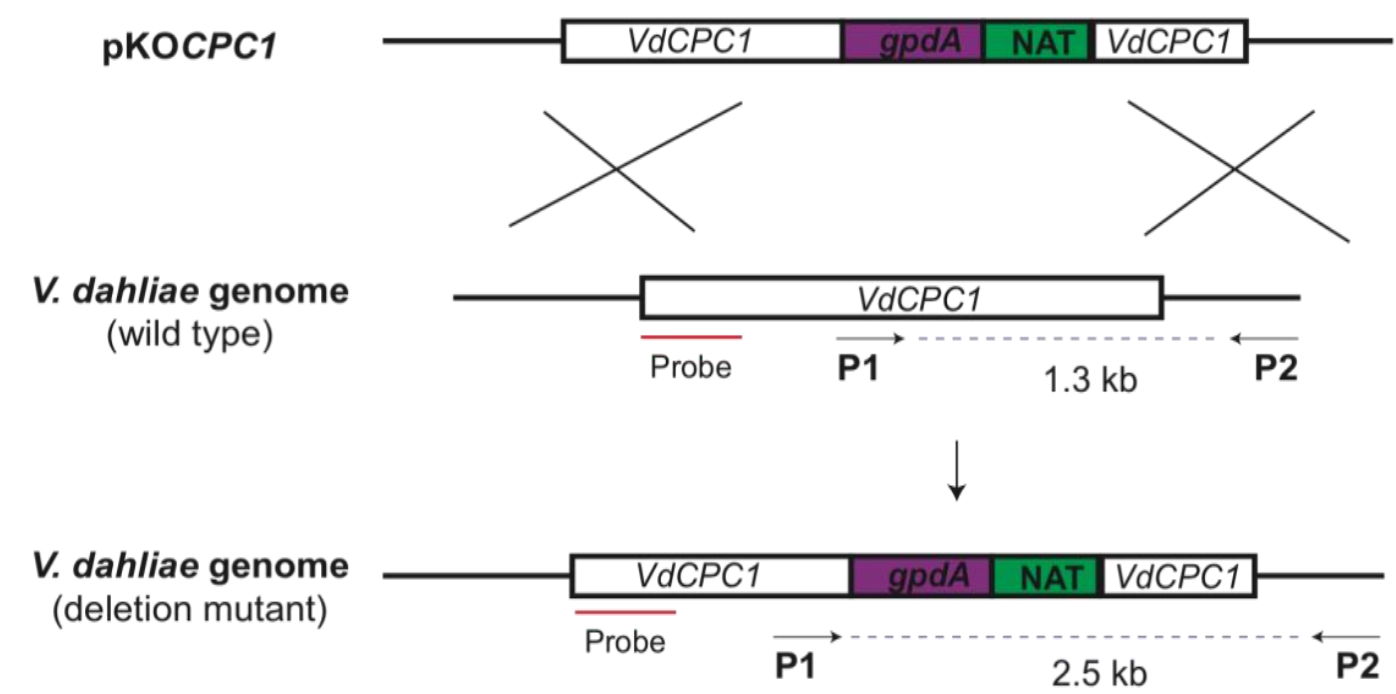

(b)

(c)

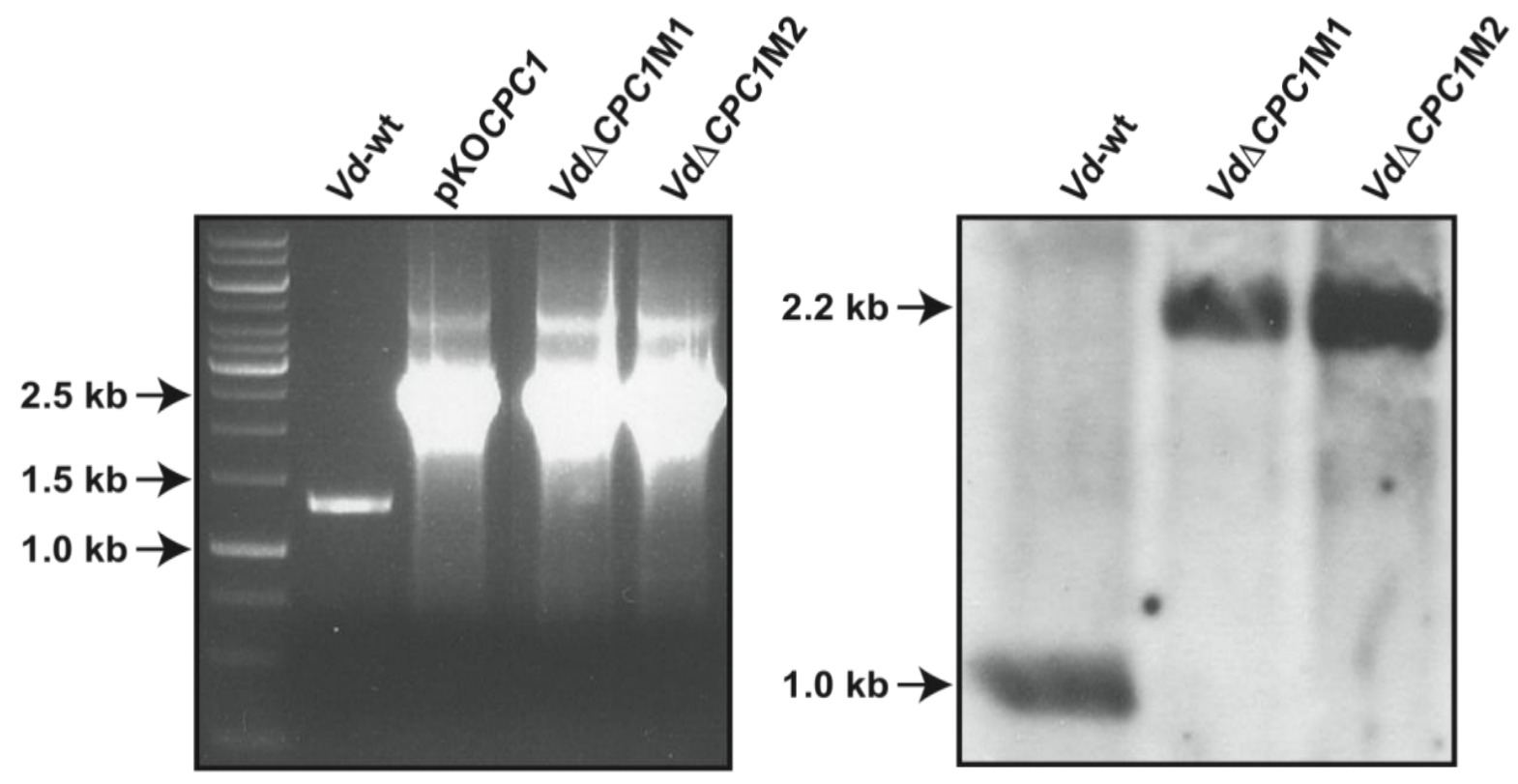

Fig. 16 Strategies for deletion of $C P C 1$ gene in $V$. dahliae and confirmation of the corresponding deletion mutants. A. Model for exchange between the deletion construct and wild type locus of $C P C l$ gene in $V$. dahliae. B. Screening $C P C 1$ deletion mutants using PCR with the primer pair P1/P2. C. Confirmation of the $C P C l$ deletion mutants by Southern hybridization using BcuI and Eco81I for genome digestion. The same band $(2,2 \mathrm{~kb})$ in the mutants is $1 \mathrm{~kb}$ bigger than the one in the wild type VdJR2. 
The plasmid was confirmed via digestion with SalI and XmaJI (Fig. 17). We transformed this deletion construct of CPC1 into V. dahliae strain VdJR2 from tomato. Consequently, 100 transformants were selected randomly.

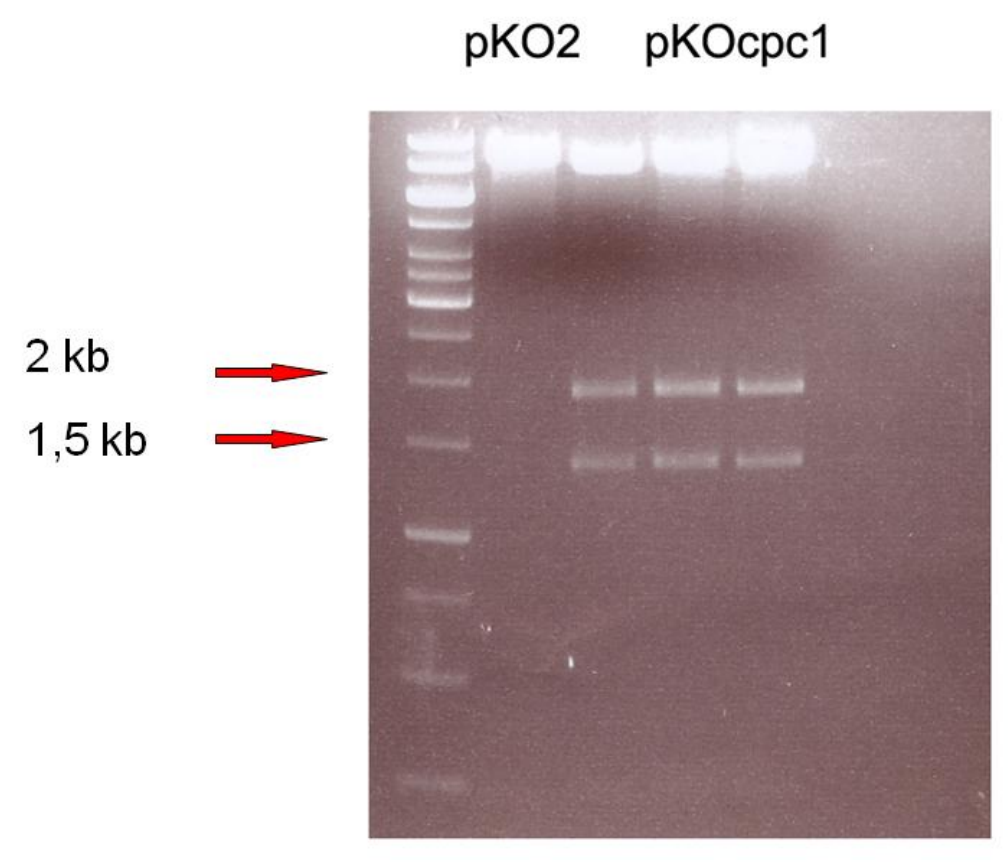

Fig. 17 Control digestion of pKO2 and pKOCPC1. Both plasmids were digested with SalI and XmaJI. For pKOCPCl this resulted in two fragments with the size of $1,5 \mathrm{~kb}$ and $2 \mathrm{~kb}$.

\subsubsection{VICPC1 silenced mutants are strongly reduced in growth under amino acid starvation conditions}

We analyzed the saprotrophic lifestyle of the $V l C P C 1 \mathrm{sm}$ and investigated if silencing of $V l C P C l$ inhibits the growth of $V$. longisporum in the absence of amino acids, One day after inoculation on CDA, $V l C P C l \mathrm{sm}$ could grow like the wild type. For both strains short hyphae were visible and the beginning of mycelial growth (Fig. 18). After 2 days of inoculation on CDA, the mycelial growth increased, and the first conidia could be detected. In addition we studied the effect of $V l C P C l$ silencing on the radial growth of $V$. longisporum. The radial

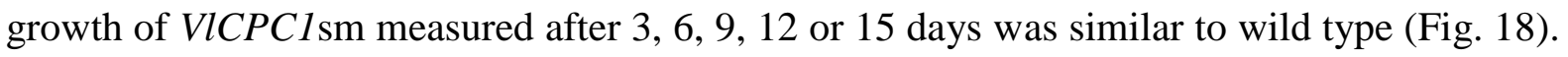
Therefore, growth under normal conditions does not affect a $V l C P C l$ silenced mutant. 
A

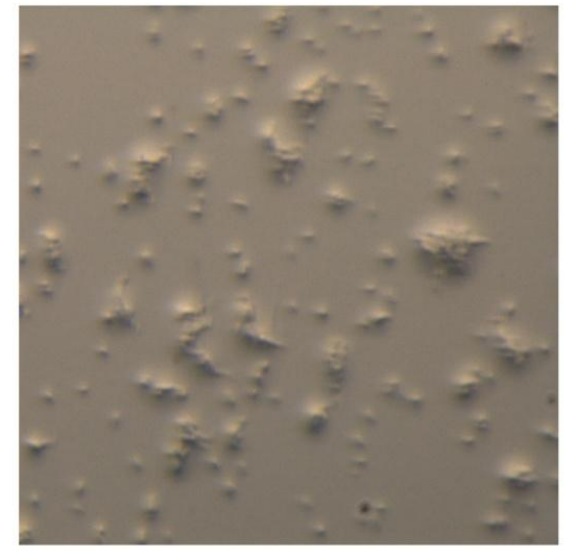

C

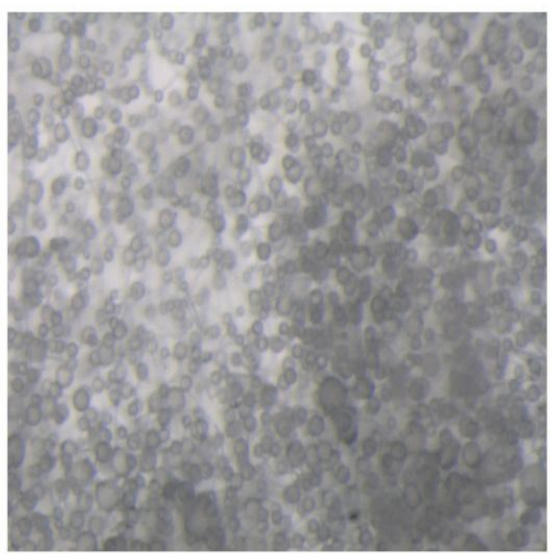

B

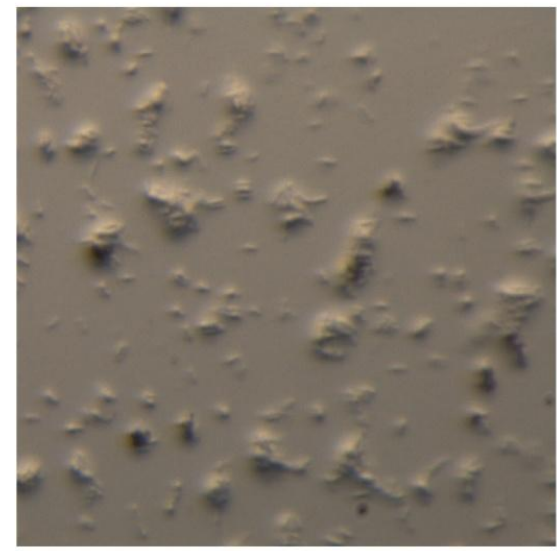

D

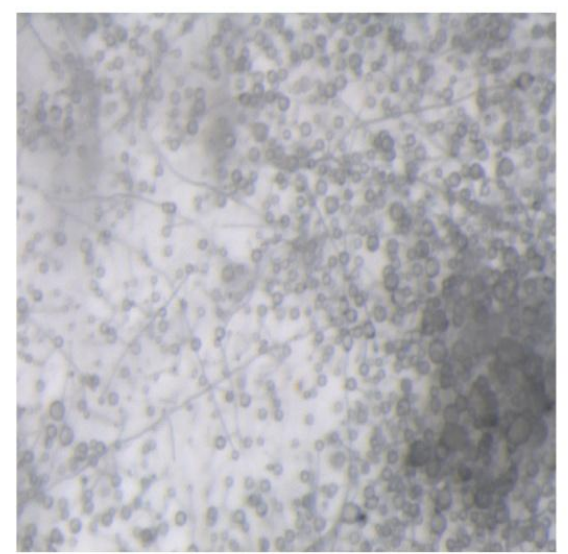

Fig. 18 Light microscopy images of $V$. longisporum wild type (wt) and VlCPC1 silenced mutant (VlCPC1sm). The strains were cultured on CDA as observed at one and 4 days post inoculation. A. and B. $V$. longisporum strain (A) and VlCPClsm (B) after one day of growth. C. and D. V. longisporum strain (C) and $V l C P C 1 \mathrm{sm}(\mathrm{D})$ after four days of growth.

To investigate growth limitations of the mutant in comparison to the wild type we analyzed the effect of the tryptophan analog, 5- MT on VlCPClsm. Addition of 5-MT to CDA causes additional tryptophan starvation. 5-MT acts as false feedback inhibitor of anthranilate synthase, the first enzyme of the tryptophan branch of the pathway(Schürch et al., 1974). Spores of $V l 43$ and $V l C P C l s m$ were plated on CDA supplemented with 5 mM 5-MT (Fig. 19A). The strains were grown for 15 days at $25^{\circ} \mathrm{C}$. One day after inoculation, the wild type formed short hyphae compared to $V l C P C l \mathrm{sm}$ in which only initial germination of conidia could be observed. After 2 days, wild type showed a mycelial net with few conidia, whereas $V l C P C 1 \mathrm{sm}$ formed short hyphae without conidia. Interestingly, the radial growth of $V l C P C 1 \mathrm{sm}$ was significantly lower than in the wild type when the colony diameter was 
determined until 15 days (Fig 19B). It started decreasing gradually at 3 and 6 dpi and decreased up to $40 \%$ at 9 and 12 dpi. Hence, $V l C P C 1$ sm showed severe reduction in initial vegetative growth as well as the growth rate observed under saprotrophic conditions on CDA supplemented with 5-MT. After 3 days of growth the diameter of the silenced mutant is limited to roughly $0,5 \mathrm{~cm}$, whereas the wild type strain has reached approximately $1,5 \mathrm{~cm}$. During the following time points the size of the colonies increases to $1,0 \mathrm{~cm}$ for the mutant and 2,7 cm for wt. However the mutant is never able to compete with the wild type strain under these limiting conditions. The colony diameter is only $1 / 3$ of that of the wild type, whereas plated on CDA no limitation could be observed (Fig. 19A/B).

Deletion of CPCl of $V$. dahliae resulted in wild-type like growth on minimal medium. No differences in growth rate or morphology could be observed. VlCPCl silencing resulted in a wild type like growth. The mutants show a phenotype after treatment with 5-MT, which reduces the growth of these mutants dramatically. Additionally we could demonstrate that 5MT induces expression of $\mathrm{CPCl}$ in mutant and wild type. 5-MT inhibited growth of the CPC1 knockout in $V$. dahliae completely. Growth under non starvation conditions does not affect a VlCPCl silenced mutant. To investigate growth limitations of the mutant in comparison to the wild type we analyzed the effect of the tryptophan analog 5- MT on $V l C P C 1 \mathrm{sm}$. Addition of 5-MT to CDA causes additional tryptophan starvation. 5-MT acts as false feedback inhibitor of anthranilate synthase, the first enzyme of the tryptophan branch of the pathway (Schürch et al. 1974).

Spores of $\mathrm{Vl} 43$ and $V l C P C 1 \mathrm{sm}$ were plated on CDA supplemented with $5 \mathrm{mM}$ 5-MT (Fig. 19). The strains were grown for 15 days at $25^{\circ} \mathrm{C}$. One day after inoculation, the wild type formed short hyphae compared to VlCPClsm in which only initial germination of conidia could be observed (Fig. 18). Next the influence of 5-MT on expression levels of VlCPC1 was examined. Therefore quantitative real-time PCR was performed to determine the expression of $V l C P C l$ in wild type and $V l C P C l \mathrm{sm}$ upon induction with 5-MT (Fig. 19C). CDM was inoculated with spores of $V l 43$ and $V l C P C l \mathrm{sm}$. One culture from each strain served as control, the other cultures were treated with 5-MT for $4 \mathrm{~h}$ and then harvested to extract total RNA of the fungus. Due to the treatment with 5-MT cross pathway control was significantly upregulated in wild type as well as the mutant. But the silenced strain remains at low rates regarding the expression level, in fact even after induction the expression is lesser than in the untreated wt control (Fig. 19C). 
A

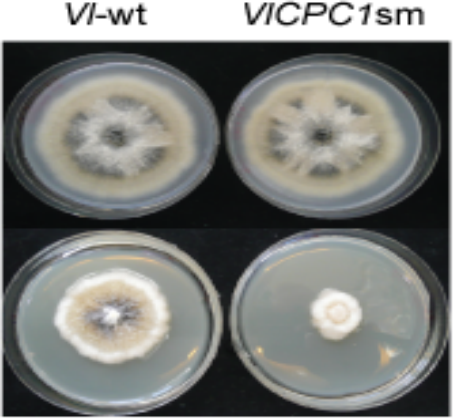

$V d$-wt $\quad V d \triangle C P C 1 \quad C P C 1$ Comp

CDA

DA + 5-MT
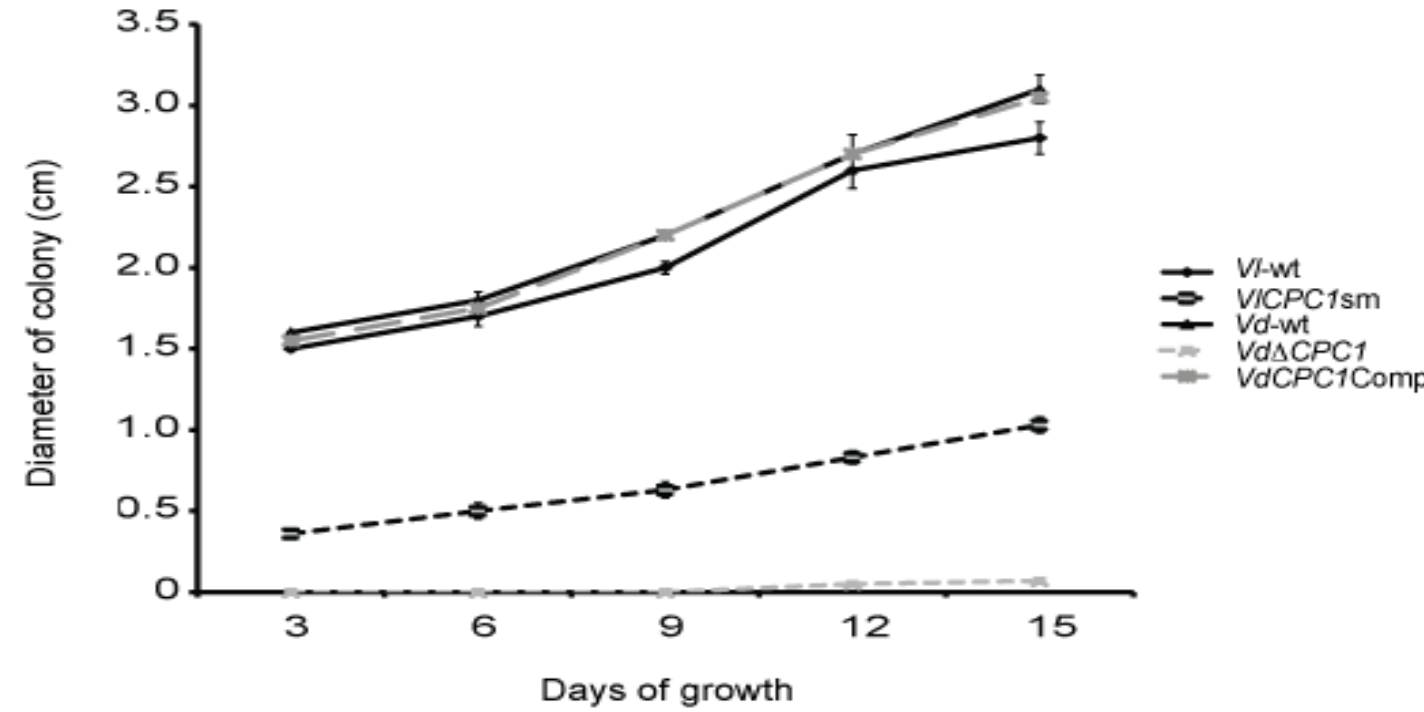

C
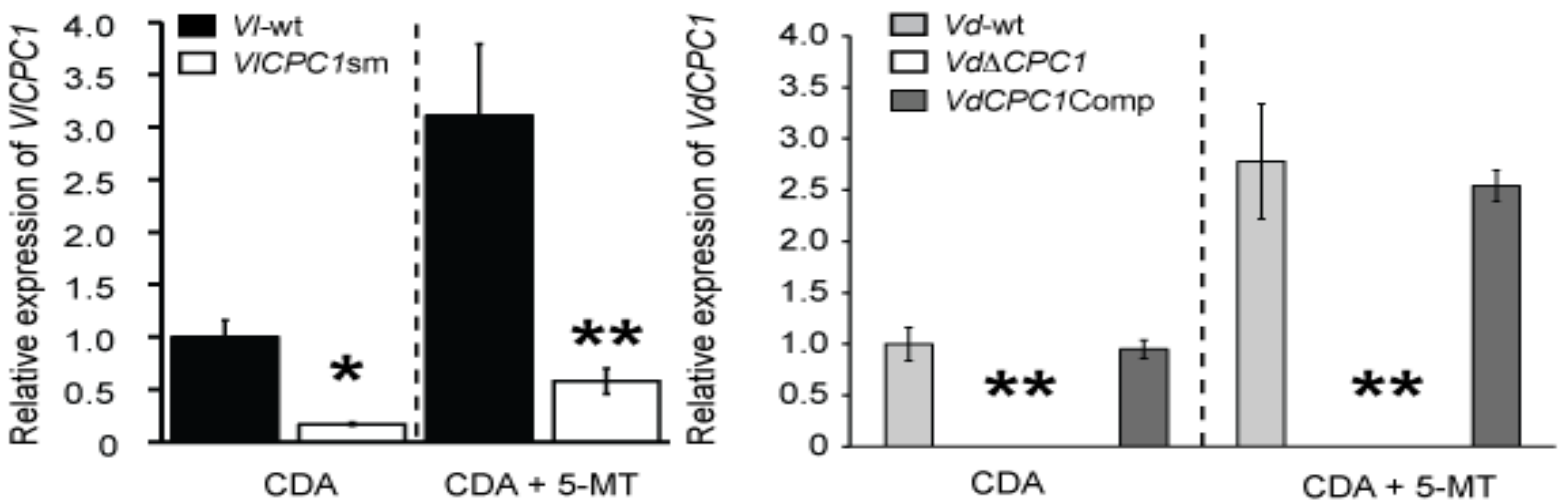

Fig. 19 Influence of 5-methyl-tryptophan (5-MT) on growth of $V$. longisporum and $V$. dahliae. A. Colonies of $V$. longisporum, $V l C P C l$ silenced mutants, $V$. dahliae and $V$. dahliae knockout mutant in the presence or absence of amino acid limitation. Colonies were grown on minimal medium (CDA) after 9 days of inoculation. Amino acid starvation was induced by supplementation with $5 \mathrm{mM}$ 5-MT. B. The $V$. longisporum wild type ( Vlwt) and the silenced mutant $(V l C P C l \mathrm{sm})$ as well as $V$. dahliae wild type $(V d$-wt) and $V$. dahliae knockout mutant $(\mathrm{Vd} \triangle \mathrm{CPCl})$ were plated on CDA containing $5 \mathrm{mM}$ 5-MT. Diameters of colonies were measured at indicated days after inoculation. Error bars represent standard deviation $(\mathrm{n}=5)$. C. Relative expression of $V l C P C l$ and $V d C P C l$ transcripts in the $V$. longisporum wild type and the silenced mutant $V l C P C l$ sm as well as $V$. dahliae wild type and the deletion mutant $V d \triangle C P C l$, respectively under inducing and non-inducing conditions measured by quantitative real-time PCR. The target cDNAs were normalized to $H 2 A$ histone transcript. Significant differences between the wild type and mutants are illustrated by asterisks $(* *$ and * indicate $\mathrm{P} \leq 0.01$ and 0.05 , respectively). (reprinted from Timpner et al. $2013 \mathrm{The} \mathrm{Cpc} 1$ regulator of the crosspathway control of amino acid biosynthesis is required for pathogenicity of the vascular pathogen Verticillium longisporum. Mol Plant Microbe Interact doi.org/10.1094/MPMI-06-13-0181-R) 
We analysed the growth of the $V d \triangle C P C 1$ knockout strain in the absence of plants (Fig. 19). One day after inoculation on CDA, $V d \triangle C P C l$ could grow like the wild type. For both strains short hyphae were visible and the beginning of mycelial growth (data not shown). After 2 days of inoculation on CDA, the mycelial growth increased, and the first conidia could be detected. In addition we studied the effect a deletion might have on the radial growth of $V$. dahliae. The radial growth of $V d \triangle C P C 1$ was measured after 3, 6, 9, 12 or 15 days and was similar to wild type (Fig. 19B). Therefore, growth under normal conditions does not affect a deletion mutant of $C P C 1$. Adding 5-MT to the medium inhibited growth of $\triangle C P C 1$ mutant completely (Fig. 19A/B). While mycelial growth could be observed for $V d$ wt the knockout strain was fully retarded in growth. Even the initial phase with germinating spores could not be investigated. Until the the end of the experiment after 15 days no growth was monitored.

Next the influence of 5 methyl-tryptophan (5-MT) on expression levels of VlCPC1 was examined. Therefore quantitative real-time PCR was performed to determine the expression of $V l C P C 1$ in wild type and $V l C P C l \mathrm{sm}$ upon induction with 5-MT (Fig. 19C). The CDB medium was inoculated with spores of V143 and VlCPClsm. One culture from each strain served as control, the other cultures were treated with 5-MT for $4 \mathrm{~h}$ and then harvested to extract total RNA of the fungus. Due to the treatment with 5-MT cross pathway control was significantly upregulated in wild type as well as the mutant. But the silenced strain remains at low rates regarding the expression level, in fact even after induction the expression is lesser than in the untreated wildtype control (Fig. 19C).

Previous studies analysed the expression of genes involved in amino acid biosynthesis (Schönig et al., 2009). We investigated whether CPC1 of Verticillium might also be involved in certain processes (Fig. 20). The regulation of genes from different amino acid synthesis pathways is controlled by $C P C 1$. While mutant and complementation show significant differences in their expression levels the $C P C 1$ deletion strain did not show any expression of the genes. 
A

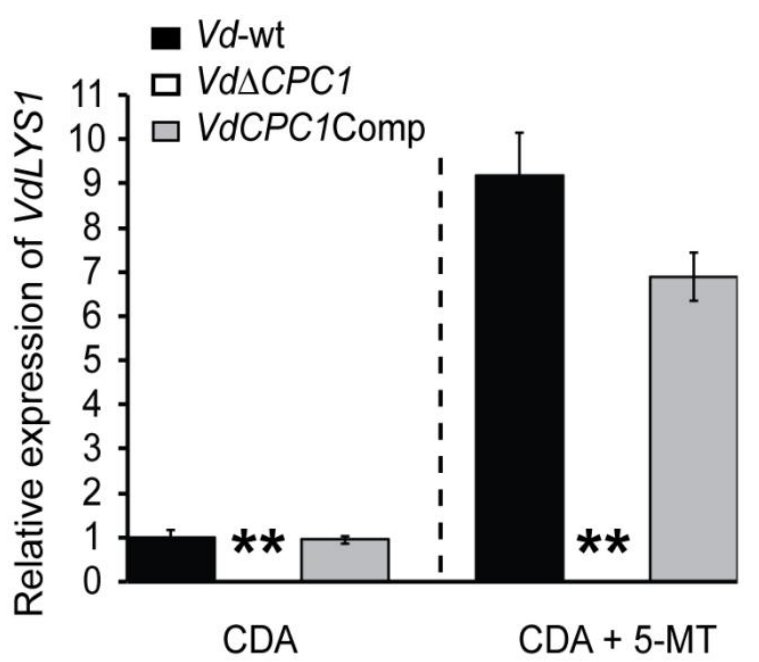

C

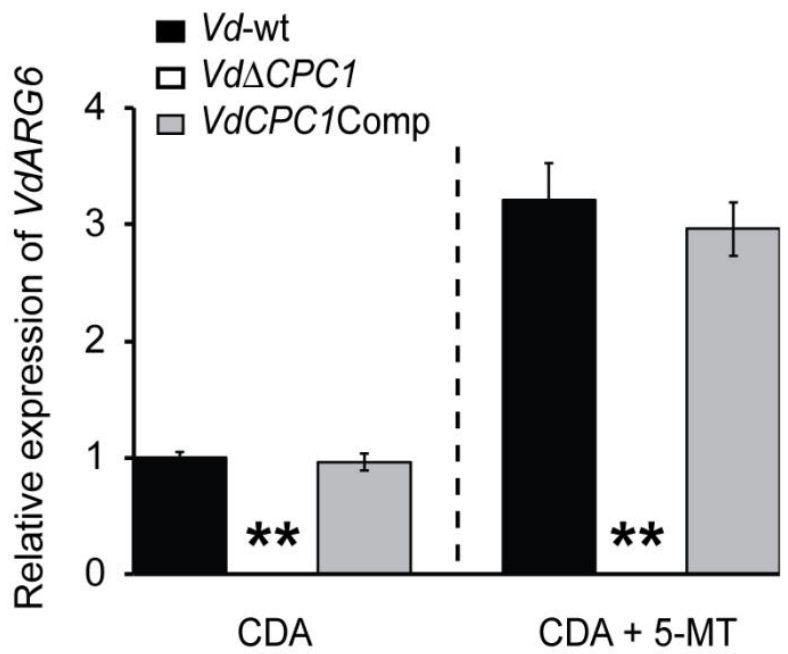

B

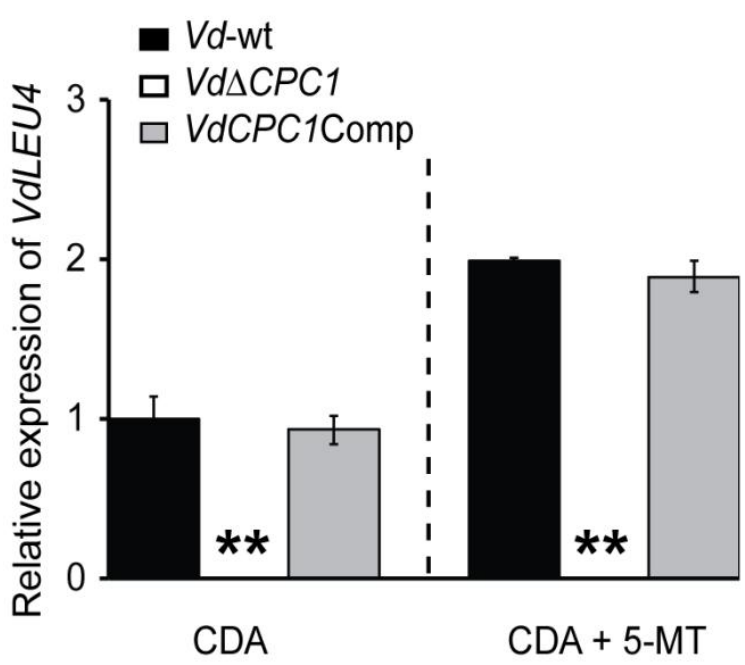

D

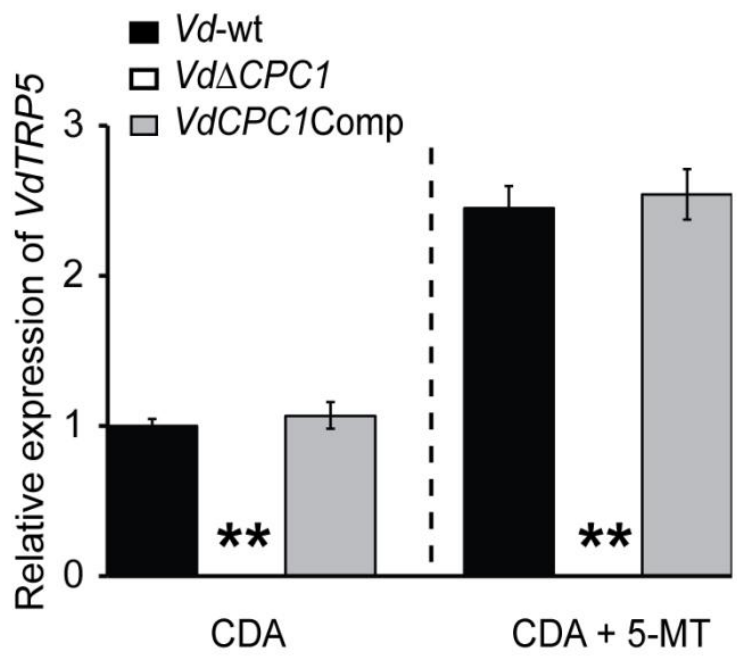

Fig. 20 Comparison of four target genes of amino acid biosynthesis regulated by CPC1 between V. dahliae wild type and the Vd $\triangle$ CPC1 mutant. Relative expression of these targer genes in the fungal wild type, the mutant respectively under inducing and non-inducing conditions measured by quantitative real-time PCR. The target genes of amino acid biosynthesis including VdLYS1 for lysine (A), VdLEU4 for leucine. (B), VdARG6 for arginine (C), and VdTRP5 for tryptophan (D). cDNAs were normalized to H2A histone transcript.. Expression of all four genes is recovered in complementation strain. Significant differences between the wild type and mutants are illustrated by asterisks (** and * indicate $\mathrm{P} \leq 0.01$ and 0.05 , respectively).

\subsubsection{The saprophytic growth of $V d C P C 1$ knockout mutant is not affected.}

In additional experiments the deletion strains of $C P C l$ were tested for growth on different kinds of media. As control and for comparison the wild type strain was used. On minimal medium no obvious growth phenotype was evident (Fig. 19/21). Under stress inducing conditions using the deletion strains grew as well as the wild type (Fig. 19). Compared to amino acid starvation conditions which have a strong effect on mutant growth, treatment with 
these other substances do not influence the growth of $C P C l$ deletion mutant. We analyzed the saprotrophic lifestyle of the $V d C P C 1$ knockout strain (VD $\triangle C P C 1)$ and investigated if a deletion of $V d C P C l$ influences the growth of $V$. dahliae. One day after inoculation on CDA, M1 could grow like the wild type. For both strains short hyphae were visible and the beginning of mycelial growth . After 2 days of inoculation on CDA, the mycelial growth increased, and the first conidia could be detected. In addition we studied the effect a deletion might have on the radial growth of $V$. dahliae. The radial growth of VD $\triangle C P C 1$ was measured after 3, 6, 9, 12 or 15 days and was similar to wild type (Fig. 21). Therefore, growth under normal conditions does not affect a deletion mutant of CPC1.

To confirm inhibiting conditions additional testing of amino acid starvation conditions were performed. Therefore CDA was supplemented with $2 \mathrm{mM}$ methionine sulfoximine (MSX) (Fig. 21). Under amino acid starvation conditions the silenced mutant is strongly inhibited in growth (Fig. 19/21). Additionally different stress inducing agents were added to the medium (Fig. 21). 
A

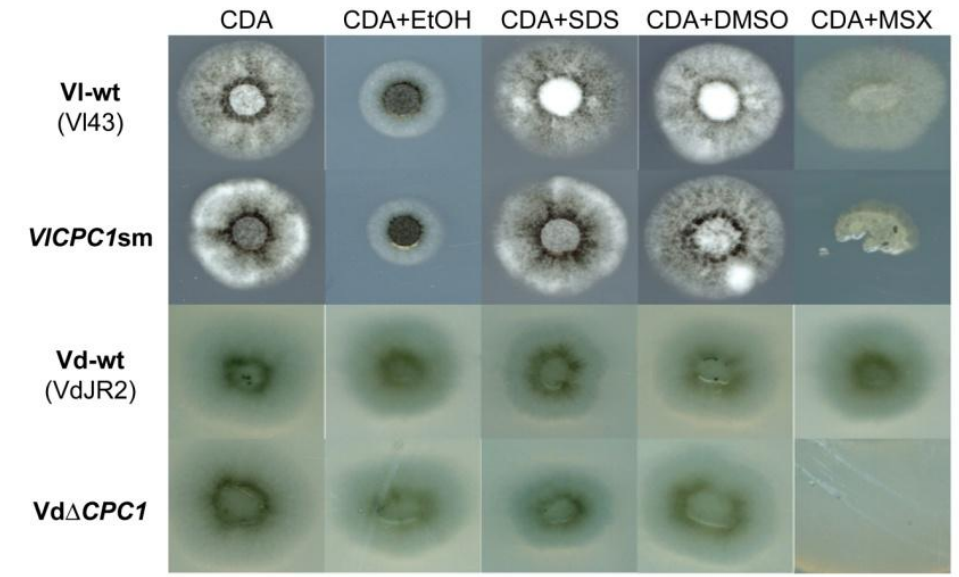

B
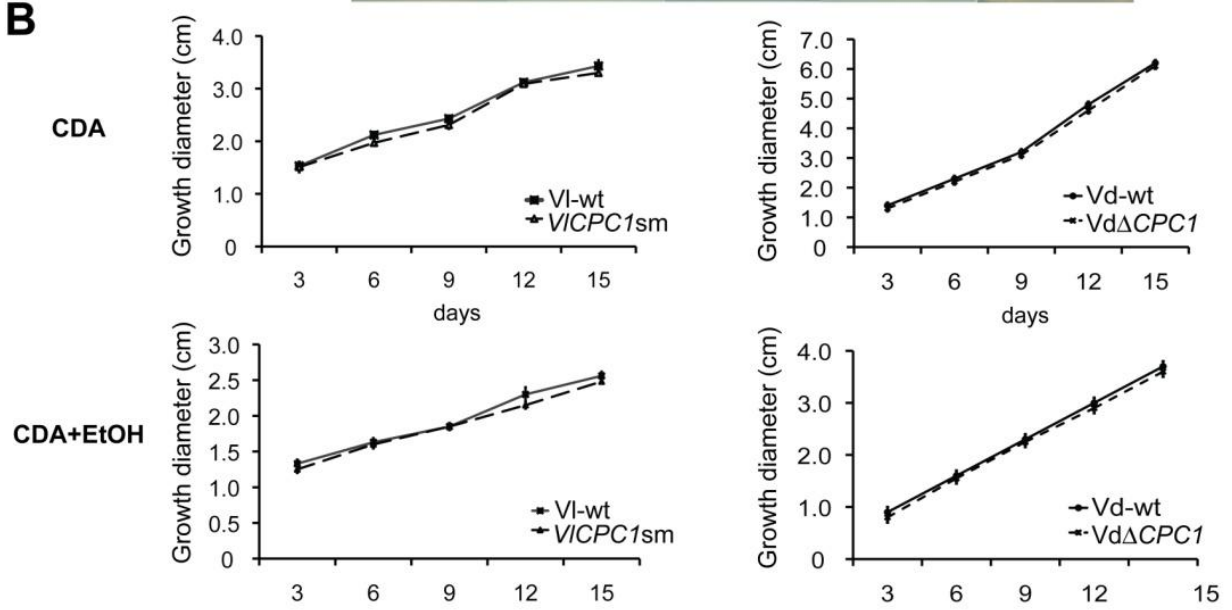

CDA
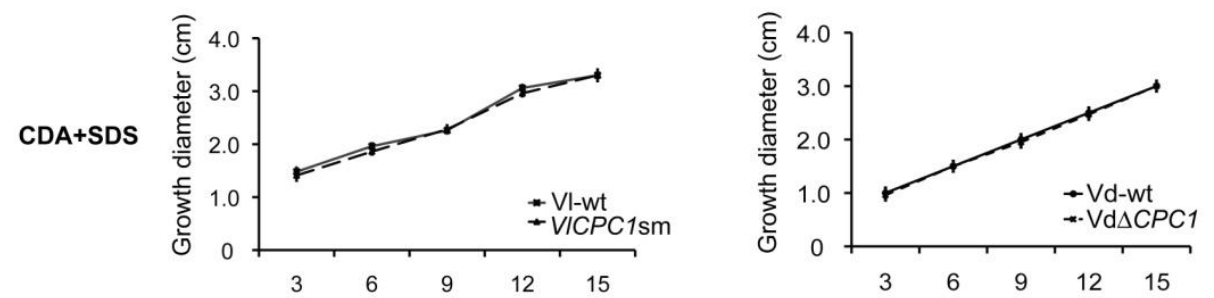

CDA+EtOH
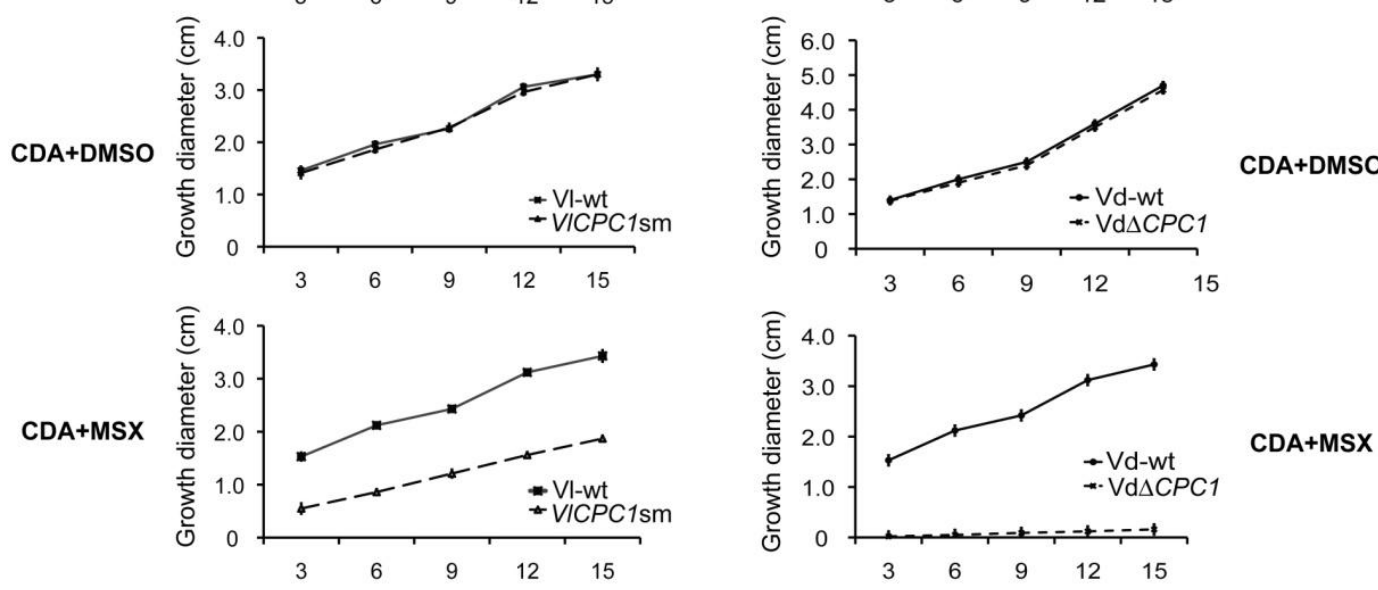

Fig. 21 Influence of different substances on $\boldsymbol{V}$. longisporum growth. A. Images of $V$. longisporum wild type (Vl-wt), $V l C P C l$ silenced mutant $(V l C P C l \mathrm{sm}), V$. dahliae wild type (Vd-wt) and $V d C P C 1$ knockout mutant $(V d \triangle C P C 1)$. Colonies growing on CDA and on CDA supplemented with EtOH (2\%), SDS (0.002\%), DMSO $(0.5 \%)$ or MSX $(2 \mathrm{mM})$ were observed after 9 days of inoculation. B. The diameter of the colonies was measured after 3, 6, 9, 12 and 15 days after inoculation. Error bars represent standard deviation $(n=5)$. 
Furthermore we analyzed whether the number of spores produced by the mutant strain is reduced compared to the wild type (Fig. 22).

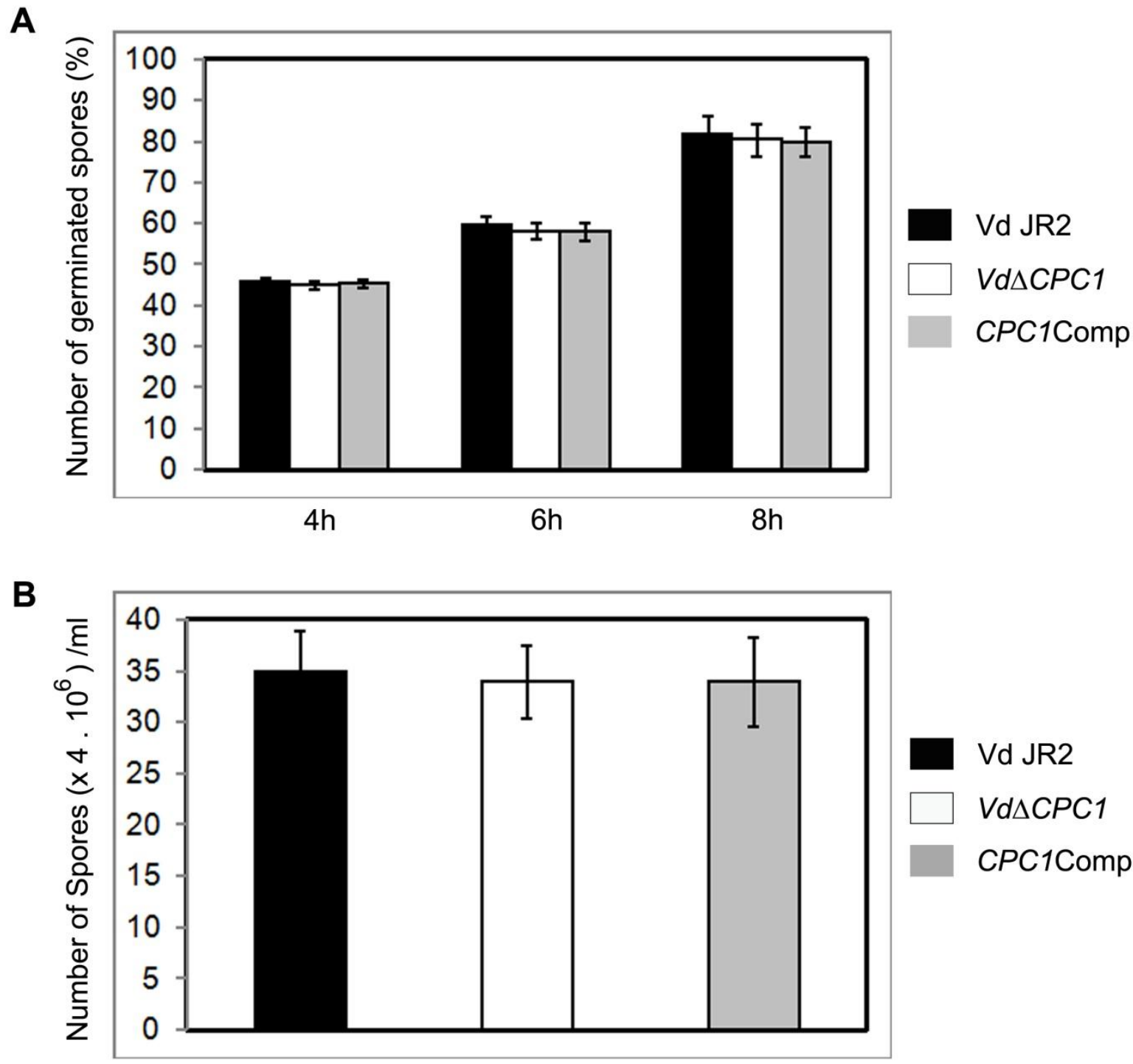

Fig. 22 Comparison of spore levels in WT, CPC1 deletion mutant and complementation mutant. A. percentage of spores germinating after 4,6 and $8 \mathrm{~h}$. B. The mutants show no defect in spore formation on solid as well as in liquid media. This resulted in production of spore level similar to the wild type strain VdJR2. The spore numbers were counted after 8 days.

As we can show a deletion of $C P C l$ does not affect the number of spores generated by the fungus. The capability to reproduce is not affected and therefore does not limit the growth of the fungal mutant.

In addition to plant infection we performed a test on different carbon sources for $V$. dahliae wild type and $\triangle C P C 1$ mutant as well as complementation of $\triangle C P C 1$ (Fig. 23). Therefore we use CDA and exchanged the carbon source of this medium by different carbon sources. The 
mutant is not affected by growth inhibition regarding standard carbon sources like sucrose, glucose, or fructose, but plant derived carbon sources like mannose, lignin, cellulase or xylan affect the growth of the deletion strain. The diameter of the colony is reduced by $30-40 \%$ compared to the wild type. Especially lignin and xylan reduce $\triangle C P C 1$ mutant's growth rate.
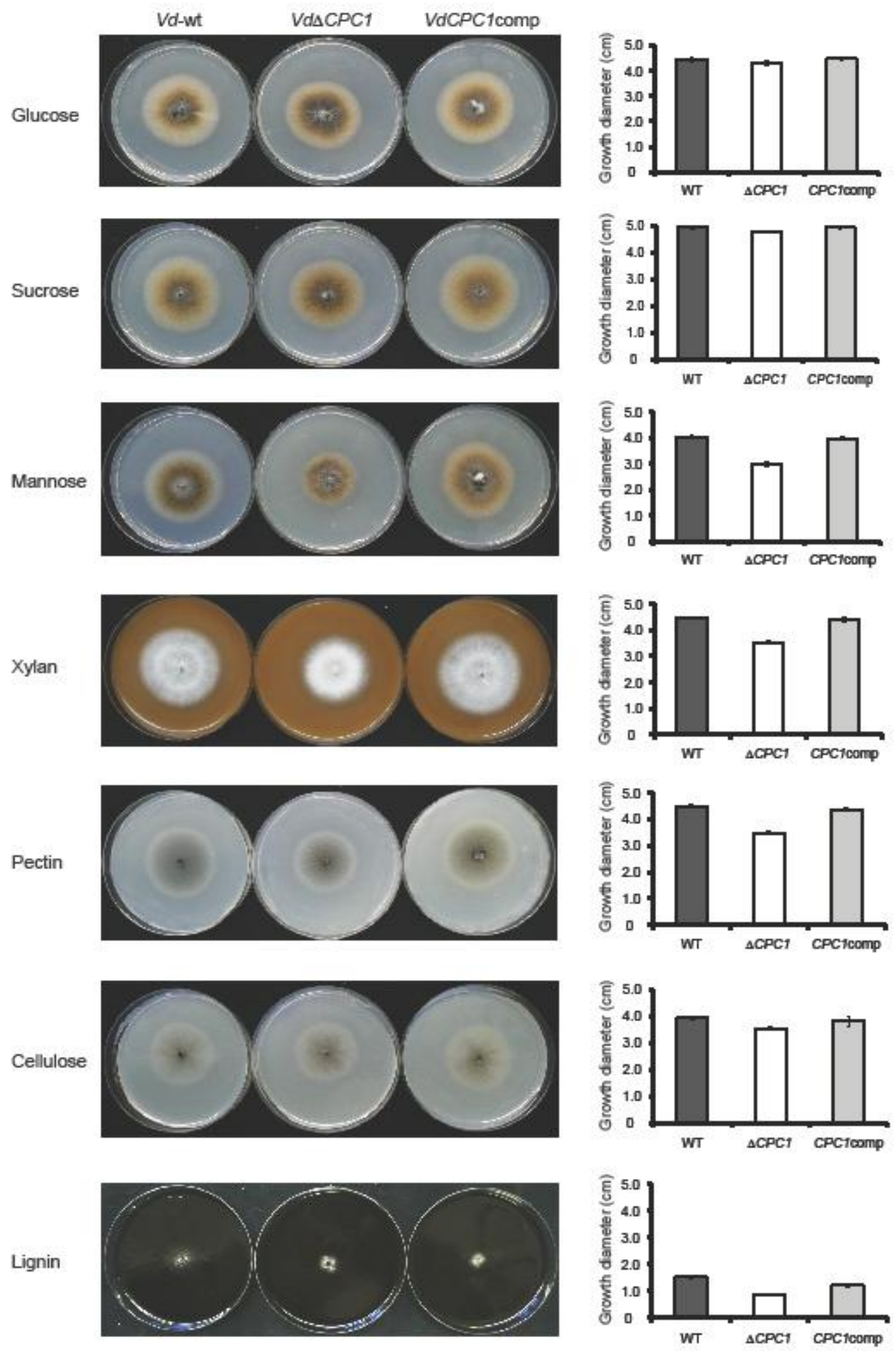
Fig. 23 Growth of the $V$. dahliae wild type $(V d$-wt) or the $V d C P C 1$ deletion mutant $(V d \triangle C P C 1)$ on different carbon sources. CDA medium was used for growth tests with either sucrose or the indicated carbon sources. Diameters of colonies were measured and compared between the wild type and the mutant after ten days of inoculation. (reprinted from Timpner et al. 2013 The Cpc1 regulator of the cross-pathway control of amino acid biosynthesis is required for pathogenicity of the vascular pathogen Verticillium longisporum. Mol Plant Microbe Interact doi.org/10.1094/MPMI-06-13-0181-R)

\subsubsection{Late-stage plant symptoms decrease significantly during infection in the silenced mutant versus the wild type.}

For further knowledge about pathogenicity and disease development in planta, the silenced mutants of $V$. longisporum were investigated when growing inside its host $B$. napus.

To assess the severity of disease, 1-week-old B. napus were infected by root dip inoculation; the height of the plants was measured weekly postinoculation until the plants are highly infected. The plants began to show disease symptoms at $21 \mathrm{dpi}$ and were heavily infected at 35 dpi (Fig. 24).

Two different sets of disease symptoms can be observed during the infection of oilseed rape by $V$. longisporum; the host plant becomes stunted and shows signs of early senescence. Both symptoms are normally visible after 21 days post infection (dpi) and are more distinctive at later time points. One-week-old B. napus seedlings were infected by root dip inoculation to compare the severity of the disease; height and the signs of early senescence (disease scores) of the plants were measured weekly post inoculation until 35 dpi. We analyzed whether $V l C P C 1 \mathrm{sm}$ mutants were still able to colonize B. napus and cause disease, and whether there is also reduced growth of the mutant in the plant or whether the plant is able to inhibit fungal growth. Therefore, the total biomass of $V$. longisporum in B. napus infected with $V l C P C 1 \mathrm{sm}$ versus wild type was analyzed by quantification of the $V$. longisporum DNA in the plant tissue by real-time PCR. The roots hypocotyls, stems and leaves were harvested from the rapeseed plants infected with wild type or $V l C P C 1 \mathrm{sm}$ at various time points.

To investigate the influence of cross pathway control on pathogenicity and disease development inside the plant, the plant infection assay was done by root dip-inoculation of a week old B. napus seedlings. For comparison, B. napus was also infected with $V$. longisporum wild type strain and mock-inoculated with water. The plants were cultivated in a climate chamber and the height of the plants was measured weekly postinoculation until the plants were highly infected. The plants began to show disease symptoms at $21 \mathrm{dpi}$ and were heavily infected at 35 dpi (Fig. 24).

Two different sets of disease symptoms can be observed during the infection of oilseed rape by $V$. longisporum; the host plant becomes stunted and shows signs of early senescence. Both 
symptoms are normally visible after 21 days post infection (dpi) and are more distinctive at later time points. One-week-old B. napus seedlings were infected by root dip inoculation to compare the severity of the disease; height and the signs of early senescence (disease scores) of the plants were measured weekly post inoculation until 35 dpi.

The infected rapeseed plants were scored for disease symptoms by assigning disease scores from 1 to 9 corresponding to asymptomatic to dead plants (Eynck et al., 2007). The plants were observed once a week and in the assessment of the symptoms the yellowing and death of the leaves were considered. The disease scores of the mock-infected plants of more than 1 reflect the yellowing of leaves due to natural senescence. Disease symptoms were visible at $21 \mathrm{dpi}$ and grew more pronounced at $35 \mathrm{dpi}$ (Fig. 24B). Both VlCPClsm and wildtype showed only similar disease scores in the initial phase of the disease at 21 and 28 dpi. At 35 dpi, the disease score of the VICPC1sm-infected plants was lower than the plants infected with wildtype fungus, but still significant in comparison to mock-inoculated control plants (Fig. 24C). This reflects a slowdown of the disease of the plant when infected by the mutant fungi corroborating a reduced impact on senescence. The infected plants did not show any disease symptoms until $14 \mathrm{dpi}$. The disease score of the $V l C P C l \mathrm{sm}$-infected plants was slightly higher (4.23) than the mock-inoculated plants $(3,0)$ but significantly lower than the plants infected with wild type (6.0; Fig. 25). 
A

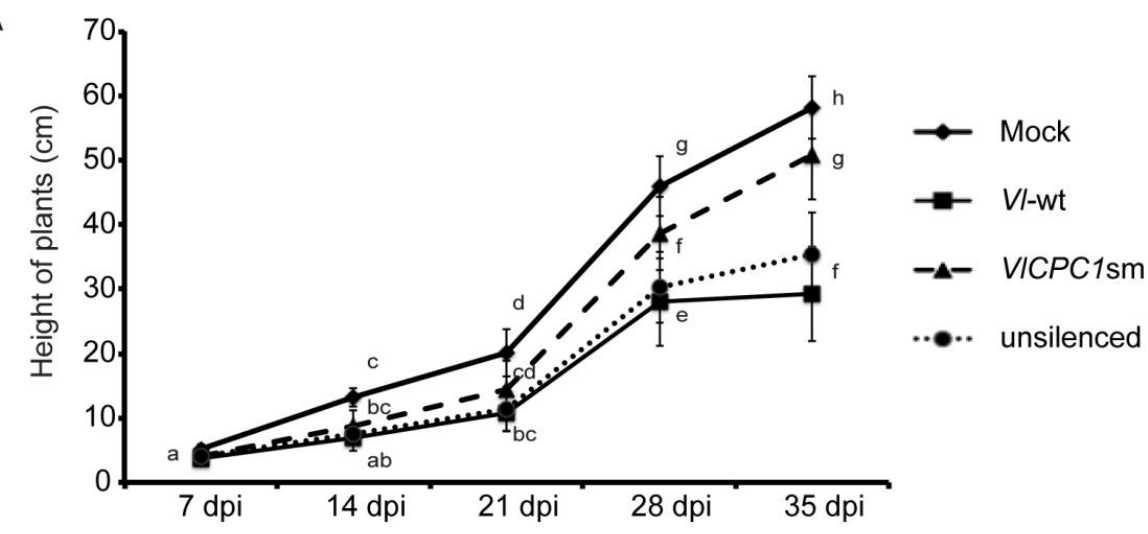

B
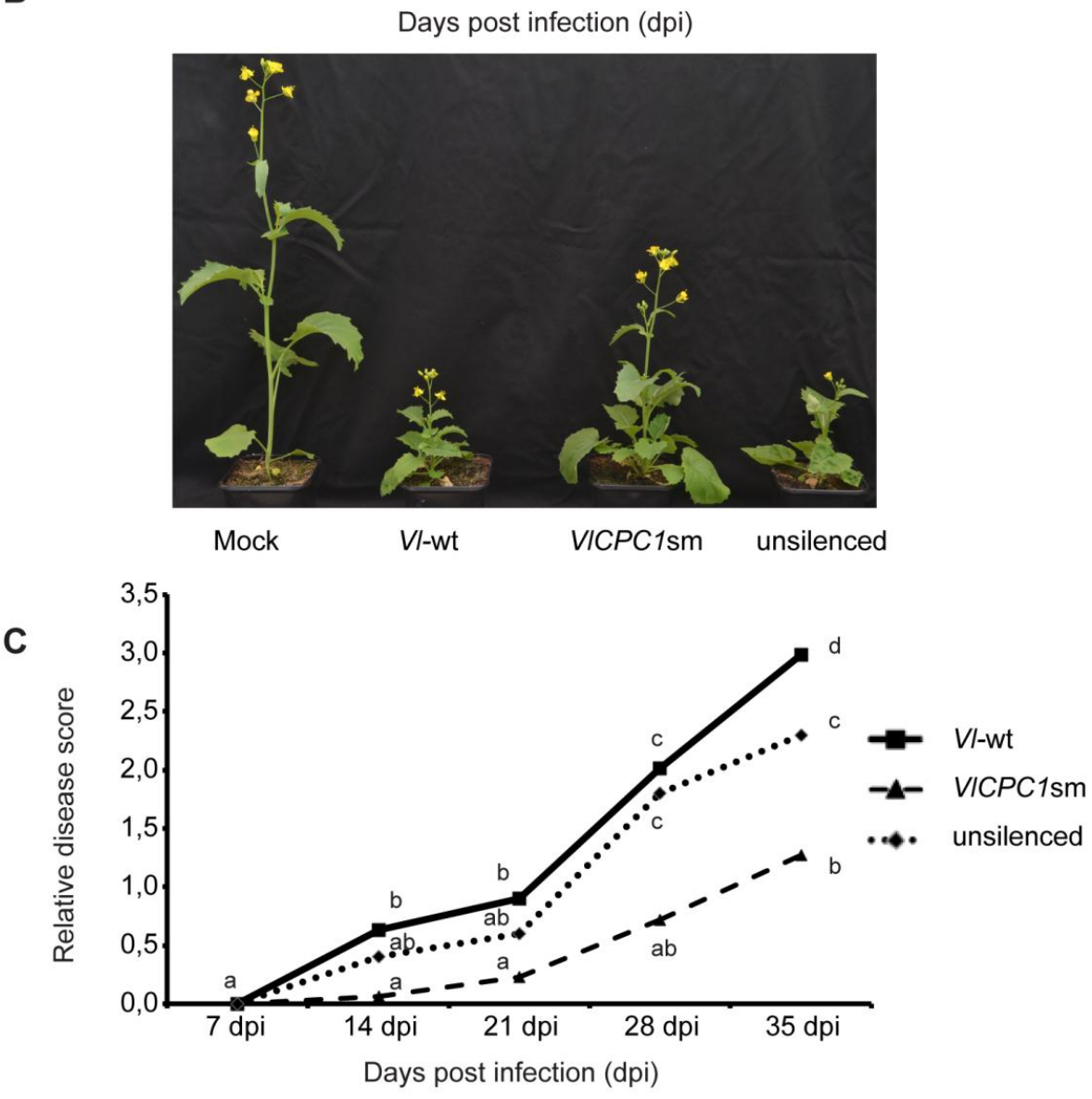

Fig. 24 Assessment of pathogenicity of the VICPC1 silenced mutant. A. Assessment of stunting of rapeseed due to $V$. longisporum infection. The height of 30 replicates each of rapeseed plants infected with wild type (wt) and $V l C P C l$ silenced mutant $(V l C P C l s m)$ was measured at 7, 14, 21, 28, and 35 dpi. For comparison, the height of rapeseed plants mock-inoculated (mock) with tap water was also measured. The plants are heavily infected at 28 and 35 dpi. Data represent average \pm standard deviations of 30 experimental replicates. B. Rapeseed infection assay. Representative B. napus plants shown at $35 \mathrm{dpi}$. C. Assessment of disease development by scoring for disease symptoms according to Eynck et al. (2007). Plants were scored for disease symptoms at 7, 14, 21, 28, and 35 dpi. Data represent average \pm standard deviations of 30 experimental replicates. $V l$ 43, wild type; $V l C P C l$ sm, $V l C P C l$ silenced mutant. The value for mock was detracted from the values for wild type and mutant. Different letters indicate significant differences at $\mathrm{P}<0.05$. (reprinted from Timpner et al. 2013 The $\mathrm{Cpc} 1$ regulator of the cross-pathway control of amino acid biosynthesis is required for pathogenicity of the vascular pathogen Verticillium longisporum. Mol Plant Microbe Interact doi.org/10.1094/MPMI-06-13-0181-R) 


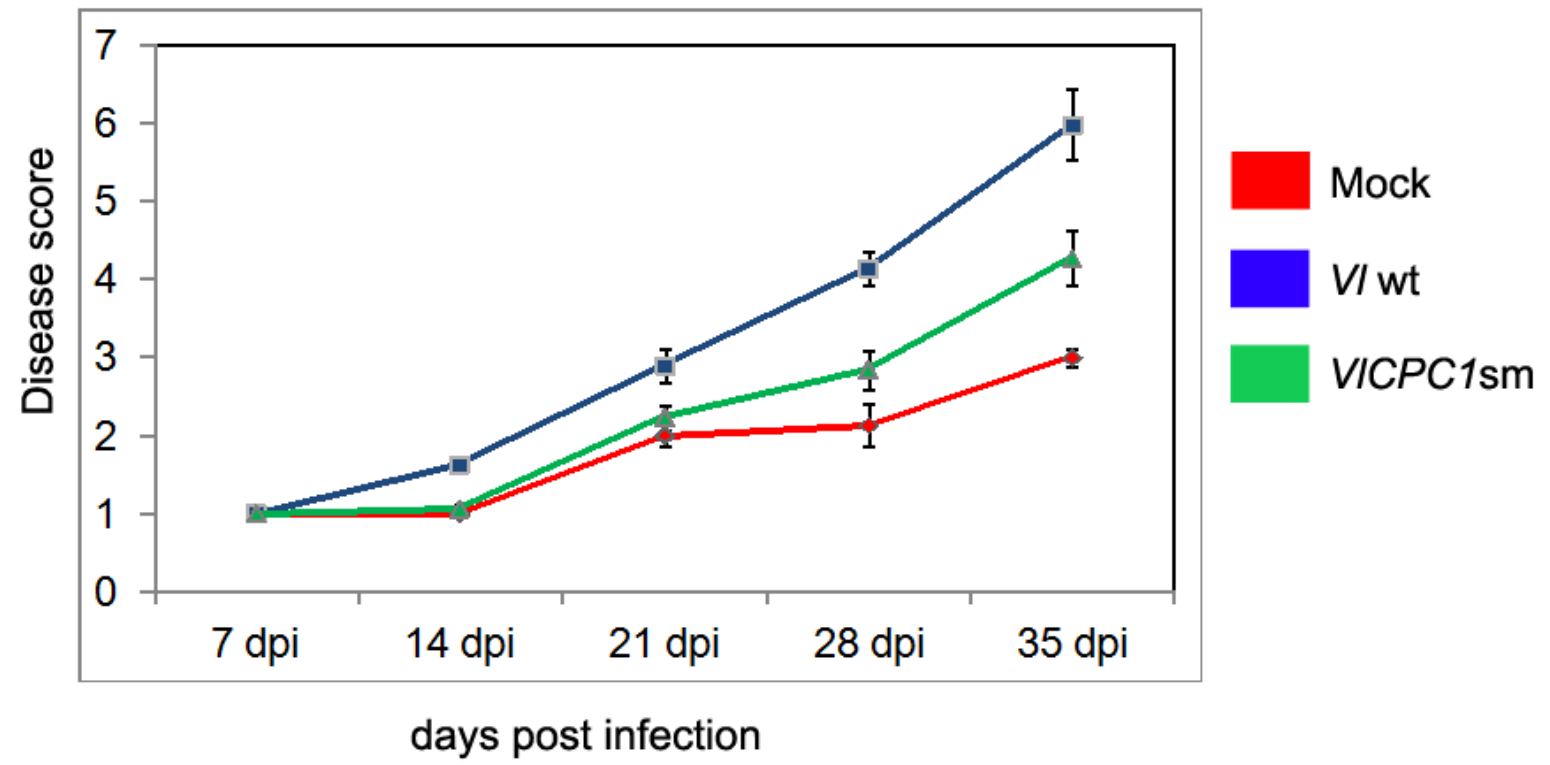

Fig. 25 Assessment of disease development by scoring for disease symptoms according to Eynck $e$ a al. (2007). Plants were scored for disease symptoms at 7, 14, 21, 28, and 35 dpi. Data represent average \pm standard deviations of 30 experimental replicates. $V l 43$, wild type; $V l C P C l \mathrm{sm}, V l C P C l$ silenced mutant.

To investigate whether a deletion of $C P C l$ also affects pathogenicity of $V$. dahliae to its host we performed the infection assays on tomato plants of the $V d C P C l$ deletion mutant (VD $\triangle C P C 1$ ) and the $V$. dahliae wildtype by root-dipping method. The results showed that the mutant is infecting the plants with reduced efficiency whereas the wild type is successfully colonizing its host (Fig. 26). The wild type $V$. dahliae caused the typical symptoms on tomato including stunting and chlorosis (Fig. 26), whereas the $C P C 1$ deletion mutant only exhibited a slight symptom of chlorosis. 


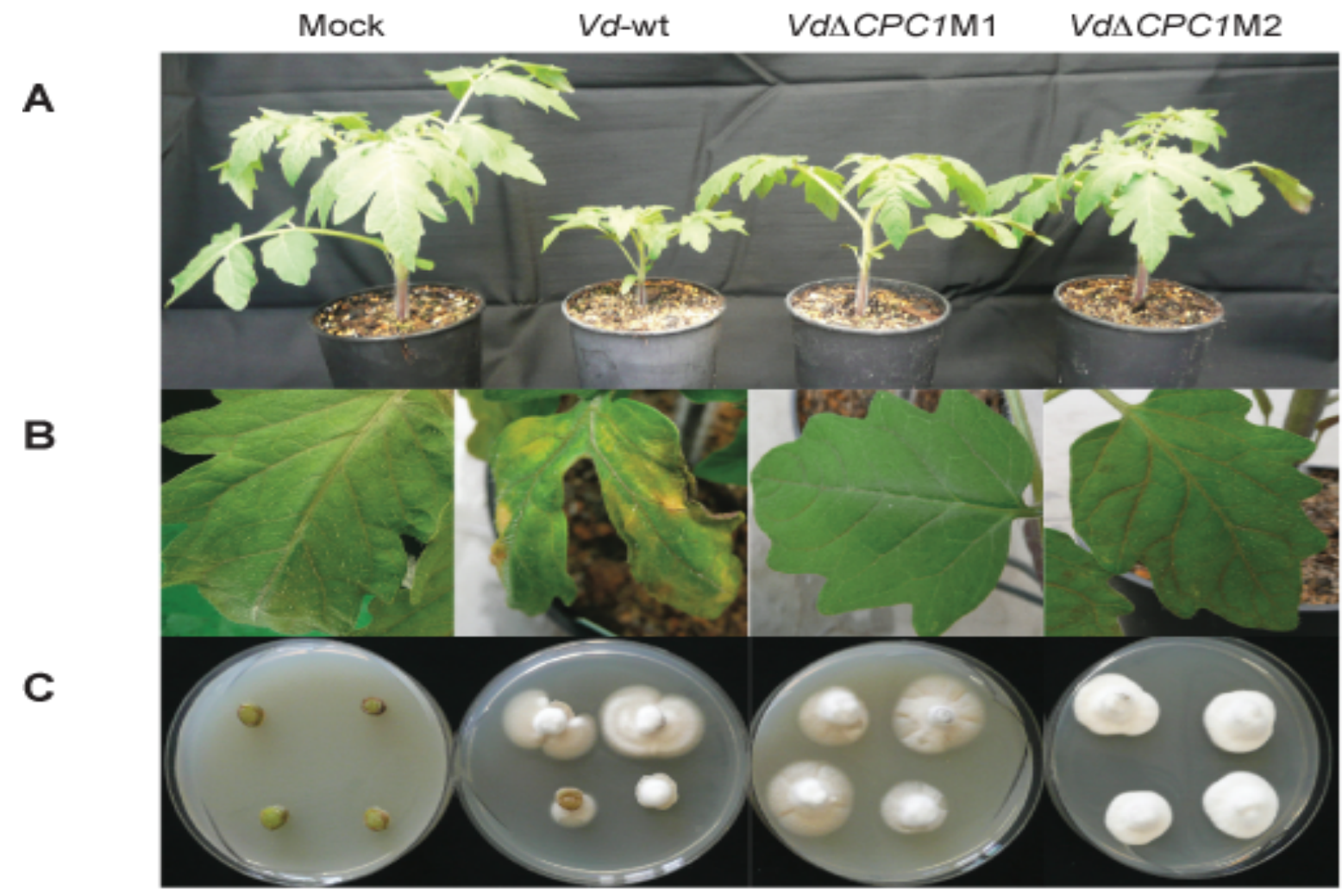

D

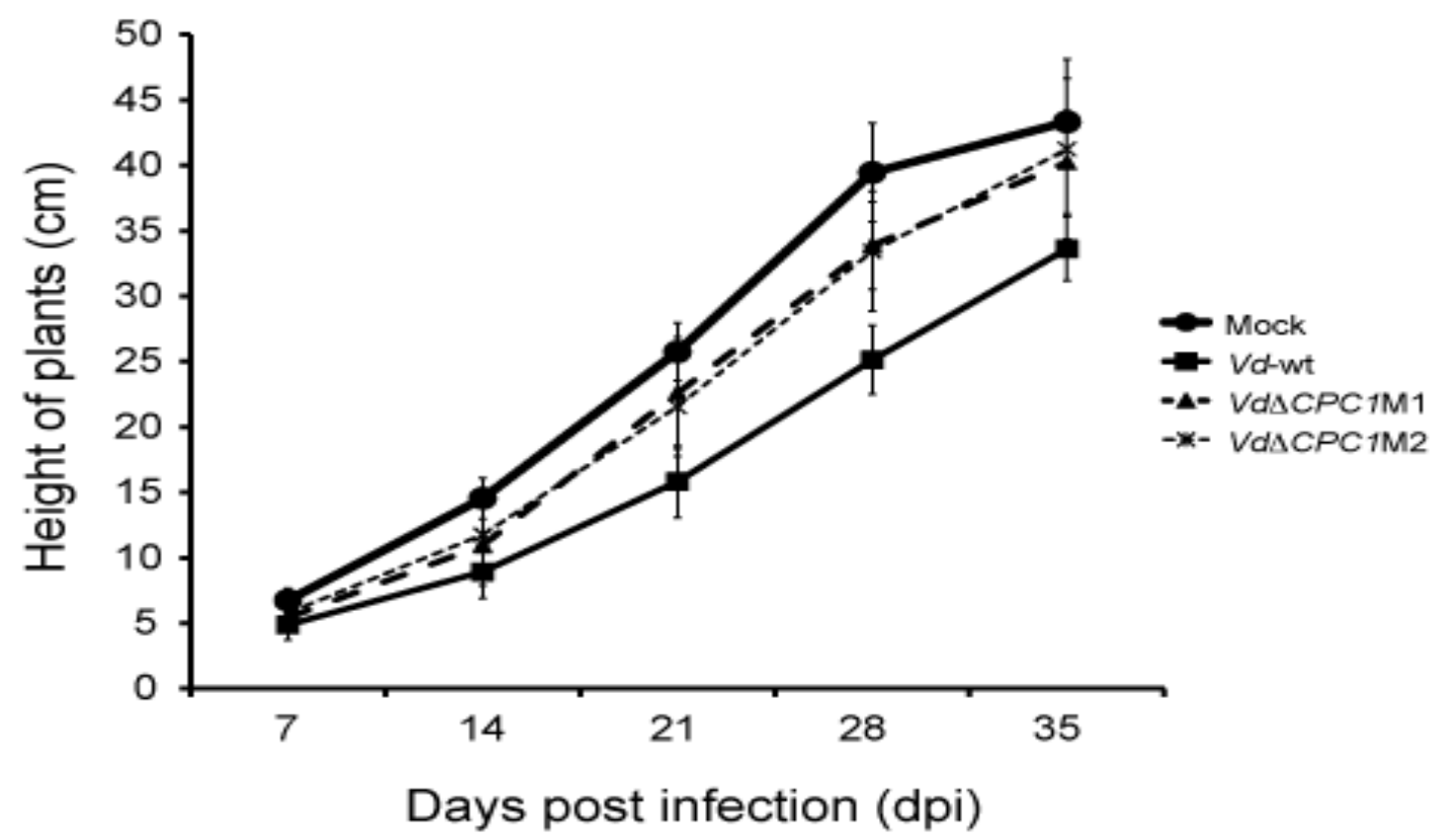

Fig. 26 Assessment of pathogenicity of the VdCPC1 deletion mutant. A. Tomato infection assay. Representative $S$. lycopersicum plants shown at 21 dpi. B. comparison of chlorosis and necrosis symptoms in the leaves of tomato plant. Images of tomato leaves of representative plants from plants infected with $V$. dahliae or $V d C P C 1$ knockout mutant. As control leaves of Mock plants were taken. C. Mutant regrowth test. Image of tomato stem pieces infected with $V$. dahliae or $V d C P C 1$ knockout mutant (VD $\triangle C P C 1$ ) colonies growing PDA plates. As control stem pieces of Mock served as control. D. Assessment of stunting of S. lycopersicum due to $V$. dahliae infection. The height of 20 replicates each of tomato plants was measured.

We analyzed whether $V l C P C l \mathrm{sm}$ mutants were still able to colonize B. napus and cause disease, and whether there is also reduced growth of the mutant in the plant or whether the 
plant is able to inhibit fungal growth. Therefore, the total biomass of $V$. longisporum in B. napus infected with $V l C P C l \mathrm{sm}$ versus wild type was analyzed by quantification of the $V$. longisporum DNA in the plant tissue by real-time PCR (Fig. 27). The roots hypocotyls, stems and leaves were harvested from the rapeseed plants infected with wild type or $V l C P C 1 \mathrm{sm}$ at various time points.

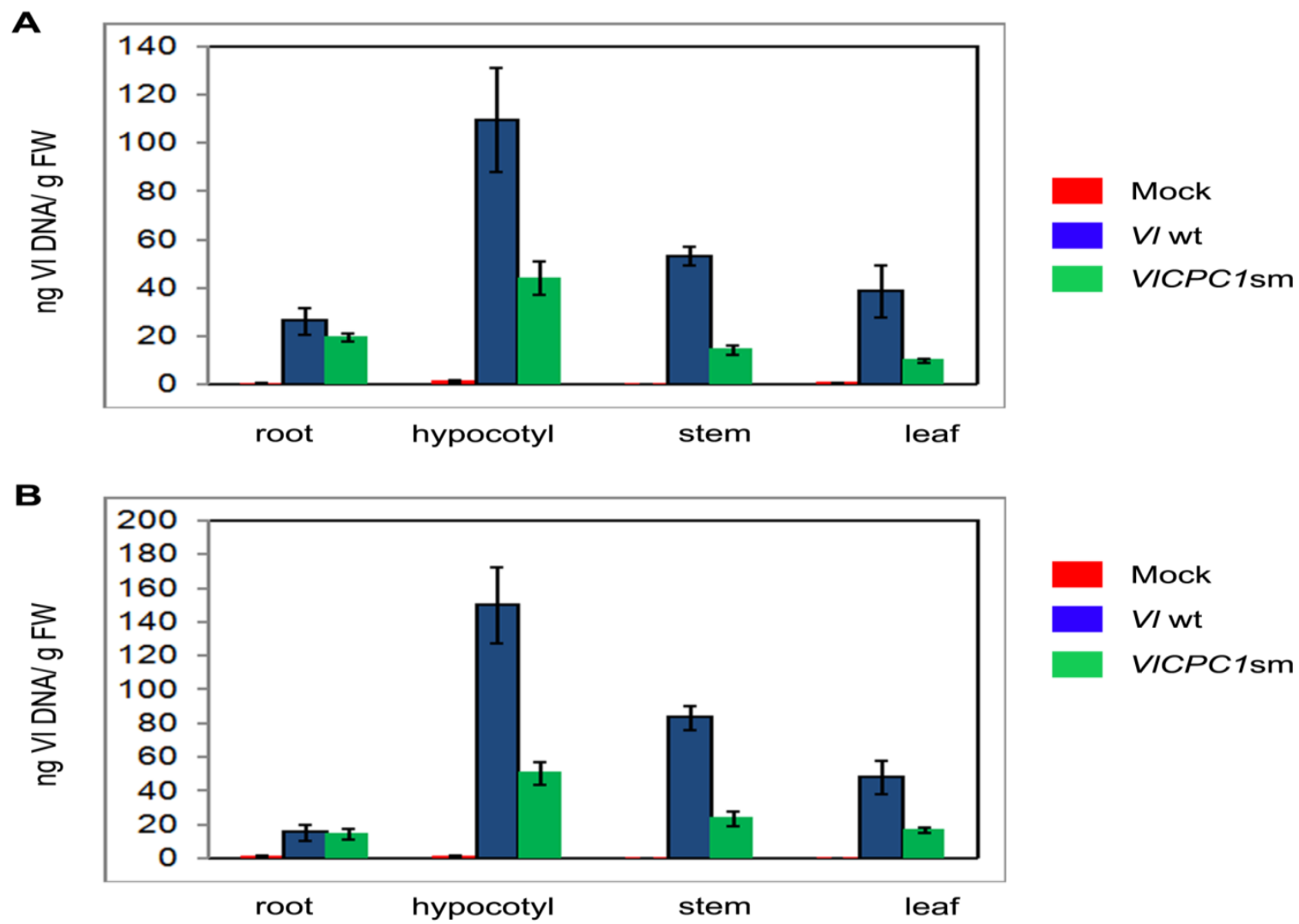

Fig. 27 Infection assay and determination of the $V$. longisporum DNA concentration in infected plant tissues. Different letters indicate significant differences at $P<0.05$. A. V. longisporum DNA concentration in root, hypocotyls, stem and leaves after $28 \mathrm{dpi}$. V. longisporum DNA was measured with real-time PCR in stem, hypocotyls, root and leaves of $B$. napus inoculated with $V l C P C 1$ silenced mutant $(V l C P C 1 \mathrm{sm})$ and wild type (wt) at 35 dpi. Data represent average \pm standard deviations of five experimental replicates. The mockinoculated plants as a control did not show presence of any $V$. longisporum DNA. ng V1 DNA/g FW = nanogram $V$. longisporum DNA/gram fresh weight of plant tissue. B. $V$. longisporum DNA concentration in root, hypocotyls, stem and leaves after 35 dpi. V. longisporum DNA was measured with real-time PCR in stem, hypocotyls, root and leaves of $B$. napus inoculated with $V l C P C l$ silenced mutant $(V l C P C l \mathrm{sm})$ and wild type (wt) at $35 \mathrm{dpi}$. Data represent average \pm standard deviations of five experimental replicates. The mockinoculated plants as a control did not show presence of any $V$. longisporum DNA. ng Vl DNA/g FW = nanogram $V$. longisporum DNA/gram fresh weight of plant tissue.

The DNA content was determined in a time window of $35 \mathrm{dpi}$ where there were no differences within the respective sets of experiments $\left(\mathrm{P}_{(\mathrm{time})}>0.5\right)$. Low concentrations of fungal DNA were found in roots, they did not vary between $V l 43$, VIcpc $1 \mathrm{sm}$ infected plants, but show a difference towards the mock inoculated plants (Fig. 27). However, in the 
hypocotyls where the highest concentration of fungal DNA (100-150 ng/g FW) was present, peculiar differences between plants infected with wild type strain Vl43 or the silenced strains $V l C P C 1 \mathrm{sm}$ (Fig. 27) could be investigated the mutant's amount did not increase from $28 \mathrm{dpi}$ (Fig. 27A) to 35 dpi like the wild type, in fact it does not change, while the wild type amount increases from 100 to nearly 150 ng/g FW. Fungal DNA lower in stems (40-75 ng/g FW) than in hypocotyl (Fig. 27). These data suggest that the mutant strain is able to perform the initial colonization of the plant but has some difficulties in the hypocotyl of the plant (Fig. 27).

After 35 days post infection (dpi) in planta, the silencing of the VlCPCl isogenes was still stable (Fig. 28).

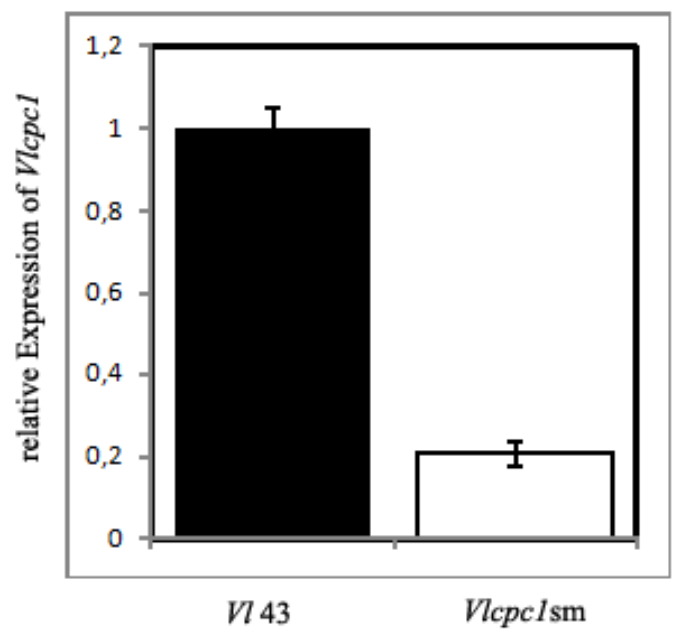

Fig. 28 Expression of cross pathway control encoding genes of $\boldsymbol{V}$. longisporum. Relative expression of VlCPC1 in B. napus measured by quantitative real-time PCR. VlCPC1 cDNA was normalized to the histone 2a cDNA. Relative expression of $V l C P C l$ in $V l 43$ and $V l C P C l \mathrm{sm}$ measured by quantitative real-time PCR. $V l C P C l$ cDNA was normalized to the histone cDNA. $V l 43$ : wild type, $V l C P C l$-sm, $c p c l$ silenced mutants. The error bars represent the standard deviation of four different measurements of the same cDNA. cDNA from 35 days old host plants mock-inoculated with water served as control. $V l 43$, cDNA from $B$. napus infected with wild type, $V l C P C l$-sm, cDNA from B. napus infected with VlCPCl silenced mutant. The error bars represent the standard deviation of triplicates.

\subsection{7. $V d C P C 1$ knockout leads to reduced pathogenicity of the fungus.}

We performed the infection assays of the $V d C P C 1$ deletion mutant $(V d \triangle C P C 1)$ and the wild type strain VdJR2 on tomato plants by root-dipping method. The results showed that the mutant is infecting the plants with reduced efficiency while the wild type is successfully colonizing its host (Fig. 29). The wild type VdJR2 caused the typical symptoms on tomato 
including stunting and chlorosis, whereas the $C P C 1$ deletion mutant only exhibited a slight symptom of chlorosis. This suggests that $C P C 1$ gene is involved in virulence of the fungus.

It was also possibly to regrow the strains out of the infected plants. Therefore pieces of the stem were sterilized and plated on PDA plates. After five days the growth of the mutants was obvious.

For $\mathrm{Vd} \triangle C P C 1$ the amount of fungal DNA in the tomato hypocotyls is also reduced (Fig. 29). Compared to V. longisporum in B. napus the amount of DNA is lower and the difference between wild type and mutant is less prominent, but the DNA concentration is about $40 \%$ lower than in $V$. dahliae wild type.

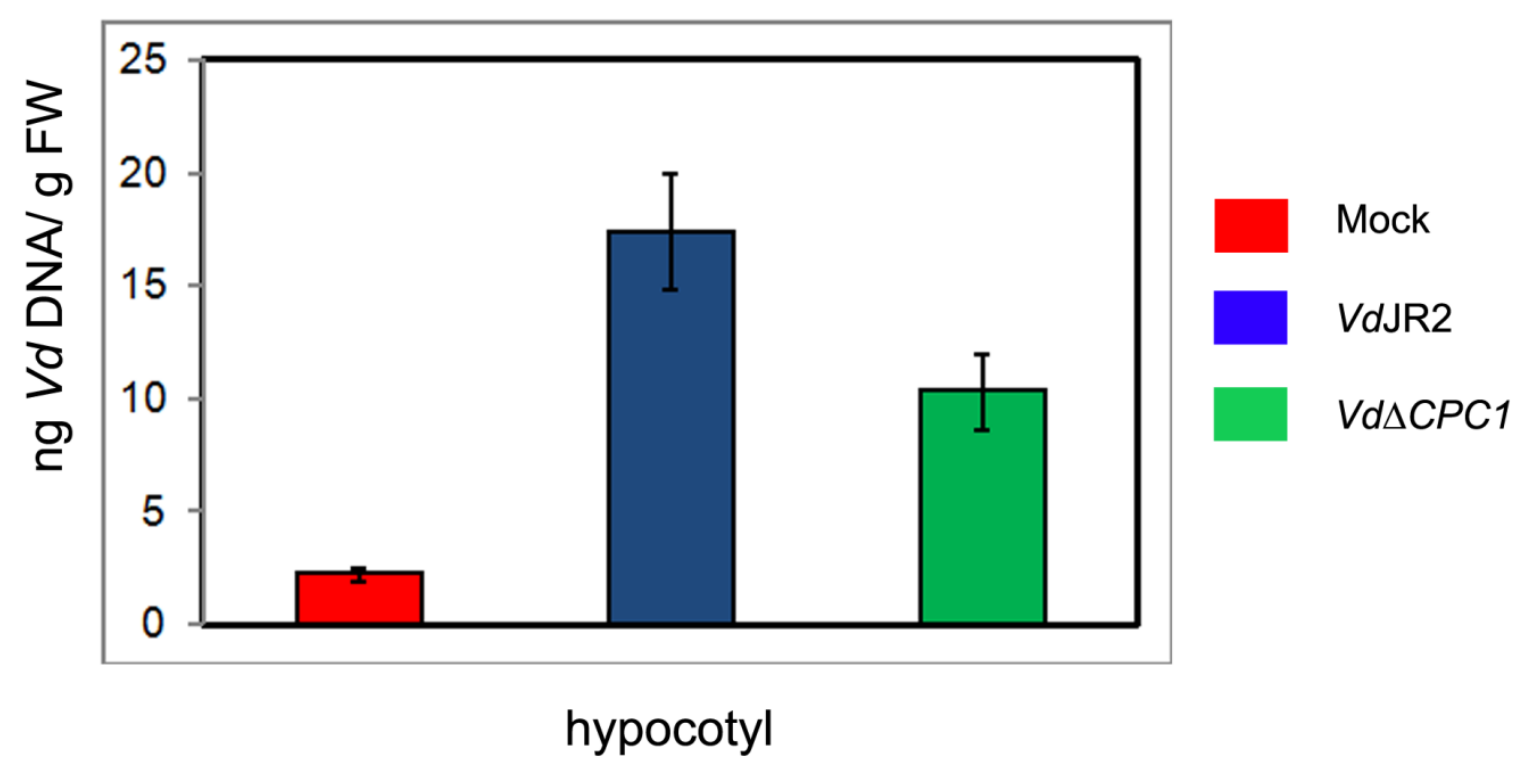

Fig. 29 Infection assay and determination of the $V$. dahliae DNA concentration in infected plant tissues. $V$. dahliae DNA concentration in hypocotyls, after 35 dpi. $V$. dahliae DNA was measured with real-time PCR in hypocotyls, of S. lycopersicum inoculated with $V d C P C 1$ knockout srain $(V d \triangle C P C 1)$ and wild type (wt) at 35 dpi. Data represent average \pm standard deviations of five experimental replicates. The mock-inoculated plants as a control did not show presence of any $V$. dahliae DNA. ng Vd DNA/g FW = nanogram $V$. dahliae DNA/gram fresh weight of plant tissue.

To confirm whether a deletion of $C P C l$ is responsible for reduced pathogenicity of the fungus, a complementation of the $\mathrm{Vd} \triangle C P C 1$ mutant was tested for plant infection together with $\mathrm{Vd} \triangle C P C 1$ and Vd-wt (Fig. 30). 
A

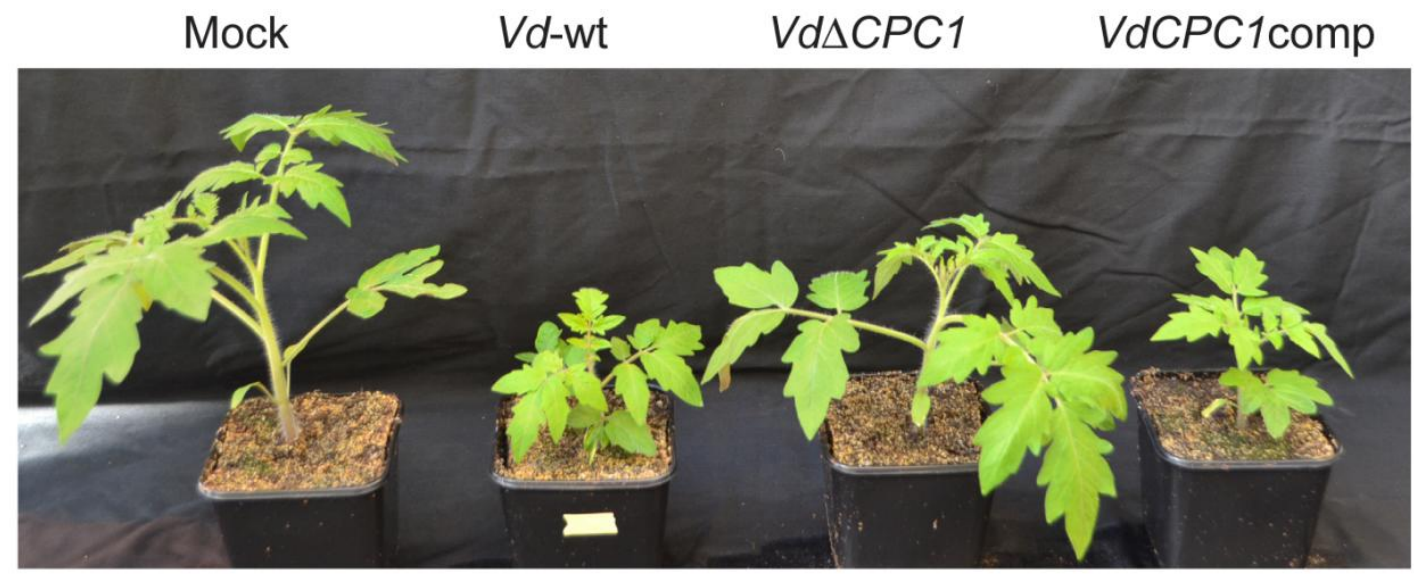

B
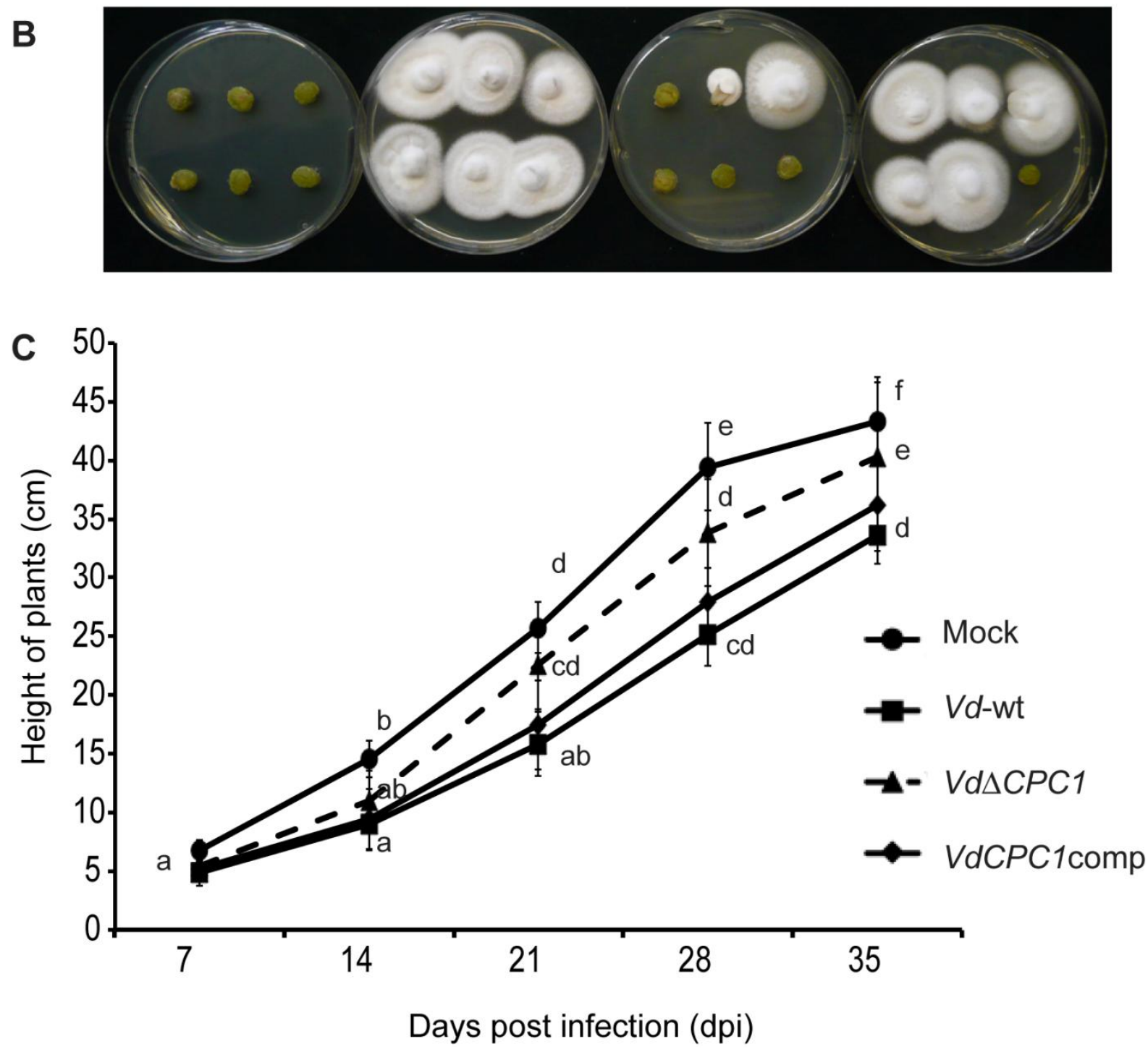

Fig. 30 Assessment of pathogenicity of the VdCPC1 deletion mutant an complementation. A. Tomato infection assay. Representative $S$. lycopersicum plants shown at $21 \mathrm{dpi}$. B. Mutant regrowth test. Image of tomato stem pieces infected with $V$. dahliae, $V d C P C 1$ knockout mutant $(\mathrm{Vd} \triangle C P C 1)$ or $V d C P C 1$ complementation mutant (VdCPClcomp) colonies growing PDA plates. As control stem pieces of Mock served as control.. C. Assessment of stunting of S. lycopersicum due to V. dahliae infection. The height of 20 replicates each of tomato plants Different letters indicate significant differences at $\mathrm{P}<0.05$. (reprinted from Timpner et al. 2013 The Cpc1 regulator of the cross-pathway control of amino acid biosynthesis is required for pathogenicity of the vascular pathogen Verticillium longisporum. Mol Plant Microbe Interact doi.org/10.1094/MPMI-06-130181-R) 
The complementation behaves again like a wild type strain. This supports the role $C P C 1$ plays for pathogenocity of the fungus.

To confirm these data DNA quantification was performed for $\mathrm{Vd} \triangle C P C 1$ in comparison to the complementation (Fig. 31). The amount of fungal DNA in the tomato roots, hypocotyls and leaves is reduced for the knockout, but the complementation is on the same level as the wild type (Fig. 31). The DNA concentration is about $40 \%$ lower than in V. dahliae wild type in the hypocotyl and about $50 \%$ for the leaves.

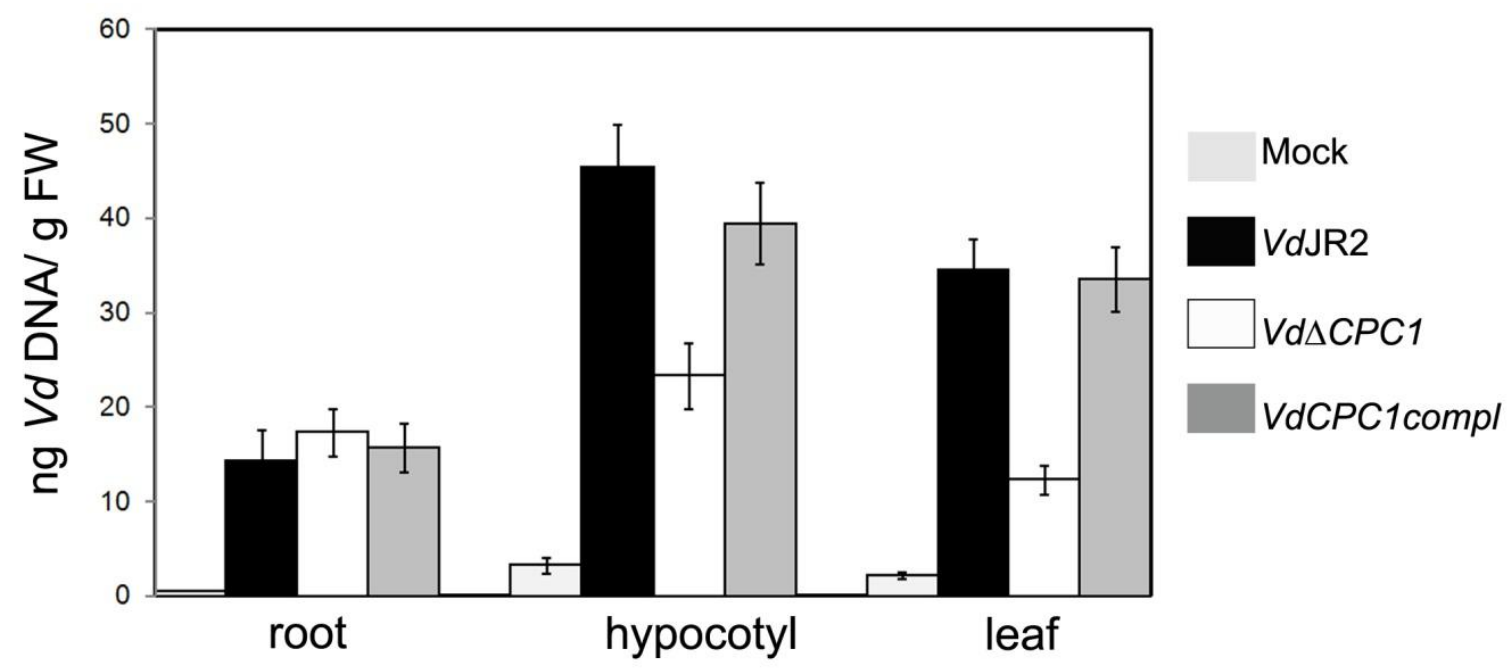

Fig. 31 Infection assay and determination of the $V$. dahliae DNA concentration in infected plant tissues. A. $V$. dahliae DNA concentration in hypocotyls, after 35 dpi. V. dahliae DNA was measured with real-time PCR in root, hypocotyl and leaves of $S$. lycopersicum inoculated with $V d C P C l$ knockout srain ( $V d \triangle C P C l$ ), wild type (VdJR2)and complementation of $C P C 1$ (VdCPClcompl) at 35 dpi. Data represent average \pm standard deviations of five experimental replicates. The mock-inoculated plants as a control did not show presence of any $V$. dahliae DNA. ng Vd DNA/g FW = nanogram $V$. dahliae DNA/gram fresh weight of plant tissue.

Silencing of $C P C l$ in $V$. longisporum leads to a mutant strain that is significantly reduced in pathogenicity. It still can infect the plant but its ability to successful colonization is strongly restricted. Only low amouts of fungal biomass can be found in the upper parts of the host. A knockout of $C P C l$ in $V$. dahliae is also reduced in pathogenicity and shows sensitivity to different plantal carbon sources. 


\subsection{The key regulator of secondary metabolism Lae1: a first glance in Verticillium.}

Secondary metabolism has been described to play a key role in the fungal life cycle (Bayram et al., 2008; Amaike and Keller, 2009; Bayram and Braus, 2012). Regarding phytopathogenic fungi effectors and toxins are involved in many processes providing the organism with defence against the host and counteracting the plantal pathogen response (Howlett, 2006; Mukherjee et al., 2012). In the interactions between soil-borne vascular fungi and plants the mechanisms of pathogenicity implicates the exchange of chemical signals within the vascular tissue. In the interaction between $V$. longisporum and B. napus, both the pathogen and the host plant are supposed to release metabolites into the xylem which affect the other partner. The main regulator for secondary metabolism Lae1/LaeA represents presumably a methyl transferase and has been described in several fungi (Bok and Keller, 2004; Bok et al., 2005; Yu and Keller, 2005; Amaike and Keller, 2009). LaeA has been proposed as an epigenetic regulator which acts on chromatin but exact molecular function is yet elusive. The symptoms caused by the infection indicate that fungal secondary metabolites secreted by the pathogen might play a key role as virulence factors with phytotoxic, elicitor-like or phytohormonal effects, or as suppressors of plant defense. In several studies it has been suggested that Verticillium spp. produces a broad spectrum of phytotoxins and elicitor compounds which induce pathogen-specific symptoms in the absence of the fungus (Shi and Li, 2008; Mukherjee et al., 2012; Zhou et al., 2012).

From previous studies (Ratzinger et al., 2009; Singh et al., 2010; Iven et al., 2012) we could conclude that secondary metabolism is involved in pathogenicity of Verticillium ssp. Therefore we decided to take a closer look on LAEl of $V$. longisporum as well as V. dahliae.

\subsubsection{There are two copies of $L A E 1$ in $V$. longisporum}

$V$. longisporum has been described as hybrid between two Verticillium species (Tran et al., 2013). Therefore we determined the copy number of LAE1 in V. longisporum. To determine the copy number of VILAE1 Southern analysis was performed (Fig. 32). Genomic DNA of $V$. longisporum, $V$. dahliae, and V. albo-atrum were digested with suitable enzymes, and subjected to Southern hybridization. A fully sequenced 520 bp long fragment of VlLAE1, amplified with primers ProbeLae1left and ProbeLae1right was used as the probe. Several independent Southern hybridization analyses showed two signals for $V$. longisporum as compared to V. dahliae, and V. albo-atrum for the genomic DNA digested with SalI.

These results demonstrated that VILAE1-1 has an isogene, VILAE1-2. To determine the extent of similarity of these two isogenes in the $V$. longisporum genome, the genomic DNA of 
$V$. longisporum was digested with SalI and run on a low percentage agarose gel. DNA was extracted from the four gel fragments corresponding to spots where the signals were generated in the Southern hybridization analysis (Fig. 32). VILAE1-1 specific primers were used to amplify the gel extracted DNA and subjected to sequencing. On sequencing of both loci it was found out that with some exchanges the coding and intronic sequences of both the isogenes are identical in $V$. longisporum. We have analyzed and compared the sequences from $V$. longisporum isolates Polymerase chain reaction (PCR) amplification using primers specific for VlLAEl (Table 3) resulted for each primer pair in only one single sequence characteristic for each analysed strain of the three species.

VI $43 \quad$ Vd $\quad$ Va

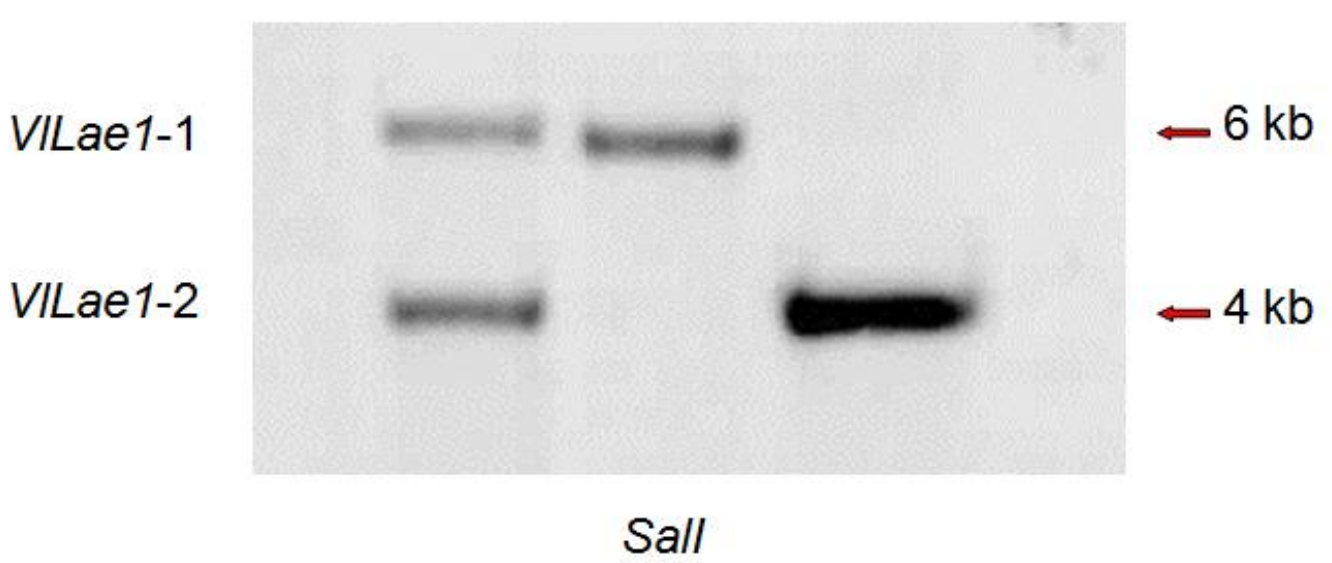

Fig. 32 Determination of the isogene VILAE1-2 of VILAE1-1 and their corresponding sequence. Southern hybridization analysis of V. albo-atrum, V. dahliae and V. longisporum. The genomic DNA was digested with SalI. A 500 bp sequence of VlLAE1-1 was used as a probe.

The sequences for $V$. albo-atrum and $V$. dahliae share $88 \%$ of identity. In total there are more than 60 different nucleotides between the two sequences. Comparing the sequences of the two isogenes of $V$. longisporum to $V$. dahliae or $V$. albo-atrum shows that one of those sequences is similar to $V$. dahliae and the other one is related to V. albo-atrum.VILAE1-1 is 97\% identical to VdLAE1 and they differ in 15 nucleotides. VILAE1-2 is $90 \%$ identical to VdLAE1 and they differ in 45 SNPs (Fig. 33). Compared to VaLAE1 VILAE1-1 is like VdLAE1 $82 \%$ identical to this sequence, whereas VILAE1-2 is at least $89 \%$ identical. There 
are two characteristic sequence patterns which correspond either to $V$. dahliae or to $V$. alboatrum. VILAE1-1 and $V$. dahliae share three patterns that are identical (TGGCT), (GCCGA) and (GCTTGGT). First VlLAE1-2 shares SNPS in the first and the third exon of V. alboatrum (TTACT) and (GATAGGT), but the SNP in the second exon (GCCGA) is similar to $V$. dahliae.
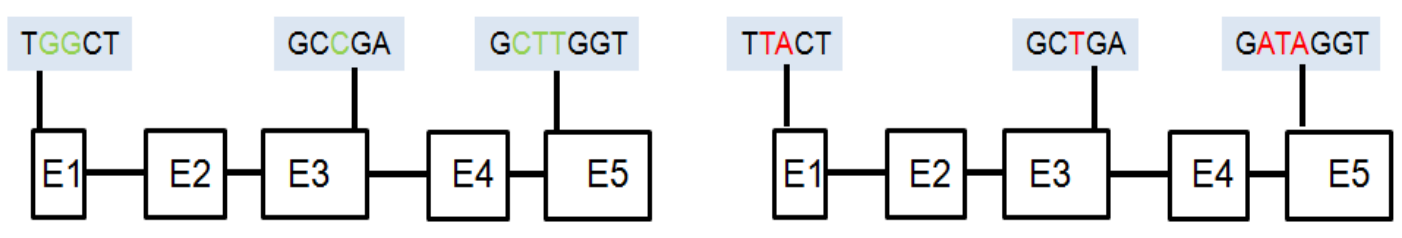

VdLae1

VaLae1
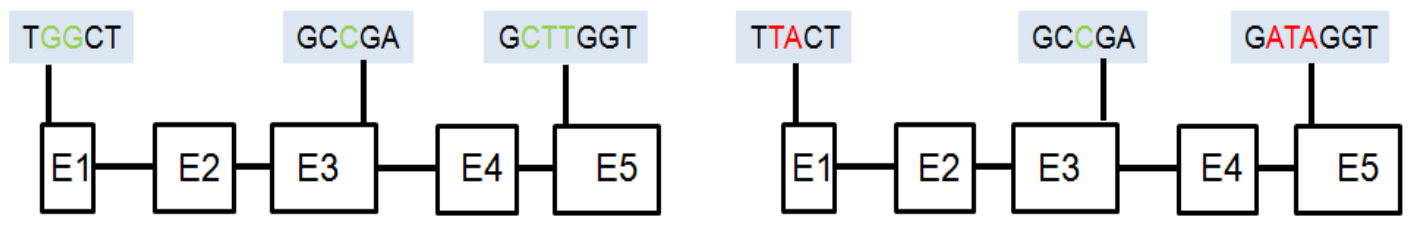

VILae1-1

VILae1-2

Fig. 33 Sequence comparison of VILAE1 sequences. VILAE1-1 and VILAE1-2 with Vdlae1 and Valael. VILAE1-1 and VILAE1-2 isogenes of $V$. longisporum are derivatives from V. dahliae and V. albo-atrum, respectively. Characteristic signatures include two five nucleotide (TGGCT and GCCGA) patterns and a seven nucleotide pattern (GCTTGGT) for VILAE1-1 and the V. dahliae orthologue. Whereas VILAE1-2 corresponds to the $V$. albo-atrum orthologue carries different nucleotide pattern for the first signature (TTACT) the second pattern corresponds to V. dahliae (GCCGA) and not to V. albo-atrum (GCTGA), while the third one is similar to $V$. albo-atrum again (GATAGGT).

The deduced Lae1 protein sequences of $V$. longisporum $V$. dahliae and $V$. albo-atrum were compared with corresponding sequences of A. fumigatus, A. nidulans, P. citrinum or $N$. tetrasperma (Fig. 34). VlLae1-1 and VdLae1 are 99,7\% identical, while VlLae1-2 is 96,9\% identical to both sequences (Fig. 34). VaLae1 is 64,7 \% identical to VlLae1-1 and VdLae1, but $79,2 \%$ identical to VlLae1-2. We can conclude that the one copy of Lae1 in $V$. longisporum refers to $V$. dahliae while the other one is closer to V. albo-atrum, but still closer to the other two copies in $V$. longisporum or $V$. dahliae. 
VILae1-1

V. dahliae Lae

VILae1-2

.albo-atrum Lae1

N. tetrasperma

A. nidulans

A. fumigatus

P. citrinum

VILae1-1

V. dahliae Lae1

ViLae1-2

V.albo-atrum Lae1

N. tetrasperma

A. nidulans

A. fumigatus

. citrinum

VILae1-1

V. dahliae Lae1

ViLae1-2

V.albo-atrum Lae1

$\mathrm{N}$. tetrasperma

A. nidulans

A. fumigatus

P. citrinum

VILae1-1

V. dahliae Lae1

VILae1-2

V.albo-atrum Lae1

N. tetrasperma

A. nidulans

A. fumigatus

P. citrinum

VILae1-1

V. dahliae Lae1

VILae1-2

V.albo-atrum Lae1

N. tetrasperma

A. nidulans

A. fumigatus

$P$. citrinum

VILae1-1

V. dahliae Lae1

VILae1-2

V.albo-atrum Lae1

$\mathrm{N}$. tetrasperma

A. nidulans

A. fumigatus

$P$. citrinum

VILae1-1

V. dahliae Lae1

VILae1-2

V.albo-atrum Lae1

N. tetrasperma

A. nidulans

A. fumigatus

P. citrinum

VILae1-1

V. dahliae Lae1

Vilae1-2

V.albo-atrum Lae1

N. tetrasperma

A. nidulans

A. fumigatus

P. citrinum

VILae1-1

V. dahliae Lae1

VILae1-2

V.albo-atrum Lae1

N. tetrasperma

A. nidulans

A. fumigatus

P. citrinum

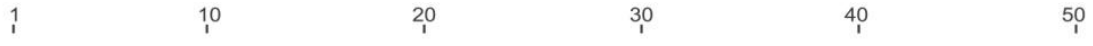

MTS PAHNHYS YHSPTSISDR G-MSYRES PGSFPAPDRTS MELNGQGGQR--PPMNAFPP LNVRGS ISSG FNALGPP TINS PEP PKR YKS FSTPASIDA

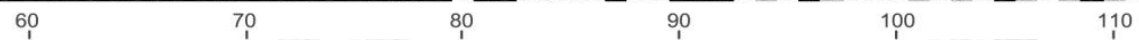

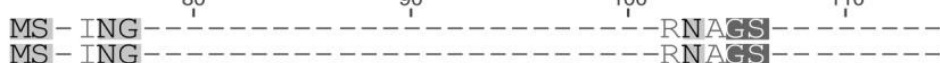
MS - ING---

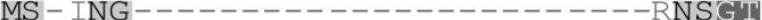
MS - TGYYS PSAAPPDSKCSYTANOS SVTSRNSCI - - -

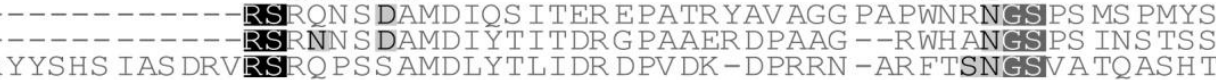

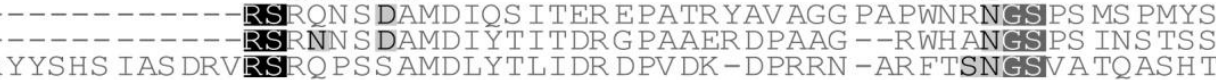
120 140 150 160 QGYRENGRVYGEFR GRYLFPIDEEEKDREDV'H QGYRENGRNYGEFR GR YLEPIDEFEKDREDV IH QGHRENGRVYGEFR GR YLFPIDEEEKDR DDVFH QGHRENGRVYGEFR GR YLFPIDEFEKDR EDVFḦ

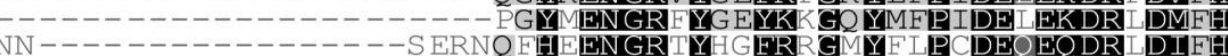
CY SNTSQV SRSS P I IMS DRK I PEKY PNHKENGRMYH YRKG YPI PCDEEEODR LDIFH

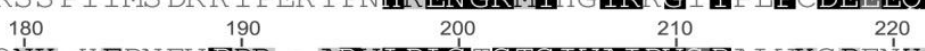

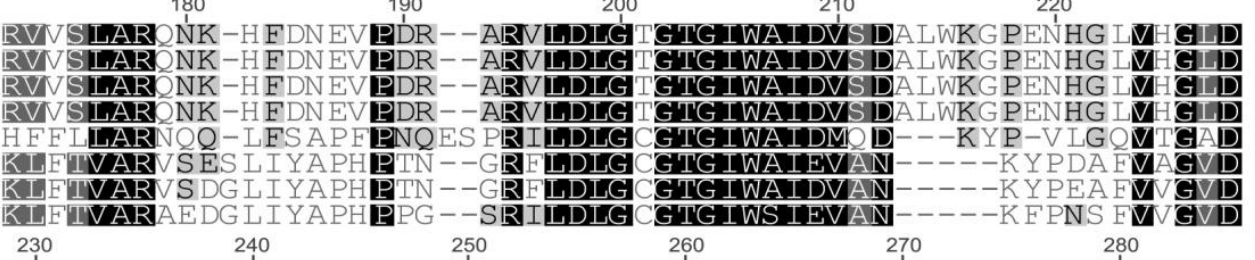
230
260

LAYIOPADVPS T'VS EMO -ADVEEIPWP - IRR END'YDMIHIOLLK'GGIR DWT DI YRIKIFR

LAYIOPADVPS TVS EMO - ADVEEPWP - LR ENDYDMIH IOLLKGG IR DŴT DI YRIK IFR

LAY IOPADVPS TVS FMQ -ADVEEPWP - LR ENDYDIIHIOLLMGG IR DW̄ADLYRKIFR

LAYIOPADVPS TVS EMO -ADVEEPWP - LR ENDYDLIH IOLLMGGIR DWTTDYRK IFR

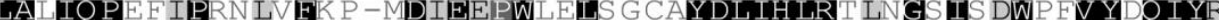

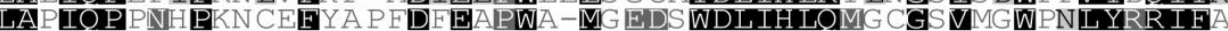

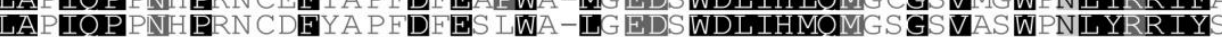

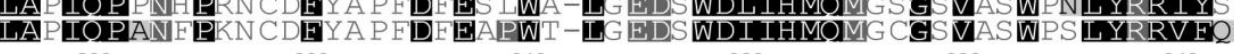
290

HIIKPGG -YTEH VEIDWT FRSIDDNTITS T A TVANSEAVHRIALRTFGTPIDIN-DR IK

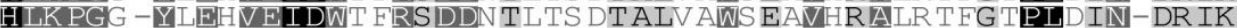
HIKPGG - VT THNETDWT FRSDDNTITS TALVANS EANHRITRTFGTPTDIN-DR IK HLK ISSGG HIKRH CGEV EQVEIDWV PRS DDR S LPPDS F FRWAEALV EIMMRAGR PMR L DS HRVQ HIRPGA - WFEQ VEIDFE PRCDDRS - LDG T ALRHWY DCLKQ IATAETMR PAHISR DT HILPGA - WFEQ VEIDFE PRIDDDR - LEG LA IRQ WYQ LLKQATEETMR PVAHNSRETI HLR PGA - WFEOVEIDFR PRVEDK DGEPGR AMAS WYS TLKH ATEATMR PIAHSSNET I 350 
Lae1 of Aspergillus shares 34,5\% identity with VdLae1 and VlLae1-1, 35,5\% for VlLae1-2 and $31,3 \%$ with VaLae1. A. fumigatus shares $33,1 \%$ identity to Verticillium sequences, while N. tetrasperma is $43,2 \%$ identical, $P$. citrinum is $31 \%$ identical (Fig. 34 ).

\subsubsection{Generation of mutants silenced in $L A E 1$.}

Because there are two copies in V. longisporum mutants silenced in VILAEl were generated. RNA-mediated gene silencing has been established in several fungi for targeted gene silencing instead of a conventional knockout (Nakayashiki et al., 2005). The plasmid includes a hairpin construct for silencing of VlLAE1 and the hygromycin resistance gene as selective marker. A. tumefaciens mediated transformation of $V$. longisporum was applied and thirty independent hygromycin-B-resistant transformants were selected at random for further analysis. The efficiency of VlLAEl silencing was determined by RT-PCR as the silencing of gene expression is the result of posttranscriptional degradation of targeted mRNA. Total RNA was extracted from the VILAE1sm (VlLAE1 silenced mutant) and wild type. RT-PCR results showed the significant knockdown of VlLAE1 transcripts (Fig. 35). The extent of silencing varied in the different transformants between high and moderate levels. The degree of silencing of cross pathway control genes was estimated by RT-PCR by the ratio of the amplification of VILAEl between the respective transformants and the unsilenced wild type. For each transformants three biological replicates were analyzed. High-frequency silencing was observed for $45 \%$ of the transformants exhibiting reduced gene expression. The extent of silencing varied in the different transformants between high and moderate levels. After 5 generations on SXM Medium, the silencing of the VlLAE1 isogenes was still stable (Fig. 35).

A

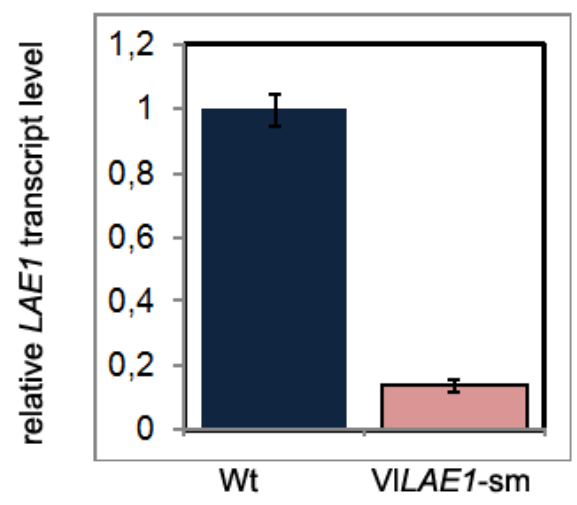

B

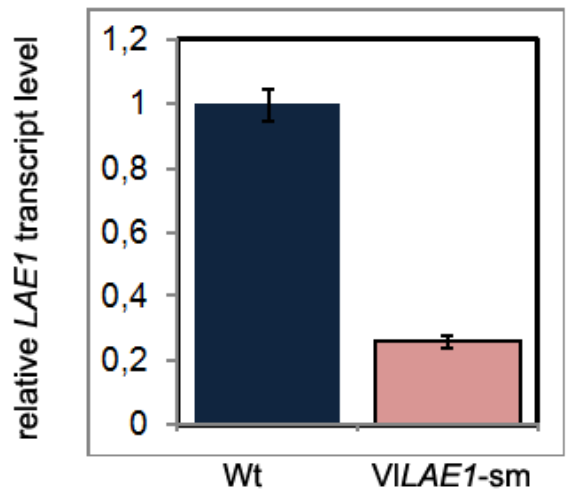

Fig. 35 Expression of lae1 encoding genes of $V$. longisporum. 
Relative expression of VILAE1 measured by quantitative real-time PCR. VILAE1 cDNA was normalized to the histone cDNA. Relative expression of VlLAE1 in $V l 43$ and VILAEI-sm measured by quantitative real-time PCR. VILAEI cDNA was normalized to the histone cDNA. Vl 43: wild type, VILAE1-sm, lael silenced mutants. The error bars represent the standard deviation of four different measurements of the same cDNA. A. Transcript levels of VILAE1 B. Transcript levels of LAE1 after 5 generations on SXM.

As mentioned above for CPC1 silencing strategy suppresses maximally about $80-90 \%$ of gene expression in fungi (Nakayashiki et al., 2005; Singh et al., 2010; Singh et al., 2012). The rest activity of the gene (10-20\%) might be still enough for its function in the fungus. Therefore we decided to generate a knockout of LAEl in $V$. dahliae. To analyse mutants and wildtype strain, a probe binding to the 5 ' region of the gene was used. Genomic DNA isolated from transformants was digested with the enzymes StuI and SalI, for comparison wild type DNA was used. From Southern hybridisation, the 456-bp fragment of LAE1 gene as probe detected one band for LAEl gene in both wild type and mutant but both bands are visible in the ectopic background (Fig. 36). The transformants displayed the expected signals at $1.5 \mathrm{~kb}$ (StuI and SalI) (Fig. 36). The Southern band from the deletion mutants was $1 \mathrm{~kb}$ smaller than the band from the wild type strain explaining the successful exchange between the deletion cassette and locus of $L A E 1$ gene. For final validation Southern hybridisation was made with the same probes and enzymes as for the two single knock-outs, and isolates resulting in the correct bands were used for further investigation (Fig. 36). 


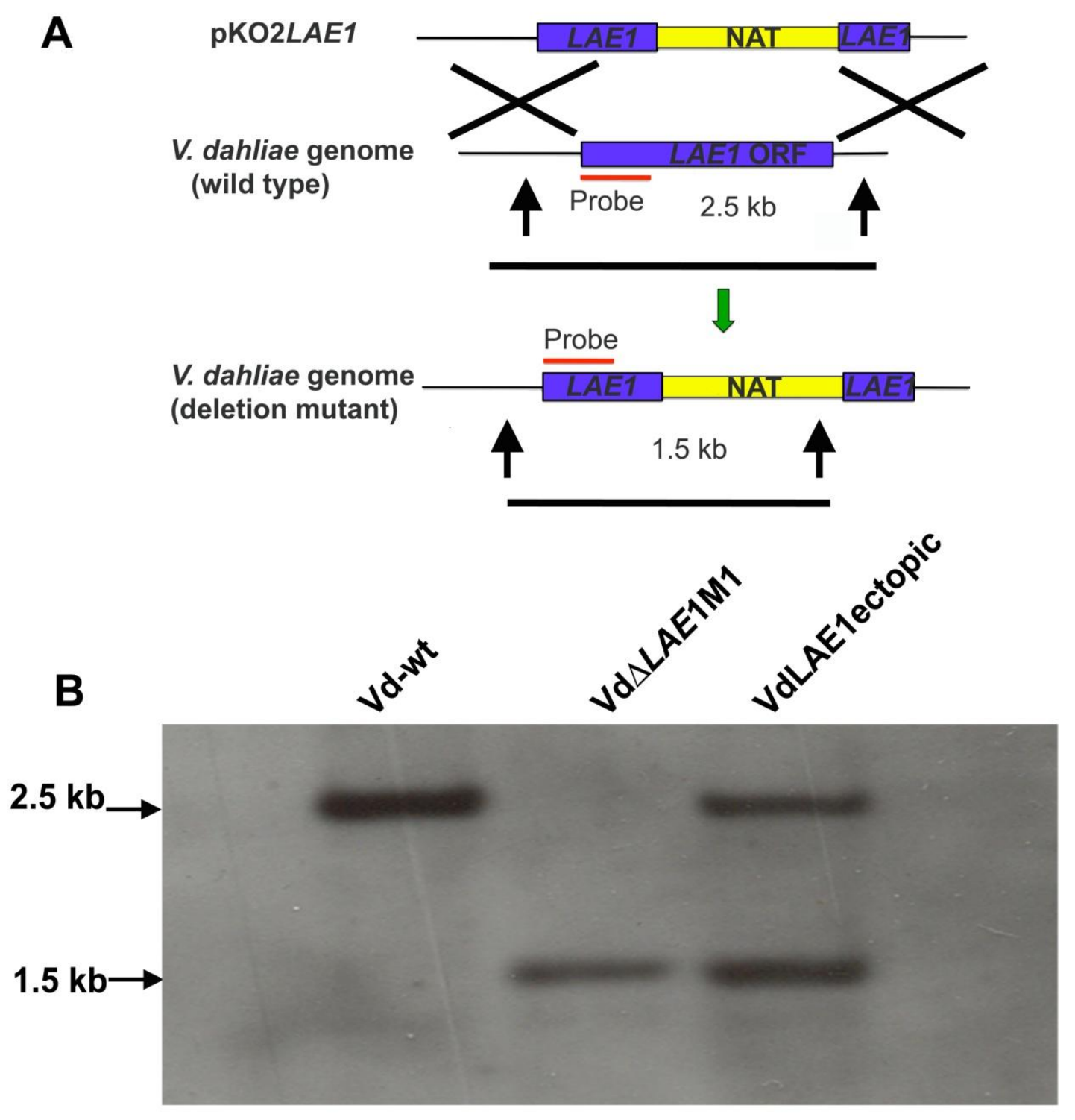

Fig. 36 Strategies for deletion of $L A E 1$ gene in $V$. dahliae and confirmation of the corresponding deletion mutants. A. Model for exchange between the deletion construct and wild type locus of LAE1 gene in $V$. dahliae. B. Confirmation of the LAEl deletion mutants by Southern hybridization using StuI and SalI for genome digestion. The same band $(1,5 \mathrm{~kb})$ in the mutants is $1 \mathrm{~kb}$ smaller than the one in the wild type VdJR2.

\subsubsection{Silencing of $L A E 1$ reduces pathogenicity of $V$. longisporum.}

$V$. longisporum mutants silenced in expression of $L A E 1$ were tested in a plant infection assay to verify the degree of disease. The same symptoms like for silencing of $C P C l$ could be observed for VILAElsm.

In case of $L A E 1$ silencing treatment and observation of the plants was carried out like discribed above. In the initial phase of the disease the infected and non infected do not show severe differences, but with development at 21 and 28 dpi distinctions between the wt infected plant and the plants infected with VILAE1sm become obvious. At 35 dpi, the disease 
score of the VILAElsm-infected plants was lower than the plants infected with wildtype fungus, but still significant in comparison to mock-inoculated control plants (Fig. 37). This reflects a slow down of the disease of the plant when infected by the mutant fungi corroborating a reduced impact on senescence. The infected plants did not show any disease symptoms until $14 \mathrm{dpi}$. The disease score of the VlLAElsm-infected plants was higher (4.2) than the mock-inoculated plants $(3,0)$ but lower than the plants infected with wild type $(6.0$; Fig. 37). V. longisporum DNA in plant tissue infected with VlLAElsm correlate with reduced virulence. 
A

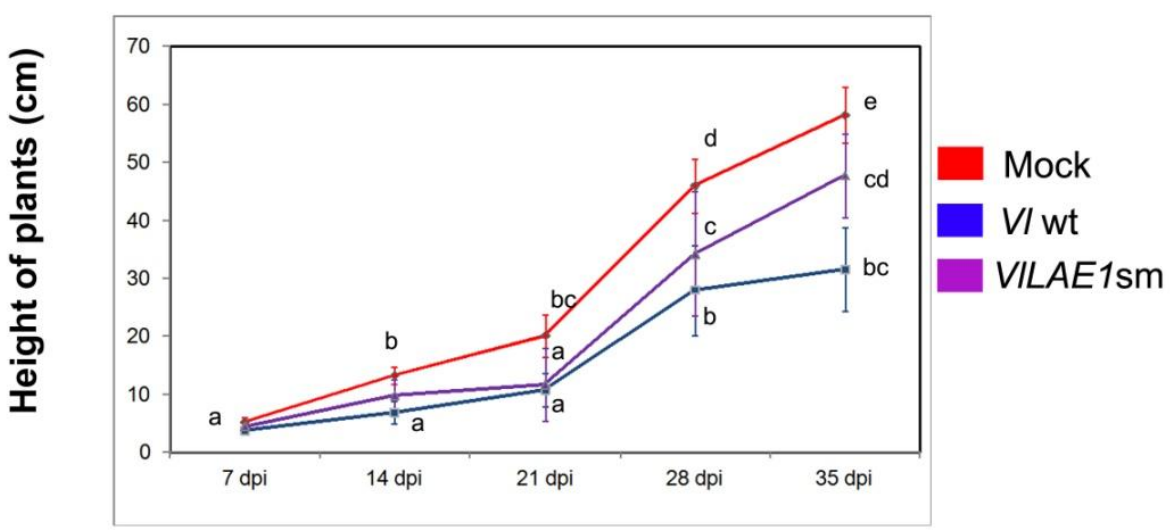

B

Days post infection

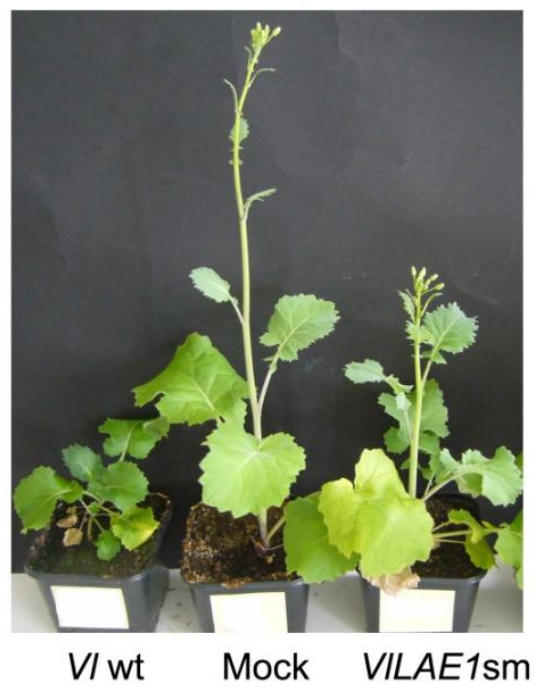

C

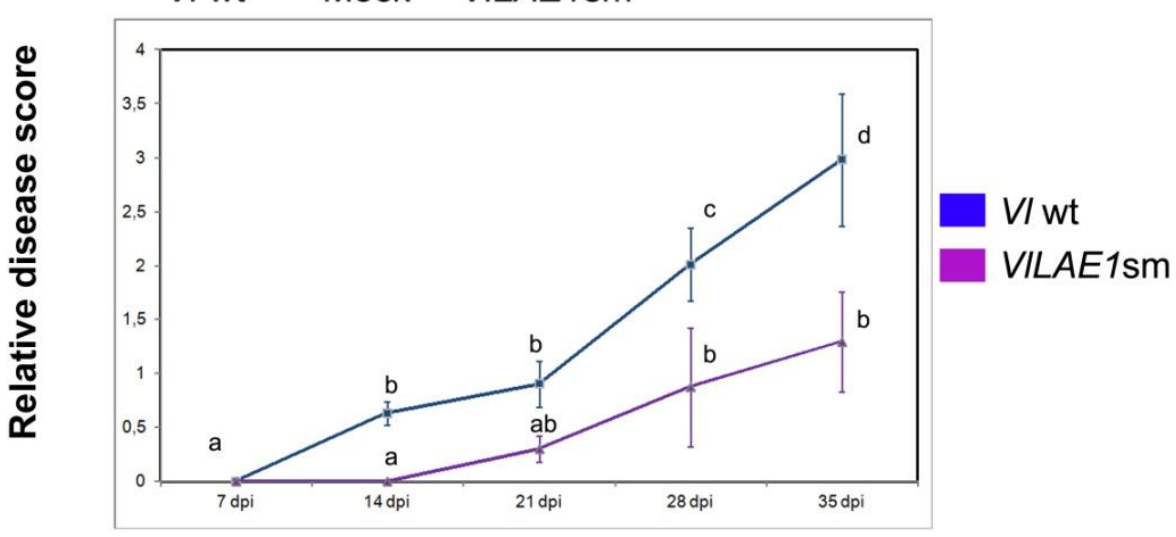

Days post infection

Fig. 37 Assessment of pathogenicity of the VILAE11 silenced mutant. A. Assessment of stunting of rapeseed due to $V$. longisporum infection. The height of 30 replicates each of rapeseed plants infected with wild type (wt) and VlLAE1 silenced mutant (VlLAE1 sm) was measured at 7, 14, 21, 28, and 35 dpi. For comparison, the height of rapeseed plants mock-inoculated (mock) with tap water was also measured. The plants are heavily infected at 28 and 35 dpi. Data represent average \pm standard deviations of 30 experimental replicates. B. Rapeseed infection assay. Representative B. napus plants shown at 35 dpi. C. Assessment of disease development by scoring for disease symptoms according to Eynck et al. (2007). Plants were scored for disease symptoms at 7, 14, 21, 28, and $35 \mathrm{dpi}$. Data represent average \pm standard deviations of 30 experimental replicates. $V l$ 43, wild type; $V I L A E 1 \mathrm{sm}, V I L A E 1$ silenced mutant. Different letters indicate significant differences at $\mathrm{P}<0.05$. 


\subsubsection{A deletion of $L A E 1$ doesn't affect the pathogenicity of $V$. dahliae.}

To investigate whether a deletion of $L A E 1$ also affects pathogenicity of $V$. dahliae to its host we performed the infection assays on tomato plants of the VdLAE1 deletion mutant $(\mathrm{Vd} \triangle L A E 1)$ and the $V$. dahliae wildtype by root-dipping method. The results showed that the mutant is infecting the plants with reduced efficiency whereas the wild type is successfully colonizing its host (Fig. 38). The wild type $V$. dahliae caused the typical symptoms on tomato including stunting and chlorosis (Fig. 38), as well as the LAE1 deletion mutant.

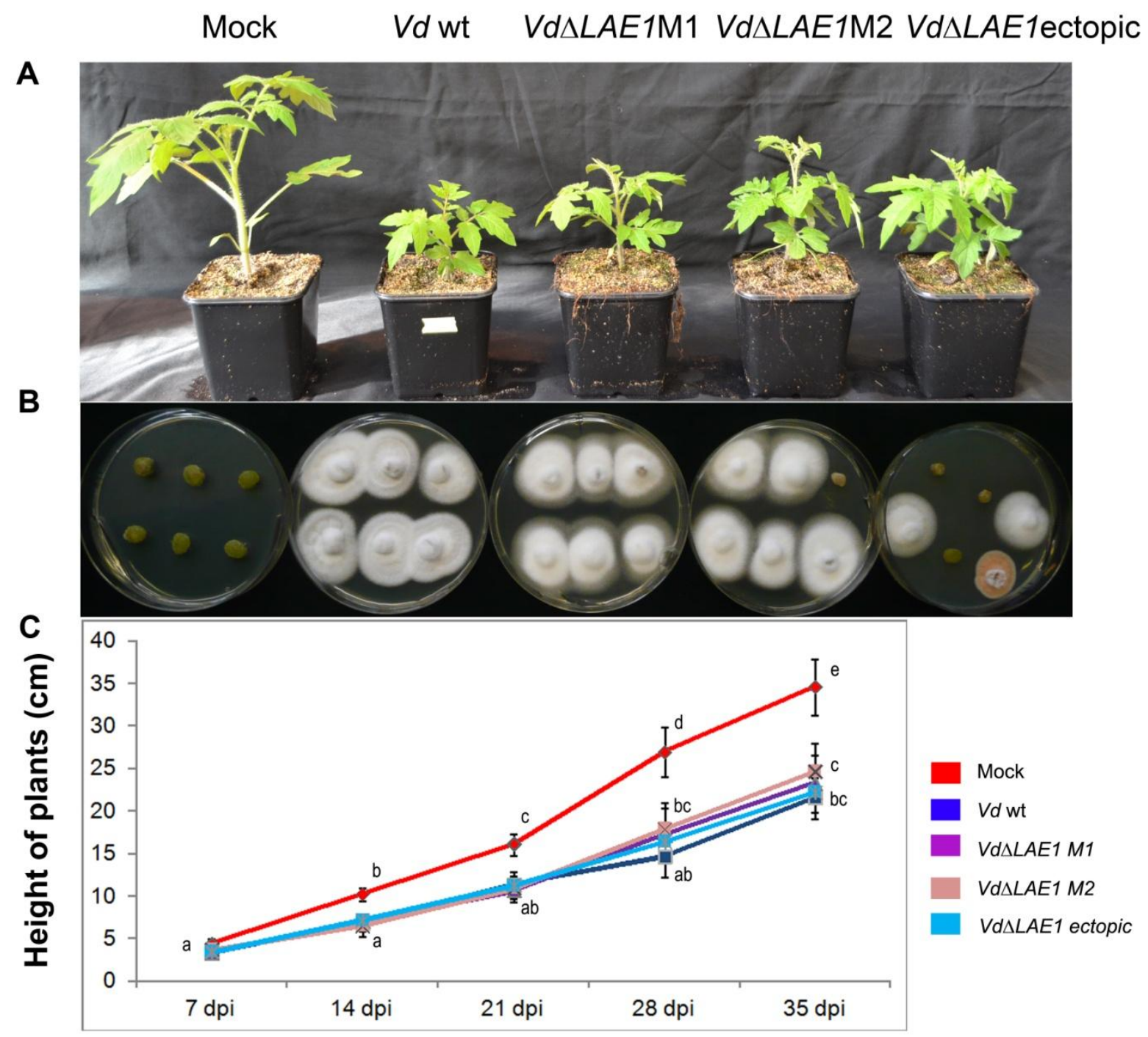

Days post infection

Fig. 38 Assessment of pathogenicity of the VdLAE1 deletion mutant an complementation. A. Tomato infection assay. Representative S. lycopersicum plants shown at $21 \mathrm{dpi}$. B. Mutant regrowth test. Image of 
tomato stem pieces infected with $V$. dahliae or $V d L A E 1$ knockout mutant ( $\mathrm{Vd} \triangle L A E 1)$ colonies growing PDA plates. As control stem pieces of Mock served as control. C. Assessment of stunting of S. lycopersicum due to $V$. dahliae infection. The height of 20 replicates each of tomato plants. Different letters indicate significant differences at $\mathrm{P}<0.05$.

Compared to silencing of LAE1 in $V$. longisporum deletion of the corresponding gene in $V$. dahliae did not show an effect on pathogenicity. The plant height of wild type infected plants was not different from the mutant infected plants. In average they are $22 \mathrm{~cm}(\mathrm{Vd}-\mathrm{wt})$ until $24 \mathrm{~cm}(\mathrm{Vd} \triangle L A E 1 \mathrm{M} 1)$. The non infected plants are around 6-8 $\mathrm{cm}$ heigher than the infected ones.

We analyzed whether VILAElsm mutants were still able to colonize B. napus and cause disease, and whether there is also reduced growth of the mutant in the plant or whether the plant is able to inhibit fungal growth. Therefore, the total biomass of $V$. longisporum in B. napus infected with VlLAElsm versus wild type was analyzed by quantification of the $V$. longisporum DNA in the plant tissue by real-time PCR. The roots hypocotyls, stems and leaves were harvested from the rapeseed plants infected with wild type or VILAE1sm at various time points (Fig. 39).

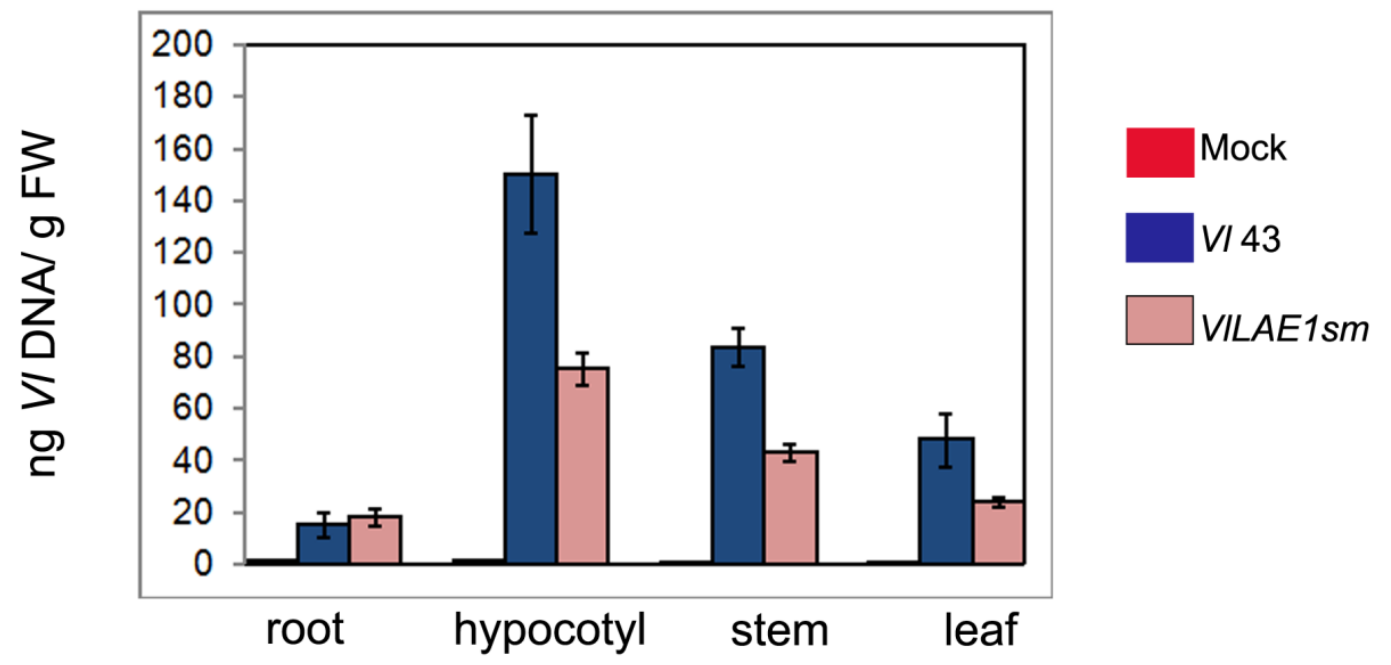

Fig. 39 Infection assay and determination of the $V$. longisporum DNA concentration in infected plant tissues. Different letters indicate significant differences at $P<0.05$. A. V. longisporum DNA concentration in root, hypocotyls, stem and leaves after 35 dpi. V. longisporum DNA was measured with real-time PCR in stem, hypocotyls, root and leaves of B. napus inoculated with VILAE1 silenced mutant (VILAE1sm) and wild type (wt) at $35 \mathrm{dpi}$. Data represent average \pm standard deviations of five experimental replicates. The mock-inoculated plants as a control did not show presence of any $V$. longisporum DNA. ng Vl DNA/g FW = nanogram $V$. longisporum DNA/gram fresh weight of plant tissue.

The DNA content was investigated at 35 dpi where there were no differences within the respective sets of experiments $\left(\mathrm{P}_{(\text {time })}>0.5\right)$. Low concentrations of fungal DNA were found in roots, they did not vary between $V l 43, V I l A E 1$ sm infected plants, but show a difference 
towards the mock inoculated plants (Fig. 39). In the hypocotyl the concentration of fungal DNA reaches $150 \mathrm{ng} / \mathrm{g} \mathrm{FW}$ for the wt and around $80 \mathrm{ng} / \mathrm{g} \mathrm{FW}$ for the VILAElsm. For the stem amount of DNA reaches $80 \mathrm{ng} / \mathrm{g}$ FW for wt infected plant and $50 \mathrm{ng} / \mathrm{g} \mathrm{FW}$ for the mutant, whereas for the leaves it is $50 \mathrm{ng}$ for wt and 30 for the mutant. The value for mock is only at basal levels. These data suggest that the mutant strain is able to perform the initial colonization of the plant and also reaches the upper parts of the plant but the distribution in these parts seems to be less effective (Fig. 39).

Silencing and deletion of $L A E 1$ lead to contradictory results. While the silenced mutants of the hybrid $V$. longisporum seems to affect the pathogenicity of the fungus on oilseed rape, deletion of this gene in haploid $V$. dahliae doesn't influence the pathogenicity in tomatoe. Possible Explanations to this problem will be discussed in the Discussion.

\subsection{Triacylglycerol lipase (Tagl) a secreted lipase in V. longisporum.}

Successful infection and colonization of the host requires proteins or enzymes that are capable of acquiring nutrition for the fungal pathogen, or provide opportunities of breaking the plant cell wall. Secreted proteins and secondary metabolites are the key players for these reactions (Mattinen et al., 2007; Ratzinger et al., 2009; Fu and Wang, 2011; Kulye et al., 2012). Furthermore they are directly involved in the pathogenicity of the pathogen. These proteins or enzymes might be useful to adapt to the plants pathogen reaction or either might be important to counteract the pathogen response of the host plant. Among these enzymes, lipases and cutinases, with their potential roles in the hydrolyzation of the plant cuticle and surface waxes, may contribute to pathogen infection at an early stage, when the fungus first comes into contact with its host (Mattinen et al., 2007; Floerl et al., 2012; Iven et al., 2012; Kulye et al., 2012; Singh et al., 2012).

In previous studies (Timpner, 2008) secreted proteins of $V$. longisporum have been analyzed. Therefore a culture of $V$. longisporum was treated with xylem sap and grown under certain conditions (Fig. 40). The secretome of this culture was harvested and further analyzed. One of the identified proteins was a triacylglycerol lipase (TAGL) which was further investigated. Due to the treatment with xylem sap the lipase was upregulated (Fig. 40). The expression level after treatment increased 2,5 fold compared to non inducing conditions. 
SXM secretome of V.I.

- Xylem sap

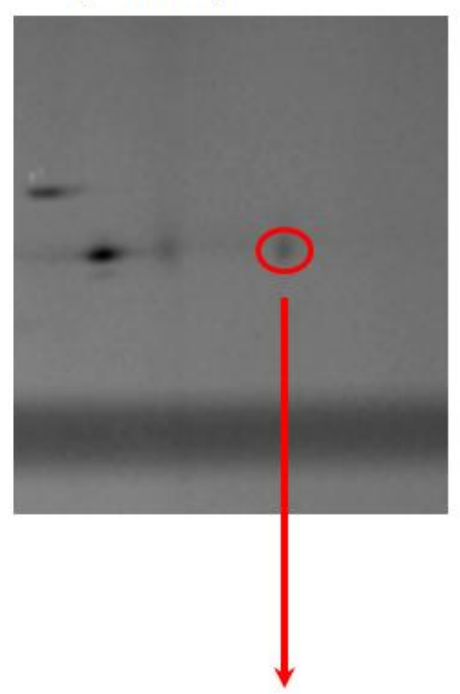

Triacylglycerol lipase
SXM secretome of V.I.

+ Xylem sap
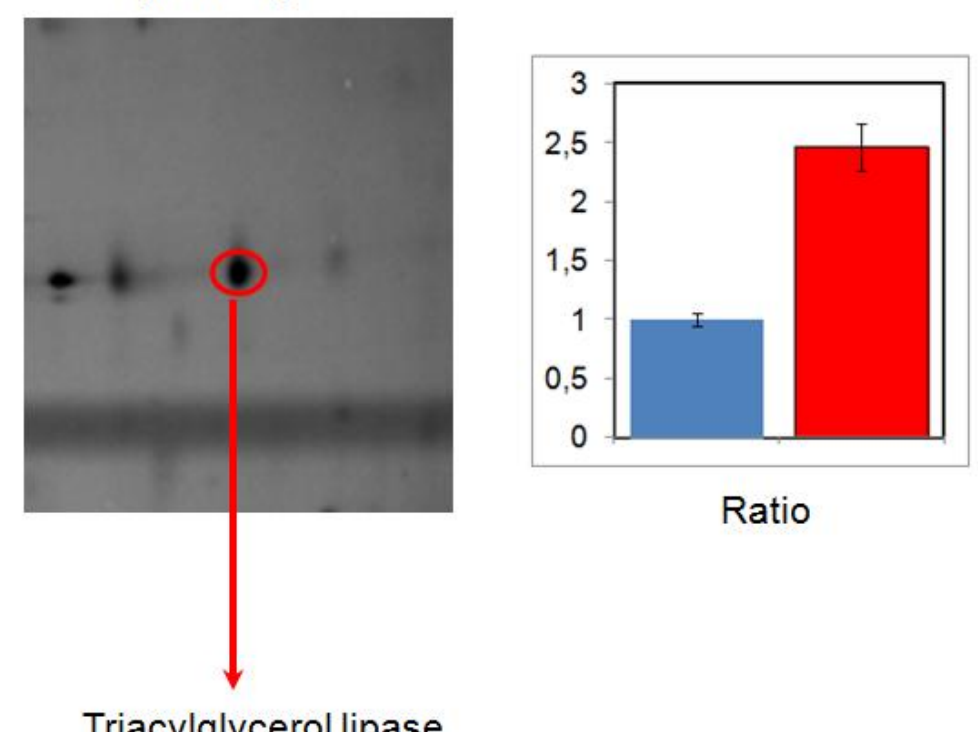

Ratio

Fig. 40 Expression of triacylglycerol lipase after treatment with xylem sap. Close-up views of the regions of the 2-DE gels that show significant upregulation in protein expression (untreated (-Xylem Sap) versus treated (+Xylem Sap) with xylem sap from rapeseed). The red circles indicate triacylglycerol lipase. The expression histograms show the differential protein expression of $V$. longisporum due to treatment with xylem sap from rapeseed (right).

\subsubsection{V. longisporum has two copies of triacylglycerol lipase}

As mentioned for CPC1 and LAE1 V. longisporum has been described as hybrid between two Verticillium species (Inderbitzin et al. 2011). Therefore we determined the copy number of TAGL in V. longisporum. To determine the copy number of VlTAGL Southern analysis was performed (Fig. 41). So genomic DNA of V. longisporum, V. dahliae, and V. albo-atrum were digested with suitable enzymes, and subjected to Southern hybridization. A fully sequenced 520 bp long fragment of VlTAGL, amplified with primers ProbeLipleft and ProbeLipright was used as the probe. Several independent Southern hybridization analyses showed two signals for $V$. longisporum as compared to $V$. dahliae, and V. albo-atrum for the genomic DNA digested with $B g l \mathrm{I}$

These results demonstrated that VITAGL-1 has an isogene, VITAGL-2. To determine the extent of similarity of these two isogenes in the $V$. longisporum genome, the genomic DNA of $V$. longisporum was digested with SalI and run on a low percentage agarose gel. DNA was extracted from the four gel fragments corresponding to spots where the signals were generated in the Southern hybridization analysis (Fig. 41). VITAGL-1 specific primers were used to amplify the gel extracted DNA and subjected to sequencing. On sequencing of both loci it was found out that with some exchanges the coding and intronic sequences of both the 
isogenes are identical in $V$. longisporum. We have analyzed and compared the sequences from $V$. longisporum isolates Polymerase chain reaction (PCR) amplification using primers specific for VITAGL (Table 3) resulted for each primer pair in only one single sequence characteristic for each analysed strain of the three species.

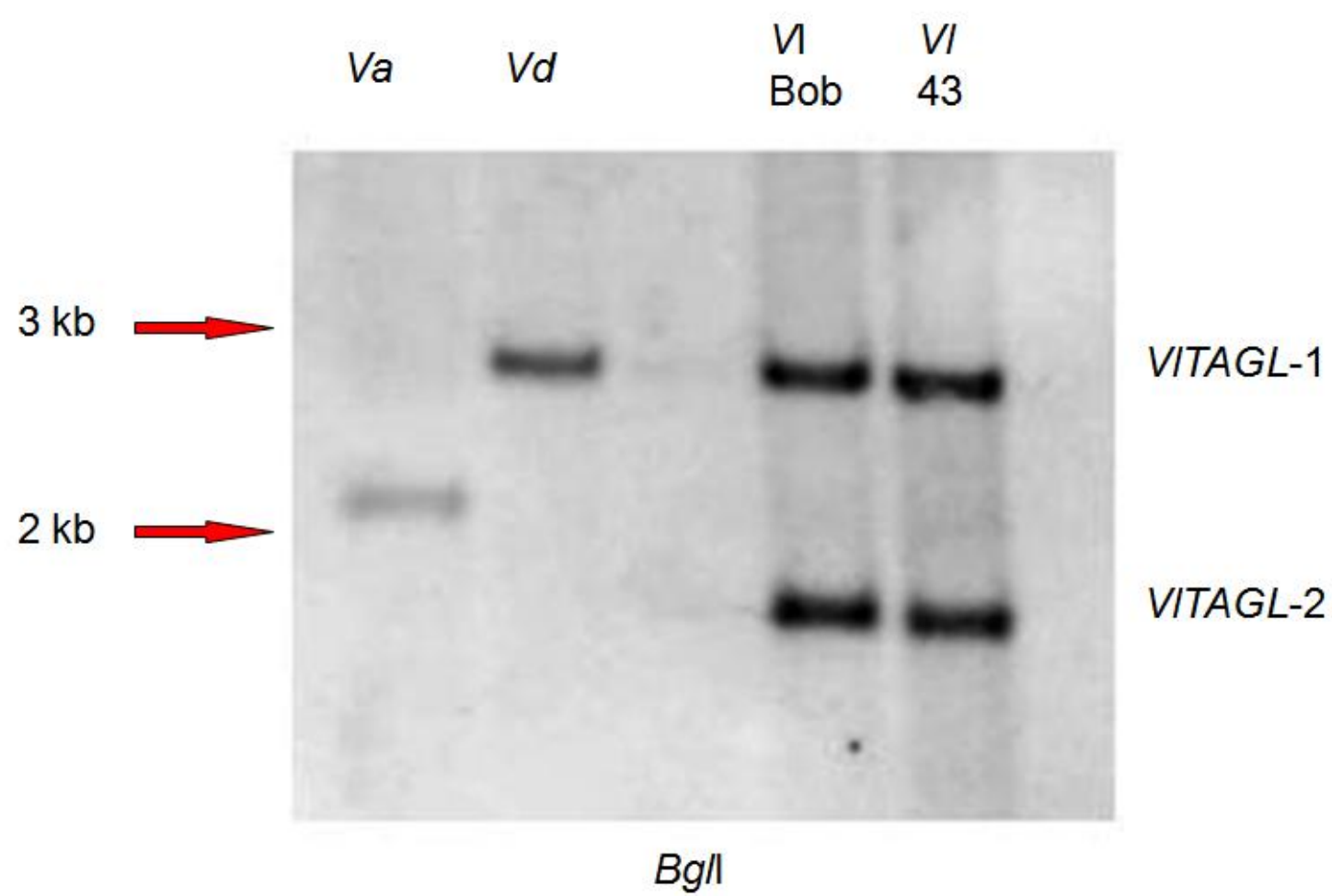

Fig. 41 Determination of the isogene VITAGL-1 and VITAGL-2 and their corresponding sequence. Southern hybridization analysis of V. albo-atrum, V. dahliae and V. longisporum. The genomic DNA was digested with $B g l I$. A 500 bp sequence of VlTAGL- 1 was used as a probe.

The sequences for $V$. albo-atrum and $V$. dahliae share $88 \%$ of similarity. In total there are more than 60 different SNPs between the two sequences. Comparing the sequences of the two isogenes of $V$. longisporum to $V$. dahliae or $V$. albo-atrum shows that one of those sequences is close to $V$. dahliae and the other one is close to V. albo-atrum.VITAGL-1 is $97 \%$ identical to VdTAGL and they differ in 15 nucleotides. VlTAGL-2 is 93\% identical to VdTAGL and they differ in 36 nucleotides (Fig. 42). Compared to VaTAGL VlTAGL-1 is like VdTAGL $88 \%$ identical to this sequence, whereas VITAGL-2 is at least $92 \%$ identical. There are two characteristic sequence patterns which correspond either to $V$. dahliae or to V. alboatrum. VITAGL-1 and $V$. dahliae share two patterns that are identical (GACGGA) and (GCGGGAC). The pattern of VlTAGL-2 correspond in both patterns to V. albo-atrum (GAC-TAA) and (GCGAGCC). 


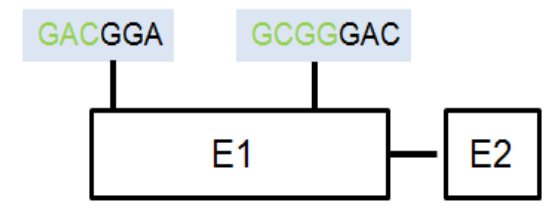

VdTagl

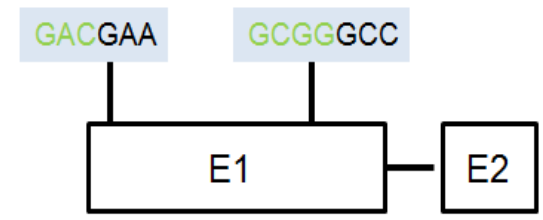

VITagl-1

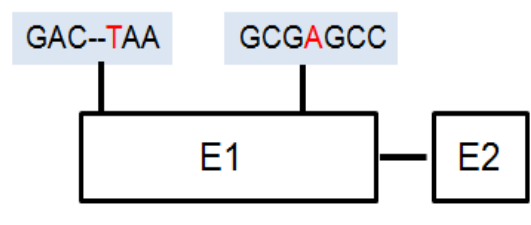

VaTagl

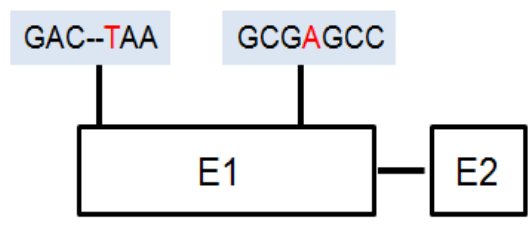

VITagl-2

Fig. 42 Sequence comparison of VITAGL sequences. VITAGL-1 and VITAGL-2 with VdtTAGLand VaTAGL. VITAGL- 1 and VITAGL-2 isogenes of $V$. longisporum are derivatives from $V$. dahliae and $V$. albo-atrum, respectively. Characteristic signatures include a six nucleotide (GACGAA) pattern and a nine nucleotide pattern (GCGGGCC) in the first exon (E1) for VlTAGL-1 and the $V$. dahliae orthologue. Whereas VlTAGL-2 corresponds to the $V$. albo-atrum orthologue carries different nucleotide pattern for the first signature (GAC..TAA), as well as the second one (GCGAGCC).

\subsubsection{Silencing of triacylglycerol lipase in $V$. longisporum}

Like discribed for silencing of CPC1 mutants silenced in VITAGL were generated might be expressed. RNA-mediated gene silencing has been established in several fungi for targeted gene silencing instead of a conventional knockout (Nakayashiki, 2005). The plasmid (for details on plasmid construction see Materials and Methods) includes a hairpin construct for silencing of VITAGL and the hygromycin resistance gene as selective marker. A. tumefaciens mediated transformation of $V$. longisporum was applied and thirty independent hygromycinB-resistant transformants were selected at random for further analysis. The efficiency of VlTAGL silencing was determined by RT-PCR as the silencing of gene expression is the result of posttranscriptional degradation of targeted mRNA. Total RNA was extracted from the VlTAGLsm (VlTAGL silenced mutant) and wild type. RT-PCR results showed the significant knockdown of VITAGL transcripts (Fig. 43). The extent of silencing varied in the different transformants between high and moderate levels. The degree of silencing of cross pathway control genes was estimated by RT-PCR by the ratio of the amplification of VITAGL between the respective transformants and the unsilenced wild type. For each transformants 
three biological replicates were analyzed. High-frequency silencing was observed for $54 \%$ of the transformants exhibiting reduced gene expression. The extent of silencing varied in the different transformants between high and moderate levels. After 5 generations on SXM Medium, the silencing of the VlTAGL isogenes was still stable (Fig. 43).

A

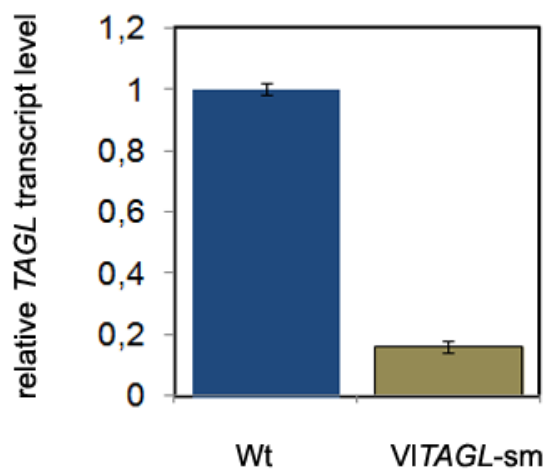

$\mathrm{B}$

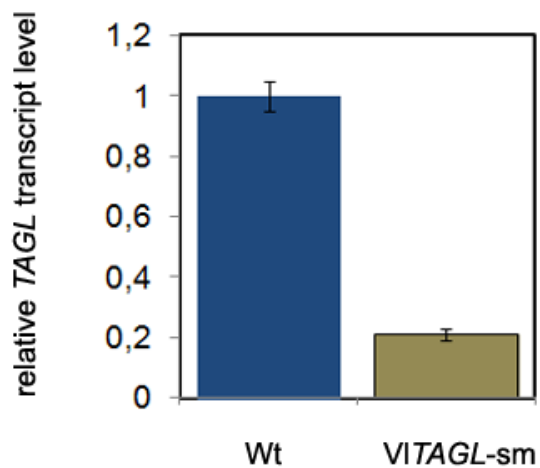

Fig. 43 Expression of TAGL encoding genes of $V$. longisporum. Relative expression of VITAGL measured by quantitative real-time PCR. VITAGL cDNA was normalized to the histone cDNA. Relative expression of VITAGL in Vl 43 and VITAGL-sm measured by quantitative real-time PCR. VITAGL cDNA was normalized to the histone cDNA. Vl 43: wild type, VlTAGL-sm, tagl silenced mutants. The error bars represent the standard deviation of four different measurements of the same cDNA. A. Transcript levels of VITAGL B. Transcript levels of $T A G L$ after 5 generations on SXM.

\subsubsection{Silencing of triacylglycerol lipase in $V$. longisporum shows a reduced pathogenicity of the fungus}

Disease symptoms are categorized be two different sets that can be observed during the infection of oilseed rape by $V$. longisporum. Plant of B. napus show stunting and signs of early senescence. Both symptoms are normally visible after 21 days post infection (dpi) and are more distinctive at later time points. One-week-old B. napus seedlings were infected by root dip inoculation to compare the severity of the disease; height and the signs of early senescence (disease scores) of the plants were measured weekly post inoculation until $35 \mathrm{dpi}$. VlTAGLsm showed the same symptoms like for silencing of $C P C 1$, but in comparison the effects are more pronounced than for the other mutants. In case of TAGL silencing treatment and observation of the plants was carried out like discribed above. In the initial phase of the disease the infected and non infected do not show severe differences, but with development at 21 and 28 dpi distinctions between the wt infected plant and the plants infected with Vllaelsm become obvious. At $35 \mathrm{dpi}$, the disease score of the VlTAGLsm-infected plants was lower than the plants infected with wildtype fungus, but still significant in comparison to mock-inoculated control plants (Fig. 44). This reflects a slow down of the disease of the plant 
when infected by the mutant fungi corroborating a reduced impact on senescence. The infected plants did not show any disease symptoms until 14 dpi. The disease score of the VlTAGLsm-infected plants was higher (4.5) than the mock-inoculated plants $(3,0)$ but significantly lower than the plants infected with wild type (6.0; Fig. 44). V. longisporum DNA in plant tissue infected with VlTAGLsm correlate with reduced virulence. 
A

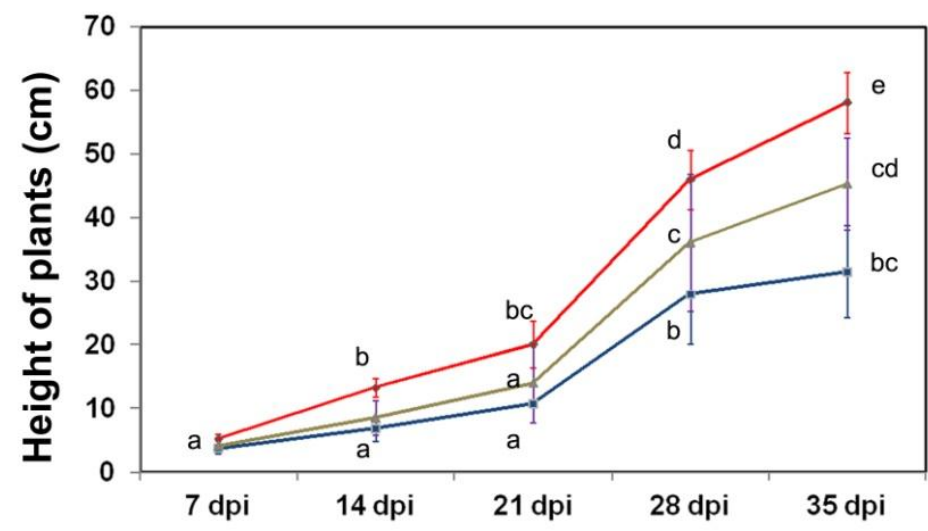

Mock

VI wt

VITAGLsm

B

\section{Days post infection}

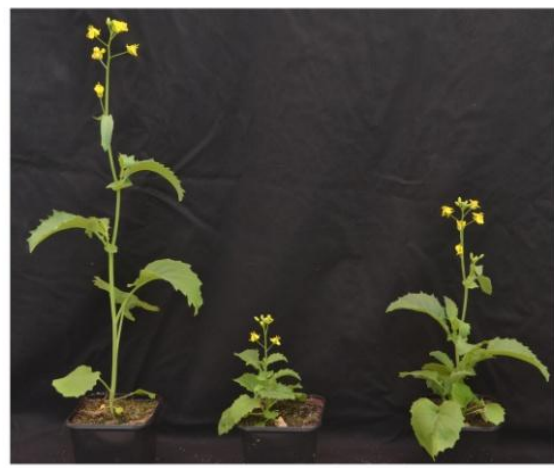

Mock VI wt VITAGLsm

C

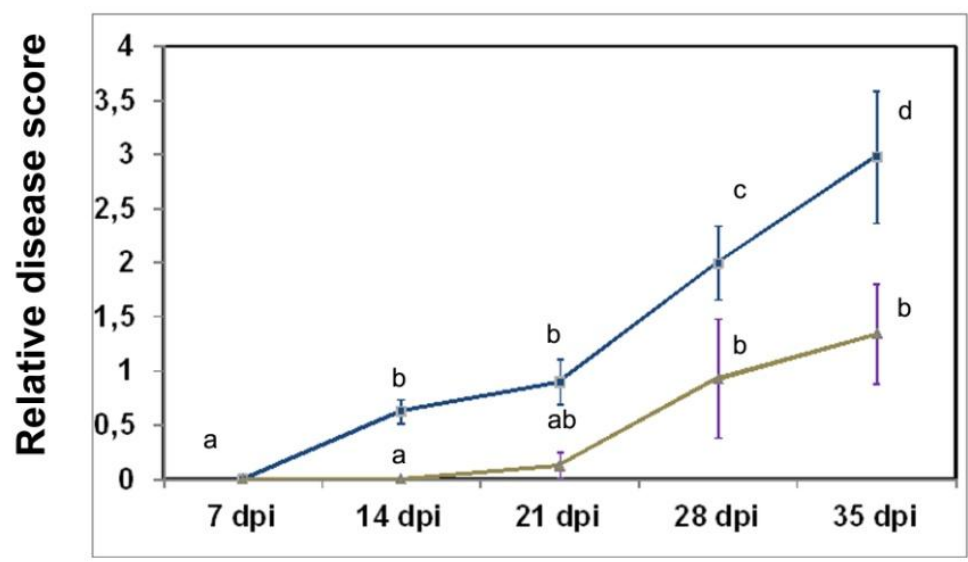

VI wt

VILAE1sm

Days post infection

Fig. 44 Assessment of pathogenicity of the VITAGL silenced mutant. A. Assessment of stunting of rapeseed due to $V$. longisporum infection. The height of 30 replicates each of rapeseed plants infected with wild type (wt) and VITAGL silenced mutant (VITAGLsm) was measured at 7, 14, 21, 28, and 35 dpi. For comparison, the height of rapeseed plants mock-inoculated (mock) with tap water was also measured. The plants are heavily infected at 28 and 35 dpi. Data represent average \pm standard deviations of 30 experimental replicates. B. Rapeseed infection assay. Representative B. napus plants shown at 35 dpi. C. Assessment of disease development by scoring for disease symptoms according to Eynck et al. (2007). Plants were scored for disease symptoms at 7, 14, 21, 28, 
and 35 dpi. Data represent average \pm standard deviations of 30 experimental replicates. $V l 43$, wild type; VITAGLsm, VlTAGL1 silenced mutant. The value for mock was detracted from the values for wild type and mutant. Different letters indicate significant differences at $\mathrm{P}<0.05$ 


\section{Chapter 4. Discussion}

It was the aim of this work to investigate the role of regulatory genes involved in amino acid metabolism like cross pathway control $(\mathrm{Cpc})$ or in secondary metabolism like LAE1. Previous studies (Singh et al., 2010) gave a first hint on the role of $C P C 1$ in V. longisporum. It was demonstrated that the regulatory gene of the cross pathway control $C P C l$ was upregulated when the fungus colonizes the xylem vessels of $B$. napus.

In this work it was shown that (i) the genes for the cross pathway control regulator $C P C 1$ are required for growth in the presence of amino acid analogues and for full virulence of the fungus in planta. Furthermore it was shown (ii) that the LaeA/Lae1 global regulator of secondary metabolism has not a major impact on virulence but (iii) that the secreted triacylglycerol lipase Tagl is required for full pathogenicity on plants.

\subsection{Amino acids metabolism and its role for pathogenicity.}

The cross pathway control has been the subject of examination in several filamentous fungi, including A. nidulans (Piotrowska et al., 1980; Hoffmann et al., 2001), A. niger (Wanke et al., 1997), A. fumigatus (Krappmann et al., 2004; Sasse et al., 2008), C. albicans (Tripathi et al., 2002), $M$ grisea (Shen and Ebbole, 1997), and N. crassa (Carsiotis et al., 1974; Paluh et $a l ., 1988)$. The cross pathway control system of filamentous fungi is an adaptive system that enables the organism to react on different kinds of environmental stress. The function of this regulatory system has been analysed widely and for several organism, including also pathogenic fungi (Carsiotis et al., 1974; Hinnebusch, 1984; Davis, 2000; Piotrowska, 1980; Sachs, 1996; Hoffmann et al., 2001; Krappmann et al., 2004). For example in A. fumigatus this system is required for full virulence (Krappmann et al., 2004). For other plant pathogenic fungi it was anticipated, that a knock down or knock out of $C P C 1$ might reduce pathogenicity (Shen and Ebbole, 1997), but was so far not proven. For pathogenic fungi like A. fumigatus, mutants impaired in cross pathway control system had strongly reduced pathogenicity and CpcA seemed to be involved in establishing pulmonary aspergillosis effectively (Krappmann et al., 2004; Sasse et al., 2008; Abad et al., 2010). The cross-pathway control is a complex global regulatory network, which regulates the synthesis of amino acids in numerous fungi. In the yeast $S$. cerevisiae, the same system had been named general control (Hinnebusch, 1984; Natarajan et al., 2001). Amino acid starvation conditions activate this system resulting in derepression of the cross pathway control gene GCN4 in yeast or $c p c A / c p c-1$ in filamentous fungi, respectively. In yeast the amount of Gcn4 in the cell is regulated primarily 
by a translational control (Albrecht et al., 1998), there is an additional amino acid starvation induced auto-activation present in filamentous fungi like Aspergillus nidulans (Hoffmann et al., 2001). In V. longisporum, Vlaro2 silencing induced the expression of the gene for amino acid cross pathway control, VlCPC1 during saprophytic life (Singh et al., 2010). This suggests that there is a similar auto-activation of $V l C P C l$ as earlier described for A. nidulans cpcA. However in $V$. longisporum only little is known about the function of cross pathway control and its possible contribution in pathogenicity of the fungus. Throughout its life cycle the pathogen is limited to two basic sources, one is the soil and the other one is the host plant. During colonization and infection $V$. longisporum occupies the xylem vessels of the oilseed crop B. napus. The xylem sap probably provides nutrition to $V$. longisporum and it may also contain certain factors such as phytohormones for the plant defence against $V$. longisporum. The xylem sap is the medium for cross-talk between the pathogen, $V$. longisporum and its host, B. napus. The composition of xylem sap consists of water, inorganic compounds, amino acids and organic acids (Sakuta et al., 1998; Kehr et al., 2005). Therefore, the effect of the xylem sap of its host, rapeseed on $V$. longisporum growth was observed in plate-based growth assays.

A reduced activity of the Vlaro 2 isogenes encoding the aromatic amino acid biosynthetic enzyme chorismate synthase resulted in reduced pathogenicity and suggested that the xylem sap does not provide sufficient amounts or well-balanced supply of aromatic amino acids (Singh et al., 2010). In this study, we present a $V$. longisporum mutant strain which has an intact basal transcription of amino acid biosynthetic genes. The strain is deficient in the transcriptional control of amino acid biosynthetic genes, which allows an increased transcription during imbalanced supply of amino acids. This mutant strain is significantly reduced in pathogenicity because VICPCl is important for successful colonization of the plant host hypocotyl. VlCPC1 seems to aid in the growth of the fungus in the xylem vessels where amino acids are scarce. Acquiring nutrient sources from its host is crucial for a fungal pathogen (Gibson et al., 2011; King et al., 2011).

\subsection{Cross pathway control is required for pathogenicity of $V$. longisporum}

In this work, we present a $V$. longisporum mutant strain, which is significantly reduced in pathogenicity. This strain shows the role cross pathway contol VlCPCl plays for pathogenicity of $V$. longisporum and successful colonization of its host. In previous studies VlCPCl shown to be induced under tryptophan starvation conditions in the wild type (Singh 
et al., 2010), VlCPC1 was similarly activated in the wild type and in a vlaro2 silenced mutant during infection of the host plants B. napus and A. thaliana (Singh et al., 2010). Assuming the fungus responds by increased VlCPCl expression to amino acid imbalance or starvation (Singh et al., 2010). Here we can actually prove the role $C P C l$ play for the plant pathogen $V$. longisporum. VlCPCl seems to aid in the growth of the fungus in the xylem vessels where amino acids are scarce. The mutant strain silenced in $\mathrm{CPCl}$ is strongly inhibited in pathogenicity, as it is no longer able to infect B. napus like the wild type. Furthermore the fungus does not reach the upper parts of the plants in later phases of infection. It is able to infect the plants, but plants infected by the silenced mutant are considerable stronger, and look healthier than plants infected by wild type strain. Measuring the fungal DNA concentration revealed, that the amounts of DNA for mutant and wild type varied strongly for different plant tissues, especially hypocotyls, stems and leaves showed significantly reduced amounts of DNA for the mutant compared to $V$. longisporum wild type strain. From height measurement of the infected plants a clear difference between wild type and $C P C 1$ silenced mutants could be observed. There is a difference of nearly $10 \mathrm{~cm}$ in height, which is very close to the mock infected plants. But still the distance of the other two mutants compared to the wild type infected plants is still significant. This supports the role of CPCl for pathogenity in $V$. longisporum.

In addition to its role as amino acid regulator, the cross-pathway control regulator Gen4/CpcA/Cpc-1 has various distinct morphological and pathogenic functions in fungi. Gcn4 controls starvation induced adhesive growth in the yeast S. cerevisiae (Braus et al., 2003; Valerius et al., 2007), which is required for pseudohyphal development. In A. nidulans, cross pathway control is connected to cellular differentiation as increase in activity of the CpcA resulted in a reversible block of fruit body formation (Hoffmann et al., 2000). Histidine starvation in C. albicans induced morphogenetic responses dependent on the global regulator, CaGen4 (Tripathi et al., 2002). In A. fumigatus, mutants impaired in cross pathway control system had strongly reduced pathogenicity and $C p c A$ seemed to be involved in establishing pulmonary aspergillosis effectively (Krappmann et al., 2004; Sasse et al., 2008; Abad et al., 2010). It will be interesting to see whether VICPCl might have an additional important role in the secondary metabolism of $V$. longisporum. In several publications (Abad et al., 2010; Elliott et al., 2011) the role of CPCl on secondary metabolism has been analysed. For Leptosphaeria maculans silencing of $C p c A$ gene resulted in a reduced production of the toxin sirodesmin (Elliott et al., 2011). In A. nidulans there is a link between CpcA and synthesis of penicillin (Busch et al., 2003). For V. longisporum we might consider there is an involvement 
in secondary metabolism of this fungus, especially when the fungus is colonizing B. napus. Inside its host such substances or metabolites might benefit the survival of the pathogen or might interphere with the defense mechanism of the plant (Fu and Wang, 2011). Other pathogens have developt strategies to counteract plant host defense (Hemetsberger et al., 2012; Kulye et al., 2012; Stergiopoulos et al., 2012; Underwood, 2012).

\subsubsection{Knockouts of $C P C 1$ in $V$. dahliae show similar effects than silencing in}

\section{V. longisporum}

Because silencing of $C P C 1$ in $V$. longisporum did not show a phenotype we decided in parallel to silencing to delete $C P C l$ in $V$. dahliae. This resulted in a deletion strain of cross pathway control that behaved similar to $V l C P C 1 \mathrm{sm}$ but with stronger effects towards amino acid starvation conditions. In fact the mutant could not grow on CDM plates supplemented with 5 MT, however certain stress conditions like cell wall stress did not affect the growth behavior or the phenotype. The deletion strain was also limited in infection of its host plant $S$. lycopersicum. In Previous studies deletions of $C P C 1$ or $C p c A$ were analyzed in different pathogenic and non pathogenic fungi (Carsiotis et al., 1974; Hinnebusch, 1984; Davis, 2000; Piotrowska, 1980; Sachs, 1996; Hoffmann et al., 2001; Krappmann et al., 2004). In the opportunistic pathogen A. fumigatus this system is required for full virulence (Krappmann et $a l ., 2004)$. In plant pathogenic fungi it was anticipated, that a knock-down or knock-out of cpcl might reduce pathogenicity (Shen and Ebbole, 1997), but was so far not proven. In A. fumigatus, mutants impaired in cross-pathway control system had strongly reduced pathogenicity and $C p c A$ seemed to be required for establishment of pulmonary aspergillosis effectively (Krappmann et al., 2004; Sasse et al., 2008; Abad et al., 2010).

\subsection{Efficiency and relieability of the silencing system}

Silencing strategy for $V$. longisporum is a reliable system for investigating the pathogenicity of the fungus. The rapeseed pathogen $V$. longisporum is a near diploid fungus with a complex genome. The nuclear DNA content of this pathogen is almost double (Karapapa et al., 1997; Collins et al., 2003). For $V$. longisporum due to the fact that for most genes tow isogenes exist (Singh et al., 2010; Inderbitzin et al., 2011; Singh et al., 2012), strategies for knock-outs are difficult to apply, especially when dealing with an almost diploid genome of this fungus. Therefore, gene silencing seems to be the optimal approach for investigating gene function. In several aproaches it was proven to be functional in V. longisporum (Singh et al., 2010; 
Singh et al., 2012) and for our investigation it also was working with high efficiency. However the efficiency of this method is not yet fully investigated for this fungus. In addition, generation of silencing constructs based on cloning techniques is very timeconsuming and not feasible for broad investigation of several genes at the same time. A particular disadvantage of silencing is that it is usually not absolute because residual activity of the gene remains. This strategy has the advantage to explore the function of genes under conditions where they are essential and where small amounts of gene expression are sufficient to keep the organism viable.

\subsection{Secondary metabolism of $V$. longisporum}

Interactions of soil-borne vascular fungi and plants include an exchange of signals within the vascular tissue. In the interaction between $V$. longisporum and B. napus, both the pathogen and the host plant are supposed to release metabolites into the xylem which affect the other partner.

Infection of the host causes symptoms which indicate the influence of secondary metabolites secreted by the pathogen, acting as elicitor-like and phytohormonal effects, suppressors of plant defense, or as virulence factors with phytotoxic effects, as it has been reported for other Verticillium spp. Verticillium spp. generate a broad spectrum of phytotoxins and elicitor compounds which induce pathogen-specific symptoms in the absence of the fungus (Nachmias et al. 1987; Buchner et al. 1989; Meyer et al. 1994; Mansoori et al. 1995). A glycoprotein elicitor purified from $V$. dahliae culture fluid was found to trigger the synthesis of phytoalexins and oxidative burst (Davis et al. 1998). Recently, VdNep (belonging to Nep1-like proteins) was isolated from $V$. dahliae and suggested to play a role as elicitor (Wang et al. 2004). Verticillium spp. utilizes cell-wall-degrading enzymes for systemic host colonization (Durrands and Cooper, 1988). Particularly pectinolytic enzymes have been shown to be important for fungal virulence on the host (Carder et al. 1987) enabling Verticillium spp. to overcome pectin-containing pit membranes between xylem elements and at vessel ends (Pegg et al. 1976; Bishop and Cooper 1983).

Disease phenotypes like stunting and premature senescence indicate interference of normal phytohormone function in relation to systemic infection and spread of the fungus (Pegg and Brady 2002). The elevation of ethylene production after Verticillium infection (Pegg and Cronshaw 1976) is associated with the development of disease symptoms and was found to be involved in disease resistance of A. thaliana (Veronese et al. 2003; Tjamos et al. 2005; 
Johansson et al. 2006). Elicitation of ethylene was referred to Verticillium phytotoxins (Mansoori and Smith 2005). Another group of potential virulence factors represent suppressors of the host defense. In tomato cultivars infected with V. albo-atrum or V. dahliae, the pathogen can suppress the activity of phenylalanine ammonia-lyase (PAL) (Lee et al. 1992; Gold and Robb 1995), an essential enzyme which is involved in the synthesis of suberin and lignin (Hahlbrock and Scheel 1989) but also regulates the synthesis of salicylic acid (SA). Suppression of PAL activity results in less suberin coating in the xylem which is one part of the plant defense response (Lee et al. 1992).

The host specificity of many fungi is often determined by whether or not the pathogen has the enzymes to detoxify a particular plant defense product (van Etten et al. 1989; Osbourn 1999).

\subsection{LAE1 in V. longisporum and $V$. dahliae}

It could be demonstrated that amino acid metabolism is required for infection and colonization in $V$. longisporum as well as $V$. dahliae. In a second approach we tried to investigate the role of secondary metabolism in Verticilliae. Therefore we decided to observe the role of LAE1, which is the key regulator of secondary metabolism in several fungi (Keller and Hohn, 1997; Keller et al., 2005; Bayram et al., 2008; Bayram and Braus, 2012). A knock out of LAE1 did not show an effect on pathogenicity of $V$. dahliae, while silencing of this gene in $V$. longisporum revealed stronger effects in pathogenicity. Silencing of genes, especially genes with conserved domains can lead to co-silencing of genes with similar domains (Nakayashiki, 2005). To analyze whether other methyl tranferase containing genes might be co-silenced by the silencing construct for $L A E 1$, an alignment of similar potential genes in $V$. longisporum with the sequence of the silencing construct was performed (Fig. 45). The two hosts chosen for plant infection differ in their behavior after infection. Infected plants of B. napus shows very strong effects, and differences between infected and non infected plants are clearly visible. Infections with $V$. dahliae on tomato show milder symptoms and the difference between infected and non infected plants is much less pronounced. 


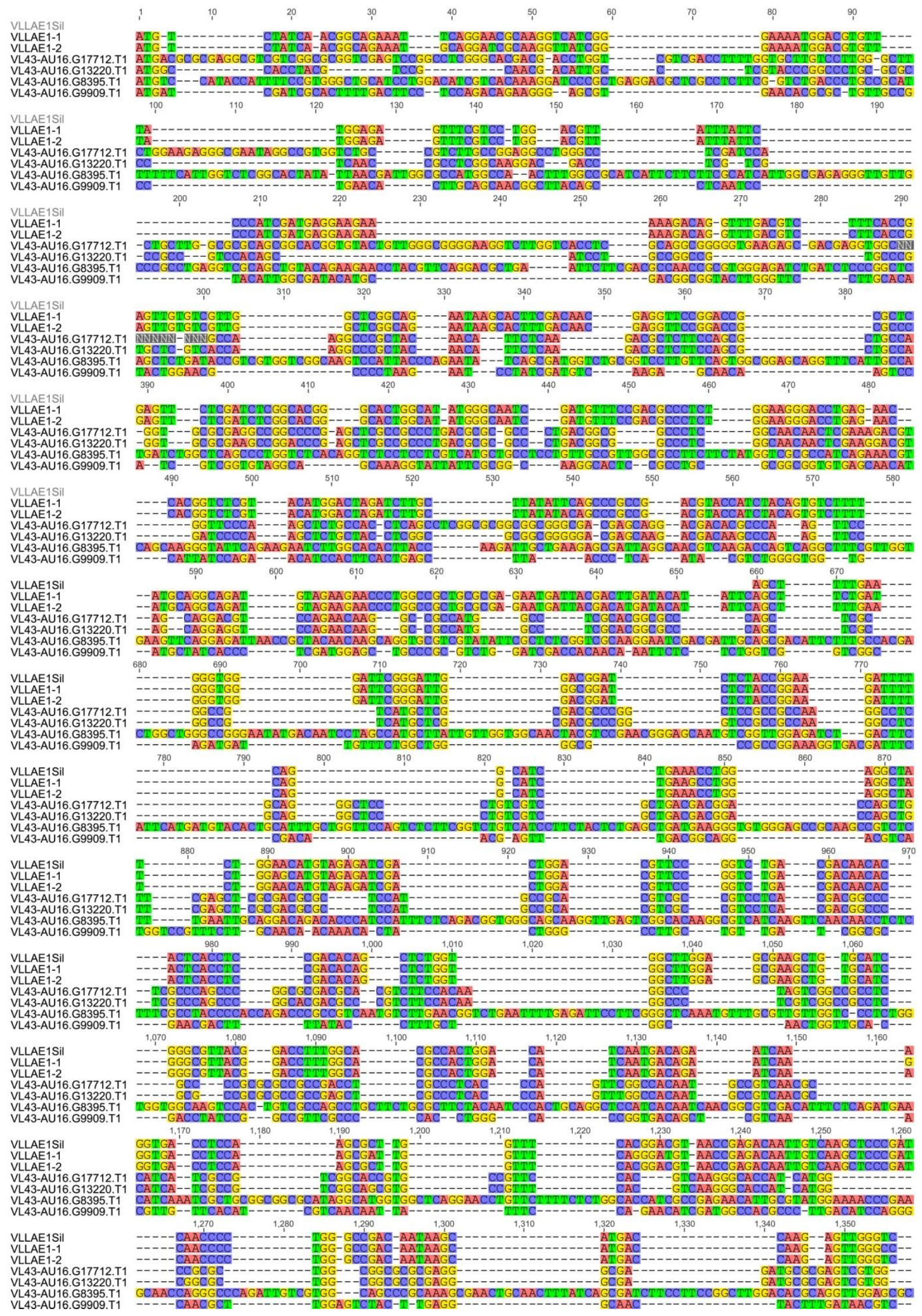

Fig. 45 Sequence alignment of $L A E 1$ sequences. Alignments of the deduced nucleotide sequences of LAE1 of $V$. longisporum in comparison to methyl transferase domain containing genes. Colors indicate different nucleotides. 
The sequence of the silencing construct could align with several other genes containing methyl transferase domains. There is a possibility that these genes might be co-silenced by the $L A E 1$ silencing construct.

As mentioned for $\mathrm{CPCl}$ earlier the efficiency of this method is not yet fully investigated for this fungus. In addition, generation of silencing constructs based on cloning techniques is very time-consuming and not feasible for broad investigation of several genes at the same time. A particular disadvantage of silencing is that it is usually not absolute because residual activity of the gene remains. For the silencing of LAE1 there might be an additional problem. As shown in Fig. 44 there are several other sequences beside the target sequence which might interact with the hairpin construct for silencing and be co-silenced by this construct this effect might alter the pathogenicity of the mutant and influence the behaviour inside the plant. All these factors have to be considered when analysing these data.

$L A E 1$ silencing in $V$. longisporum revealed an effect on pathogenicity. In plant infection the silencing had an effect on the pathogenicity of the fungus. The disease score for VILAE1sm is lower than for $V$. longisporum wild type. Compared to $V l C P C l \mathrm{sm}$ the effect is not as strong but still clear. The mutant strain silenced in $L A E 1$ is inhibited in pathogenicity. He is capable of infecting its host B. napus, but regarding wild type infection a decrease of infection level could be observed. The plants infected with the silenced mutant are considerable stronger, and look healthier than wild type infected plants. The same effect can be investigated on DNA levels. The fungal DNA concentration revealed, that the amounts of DNA for mutant and wild type varied for different plant tissues, especially hypocotyls, stems and leaves showed significantly reduced amounts of DNA for the mutant compared to $V$. longisporum wild type strain. The silenced mutants show a reduction of roughly $1 / 3$ on DNA levels in hypocotyls, stems and leaves. Height measurement of the infected plants also support a clear difference between wild type and LAE1 silenced mutants. But reduced pathogenicity of the mutant must not correspond with a reduced production of secondary metabolites. We could prove that the gene for $L A E 1$ is silenced in $V$. longisporum. And for $V$. longisporum we could observe an effect on pathogenicity.

\subsubsection{LAE1 in V. dahliae}

From the results we received by analysis of $L A E 1$ silencing we anticipated similar effects for a knockout in $V$. dahliae. A successful deletion of LAEl was established in $V$. dahliae. The 
mutant did not show any effect on pathogenicity of the fungus. Which might have several reason. Like mentioned above silencing of $L A E 1$ might have affected some other gene with homology in the sequence which was chosen for silencing, and therefore were co-silenced by the silencing construct. This might give the data we aquired for LAE1 in V. longisporum another meaning. One of the co-silenced genes might as well be involved in the pathogenicity of the fungus. This had an influence on the growth behavior of the fungus in the plant. The silencing construct is randomly integrated into the genome of the fungus. There is a possibility that the construct is integrated into the position of a gene locus and therefore has disrupted the function of a pathogenicity related gene. Another thing is the difference between the hosts chosen for plant infection. Infection of B. napus shows very strong effects, the differences between infected and non infected plants are clearly visible. While tomato infections show milder symptoms. The observed effects are still visible but less pronounced than in oilseed rape. The average difference between infected and non infected plants in tomatoe is much smaller than the difference between infected and non infected oilseed rape plants. This might be another reason for the why the effects of these plants are easier to notice than those of the tomatoe plants.

It might also be the reason of a different gene function of $L A E 1$ in V. dahliae. In Trichoderma reesei it was demonstrated, that $L A E 1$ of this fungus has a contrasting role (Karimi-Anghcheh et al., 2013).

Futhermore $L A E 1$ might not play a crucial role for disease development and pathogenicity in $V$. dahliae. The infection of the plants is mainly limited to the xylem vessels of the plant (Zhou et al., 2006; Eynck et al., 2007), where LAE1 might not essentially be required for colonization of the plants. Other factors might play a role under these enviroment conditions.

\subsection{Triacylglycerol lipase}

Successful infection and colonization of the host requires proteins or enzymes that are capable of acquiring nutrition for the fungal pathogen, or provide opportunities of breaking the plant cell wall. Secreted proteins and secondary metabolites are the key players for these reactions. These proteins or enzymes might be useful to adapt to the plants pathogen reaction or either might be important to counteract the pathogen response of the host plant. Among these enzymes, lipases and cutinases, with their potential roles in the hydrolyzation of the plant cuticle and surface waxes, may contribute to pathogen infection at an early stage, when the fungus first comes into contact with its host. Here we could demonstrate with 
triacylglycerol lipase $(T A G L)$ as an example that their might be a role for breaking the plant cell well and enter the host. The lipase was originally detected in a protein assay were $V$. longisporum cultures were treated with xylem sap. Due to this treatment the lipase was upregulated significantly and was chosen as a candidate for further investigastion.

Silencing of TAGL in $V$. longisporum showed reduced pathogenicity. It might be considered to play a role for infection of the plant. The plants are lesser affected by infection with the fungus. Compared to CPC1 silenced mutants, the effects were less pronounced but still clearly visible in its host $B$. napus. The lipase itself could be required for breaking the plantal cell wall like discribed for other phytopathogens (Voigt et al., 2005). The cutinase activity of this lipase might be involved in breaking the cell wall of the plant.

Regarding the lipase was induced after treatment with xylem sap it gives a strong hint that it might be required inside the plant for several functions. It might provide nutritiens to the fungus. For further investigation a knockout in $V$. dahliae would be interesting. Maybe a knockout would show stronger effects on pathogenicity. The fungus might even not be able to enter its host because the required enzyme is not available. Another test would be for lipase activity which was shown in other studies (Voigt et al., 2005). Clearly a knock out of this gene in $V$. dahliae and plant infection assays to investigate this candidate gene in more detail is neccessary.

\subsection{Conclusions and Outlook}

Pathogenic organisms like the filamentous fungi A. fumigatus require pathways and mechanisms for adaptation within the host. A better understanding of these regulatory systems make easier to find new targets for antifungal drugs and pharmaceutica. One of these systems is the cross pathway control, which is the homologous to the well-studied General Control of amino acid biosynthesis (GC) (Hannig and Hinnebusch, 1988; Hannig et al., 1990; Hinnebusch, 2005). In its core, this global regulatory system contains a sensor kinase (CpcC) and a transcriptional regulator (CpcA) (Hoffmann et al., 2001), for which it was demonstrated that it is required for full virulence of A. fumigatus (Krappmann et al., 2004). Therefore, the main focus of this work was set on the regulatory gene $C P C 1$. Understanding of this regulatory mechanism enables a more detail insight of a pathogenic filamentous fungus resulting in increasing knowledge that can give hints for new treatments against this plant pathogen. 
$V$. longisporum is pathogenic on oilseed rape and it colonizes the xylem vessels of the plant in its biotrophic phase in the plant. The xylem sap contains water, inorganic minerals, some organic compounds and also amino acids. The fungus is dependent on the xylem sap for its nutrient supply in the plant. $\mathrm{Cpc}$ is the global regulator of amino acid biosynthesis and many secondary metabolites produced in fungi are depending on amino acids. The goal of this study was to find out whether $C P C l$ has an influence on pathogenicity of $V$. longisporum in the rapeseed. Therefore, we chose to silence the isogenes of $C P C 1$. In parallel we also performed a deletion of the gene in $V$. dahliae to whether there is a difference between silencing and deletion of a gene. In $V$. longisporum silcencing of cross pathway control had no influence on growth morphology of the fungus, but lead to a mutant which is sensitive to amino acid starvation conditions. This handicap does not have an influence on normal growth conditions of the pathogen, though when restricted in amino acids the growth rate of the silenced mutant was strongly affected. In plant infection assays with limited supply of amino acids the fungus is significantly reduced in growth and infection rate. Under normal conditions $V$. longisporum spreads throughout the plant after 28 days post infection and becomes detectable via RT-PCR in the upper parts of the plants in significant amounts. For VlCPClsm only 40-50\% of DNA amounts are detectable in the stem and leaves compared to wild type.

LAE1 silencing in $V$. longisporum revealed an effect on pathogenicity. But reduced pathogenicity of the mutant must not correspond with a reduced production of secondary metabolites. We could prove that the gene for $L A E 1$ is silenced in $V$. longisporum. For further characterization of metabolites a reliable database must be established. Furthermore it requires also some basic experiments were certain gene clusters for secondary metabolism are activated and certain products can be identified. In addition the silencing of the lipase revealed an effect on the pathogenicity which needs to be further analysed. Regarding the lipase was induced after treatment with xylem sap it gives a strong hint that it might be required inside the plant for several functions. It might provide nutritiens to the fungus. Clearly a knock out of this gene in $V$. dahliae and plant infection assays to investigate this candidate gene in more detail is neccessary.

In summary we conclude that the regulation of cross pathway control by Cpc1 plays an important role for the Verticillium pathogens to counteract the imbalanced amino acid supply inside the plant xylem vessels. This is the first time we report $\mathrm{Cpc1}$ is required for plant pathogens to cause disease. Cpc1 appears to be a factor for communication between the fungi and the limited enviroment of amino acids provided by the plant. Furthermore we 
investigated roles in pathogenicity of a key regulator LaeA/Lae1 of secondary metabolism and a putative secreted lipase with a conserved cutinase domain in Verticillium. Silencing of the candidate genes in $V$. longisporum resulted in reduced pathogenicity. However deletion of $L A E 1$ in $V$. dahliae did not lead to similar results like LAE1 silencing in $V$. longisporum. The role of Lae1 for secondary metabolite production remains characterized. The secreted lipase seem to be an interresting candidate for further investigation. Therefore we suggest that potential function of the lipase gene in pathogenicity needs to be confirmed by gene deletion than only gene silencing. 


\section{References}

Abad, A., Fernandez-Molina, J.V., Bikandi, J., Ramirez, A., Margareto, J., Sendino, J., Hernando, F.L., Ponton, J., Garaizar, J., and Rementeria, A. 2010. What makes Aspergillus fumigatus a successful pathogen? Genes and molecules involved in invasive aspergillosis. Rev Iberoam Micol 27:155-182.

Agrios, G. 2005. Plant pathology, 5th. Elsevier Academic Press, USA.

Albrecht, G., Mösch, H.U., Hoffmann, B., Reusser, U., and Braus, G.H. 1998. Monitoring the Gcn 4 protein-mediated response in the yeast Saccharomyces cerevisiae. J Biol Chem 273:12696-12702.

Amaike, S., and Keller, N.P. 2009. Distinct roles for VeA and LaeA in development and pathogenesis of Aspergillus flavus. Eukaryot Cell 8:1051-1060.

Arndt, K., and Fink, G.R. 1986. GCN4 protein, a positive transcription factor in yeast, binds general control promoters at all 5' TGACTC 3' sequences. Proc Natl Acad Sci U S A 83:8516-8520.

Babadoost, M., Chen, W., Bratsch, A.D., and Eastman, C.E. 2004. Verticillium longisporum and Fusarium solani: two new species in the complex of internal discoloration of horseradish roots. Plant Pathol. 53:669-676.

Barbara, D.J., and Clewes, E. 2003. Plant pathogenic Verticillium species: how many of them are there? Mol Plant Pathol 4:297-305.

Barbara, D.J., Morton, A., and Miller, N.J. 2005. Isolation of microsatellite markers from an interspecific hybrid isolate of the fungal plant pathogen Verticillium dahliae. Mol Ecol Notes 5:854-856.

Bayram, O., and Braus, G.H. 2012. Coordination of secondary metabolism and development in fungi: the velvet family of regulatory proteins. FEMS Microbiol Rev 36:1-24.

Bayram, O., Krappmann, S., Ni, M., Bok, J.W., Helmstaedt, K., Valerius, O., BrausStromeyer, S., Kwon, N.J., Keller, N.P., Yu, J.H., and Braus, G.H. 2008. VelB/VeA/LaeA complex coordinates light signal with fungal development and secondary metabolism. Science 320:1504-1506.

Beckman, C.H. 1987. The nature of wilt diseases of plants. The American Phytopathological Society, St Paul, MN.

Berlanger, I., and Powelson, M.L. 2000. Verticillium wilt. The Plant Health Instructor. Doi: 10.1094/PHI-I-PHI-I-2000-0801-01.

Bhat, R.G., and Subbarao, K.V. 1999. Host Range Specificity in Verticillium dahliae. Phytopathology 89:1218-1225.

Bok, J.W., and Keller, N.P. 2004. LaeA, a regulator of secondary metabolism in Aspergillus spp. Eukaryot Cell 3:527-535.

Bok, J.W., Noordermeer, D., Kale, S.P., and Keller, N.P. 2006. Secondary metabolic gene cluster silencing in Aspergillus nidulans. Mol Microbiol 61:1636-1645.

Bok, J.W., Balajee, S.A., Marr, K.A., Andes, D., Nielsen, K.F., Frisvad, J.C., and Keller, N.P. 2005. LaeA, a regulator of morphogenetic fungal virulence factors. Eukaryot Cell 4:1574-1582.

Braus, G.H., Grundmann, O., Brückner, S., and Mösch, H.U. 2003. Amino acid starvation and Gcn $4 p$ regulate adhesive growth and FLO11 gene expression in Saccharomyces cerevisiae. Mol Biol Cell 14:4272-4284.

Brown, D.W., Yu, J.H., Kelkar, H.S., Fernandes, M., Nesbitt, T.C., Keller, N.P., Adams, T.H., and Leonard, T.J. 1996. Twenty-five coregulated transcripts define a 
sterigmatocystin gene cluster in Aspergillus nidulans. Proc Natl Acad Sci U S A 93:1418-1422.

Busch, S., Bode, H.B., Brakhage, A.A., and Braus, G.H. 2003. Impact of the cross-pathway control on the regulation of lysine and penicillin biosynthesis in Aspergillus nidulans. Curr Genet 42:209-219.

Calvo, A.M., Wilson, R.A., Bok, J.W., and Keller, N.P. 2002. Relationship between secondary metabolism and fungal development. Microbiol Mol Biol Rev 66:447-459.

Carsiotis, M., Jones, R.F., and Wesseling, A.C. 1974. Cross-pathway regulation: histidinemediated control of histidine, tryptophan, and arginine biosynthetic enzymes in Neurospora crassa. J Bacteriol 119:893-898.

Chen, P., Lee, B., and Robb, J. 2004. Tolerance to a non-host isolate of Verticillium dahliae in tomato. Physiol. Mol. Plant Pathol 64:283-291.

Clewes, E., and Barbara, D.J. 2005. Hopeful monsters. In: Program and Book of Abstracts of the Ninth International Verticillium Symposium. California, USA, 17-21 June, p.18.

Clewes, E., and Barbara, D.J. 2008. Two allopolyploid ascomycete fungal plant pathogens were not rescued by vertical transmission. New Phytol 177:583-585.

Collado-Romero, M., Jiménez-Díaz, R.M., and Mercado-Blanco, J. 2010. DNA sequence analysis of conserved genes reveals hybridization events that increase genetic diversity in Verticillium dahliae. Fungal Biol. 114:209-218.

Collins, A., Okoli, C.A., Morton, A., Parry, D., Edwards, S.G., and Barbara, D.J. 2003. Isolates of Verticillium dahliae Pathogenic to Crucifers Are of at Least Three Distinct Molecular Types. Phytopathology 93:364-376.

Cox, R.J. 2007. The biosynthesis of polyketides, acyl tetramic acids and pyridones by filamentous fungi. In: Exploitation of Fungi Eds Robson GD, van West P \& Gadd GM CUP.

Davis, R.H. 2000. Neurospora: Contributions of a Model Organism. Oxford, England:Oxford University Press.

Dixelius, C., Happstadius, I., and Berg, G. 2005. Verticillium wilt on Brassica oil crops - a swedish perspective. J. Swedish Seed Association 115:36-48.

Dobinson, K.F., Grant, S.J., and Kang, S. 2004. Cloning and targeted disruption, via Agrobacterium tumefaciens-mediated transformation, of a trypsin protease gene from the vascular wilt fungus Verticillium dahliae. Curr Genet 45:104-110.

Drysdale, C.M., Jackson, B.M., McVeigh, R., Klebanow, E.R., Bai, Y., Kokubo, T., Swanson, M., Nakatani, Y., Weil, P.A., and Hinnebusch, A.G. 1998. The Gcn4p activation domain interacts specifically in vitro with RNA polymerase II holoenzyme, TFIID, and the Adap-Gen5p coactivator complex. Mol Cell Biol 18:1711-1724.

Dunker, S., Keunecke, H., and von Tiedemann, A. 2006. Verticillium longisporum in winter oilseed rape-Impact on plant development and yield. Integr Control Oilseed Crops 29:365-374.

Eastburn, D.M., and Chang, R.J. 1994. Verticillium dahliae: A causal agent of root discoloration of horseradish in Illinois. Plant Dis. 78:496-498.

Ehrt, S., and Schnappinger, D. 2003. Isolation of plasmids from E. coli by boiling lysis. Methods Mol Biol 235:79-82.

Ellenberger, T.E., Brandl, C.J., Struhl, K., and Harrison, S.C. 1992. The GCN4 basic region leucine zipper binds DNA as a dimer of uninterrupted alpha helices: crystal structure of the protein-DNA complex. Cell 71:1223-1237.

Elliott, C.E., Fox, E.M., Jarvis, R.S., and Howlett, B.J. 2011. The cross-pathway control system regulates production of the secondary metabolite toxin, sirodesmin PL, in the ascomycete, Leptosphaeria maculans. BMC Microbiol 11:169. 
Eynck, C., Koopmann, B., Karlovsky, P., and von Tiedemann, A. 2009. Internal resistance in winter oilseed rape inhibits systemic spread of the vascular pathogen Verticillium longisporum. Phytopathology 99:802-811.

Eynck, C., Koopmann, B., Grunewaldt-Stoecker, G., Karlovsky, P., and von Tiedemann, A. 2007. Differential interactions of Verticillium longisporum and V. dahliae with Brassica napus detected with molecular and histological techniques. Eur J Plant Pathol 118:259-274.

Fahleson, J., Hu, Q., and Dixelius, C. 2004. Phylogenetic analysis of Verticillium species based on nuclear and mitochondrial sequences. Arch Microbiol 181:435-442.

Fernandes, M., Keller, N.P., and Adams, T.H. 1998. Sequence-specific binding by Aspergillus nidulans AfIR, a C6 zinc cluster protein regulating mycotoxin biosynthesis. Mol Microbiol 28:1355-1365.

Floerl, S., Majcherczyk, A., Possienke, M., Feussner, K., Tappe, H., Gatz, C., Feussner, I., Kues, U., and Polle, A. 2012. Verticillium longisporum infection affects the leaf apoplastic proteome, metabolome, and cell wall properties in Arabidopsis thaliana. PLoS One 7:e31435.

Fradin, E.F., and Thomma, B.P.H.J. 2006. Physiology and molecular aspects of Verticillium wilt diseases caused by $V$. dahliae and V. albo-atrum. Mol. Plant. Pathol 7:71-86.

Fradin, E.F., Zhang, Z., Juarez Ayala, J.C., Castroverde, C.D., Nazar, R.N., Robb, J., Liu, C.M., and Thomma, B.P. 2009. Genetic dissection of Verticillium wilt resistance mediated by tomato Ve1. Plant Physiol 150:320-332.

$\mathrm{Fu}$, J., and Wang, S. 2011. Insights into auxin signaling in plant-pathogen interactions. Front Plant Sci 2:74.

Garas, N.A., Wilhelm, S., and Sagen, J.E. 1986. Relationship of cultivar resistance to distribution of Verticillium dahliae in inoculated cotton plants and to growth of single conidia on excised stem segments. Phytopathology 76:1005-1010.

Gibson, D.M., King, B.C., Hayes, M.L., and Bergstrom, G.C. 2011. Plant pathogens as a source of diverse enzymes for lignocellulose digestion. Curr Opin Microbiol 14:264270.

Gladders, P., Smith, J.A., Kirkpatrick, L., Clewes, E., Grant, C., Barbara, D., Barnes, A.V., and Lane, C.R. 2011. First record of Verticillium wilt (Verticillium longisporum) in winter oilseed rape in the UK. New Dis. Rep 23.

Gold, J., and Robb, J. 1995. The role of the coating response in Craigella tomatoes infected with Verticillium dahliae, races 1 and 2. Physiological and Molecular Plant Pathology 47:141-157.

Goud, J.K., Termorshuizen, A.J., and Gams, W. 2003. Morphology of Verticillium dahliae and $V$. tricorpus on semi-selective media used for the detection of $V$. dahliae in soil. Mycol Res 107:822-830.

Griffiths, D.A. 1982. Structure of Verticillium nigresgens and V. nubilum chlamydospores. Trans. Br. Mycol. Soc. 78(1):141-145.

Grundmann, O., Mösch, H.U., and Braus, G.H. 2001. Repression of GCN4 mRNA translation by nitrogen starvation in Saccharomyces cerevisiae. J Biol Chem 276:25661-25671.

Hannig, E.M., and Hinnebusch, A.G. 1988. Molecular analysis of GCN3, a translational activator of GCN4: evidence for posttranslational control of GCN3 regulatory function. Mol Cell Biol 8:4808-4820.

Hannig, E.M., Williams, N.P., Wek, R.C., and Hinnebusch, A.G. 1990. The translational activator GCN3 functions downstream from GCN1 and GCN2 in the regulatory pathway that couples GCN4 expression to amino acid availability in Saccharomyces cerevisiae. Genetics 126:549-562. 
Hastie, A.C. 1973. Hybridization of Verticillium albo-atrum and Verticillium dahliae. Trans. Br. Mycol. Soc 60 (3):511-523.

Heale, J.B., and Karapapa, V.K. 1999. The Verticillium threat to Canada's major oilseed crop: Canola. Canadian Journal of Plant Pathology 21:1-7.

Heinz, R., Lee, S.W., Saparno, A., Nazar, R.N., and Robb, J. 1998. Cyclical systemic colonization in Verticillium-infected tomato. Physiol Mol Plant Patholog 52.

Hemetsberger, C., Herrberger, C., Zechmann, B., Hillmer, M., and Doehlemann, G. 2012. The Ustilago maydis Effector Pep1 Suppresses Plant Immunity by Inhibition of Host Peroxidase Activity. PLoS Pathog 8:e1002684.

Hinnebusch, A.G. 1984. Evidence for translational regulation of the activator of general amino acid control in yeast. Proc Natl Acad Sci U S A 81:6442-6446.

Hinnebusch, A.G. 1997. Translational regulation of yeast GCN4. A window on factors that control initiator-trna binding to the ribosome. J Biol Chem 272:21661-21664.

Hinnebusch, A.G. 2005. Translational regulation of GCN4 and the general amino acid control of yeast. Annu Rev Microbiol 59:407-450.

Hinnebusch, A.G., and Natarajan, K. 2002. Gcn4p, a master regulator of gene expression, is controlled at multiple levels by diverse signals of starvation and stress. Eukaryot Cell $1: 22-32$.

Hoffmann, B., Wanke, C., Lapaglia, S.K., and Braus, G.H. 2000. c-Jun and RACK1 homologues regulate a control point for sexual development in Aspergillus nidulans. Mol Microbiol 37:28-41.

Hoffmann, B., Valerius, O., Andermann, M., and Braus, G.H. 2001. Transcriptional autoregulation and inhibition of mRNA translation of amino acid regulator gene cpcA of filamentous fungus Aspergillus nidulans. Mol Biol Cell 12:2846-2857.

Howlett, B.J. 2006. Secondary metabolite toxins and nutrition of plant pathogenic fungi. Curr Opin Plant Biol 9:371-375.

Inderbitzin, P., Davis, R.M., Bostock, R.M., and Subbarao, K.V. 2011. The ascomycete Verticillium longisporum is a hybrid and a plant pathogen with an expanded host range. PLoS One 6:e18260.

Ingram, R. 1968. Verticillium dahliae Kleb. var. longisporum Stark: a stable diploid. Trans. Br. Mycol. Soc. 51:339-341.

Isaac, I. 1947. A comparative study of pathogenic isolates of Verticillium. Trans. Br. Mycol. Soc. 32:137-157.

Isaac, I., and Milton, J.M. 1967. Response of Verticillium species to griseofulvin. J Gen Microbiol 46:273-283.

Iven, T., König, S., Singh, S., Braus-Stromeyer, S.A., Bischoff, M., Tietze, L.F., Braus, G.H., Lipka, V., Feussner, I., and Dröge-Laser, W. 2012. Transcriptional Activation and Production of Tryptophan-Derived Secondary Metabolites in Arabidopsis Roots Contributes to the Defense against the Fungal Vascular Pathogen Verticillium longisporum. Mol Plant.

Janus, D., Hoff, B., Hofmann, E., and Kück, U. 2007. An efficient fungal RNA-silencing system using the DsRed reporter gene. Appl Environ Microbiol 73:962-970.

Johannson, A., Goud, J.-K., and Dixelius, C. 2005. Plant host range of Verticillium longisporum and microsclerotia density in Swedish soils. . Eur J Plant Pathol 114:139-149.

Johannson, A., Fahleson, J., Zhou, L., Hu, Q., and Dixelius, C. 2003. Genomic characterisation of Verticillium longisporum. Eigth International Congress on Plant Pathology. Christchurch, New Zealand, 2-7 Feb 2003:347. 
Jyothishwaran, G., Kotresha, D., Selvaraj, T., Srideshikan, S.H., Rajvanshi, P.K., and Jayabaskaran, C. 2007. A modified freeze-thaw method for efficient transformation of Agrobacterium tumefaciens. Curr. Sci. 93(6):770-772.

Karapapa, V.K., B.W., B., and J.B., H. 1997. Morphological and molecular characterization of Verticillium longisporum comb. nov., pathogenic to oilseed rape. Mycol. Res. 101:1281-1293.

Karimi Aghcheh R, Druzhinina IS, Kubicek CP. 2013. The putative protein methyltransferase LAE1 of Trichoderma atroviride is a key regulator of asexual development and mycoparasitism. PLoS One 24;8(6):e67144.

Kehr, J., Buhtz, A., and Giavalisco, P. 2005. Analysis of xylem sap proteins from Brassica napus. BMC Plant Biol 5:11.

Keller, N.P., and Adams, T.H. 1995. Analysis of a mycotoxin gene cluster in Aspergillus nidulans. SAAS Bull Biochem Biotechnol 8:14-21.

Keller, N.P., and Hohn, T.M. 1997. Metabolic Pathway Gene Clusters in Filamentous Fungi. Fungal Genet Biol 21:17-29.

Keller, N.P., Turner, G., and Bennett, J.W. 2005. Fungal secondary metabolism - from biochemistry to genomics. Nat Rev Microbiol 3:937-947.

Kim, J.T., Park, I.H., Lee, H.B., Hahm, Y.I., and Yu, S.H. 2001. Identification of Verticillium dahliae and V. albo-atrum causing wilt of tomato in Korea. Plant Pathol.J. 17(4):222226.

King, B.C., Waxman, K.D., Nenni, N.V., Walker, L.P., Bergstrom, G.C., and Gibson, D.M. 2011. Arsenal of plant cell wall degrading enzymes reflects host preference among plant pathogenic fungi. Biotechnol Biofuels 4:4.

Klimes, A., and Dobinson, K.F. 2006. A hydrophobin gene, VDH1, is involved in microsclerotial development and spore viability in the plant pathogen Verticillium dahliae. Fungal Genet Biol 43:283-294.

Klosterman, S.J., Atallah, Z.K., Vallad, G.E., and Subbarao, K.V. 2009. Diversity, pathogenicity, and management of verticillium species. Annu Rev Phytopathol 47:3962.

Klosterman, S.J., Subbarao, K.V., Kang, S., Veronese, P., Gold, S.E., Thomma, B.P., Chen, Z., Henrissat, B., Lee, Y.H., Park, J., Garcia-Pedrajas, M.D., Barbara, D.J., Anchieta, A., de Jonge, R., Santhanam, P., Maruthachalam, K., Atallah, Z., Amyotte, S.G., Paz, Z., Inderbitzin, P., Hayes, R.J., Heiman, D.I., Young, S., Zeng, Q., Engels, R., Galagan, J., Cuomo, C.A., Dobinson, K.F., and Ma, L.J. 2011. Comparative genomics yields insights into niche adaptation of plant vascular wilt pathogens. PLoS Pathog 7:e1002137.

Koike, S.T., Subbarao, K.V., Davis, R.M., Gordon, T.R., and Hubbard, J.C. 1994. Verticillium ilt of cauliflower in California. Plant Disease 78:1116-1121.

Krappmann, S., Bignell, E.M., Reichard, U., Rogers, T., Haynes, K., and Braus, G.H. 2004. The Aspergillus fumigatus transcriptional activator CpcA contributes significantly to the virulence of this fungal pathogen. Mol Microbiol 52:785-799.

Kulye, M., Liu, H., Zhang, Y., Zeng, H., Yang, X., and Qiu, D. 2012. Hrip1, a novel protein elicitor from necrotrophic fungus, Alternaria tenuissima elicits cell death, expression of defense related genes, and systemic acquired resistance in tobacco. Plant Cell Environ.

Mahuku, G.S., and Platt, H.W. 2002. Molecular evidence that Verticillium albo-atrum Grp2 isolates are distinct from V. albo-atrum Grp1 and V. tricorpus. Mol.Plant Pathol. 3:71-79.

Mann, J. 1986. Secondary Metabolism. OUP, Oxford. 
Mattinen, L., Nissinen, R., Riipi, T., Kalkkinen, N., and Pirhonen, M. 2007. Host-extract induced changes in the secretome of the plant pathogenic bacterium Pectobacterium atrosepticum. Proteomics 7:3527-3537.

Mayer, A.M., Staples, R.C., and Gil-ad, N.L. 2001. Mechanisms of survival of necrotrophic fungal plant pathogens in hosts expressing the hypersensitive response. Phytochemistry 58:33-41.

Mirande, M., and Waller, J.P. 1988. The yeast lysyl-tRNA synthetase gene. Evidence for general amino acid control of its expression and domain structure of the encoded protein. J Biol Chem 263:18443-18451.

Morton, A., Carder, J.H., and Barbara, D.J. 1995. Sequences of the internal transcribed spacers of the ribosomal RNA genes and relationships between isolates of Verticillium albo-atrum and V. dahliae. Plant Pathol. 44:183-190.

Mösch, H.U., Scheier, B., Lahti, R., Mantsala, P., and Braus, G.H. 1991. Transcriptional activation of yeast nucleotide biosynthetic gene ADE4 by GCN4. J Biol Chem 266:20453-20456.

Mukherjee, S., Dawe, A.L., and Creamer, R. 2012. Potential role for saccharopine reductase in swainsonine metabolism in endophytic fungus, Undifilum oxytropis. Fungal Biol 116:902-909.

Muller, M.M., and Hausmann, R. 2011. Regulatory and metabolic network of rhamnolipid biosynthesis: traditional and advanced engineering towards biotechnological production. Appl Microbiol Biotechnol 91:251-264.

Mullins, E.D., Chen, X., Romaine, P., Raina, R., Geiser, D.M., and Kang, S. 2001. Agrobacterium-Mediated Transformation of Fusarium oxysporum: An Efficient Tool for Insertional Mutagenesis and Gene Transfer. Phytopathology 91:173-180.

Nagao, H., Wakatabe, D., and Iijima, T. 1994. Difficulty in establishing vegetative compatibility of Japanese isolates of Verticillium dahliae Kleb. using melaninsynthesis deficient mutants. J. Gen. Appl. Microbiol. 40(4):277-285.

Nakayashiki, H. 2005. RNA silencing in fungi: mechanisms and applications. FEBS Lett 579:5950-5957.

Natarajan, K., Meyer, M.R., Jackson, B.M., Slade, D., Roberts, C., Hinnebusch, A.G., and Marton, M.J. 2001. Transcriptional profiling shows that Gcn4p is a master regulator of gene expression during amino acid starvation in yeast. Mol Cell Biol 21:43474368 .

Neumann, M.J., and Dobinson, K.F. 2003. Sequence tag analysis of gene expression during pathogenic growth and microsclerotia development in the vascular wilt pathogen Verticillium dahliae. Fungal Genet Biol 38:54-62.

Okubara, P.A., and Paulitz, T.C. 2005. Root defense responses to fungal pathogens: A molecular perspective. Plant and Soil 274:215-226.

Oliphant, A.R., Brandl, C.J., and Struhl, K. 1989. Defining the sequence specificity of DNAbinding proteins by selecting binding sites from random-sequence oligonucleotides: analysis of yeast GCN4 protein. Mol Cell Biol 9:2944-2949.

Paluh, J.L., Orbach, M.J., Legerton, T.L., and Yanofsky, C. 1988. The cross-pathway control gene of Neurospora crassa, cpc-1, encodes a protein similar to GCN4 of yeast and the DNA-binding domain of the oncogene v-jun-encoded protein. Proc Natl Acad Sci U S A 85:3728-3732.

Pedley, K.F., and Walton, J.D. 2001. Regulation of cyclic peptide biosynthesis in a plant pathogenic fungus by a novel transcription factor. Proc Natl Acad Sci U S A 98:14174-14179.

Pegg, G.F., and Brady, B.L. 2002. Verticillium wilts. CABI Publishing, New York. 
Piotrowska, M., Kruszewska, A., and Paszewski, A. 1980. Effect of regulatory mutations of sulphur metabolism on the levels of cysteine- and homocysteine-synthesizing enzymes in Neurospora crassa. Acta Biochim Pol 27:395-403.

Presley, J.T., Carns, H.R., Taylor, E.E., and Schnathorst, W.C. 1966. Movement of conidia of Verticillium albo-atrum in cotton plants. Phytopathology 56:375.

Qin, Q.M., Vallad, G.E., and Subbarao, K.V. 2008. Characterization of Verticillium dahliae and $V$. tricorpus isolates from lettuce and artichoke. Plant Dis. 92:69-77.

Qin, Q.M., Vallad, G.E., Wu, B.M., and Subbarao, K.V. 2006. Phylogenetic Analyses of Phytopathogenic Isolates of Verticillium spp. Phytopathology 96:582-592.

Ratzinger, A., Riediger, N., von Tiedemann, A., and Karlovsky, P. 2009. Salicylic acid and salicylic acid glucoside in xylem sap of Brassica napus infected with Verticillium longisporum. J Plant Res 122:571-579.

Rauyaree, P., Ospina-Giraldo, M.D., Kang, S., Bhat, R.G., Subbarao, K.V., Grant, S.J., and Dobinson, K.F. 2005. Mutations in VMK1, a mitogen-activated protein kinase gene, affect microsclerotia formation and pathogenicity in Verticillium dahliae. Curr Genet 48:109-116.

Reinke, J., and Berthold, G. 1879. Die Zersetzung der Kartoffel durch Pilze. Untersuchungen aus dem Botanischen Laboratorium der Universität Göttingen:1-100.

Robb, J., Moukhamedov, R., Hu, X., Platt, H., and Nazar, R.N. 1993. Putative subgroups of Verticillium albo-atrum distinguishable by PCR-based assays. Physiol. Mol. Plant Pathol. 43.

Robinson, N., Platt, H.W., and Hale, L.R. 2007. Verticillium dahliae interactions with $V$. albo-atrum 'Group 2' and V. tricorpus and their effects on Verticillium wilt disease development in potato. Am. J. Potato Res. 84:229-235.

Rowe, R.C., and Powelson, M.L. 2002. Potato early dying: management challenges in a changing production environment. Plant Dis 86:1184-1193.

Rozen, S., and Skaletsky, H. 2000. Primer3 on the WWW for general users and for biologist programmers. Methods Mol Biol 132:365-386.

Sachs, M.S. 1996. General and cross-pathway controls of amino acid biosynthesis. . In: The Mycota: Biochemistry and Molecular Biology, ed. R. Brambl and G.A., Marzluf Heidelberg, Germany: Springer Verlag III:315-345.

Saitou, N., and Nei, M. 1987. The neighbor-joining method: a new method for reconstructing phylogenetic trees. Mol Biol Evol 4:406-425.

Sakuta, C., Oda, A., Yamakawa, S., and Satoh, S. 1998. Root-specific expression of genes for novel glycine-rich proteins cloned by use of an antiserum against xylem sap proteins of cucumber. Plant Cell Physiol 39:1330-1336.

Sanger, F., and Coulson, A.R. 1975. A rapid method for determining sequences in DNA by primed synthesis with DNA polymerase. J Mol Biol 94:441-448.

Sasse, C., Bignell, E.M., Hasenberg, M., Haynes, K., Gunzer, M., Braus, G.H., and Krappmann, S. 2008. Basal expression of the Aspergillus fumigatus transcriptional activator CpcA is sufficient to support pulmonary aspergillosis. Fungal Genet Biol 45:693-704.

Schnathorst, W.C. 1981. Life cycle and epidemiology of Verticillium. In M. E. Mace, A. A. Bell, \& C. H. Beckmann (Eds.), Fungal wilt diseases of plants New York: Academic Press:81-111.

Schönig, B., Vogel, S., and Tudzynski, B. 2009. Cpc1 mediates cross-pathway control independently of Mbf1 in Fusarium fujikuroi. Fungal Genet Biol 46:898-908.

Schoustra, S.E., Debets, A.J., Slakhorst, M., and Hoekstra, R.F. 2007. Mitotic recombination accelerates adaptation in the fungus Aspergillus nidulans. PLoS Genet 3:e68. 
Schürch, A., Miozarri, J., and Hütter, R. 1974. Regulation of Tryptophan Biosynthesis in Saccharomyces cerevisiae: Mode of Action of 5-Methyl-Tryptophan and 5-MethylTryptophan-Sensitive Mutants J. Bacteriol. 117:1131-1140.

Sewell, G.W.F., and Wilson, J.F. 1964. Occurrence + dispersal of Verticillium conidia in xylem sap of hop (Humulus lupulus L.). Nature 204:901.

Shen, W.C., and Ebbole, D.J. 1997. Cross-Pathway and Pathway-Specific Control of Amino Acid Biosynthesis in Magnaporthe grisea. Fungal Genet Biol 21:40-49.

Shi, F.M., and Li, Y.Z. 2008. Verticillium dahliae toxins-induced nitric oxide production in Arabidopsis is major dependent on nitrate reductase. BMB Rep 41:79-85.

Singh, S., Braus-Stromeyer, S.A., Timpner, C., Valerius, O., von Tiedemann, A., Karlovsky, P., Druebert, C., Polle, A., and Braus, G.H. 2012. The plant host Brassica napus induces in the pathogen Verticillium longisporum the expression of functional catalase peroxidase which is required for the late phase of disease. Mol Plant Microbe Interact 25:569-581.

Singh, S., Braus-Stromeyer, S.A., Timpner, C., Tran, V.T., Lohaus, G., Reusche, M., Knufer, J., Teichmann, T., von Tiedemann, A., and Braus, G.H. 2010. Silencing of Vlaro2 for chorismate synthase revealed that the phytopathogen Verticillium longisporum induces the cross-pathway control in the xylem. Appl Microbiol Biotechnol 85:19611976.

Söchting, H.P., and Verreet, J.A. 2004. Effects of different cultivation systems (soil management, nitrogen fertilization) on the epidemics of fungal diseases in oilseed rape (Brassica napus L. var. napus). J Plant Diseases Protec. 111:1-29.

Stark, J.C. 1961. Das Aufreten der Verticillium: Tracheomykosen in Hamburger Gartenbaukulturen. Gartenbauwissenschaft 26:493-528.

Stergiopoulos, I., Kourmpetis, Y.A., Slot, J.C., Bakker, F.T., De Wit, P.J., and Rokas, A. 2012. In silico characterization and molecular evolutionary analysis of a novel superfamily of fungal effector proteins. Mol Biol Evol.

Steventon, L.A., Fahleson, J., Hu, Q., and Dixelius, C. 2002. Identification of the causal agent of Verticillium wilt of winter oilseed rape in Sweden, Verticillium longisporum. Mycol. Res. 106:570-578.

Subbarao, K.V., Chassot, A., Gordon, T.R., Hubbard, J.C., Bonello, P., Mullin, R., Okamoto, D., Davis, R.M., and Koike, S.T. 1995. Genetic relationships and cross pathogenicities of Verticillium dahliae isolates from cauliflower and other crops. Phytopathology 85:1105-1112.

Svenson, C.H., and Lerenius, C. 1987. An investigation on the effect of Verticillium wilt (Verticillium dahliae Kleb.) on oilseed rape. Working group integrated control in oilseed rape. IOBC/WPRS Bulletin X/4:30-34.

Sweeney, M.J., and Dobson, A.D. 1999. Molecular biology of mycotoxin biosynthesis. FEMS Microbiol Lett 175:149-163.

Taborda, C.P., da Silva, M.B., Nosanchuk, J.D., and Travassos, L.R. 2008. Melanin as a virulence factor of Paracoccidioides brasiliensis and other dimorphic pathogenic fungi: a minireview. Mycopathologia 165:331-339.

Tamura, K., Dudley, J., Nei, M., and Kumar, S. 2007. MEGA4: Molecular Evolutionary Genetics Analysis (MEGA) software version 4.0. Mol Biol Evol 24:1596-1599.

Thireos, G., Penn, M.D., and Greer, H. 1984. 5' untranslated sequences are required for the translational control of a yeast regulatory gene. Proc Natl Acad Sci U S A 81:50965100.

Thompson, J.D., Higgins, D.G., and Gibson, T.J. 1994. CLUSTAL W: improving the sensitivity of progressive multiple sequence alignment through sequence weighting, 
position-specific gap penalties and weight matrix choice. Nucleic Acids Res 22:46734680.

Tian, C., Kasuga, T., Sachs, M.S., and Glass, N.L. 2007. Transcriptional profiling of cross pathway control in Neurospora crassa and comparative analysis of the Gen 4 and CPC1 regulons. Eukaryot Cell 6:1018-1029.

Timpner, C. 2008. Identifizierung differentiell exprimierter Biomarker zur Untersuchung der spezifischen Pathogen/Wirt-Interaktion von Verticillium longisporum mit Brassica napus. Diploma thesis, Universität Göttingen.

Timpner, C., Braus-Stromeyer, S. A., Tran, V. T. and Braus, G.H. (2013) The Cpc1 regulator of the cross-pathway control of amino acid biosynthesis is required for pathogenicity of the vascular pathogen Verticillium longisporum. Mol Plant Microbe Interact doi.org/10.1094/MPMI-06-13-0181-R

Tournu, H., Tripathi, G., Bertram, G., Macaskill, S., Mavor, A., Walker, L., Odds, F.C., Gow, N.A., and Brown, A.J. 2005. Global role of the protein kinase Gen2 in the human pathogen Candida albicans. Eukaryot Cell 4:1687-1696.

Trail, F., Mahanti, N., and Linz, J. 1995. Molecular biology of aflatoxin biosynthesis. Microbiology 141 ( Pt 4):755-765.

Tran, V.T. 2011. Adhesion of the rapeseed pathogen Verticillium longisporum to its host Brassica napus. Dissertation, Universität Göttingen.

Tran, V.T., Braus-Stromeyer, S.A., Timpner, C., and Braus, G.H. 2013. Molecular diagnosis to discriminate pathogen and apathogen species of the hybrid Verticillium longisporum on the oilseed crop Brassica napus. Appl Microbiol Biotechnol 97:44674483.

Tripathi, G., Wiltshire, C., Macaskill, S., Tournu, H., Budge, S., and Brown, A.J. 2002. Gcn4 co-ordinates morphogenetic and metabolic responses to amino acid starvation in Candida albicans. EMBO J 21:5448-5456.

Tzima, A., Paplomatas, E.J., Rauyaree, P., and Kang, S. 2010. Roles of the catalytic subunit of cAMP-dependent protein kinase A in virulence and development of the soilborne plant pathogen Verticillium dahliae. Fungal Genet Biol 47:406-415.

Underwood, W. 2012. The plant cell wall: a dynamic barrier against pathogen invasion. Front Plant Sci 3:85.

Valerius, O., Kleinschmidt, M., Rachfall, N., Schulze, F., Lopez Marin, S., Hoppert, M., Streckfuss-Bomeke, K., Fischer, C., and Braus, G.H. 2007. The Saccharomyces homolog of mammalian RACK1, Cpc2/Asc1p, is required for FLO11-dependent adhesive growth and dimorphism. Mol Cell Proteomics 6:1968-1979.

Vining, L.C. 1990. Functions of secondary metabolites. Annu. Rev. Microbiol 44:395-427.

Voigt, C.A., Schafer, W., and Salomon, S. 2005. A secreted lipase of Fusarium graminearum is a virulence factor required for infection of cereals. Plant J 42:364-375.

Wang, J.Y., Cai, Y., Gou, J.Y., Mao, Y.B., Xu, Y.H., Jiang, W.H., and Chen, X.Y. 2004. VdNEP, an elicitor from Verticillium dahliae, induces cotton plant wilting. Appl Environ Microbiol 70:4989-4995.

Wanke, C., Eckert, S., Albrecht, G., van Hartingsveldt, W., Punt, P.J., van den Hondel, C.A., and Braus, G.H. 1997. The Aspergillus niger GCN4 homologue, cpcA, is transcriptionally regulated and encodes an unusual leucine zipper. Mol Microbiol 23:23-33.

Wilhelm, S. 1955. Longevity of the Verticillium wilt fungus in the laboratory and field. Phytopathology 45:180-182.

Xiao, C.L., and Subbarao, K.V. 2000. Effects of irrigation and Verticillium dahliae on cauliflower root and shoot growth dynamics. Phytopathology 90:995-1004. 
Yu, J.H., and Keller, N. 2005. Regulation of secondary metabolism in filamentous fungi. Annu Rev Phytopathol 43:437-458.

Zare, R., and Gams, W. 2008. A revision of the Verticillium fungicola species complex and its affinity with the genus Lecanicillium. Mycol Res 112:811-824.

Zare, R., Gams, W., Starink-Willemse, M., and Summerbell, R.C. 2007. Gibellulopsis, a suitable genus for Verticillium nigrescens, and Musicillium, a new genus for $V$. theobromae. Nova Hedwigia 85:463-489.

Zeise, K., and von Tiedemann, A. 2001. Morphological and physiological differentiation among vegetative compatibility groups of Verticillium dahliae in relation to $V$. longisporum. J Phytopathology 149:469-475.

Zeise, K., and von Tiedemann, A. 2002a. Host Specialization among Vegetative Compatibility Groups of Verticillium dahliae in relation to Verticillium longisporum. J Phytopathology 150:112-119.

Zeise, K., and von Tiedemann, A. 2002b. Application of RAPD-PCR for virulence type analysis within Verticillium dahliae and V. longisporum J. Phytopathology 150:557563.

Zhou, B.J., Jia, P.S., Gao, F., and Guo, H.S. 2012. Molecular characterization and functional analysis of a necrosis- and ethylene-inducing protein encoding gene family from Verticillium dahliae. Mol Plant Microbe Interact.

Zhou, L., Hu, Q., Johannson, A., and Dixelius, C. 2006. Verticillium longisporum and Verticillium dahliae: Infection and disease in Brassica napus. Plant Pathol 55:137144.

Zielinski, D., and Sadowski, C. 1995. A preliminary study on Verticillium dahliae Kleb. In winter oilseed rape in Poland. Presented at the 9th International GCIRC Rapeseed Congress, 4-7 July 1995, Cambridge, F28: Pests and Diseases:649-651.

Zinkernagel, V. 1982. Zur Entwicklung von Verticillium spp. in anfälligen und toleranten Hopfensorten nach natürlicher und künstlicher Infektion. Zeitschrift für Pflanzenkrankheiten und Pflanzenschutz 89:205-218. 


\title{
Lebenslauf
}

\section{Persönliche Daten}

\author{
Name: Christian Timpner \\ Geburtstag: 10.11.1979 \\ Geburtsort: Northeim, Niedersachsen \\ Staatsangehörigkeit: deutsch
}

\section{Wissenschaftlicher Bildungsweg}

9/2009 - 5/2013 wissenschaftlicher Mitarbeiter im Institut für Mikrobiologie und Genetik, Göttingen in der Abteilung Molekulare Mikrobiologie und Genetik bei Prof. Dr. Gerhard H. Braus zur Anfertigung der Dissertation mit dem Titel " Roles of the Cpc1 regulator of the cross-pathway control in the Verticillium plant pathogens"

5/2008 - 9/2009 wissenschaftlicher Mitarbeiter im Institut für Mikrobiologie und Genetik, Göttingen

2007 - 2008 Institut für Mikrobiologie und Genetik, Göttingen Abteilung: Molekulare Mikrobiologie und Genetik Diplomarbeit zu dem Thema „Identifizierung differentiell exprimierter Biomarker zur Untersuchung der spezifischen Pathogen/Wirt-Interaktion von Verticillium longisporum mit Brassica napus.

Abschluss: Diplom

2000 - 2007 Georg August-Universität Göttingen

Hauptstudium Diplom Biologie

Hauptfach: Mikrobiologie

Nebenfächer: Botanik

Immunologie

\section{Schulische Ausbildung}

Schulabschluss Allgemeine Hochschulreife

1992 - 1999 Gymnasium Corvianum, Northeim

1990 - 1992 Orientierungsstufe Gerhart Hauptmann Schule; Northeim

1986 - 1990 Grundschule Edesheim 
Hiermit erkläre ich an Eides Statt, dass die Dissertation mit dem Titel „, Roles of the Cpc1 regulator of the cross-pathway control in the Verticillium plant pathogens" selbständig und ohne unerlaubte Hilfe angefertigt wurde.

Göttingen, im September 2013

$$
\text { Christian Timpner }
$$

